\title{
Exploring exposure in vivo in chronic pain
}

Citation for published version (APA):

de Jong, J. R. (2010). Exploring exposure in vivo in chronic pain. [Doctoral Thesis, Maastricht University]. Datawyse / Universitaire Pers Maastricht. https://doi.org/10.26481/dis.20101125jj

Document status and date:

Published: 01/01/2010

DOI:

10.26481/dis.20101125jj

Document Version:

Publisher's PDF, also known as Version of record

\section{Please check the document version of this publication:}

- A submitted manuscript is the version of the article upon submission and before peer-review. There can be important differences between the submitted version and the official published version of record.

People interested in the research are advised to contact the author for the final version of the publication, or visit the DOI to the publisher's website.

- The final author version and the galley proof are versions of the publication after peer review.

- The final published version features the final layout of the paper including the volume, issue and page numbers.

Link to publication

\footnotetext{
General rights rights.

- You may freely distribute the URL identifying the publication in the public portal. please follow below link for the End User Agreement:

www.umlib.nl/taverne-license

Take down policy

If you believe that this document breaches copyright please contact us at:

repository@maastrichtuniversity.nl

providing details and we will investigate your claim.
}

Copyright and moral rights for the publications made accessible in the public portal are retained by the authors and/or other copyright owners and it is a condition of accessing publications that users recognise and abide by the legal requirements associated with these

- Users may download and print one copy of any publication from the public portal for the purpose of private study or research.

- You may not further distribute the material or use it for any profit-making activity or commercial gain

If the publication is distributed under the terms of Article $25 \mathrm{fa}$ of the Dutch Copyright Act, indicated by the "Taverne" license above, 


\section{EXPLORING EXPOSURE IN VIVO IN CHRONIC PAIN}




\section{(C) 2010 Jeroen R. de Jong, Maastricht}

Cover illustration: Marcel Paulussen

Production: Datawyse | Universitaire Pers Maastricht

\section{ISBN 9789052789989}

The Studies in this thesis are supported by grants from NWO Social Sciences Research Council of the Netherlands, Grant nr. 453-04-003.

Nederlandse Vereniging van Posttraumatische Dystrofie Patiënten.

Uitgeverij Boom. 


\title{
EXPLORING EXPOSURE IN VIVO IN CHRONIC PAIN
}

\author{
PROEFSCHRIFT \\ Ter verkrijging van de graad van doctor aan de Universiteit Maastricht, \\ op gezag van de Rector Magnificus, Prof. mr. G.P.M.F. Mols, \\ volgens het besluit van het College van Decanen, \\ in het openbaar te verdedigen \\ op donderdag 25 november 2010 om 16.00 uur \\ door \\ Jeroen Roelof de Jong \\ Geboren op 1 mei 1970 te Tilburg
}

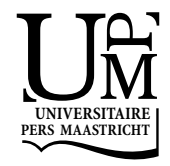




\section{Promotor}

Prof. dr. J.W.S. Vlaeyen

\section{Beoordelingscommissie}

Prof. dr. M.L. Peters (voorzitter)

Prof. dr. M. van Kleef

Prof. dr. S.J. Linton (Őrebro University, Őrebro, Sweden)

Prof. dr. R.J.E.M. Smeets

Dr. D. Vansteenwegen (KU Leuven, Leuven, België) 


\section{Table of contents}

$\begin{array}{lll}\text { Chapter } 1 & \text { General introduction } & 7\end{array}$

Chapter $2 \quad$ Pain-related fear, perceived harmfulness of activities and 41 functional limitations in Complex Regional Pain Syndrome type I

Chapter 3 Reduction of Pain-Related Fear and Disability in Post-

Traumatic Neck Pain: A Replicated Single-Case Experimental Study of Exposure in Vivo

Chapter 4 Reduction of pain-related fear in complex regional pain syndrome type I: The application of Graded Exposure in Vivo

Chapter 5 Fear of Movement/(Re)injury in Chronic Low Back Pain:

Education or Exposure In Vivo as Mediator to Fear

Reduction?

Chapter 6 Generalization of Graded Exposure in Vivo in Complex

Regional Pain Syndrome type I (CRPS-I)

Chapter 7 Summary and general discussion

Nederlandse samenvatting (Dutch summary)

References

Dankwoord (Words of thanks)

About the author

Publications 

Chapter 1

General introduction 


\section{Pain}

For many years pain was conceptualized, managed, and studied as a sensory experience and a symptom of pathology or injury. However, the conscious perception of pain does not always directly reflect incoming signals from primary sensory neurons (Melzack \& Wall, 1994; Petrovic \& Ingvar, 2002). At a higher level, cognitive process seems to modulate the perception of pain. As incoming sensory input becomes incorporated into the texture of cognition, it undergoes extensive associative elaboration and modulation (Mesulam, 1998). Pain is a multidimensional experience, which includes both sensory components (e.g. intensity, quality and spatiotemporal characteristics of the sensation), and affective-motivational components (i.e. the unpleasantness and aversiveness of the sensation) and which has no one-to-one relationship with the amount of tissue damage. For example, low back pain research has shown that the extent of disc herniation rarely relates to the pain experienced (e.g. Jensen et al, 1994). However, the ratio of the amount of injury to the amount of pain swings the other way too. For example, a paper cut creates a rather small incision, but hurts for most people attending to it. Other times, the pain system actually fails. Some life-threatening diseases, like cancer, are not painful, which is the reason they can affect the organism unnoticeably. Pain is not just a symptom; it can persist and become a patho-physiologic condition in itself. Functional disability and movement dysfunction are not just a consequence of anticipating and minimizing pain, but are one element of a much broader complex pain problem. There are many factors that are important in determining what hurts for whom and when, for example context, meaning, and the presence of competitive stimuli. One example to illustrate this: exactly the same minor finger injury will cause more pain in a professional violinist than in a professional ballerina because finger damage poses a greater treat to the violist. The event plays a greater role in the violinist's life goals and identity. There are different dimensions of a noxious stimulus context that may influence how it is experienced. Experimental studies showed that meaning (perceived tissue damaging properties) affects pain experience, a noxious stimulus evokes pain, and that warning and visual attention moderate the effects of meaning when the meaning is associated with tissue-damage (e.g. Arntz \& Claassens, 2004; Moseley \& Arntz, 2007). Different dimensions of the stimulus' context can have differential effects on sensory-discriminative and affective-emotional components of pain.

\section{Neurocognitive aspects of pain}

A fundamental principle of the complexity is that pain is produced by the brain when it perceives that danger to body tissue exists and that action is required (Merskey \& Bogduk, 1994). Imaging studies demonstrate that there is no single 'pain 
centre'. Many cortical areas can be activated during pain and wide variability exists within and between individuals (e.g. Ingvar, 1999). However, some cortical areas are involved more often than others. Most studies identify a network of primary and secondary somatosensory, insular, anterior cingulate, and prefrontal cortices and thalamus receiving parallel inputs from multiple nociceptive pathways (e.g. Ingvar, 1999; Creac'h et al., 2000; Apkarian et al., 2001; Bantick et al., 2002). In contrast to pain in normal subjects, decreased incidence of activity across these brain areas has been observed in patients (e.g. Apkarian et al., 2005). The anterior cingulated cortex (ACC) can be considered as the action centre (Craig, 2002). It is considered to serve to establish an emotional valence of pain, and coordinate the selection and planning of an appropriate behavioral response strategy (Price, 2000). Similar ACC activity has been reported during non-nociceptive but biologically threatening events such as anticipated pain (Sawamoto et al., 2000) and anxiety (Osuch et al., 2000) and the ACC is chronically active in chronic pain patients (Hsieh et al., 1995). Further, in chronic pain conditions, including cancer pain, headache, visceral pain and neuropathic pain, there seems a preferential activation of the prefrontal cortex (PFC) (e.g. Apkarian et al., 2005). The PFC has different subdivisions that are thought to play specific roles in various cognitive, emotional and memory functions. The preferential activation of then PFC underscores the conjecture that chronic pain states have stronger cognitive, emotional and introspective components than acute pain conditions, and that chronic pain conditions may be a reflection of decreased sensory processing and enhanced emotional and cognitive processing.

Another critical and as well complex component is that pain is experienced in the body image that is held by the brain, the so-called 'virtual body' in the primary somatosensory cortex (e.g. Melzack et al., 1997). This part of the brain is organized in 'maps' that represent a point-to-point connection between well-defined parts of the body and their respective anatomical location in the cortex. It is found that these maps change in response to (behavioral) experience and injury (e.g. Flor, 2002). Research has shown that chronic pain leads to an expansion of the cortical representation zone related to nociceptive input similar to the expansions observed with other types of behaviorally relevant stimulation (e.g. Flor, 2002). Katz and Melzack (1990) suggested that somatosensory memories play an important role in establishing cortex reorganization and chronic pain. According to their hypothesis, this means that experience of pain leads to a memory trace in the brain. In addition, it should be emphasized that this is a type of implicit memory that leads to behavioral and perceptual changes, such as hyperalgesia and allodynia, of which the patient is not aware. If pain experience is stored in memory, there is a possibility that the patient experiences pain even after the disease has been cured or the injury has healed. Research has shown that somatosensory pain memories manifest themselves in alterations in the S1 cortex, which means that they may contribute to hy- 
persensitivity even in the absence of peripheral stimulation (e.g. Flor, 2002). In addition, memories can be further established or enhanced by psychological processes, such as conditioning or attention (e.g. Flor, 2002). Furthermore, chronic states of pain are also associated with increased cortical excitation that may significantly contribute to reorganization.

\section{Summary}

In sum, pain experiences are normal responses to perceived threat to the integrity of the body. However, the level of pain does not necessarily reflect the amount of body tissue damage. The construction of the pain experience relies on many sensory and affective cues which are involved simultaneously. Although consistent patterns can be seen during pain experiences, the exact parts and amount of activity vary between people and even between several occasions in the same person (e.g. Peyron et al., 2000). Moreover, when pain persists and becomes chronic, the affective response system seems to be more involved and may increasingly contribute to the pain problem, while sensory processing may play a lesser role to pain experience. For example, pain-related fear or the anticipation of pain may be enough to predispose to pain experience if maintained long term (e.g. Vlaeyen \& Linton, 2000).

\section{Pain-related fear}

Fear is described as a present-oriented state that is designed to protect the individual from a perceived immediate state (e.g. Izard, 1998). It is the emotional manifestation of the fight or flight response (Cannon, 1992). In contrast to fear, anxiety is a seen as a future-oriented cognitive-affective state which occurs in response to anticipated threats that are often vague or uncertain in nature (e.g. Barlow, 2002). Research studies focusing on the explanation of chronic pain and its associated disability showed that pain-related fear plays an important role in the domain of pain (e.g. Vlaeyen \& Linton, 2000; Leeuw et al., 2007a). Pain-related fear can best be defined as the fear that emerges when stimuli that are related to the pain problem are perceived as a main threat. Pain-related fear is not a unitary construct and probably encompasses several forms of fear. For example, pain patients may fear the causes and consequences they assume to be associated with their pain. Depending on the current concerns, different stimuli are attended to and possibly misinterpreted. Patients suffering pain report fear of 'interoceptive stimuli' (e.g. mild pain, physiological sensations like feeling a 'crack', sudomotor activity, changes in skin blood flow) which often are interpreted as sign of (re)injury, 'proprioceptive stimuli' (e.g. activities, movements) which are perceived as harmful and may cause (re)injury, and 'exteroceptive stimuli' (e.g. doctors information, CT-scan, other pa- 
tients who now sitting in a wheelchair) which are related to patients fear to be or become (e.g. work-loss, wheelchair-bounded).

Kori et al. (1990) introduced the term 'kinesiophobia' (kinesis = movement) for the condition in which a patient has 'an excessive, irrational, and debilitating fear of physical movement and activity resulting from a feeling of vulnerability to painful injury or reinjury'. Research revealed that during confrontation with feared movements, pain patients who reported pain-related fear typically show a fear response comprising of psycho-physiological (e.g. muscle reactivity), behavioral (e.g. escape and avoidance behavior) as well as cognitive (e.g. think in catastrophes) elements. This supports the idea that chronic pain and chronic fear share important characteristics (e.g. Vlaeyen \& Linton, 2000). Indeed, when comparing the major features of specific phobia according to the criteria of the Diagnostic and Statistical Manual of Mental Disorders $4^{\text {th }}$ edition (DSM-IV: American Psychiatric Association, 1994) and pain-related fear in chronic pain patients, there is much similarity between the two conditions (table 1) (Kori et al., 1990). One point on which the two conditions differ is that people with a phobia are aware that the fear is excessive and irrational, whereas most pain patients reporting pain-related fear are convinced of the fact that avoidance has a protective function.

Table 1 Differences and similarities between specific phobia (according to DSM-IV) and pain-related fear (Kori et al., 1990)

Specific phobia Pain-related fear

1. Marked and persistent fear that is excessive 1. Marked and persistent fear that is (often) excessive or unreasonable cued by the presence or an- and unreasonable, cued by the presence or anticiticipation of a specific object or situation. pation of a pain-eliciting situation.

2. Exposure to the phobic stimulus almost 2. Exposure to the pain-eliciting stimulus almost invariably provokes an immediate anxiety response, which may take the form of a situinvariable provokes an immediate anxiety response, including avoidance/escape/safety-seeking behavational predisposed panic attack. iors, increased arousal levels, and hypervigilance.

3. The person recognizes that the fear is exces- 3 . The person often does not recognize that the fear is sive or unreasonable. excessive or unreasonable.

4. The phobic situation is avoided or else is 4 . The phobic situation is avoided or else is endured endured with intense anxiety or distress. with intense anxiety or distress.

5. The avoidance, anxious anticipation, or 5. The avoidance, anxious anticipation, or distress in distress in the feared situation(s) interferes significantly with the person's normal routine, occupational (or academic) functioning, or social activities or relationships, or there is marked distress about having the phobia. the feared situation(s) interferes significantly with person's normal routine, occupational (or academic) functioning, or social activities or relationships, ore there is marked distress about having the pain problem.

6. In individuals under age 18 years, the dura- 6 . Not considered relevant. tion is at least 6 months.

7. The anxiety, panic attacks, or phobic avoid- 7. The anxiety, panic attacks, or phobic avoidance ance associated with the specific object or situation are not better accounted for by another mental disorder. associated with the specific object or situation are not better accounted for by another mental or physical disorder. 


\section{Safety-seeking behaviors}

It has repeatedly shown that pain-related fear is associated with safety-seeking behaviors. Recently, Tang et al. (2007) identified in chronic back pain patients with high health anxiety a wide range of safety-seeking-behaviors (SSBs). SSBs are defined as behaviors performed as a strategy intended to prevent or minimize a feared catastrophe (e.g. Salkovskis \& Bass, 1997). SSBs refers not only to total avoidance of a feared situation (e.g. "I don't lift anything heavy because that would damage my back") but also to escape behaviors which are performed to terminate exposure to a feared situation (e.g. "I will stop garden at the first sign of pain in case I damage my back") and subtle avoidance behaviors which are performed to prevent the feared catastrophe while remaining in the feared situation (e.g. "I take care that I don't lean forward when I'm holding something heavy because that might break my spine"). Health anxiety is different from pain-related fear in that individuals with health anxiety are afraid to compromise their health, whereas pain-related fear is focused on pain and injury, it seems, given the similarity of both concepts, that SSBs may have relevance for pain-related fear. In the study of Tang et al. the SSBs had the common goal of protecting from further pain, injury or danger. Some of the SSBs were similar to an overt pain behavior (e.g. 'holding the back'), while some others were very subtle (e.g. 'continually testing the stomach muscles') and creative (e.g. 'constantly rocking, shifting the weight between the legs'). Most SSBs continued throughout the time the patient remained in a feared situation. The authors suggested that the use of SSBs could have two unintended effects: firstly, to exacerbate pain (e.g. "using the strength of the arm instead of the strength of my body") and, secondly, to prevent the disconfirmation of catastrophizing beliefs (e.g. "something drastic will happen unless I do this"), thus potentially maintaining the anxiety and disability in the long term, and maintaining pain itself. Furthermore, by using physical performance tests some studies even reported that behavioral performance appeared to be more strongly associated with pain-related fear than with pain severity (Crombez et al., 1999; Vlaeyen et al., 1995). In addition, Waddell et al. (1993) demonstrated that fear-avoidance beliefs about work are strongly related to overall functional disability, and more so than pain, and concluded that 'fear of pain and what we do about it may be more disabling than pain itself'.

In line with the cognitive theory of anxiety, a number of studies have also shown that pain-related fear is associated with heightened vigilance to pain and noxious body stimuli and increased body awareness (Crombez et al., 2004; Goubert et al., 2004; Roelofs et al., 2003). Hyper-vigilance refers to an increased attention and scanning of the environment for a feared stimulus and it emerges when pain is appraised as threatening and a patient's current goal is to escape or to avoid pain (Crombez et al., 2005). In such cases, hyper-vigilance to pain is driven by top-down instead of bottom-up variables. Because hyper-vigilance is largely the result of au- 
tomatic processes, this may undermine the efficacy of attentional coping strategies, in particular distraction (Crombez et al., 2005). For precise conclusions about which processes underlie hypervigilance, specific components of attention have been proposed (e.g. Posner \& Petersen, 1990): 1) initial orienting of attention (shift component), 2) focusing of attention (engagement component), and 3) directing attention away (disengagement component). Van Damme et al. (2006) showed that hypervigilance to pain signals does not emerge as rapid initial shifting to the pain signal but rather as enhanced processing once it is detected.

\section{Fear-avoidance model of pain}

The fear-avoidance model of chronic musculoskeletal pain (e.g. Lethem et al., 1983; Vlaeyen et al., 1995; Asmundson et al 1999, Vlaeyen \& Linton, 2000; Leeuw et al., 2007a) assigns a prominent role to catastrophic (mis)interpretation of pain and the associated pain-related fear in the development and maintenance of chronic pain. This model is primarily developed as an explanation of why chronic low back pain problems and associated disability develop in a minority of patients who experience acute low back pain. Pain-related fear has shown to be associated with increased disability levels, not just in back pain but also in cervical pain (e.g. Turk et al., 2008), Complex Regional Pain Syndrome (formerly Reflex Sympathetic Dystrophy: Rose et al., 1992), non-traumatic arm pain (Feleus et al., 2007), burn pain (Sgroi et al., 2005), Post-Herpetic neuralgia (Rose et al., 1992), chronic headache (e.g. Nash et al., 2006), fibromyalgia (e.g. Turk et al., 2004), knee injury (Kvist et al., 2005) and osteoarthritis (Heuts et al., 2004). So, it seems that the fear-avoidance model may be useful as an heuristic framework for the explanation of why some individuals develop a chronic pain condition, independent of the medical diagnosis.

The basic tenet of the fear-avoidance model is the way in which pain is interpreted. Acute pain patients who perceive their pain as non-threatening, and are likely to maintain engagement in daily activities promote functional recovery. In contrast, when pain is catastrophically (mis)interpreted this gives rise to pain-related fear, and associated safety seeking behaviors such as avoidance and/or escape and hyper-vigilance, that can be adaptive in the acute pain stage, but paradoxically worsen the problem in the case of long-lasting pain. Specifically, continuous avoidance of activities and/or certain movements may result in disability (problems executing daily life tasks and activities), altered central processing (preventing or reversing some of the more subtle, higher central changes associated with the pain syndrome), neglect (Galer \& Jensen, 1999; Moseley et al., 2004), motor abnormalities (e.g. weakness, muscle spasm, lack of coordination, dystonia: Galer \& Jensen, 1999; Jänig \& Baron, 2004) and depressed mood, which in turn may perpetuate the pain problem. 
Although changes in cognitive factors (fear-avoidance beliefs, catastrophizing) are not always found to be significantly associated with changes in pain intensity (Woby et al., 2004), their relationship with disability has been repeatedly shown. Crombez et al. (2002) showed that patients scoring high on pain-related fear over-predict the intensity of pain they will experience during physical examinations. In addition, compared to patients who report low levels of pain-related fear, fearful patients perform poorly on physical tasks (Vlaeyen et al., 1995). For low back pain patients, pain-related fear is a risk factor for the development of chronic low back pain through diminished participation in activities of daily life, greater perceived disability, greater work loss, and more frequent sick leave (Leeuw, Goossens, Linton, et al., 2007a), as well as poorer treatment performance (Asmundson, Norton, \& Norton, 1999). Furthermore, pain-related fear is a maintaining factor of pain-related disability due to its associations with escape/avoidance behavior already during the acute pain phase. Several prospective studies have demonstrated that fear avoidance beliefs influence the transition to chronic low back pain and associated outcomes, such as disability and sick leave (Waddell, 2004).

Most evidence concerning the validity of the fear avoidance model stems from cross-sectional studies, making it impossible to determine whether pain catastrophizing leads to poorer adjustment or vice versa (Keefe et al., 2004). However, using a cross-lagged correlational panel design, Burns et al. (2003) showed that earlytreatment reductions in pain catastrophizing and pain-related anxiety predicted late-treatment changes in pain severity, disability, and distress. Further, by the use of structural equation modelling, Goubert et al. (2004) showed that pain catastrophizing led to increased fear of movement, consequently leading to increased vigilance to pain and, finally, increased pain severity. In addition, using the same model, Cook et al. (2006) replicated the mediating role of pain-related fear between catastrophizing and pain severity. Further, Leeuw et al. (2007a) performed a large-scale population-based prospective study. Within a sample of low back pain patients these authors were not able to show that pain-related fear mediated the relationship between pain catastrophizing and functional disability as pain catastrophizing and disability were not related. However, pain catastrophizing was related to a measure of pain-related fear that patients completed six months later. In turn, painrelated fear predicted pain intensity and disability. Finally, Nieto et al. (2008) tested in sub-acute whiplash patients whether fear of movement is a mediator in the relation between catastrophizing and pain-related disability/depression as has been suggested by the fear-avoidance model. After controlling for descriptive variables and pain characteristics, pain catastrophizing and fear of movement were found to be predictors of disability and depression. Pain intensity was a predictor of disability but not of depression. The mediation effect of fear of movement in the relation- 
ships between catastrophizing and disability, and between catastrophizing and depression was also supported. The authors concluded that the results of this study are in accordance with the fear-avoidance model, and support a biopsychosocial perspective for whiplash disorders.

\section{Fear learning: Classical conditioning}

Classical or Pavlovian conditioning has traditionally been considered as model for the acquisition of pathological states of fear and anxiety. Anxiety disorders are considered having their origins in the acquired linking of an originally neutral stimulus with a biological relevant and threatening stimulus or situation. Unfortunately, there has been little research into the role of classical conditioning in the development of pain-related fear. However, it is likely that during an acute pain episode severe pain or another physical sensations as a sign of injury (unconditioned stimulus: US) and a harmless activity or movement (conditioned stimulus: CS) are causally associated. The result of this learning process is propositional knowledge about the relationship between the two stimuli (the movement with the increased pain as sign for injury) is stored in memory. In later phases, when the subject is exposed to the same kind of movement, the movement will activate the association with the increased pain, resulting in the elicitation of a conditioned response (CR). Even more than evoking a memory, this process results in a movement activating the active expectation of a threatening event. Because of its repeating (contingent) occurrence, the movement has also become a good predictor of the increased pain as sign for injury: 'When I lift up my four-year-old child, increase pain will follow which is a sign of irreparable damage of the intervertebral discs' and 'When I don't lift up my four-year-old child, pain will not increase, which means that the intervertebral discs will not be frayed'. The subject not only links two events (associative learning) but also learns that one event predicts another (expectancy learning). This expectancy learning will lead to the generalization of fear towards activities, movements and/or situations somewhat similar to the harmless movement that provoked and/or increased the pain. This new and threatening meaning of the originally neutral movement will change the subject's behavior. More and more activities, movements and/or situations are avoided.

Pain-related fear can develop in at least three different ways: direct experiences (e.g., a specific movement increases the pain or the swelling of the limb used); verbal information (e.g., a doctor indicates that arthrosis or an injury is involved); or observing others (e.g., a friend sitting in a wheel chair because of the same pain complaints or being unable to work). The way in which this association has come about is not important, since the outcome is always the same: an originally neutral stimulus provokes a pain-related fear because this stimulus was earlier causally 
associated with an aversive stimulus. Although fear conditioning is generally an adaptive and self-preserving form of learning, such conditioning may become a source of pathology when prolonged protective responses are elicited by an extending number of CSs in the absence of a US.

\section{The extinction of pain-related fear}

Based on the aforementioned findings one may conclude that pain-related fear could be an important target for intervention in pain syndromes. It is not clear whether fearful patients can benefit optimally from traditional health care. For example, regardless of the treatment message, fearful patients may sustain their avoidance behavior when the disadvantageous consequences of avoidance behavior are neither explicitly addressed, nor managed. Especially in traditional health care, in which pain is approached from a more biomedical orientation, the message that activity is beneficial is often accompanied by a note of caution, certainly when the pain increases. Consequently, it is not surprising if fearful patients may respond with more avoidance and safety behaviors to these biomedical treatments. One of the questions is how these treatments have been delivered, and whether pain-related fear and the associated safety behaviors were adequately addressed (Leeuw et al., 2007a).

In line with the idea of matching treatment to specific treatment characteristics (Vlaeyen \& Morley, 2005) several cognitive behavioral programs, comprising various treatment techniques directed at changing cognitions and improving behavioral participation in activities, have currently been developed (see Leeuw et al., 2007a). The results of these treatments suggest that such programs, and even brief educational sessions, can effectively decrease functional disability, which might be due to reducing fear-avoidance beliefs and catastrophically thoughts about pain-related stimuli. Pain-related fear might therefore be an essential target for successful interventions, and it can be suggested that methodically addressing and diminishing pain-related fear might even been more powerful. An example of a treatment that is designed for those reporting high levels of fear is exposure. Therefore, it may be the most suitable treatment to achieve the most significant reduction of painrelated fear.

\section{Summary}

In sum, there is accumulating support for the role of pain-related fear as explanation of why patients experience acute pain develops chronic pain problems and associated disability. Pain-related fear is associated with catastrophic (mis)interpretations of pain, hyper-vigilance, increased escape and avoidance behaviors, as well as intensified pain experience and functional disability, central 
change, motor abnormalities and depression. Exposure seems to be a suitable treatment.

\section{Exposure therapy}

One of the most effective strategies for treating anxiety disorders undoubtedly is exposure therapy with or without cognitive strategies and pharmacotherapy (e.g. Barlow, 2002). Exposure therapy is rooted in behaviorism and learning theories. In particular, Mowrer (1939) was one of the first who hypothesized that fears are acquired through repeated presentations of a neutral stimulus (conditioned stimulus: CS) and a pain-producing or fear-eliciting stimulus (unconditioned stimulus: US). In a series of rat studies he observed that the strength of the fear response is determined by the number of repetitions of association between the CS and US, and the intensity of the unconditioned response. Further, Mowrer (1939) also gave the initial impetus to exposure therapy in humans. He noted that the repeated presentation of the CS in the absence of the US leads to extinction, the gradual decrease of the conditioned response. Currently, exposure is seen as the process in which the patient is repeatedly exposed for prolonged periods to a feared object or situation in the company of a supported therapist and in the absence of aversive consequences; also named extinction training (e.g. Bouton et al., 2001; Davis et al., 2006; Quirk, 2006). Extinction is a well-known and important behavioral process that allows the organism to adapt its behavior to a changing environment. Animal research suggests that extinction is a form of acquired inhibition that suppresses a fear response and that extinguished conditioned responses can be recovered (e.g. Bouton, 2002; 2004; Rescorla, 2001). Examples of such conditions are the mere passage of time (spontaneous recovery) (e.g. Rescorla, 2004), context change after extinction (renewal) (e.g. Bouton \& Ricker, 1994), or the presentation of postextinction USonly trials (reinstatement) (e.g. Rescorla \& Herth, 1975). These phenomena support the view that extinction is not simply unlearning or forgetting but rather a new form of learning that changes the CS-US contingency in such a way that the CS no longer signals an aversive event and thereby inhibits the expression of the fear response (e.g. Bouton, 1993; 2004; Myers \& Davis, 2002; Rescorla, 1996; 2001). Given the often highly specialized functions of specific brain regions and the cognitive system of humans that is capable of a symbolic and propositional analysis of conditioning experiences (see De Houwer et al., 2005), there are no a priori reasons to assume that similar processes are not involved in extinction in humans.

Knowledge about the conditions that facilitate or hamper extinction learning may help to sharpen exposure treatments in such a way that treatment outcome can be maximized in the short run (therapy efficacy) as well as in the long run (relapse 
prevention). Hence, before describing graded exposure in vivo as therapy for painrelated fear, the most important findings from human fear extinction are reviewed briefly. The focus will be on the extinction principles to exposure therapy protocols in clinical groups with established fears.

\section{Extinction procedures in human phobic samples}

With regard to animal studies and laboratory studies in humans the experimental investigation of extinction procedures in human phobic samples is immediately disadvantaged by starting from the stage of fear reduction rather than fear acquisition. This means that the circumstances of acquisition are often unknown and there is usually no opportunity to test the original context. Nevertheless, experimental clinical studies are of great importance because of their potential relevance for enhancing treatments for anxiety disorders. Moreover, replicable effects have been observed.

\section{Safety signals and protection from extinction}

Consistent with the idea that avoidance responses may share some functional properties with Pavlovian safety signals (see Mineka \& Henderson, 1985), Salkovskis et al. (1991) have provided evidence that 'within-situation safety behaviors', equivalent to avoidance responses, interfere with the benefits of exposure therapy. Specifically, these authors showed that teaching anxious clients to refrain from these behaviors leads to greater fear reduction after an exposure session. They argued that clients attribute the absence of harm to their safety behaviors, rather than reducing their appraisal of the threatening stimulus. An alternative interpretation of these results derives from a protection from extinction perspective. Exposure to a phobic stimulus while using safety signals (avoidance responses or conditioned inhibitors) partially protects the phobic stimulus from extinction.

\section{Contextual effects on return of fear}

The majority of research has focused on context renewal effects, wherein contexts associated with successful exposure treatment are assumed to activate non-fearful memories and non-treatment contexts are assumed to elicit fearful memories and return of fear. Using graduated exposure therapy with live spiders by means of participant modeling in individuals who are highly fearful of spiders, context renewal effects have been observed when participants are retested 1 to 2 weeks later in a context that differs from the treatment context (e.g. Mineka et al., 1999). The effects became stronger with more distinctly different contexts and with various other improvements to methodology (Mystkowski et al., 2002). Context-based renewal also has been demonstrated via use of videotape exposure to a spider in a specific location of a house (Vansteenwegen et al., 2007). Not only did videotaped 
exposure lead to decreased electrodermal responding and disgust ratings compared with the control group (single context exposure), but both indices subsequently increased when tested with a videotape showing the same spider in a new context (e.g. a location in the house that was not previously seen) relative to the original exposure context.

Evidence for internal context specificity of extinction was demonstrated in a study of Mystkowski et al. (2003) in which drug state through caffeine versus placebo ingestion for individuals fearful of spiders was manipulated. Participants received one session of exposure therapy under the influence of a randomly chosen drug condition (placebo or caffeine) and were reassessed one week later after ingesting a drink mixture that was either the same or different than the drink ingested during the previous treatment session. The results showed that participants experiencing incongruent drug states exhibited significantly greater self-reported return of fear, measured during a behavioral approach task, from post-treatment to follow-up than those participants experiencing congruent drug states. Remarkable, the effects were comparable whether the shift was from caffeine to placebo or vice versa. However, the effect sizes were smaller than those achieved via the inside/outside context shifts.

An obvious implication of the above mentioned studies on the effects of contextual change on enhancing return of fear seems to be to apply exposure therapy in multiple contexts so that extinction memories can be cued by multiple contexts.

\section{Reinstatement effects}

Follow-up studies of exposure often report return of fear. Models of classical conditioning often focus on reinstatement as an explanation of relapse. Reinstatement is the return of avoidance, escape or safety-seeking behavior (conditioned responses) to a previously extinguished CS as a result of the confrontation with an unpredicted US-presentation. The US-only presentation reactivates the memorized CS-US association that is still present after successful exposure. Reinstatement studies in humans are rare and as far as known only documented in three experimental laboratory studies. The first two studies of Dirikx et al. (2004) and Hermans et al. (2005), in which a series of differential fear conditioning experiments were conducted, showed whenever CSs were tested after reinstatement there was a selective partial return of US-expectancy for the CS+ in a reinstatement group. In addition, selective reinstatement was observed in fear ratings as well. Van Damme et al. (2004), who used an emotional modification of a spatial cueing paradigm, demonstrated also successful reinstatement of attentional bias, resulting in facilitated engagement to threat signals, and in retarded disengagement from threat signals. Because one or a few unpredicted US experiences were capable of reinstalling conditioned responses, these authors assumed that this finding implicates that extinction does not result in simple unlearning of the association between a CS+ and a US. Further, the findings 
of this third study indicate that reinstatement is not limited to expectancy and arousal, but also extends to attentional processing. Finally, the study showed that attentional bias to threat can be extinguished by CS+ presentations without US reinforcement, suggesting that exposure may be an effective intervention to reduce hypervigilance as well. However, it should be mentioned that a simple reappearance of the object of threat may be sufficient to reinstate biased attentional processing of signals previously predicting threat. Contrary, clinical anecdotes about return of fear (Jacobs \& Nadel, 1985) and also theoretical accounts of classical condition (Wagner \& Brandon, 1989) indicate that an identical US-only experience is not needed to have reinstatement effects. Van Damme et al. (2004) suggested that it may be sufficient that the new US has similar affective attributes as the previous one.

A clinical example of reinstatement in relation to pain-related fear is when someone develops pain-related fear after experiencing a shooting pain in the lower back during his work. After a successful exposure treatment, this person has enough confidence to return to work. A few months after treatment, however, the person experiences a shooting pain in the lower back while playing with his/her children. This shooting pain could lead to a re-emergence of the expectancy of a shooting pain at work. The finding of reinstatement is intriguing, and has potential implications for pain-related fear. The example shows that exposure does not result in unlearning of the association between a US (pain) and a CS (activity), as one unpredicted shooting pain experience in the lower back is capable of reinstalling conditioned responses (e.g. avoiding work). It seems likely that pain-related fear can be easily reactivated. To clarify the specific mechanisms of and the situations in which reinstatement can occur, more research into pain-related fear is needed. For example, clinical experiences about return of pain-related fear, shows that an identical pain experience is not needed to have reinstatement effects. Van Damme et al. (2006) suggested that US experiences do not need to share sensory characteristics, but that it may be sufficient that they have the same affective attributes. Furthermore, it is also important that experimental and clinical research focuses on prevention of reinstatement in order to further increase the effectivity of exposure therapy.

\section{Cognitive processes in exposure therapy}

In cognitive therapy, dysfunctional beliefs about the dangerousness of the feared stimulus are assumed to play a crucial role in the maintenance and development of the fear response (e.g. Beck \& Emery, 1985; Clark, 1989; Salkovskis \& Clark, 1991; Hofmann, 2007). During treatment, the patient is provided with an opportunity to challenge these beliefs by rejecting hypotheses by exposing himself to situations that are likely associated with the expected harmful consequences. The fact that the 
patient is encouraged to re-evaluate harm expectancy appears to be the commonality between extinction learning and exposure therapy.

For a variety of chronic pain problems cognitive-behavioral therapies (CBT) have been demonstrated to be effective (e.g. Spinhoven et al., 2004; Smeets et al., 2006; Turner et al., 2007). In these studies changes in patient-related beliefs (e.g. catastrophic misinterpretation of increased pain) were shown to mediate the effects of CBT. Although cognitive-behavioral treatments share certain fundamental characteristics, they vary in goals and techniques (Jensen et al., 2001). Empirical evidence suggests that exposure procedures without explicit cognitive intervention strategies have very similar effects than comprehensive cognitive-behavioral treatments (e.g. Hofmann, 2004). Moreover, it has been shown that that treatment changes during exposure therapy are also mediated via changes in patient-related beliefs (Hofmann, 2004). These results are consistent with previous studies suggesting that simple exposure procedures lead to significant improvements in negative selfperception (Hofmann, 2000) and negative beliefs about social interactions (Newman et al., 1994).

By using exposure in vivo or behavioral experiments Arntz et al. (1994) investigated in anxiety patients the overprediction of anxiety phenomenon and its relationship with fear, dysfunctional beliefs, and emotional experiences during confrontations with feared stimuli. Independent of the executed treatment the study showed, clearly ad odds with the cognitive views, that expectations of anxiety have an additional influence on fear to that of dysfunctional beliefs. According to these authors it appears that the influence of change in dysfunctional beliefs on change in fear is exerted only via the change in anxiety prediction. This indicates that reduction of dysfunctional beliefs have an independent influence on reduction of fear. Further, Arntz et al. (1994) found strong evidence for the view that experienced unpleasantness of the (potentially) disconfirmatory experience negatively influences reduction of fear.

\section{Summary}

Exposure is seen as the process in which the patient is repeatedly exposed for prolonged periods to a feared object or situation and hence in the absence of aversive consequences; also named extinction. As a procedure, extinction refers to the repeated presentation of a CS in the absence of the US with which it was previously paired. Studies of fear extinction in human samples support the view that that extinction does not entail unlearning and that the addition of context changes and one or a few unpredicted US experiences after extinction can lead to a partial recovery of the apparently extinguished fear responses. The work on extinction in multiple 
contexts and use of reminder cues seems to be important in this respect. Furthermore, it appears that exposure therapy involves cognitive processes that influence dysfunctional beliefs.

\section{Graded exposure in vivo in chronic pain}

Because of many remarkable similarities between pain-related fear and fear in general, a graded exposure in vivo treatment protocol (GEXP) is developed for application in fearful pain patients. By the use of studies with replicated single-case experimental methodology Vlaeyen et al. (2001, 2002a, 2002b) were the first who illustrated that GEXP is a promising treatment in fearful chronic back pain patients. Hereafter, more single-case studies and randomized clinical trials have examined the effectiveness of exposure in vivo in (sub) acute and chronic low back pain patients. Besides effectiveness also generalization of exposure in chronic low back pain patients is investigated. Before reviewing the outcome studies on exposure in vivo for reducing pain-related fear and examining generalization, the clinical application of GEXP as developed by Vlaeyen and colleagues is described first.

\section{Clinical application}

The GEXP that was developed for patients with chronic pain who report substantial pain-related fear, and fear of movement/(re)injury in particular, is highly structured, protocolized, individually tailored, and aim to restore a normal pattern of daily function, including complete return to work (see Vlaeyen et al. 2002c, 2004). Due to gradual and repeated encounters with feared activities, the goal of GEXP is to provide patients with the most convincing evidence that expected detrimental consequences of these feared activities are in fact a catastrophic overestimation. Pain reduction and decrease of observed and reported physiological signs, such as edema, changes in skin blood flow or abnormal motor and sudomotor activity is not the primary goal. Usually, a therapist team consisting of a behavioral therapist and a paramedic (occupational therapist or physiotherapist) both experienced in the cognitive-behavioral rehabilitation of patients with chronic pain provides GEXP. Appropriate instruments to indicate the presence of pain-related fear are for example the Tampa Scale for Kinesiophobia (TSK: Miller et al., 1991) and the Photograph-series Of Daily Activities (PHODA: Dubbers et al., 2003; Jelinek et al., 2003; Kugler et al, 1999; Leeuw et al., 2007a). Following the suggestions of Philips (1987), who was the first suggesting to gradually expose pain patients to activities they feared and avoid during long time due to the belief that these cause damage and/or (re)injury, GEXP consists of several components: behavioral analysis, goal identification, education, graded hierarchies, exposure in vivo, and generalization. 


\section{Behavioral analysis}

The GEXP starts with an intake by the psychologist or behavioral therapist. The aim is to complete a behavioral, cognitive and psycho-physiological analysis of the pain problem with special attention to patient's catastrophic interpretations and to assess the role of pain-related fear in the context of the pain problem. It also includes information about the antecedents (situational or internal) of the pain-related fear and about the direct and indirect consequences. This screening might also include other areas of life stress, as they might increase arousal levels and indirectly also fuel pain-related fear. Phobic thoughts are often formed as conditional assumptions: 'If $P$, then $Q$ ' ( $P$ is the predictor and $Q$ is the consequence): "I' $m$ in pain so there must be something harmful happening to my body", Further, because patients do not see their complaints as a fear problem, the term harmfulness can better be used in the communication about there avoidance of physical activity. Sometimes, patients find it also hard to really estimate the harmfulness of a particular activity when they have avoided it extensively. In such cases, behavioral tests can be introduced. Finally, patients seem to search their conviction with regard to the sensitivity for (re)injury in the results of diagnostics tests. Therefore, it is preferable to have these tests available.

\section{Goal identification}

Besides the behavioral analysis, patients are also invited to formulate their own treatment goals. Chronic pain or frequent recurrent episodic pain may have any profound effects on a patient's life. The repeated interference with tasks that are essential to achieving various life goals and maintaining a patient's role in society will impact on their sense of self, both their current self and perhaps more importantly their plans and ideas about who they might become (Morley, 2008). Patients may experience a sense of 'suspended identity' in which they consider that the real them is suspended at an earlier time point, usually before the onset of chronicity (Risdon et al., 2003). One implication of this is that they use this point of time as a reference for setting goals of recovery (Morley, 2008). As a consequence of this the therapist makes clear that GEXP does not primarily aim at reducing pain but at the restoration of functional abilities through which patients' capacity to live according to their life values can be restored. Subsequently, the patient and therapist agree on one or more realistic and specific goals that are formulated in positive terms. Activities (e.g. lifting weights) that are in line with these goals (e.g. return to work) are those that will be included in GEXP.

\section{Education}

An educational session is provided by the therapist team explaining the treatment rationale. Patients are given a careful explanation of the fear-avoidance model (Vlaeyen et al., 1995) using their own individual symptoms, beliefs and behaviors in 
relation to their pain complaints. The therapist team illustrates the paradoxical and dysfunctional effects of avoidance as safety behavior, and offers the patient a new view on pain as a common condition that can be self-managed, rather than as a serious disease or a condition that needs careful protection. One of the major goals of the educational component is to help the patient understand that the consequences of pain are catastrophically overestimated.

\section{Graded hierarchies}

Direct after the explanation of the treatment rationale a hierarchy of fear-eliciting activities are made using the Photograph-series Of Daily Activities (PHODA; Leeuw et al. 2007b). This is a standardised method during which patients are requested to judge the harmfulness of diverse physical daily life activities represented by photographs. Using a (fear) thermometer, each picture is given a rating between zero (representing the situation which is not harmful for the upper extremity) to 100 (representing the situation which is absolutely damaging the upper extremity). Based on the graded hierarchy of the PHODA individually tailored practice tasks can be developed.

\section{Exposure in vivo}

Subsequently, during the next sessions patients are gradually and systematically exposed to the tailored and fear-provoking activities. During each exposure session patients are encouraged to engage in these fearful activities as much as possible until disconfirmation has occurred and anxiety levels have decreased. To demonstrate that the concerning activity is harmless and not extraordinary it is modelled first by one member of the therapist team. The exposure has the form of a series of behavioral experiments in which dysfunctional beliefs are explicitly being challenged. The essence of the behavioral experiment is that the patient performs an activity to challenge the validity of his catastrophic assumptions and misinterpretations. These assumptions take the form of "If . . . , then . . ." statements (e.g. "If I lift up my child, then pain increases which is a sign of nerve rupture and my muscles will get blocked") and are empirically tested during a behavioral experiment. Besides the dysfunctional proposition, a realistic alternative belief is formulated. Before and after each exposure trial, then credibility of both propositions is rated on a 0 to 100 numerical rating scale. For example, a back pain patient may expect that jumping down from a stair will inevitably cause nerve damage and results in an acute paralysis of the legs [credibility $=75 \%$ ]. Alternatively, the patient might expect that after jumping down, he or she will be able to continue walking without any problem [credibility $=25 \%$ ]. The behavioral experiment is designed and described as specific and concrete as possible. For example, if the patient is convinced that jumping down is harmful, the therapist team can further inquire about the minimal height that is needed to cause nerve injury. Before the experiment is carried out the 
therapist team asked the patient what he expects will happen during the experiment whenever the original and alternative belief is true. Afterwards the patient is requested to reflect on what he did learn from the experience. If subjective distress is significantly decreased, the patient and therapist team can consider moving on to the next item of the fear hierarchy. In order to facilitate independence and to promote more exposures, the therapists gradually withdraw their presence, as they may serve as initial safety signals.

\section{Effectiveness of graded exposure in vivo}

In the past years, the effectiveness of exposure in vivo has only been examined in studies with back pain patients. A summary of the outcome studies reviewed is presented in Table 2 and 3. First, studies using single-case designs will be discussed followed by a discussion of randomized clinical trials.

\section{Single-case studies}

In two studies of Vlaeyen et al. (2001, 2002a), a replicated single-case cross-over design was applied with four and six chronic back pain patients respectively. After a no-treatment baseline measurement period, the patients were randomly assigned to either GEXP first followed by an operant graded activity program (GA) or to a situation in which the treatment modules were reversed. GEXP as well as GA were imbedded in a rehabilitation program. Daily measures of pain-related cognitions, fears and disabilities were recorded with visual analogue scales. Before and after each treatment and at 6-month follow-up standardized measures of pain-related fear, pain catastrophizing, pain control and pain disability were taken. The results provided preliminary evidence showing that GEXP, compared with GA, was superior in decreasing pain-related cognitions and fears, pain control and pain disability. Not only were improvements found on self-report measures, but GEXP also generalized to increas of daily life activities as measured with ambulatory activity monitors. Although GEXP was provided during a period of 4 weeks, the reduction of painrelated fear was achieved within fewer than 3 exposure sessions of 1 hour. In these studies, the presentation of the rationale at the start of GEXP might have contributed to these abrupt changes. The third study of Vlaeyen et al. (2002b) revealed similar effects in two low back pain patients. In this study GEXP was delivered as the only treatment, and without a background rehabilitation program, which seems to suggest that it is GEXP that is responsible for the success. Without using ambulatory activity monitors Linton et al. (2002) replicated the results of Vlaeyen et al. (2001, $2002 a, 2002$ b) in a primary care facility of which two low back pain patients were recruited via a general practitioner. 


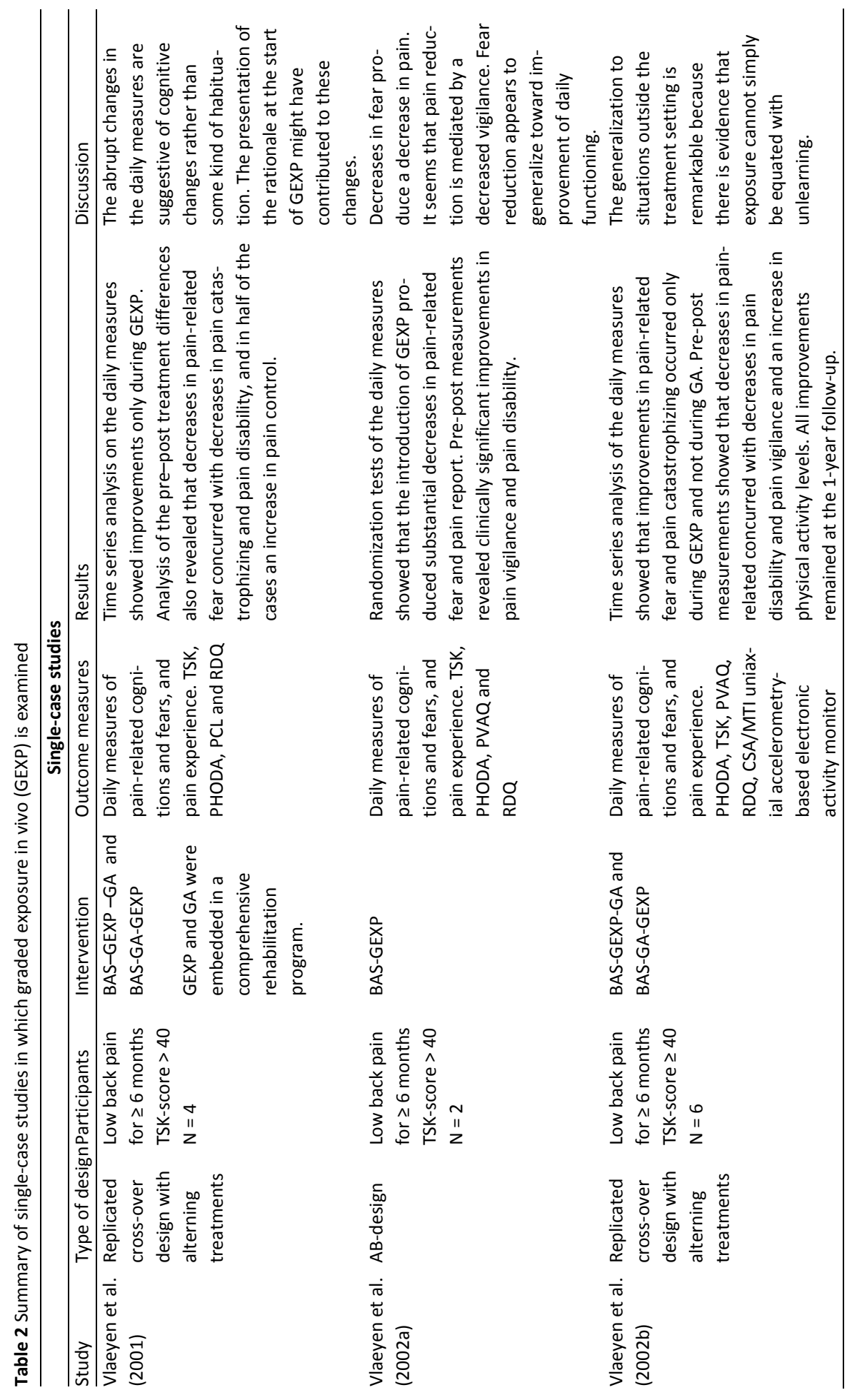




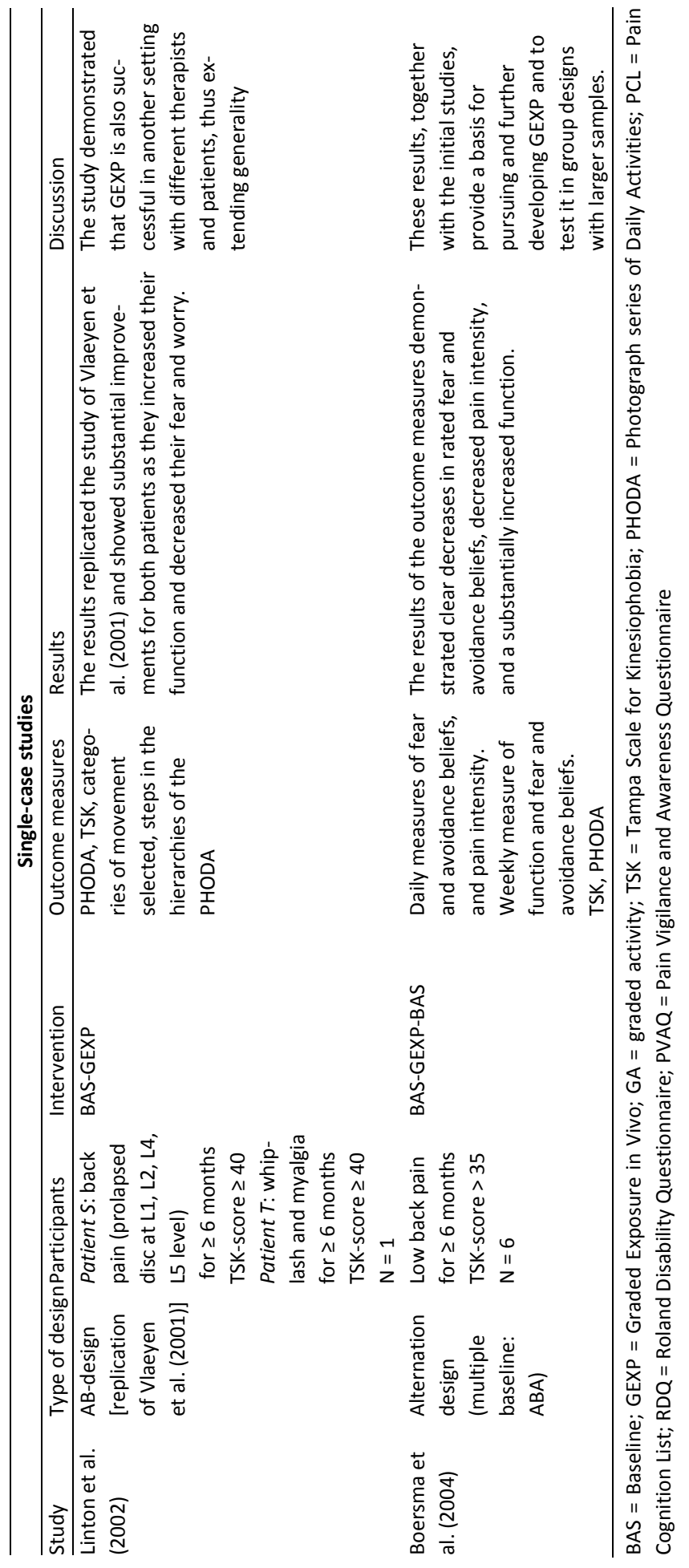


Finally, the external validity of the exposure treatment is also supported by a study of Boersma et al. (2004). A multiple baseline design was employed with six back pain patients who filled out daily and weekly measures during a 10-week period. The results demonstrated clear decreases in pain-rated fear and avoidance beliefs while function increased substantially. In contrast to the previous studies the improvements were not as large and changes did not occur as rapidly. The authors suggest that this could be due the relative inexperience of the current therapist team and the assessment methods used. Furthermore, there were no follow-up data by which the authors were unable to pronounce generalization effects.

\section{Randomized clinical trials}

Besides the above mentioned single-case studies also three randomized controlled trials (RCT) have corroborated the effectiveness of exposure in vivo for chronic low back pain patients. Linton et al. (2008) evaluated the effects of exposure in vivo in 46 patients who were randomized into an exposure plus usual treatment or waiting list control plus usual treatment (contact with a general practitioner, analgesics, and physical therapy). A particular feature of this study was that the exposure was aimed at fear of work-related activities. After the waiting period the control group crossed over and received the exposure treatment. The exposure group demonstrated a significantly larger improvement on function. Overall exposure had moderate effects on function, fear and pain intensity. However, according to the authors the results are quite valuable since they address generalization to a larger sample and since patients have been randomized thus reducing the obvious risk of patient selection in case studies.

Woods \& Asmundson (2008) randomized 46 patients to graded exposure in vivo, graded activity, or a wait-list condition. Despite the lack of a statistically significant improvement in daily functioning, patients who were randomized to the exposure group demonstrated also improvements in fear, both statistically and clinically, when compared to graded activity and the wait-list control condition. According to these authors the success of the exposure treatment can in particular be explained by the combination of the education component (illustration and explanation of the fear-avoidance model), the successful performance of graded exposure tasks, resumption of enjoyable activities, and increased comfort with and independence when performing tasks. This, in turn may have contributed to increasing the likelihood that treatment gains would be maintained.

Finally, Leeuw et al. (2008) examined the effectiveness as well as specific mediating mechanisms of GEXP versus operant graded activity (OGA) in 85 patients with chronic low back pain. Contrary to the study of Linton et al (2008) it was demonstrated that GEXP, despite excelling in diminishing pain catastrophizing and perceived harm- 
fulness of activities, was equally effective as OGA in improving functional disability and main complaints directly and six months after treatment, although the group difference almost reached statistical significance favouring GEXP. Although this study may suffer from a lack of statistical power, the authors discussed some other possible explanations for these findings. First, it was suggested that OGA and GEXP share components that may have strong therapeutic effects. Foremost, OGA may have included some form of exposure in vivo, since patients performed various activities, including those that were fear eliciting. In addition, OGA also included the explicit assurance that all activities were safe and allowed, as was communicated during the educational session, which may have resulted in modifications of patient pain-related beliefs. Finally, because therapists participated in both treatment conditions and therefore also were trained in GEXP, their attitude towards activities may have changed accordingly in the sense that they were less careful and worried about possible activity restrictions for their patients (Coudeyre et al., 2006). Second, OGA consisted of more treatment sessions than GEXP, and patients selected and performed activities that were of high importance during GA, whereas patients had to perform activities that were selected based on their threat value during GEXP. Furthermore, since the therapists were already accustomed to carrying out OGA, and had no experience with GEXP, it may be that specific treatment competence was superior in OGA. Fourth, outcome studies on psychotherapy showed that specific treatment ingredients only contribute marginally and that common treatment factors, such as therapeutic competence or patient's and therapist's belief, mainly explain the outcome (e.g. Wampold, 2000). The fact that in the study of Leeuw et al. (2008) GEXP and OGA may have shared more common features than that they comprised unique treatment ingredients might explain the borderline significant differences in reducing disability levels between GEXP and OGA. Despite decreased pain-related fear the patients in this study still reported pain after GEXP which also may contribute to the experience of discomfort in daily life. The latter is different from the single-case studies in which the daily measures showed that during GEXP a reduction in pain-related fear was followed by a decrease in pain experience.

\section{Generalization of exposure in vivo}

As already mentioned in greater detail, there is growing evidence that exposure cannot simply be equated with unlearning. Studies demonstrated that a competition occurs between the original threatening (excitatory) meaning of the stimuli and a new (inhibitory) meaning. In other words, during successful exposure, exceptions to the rule are learned rather than a fundamental change of that rule. Using behavioral tests several experimental studies investigated generalization of exposure to movements in chronic low back pain patients. Table 4 displays the most important characteristics and results of these studies. 


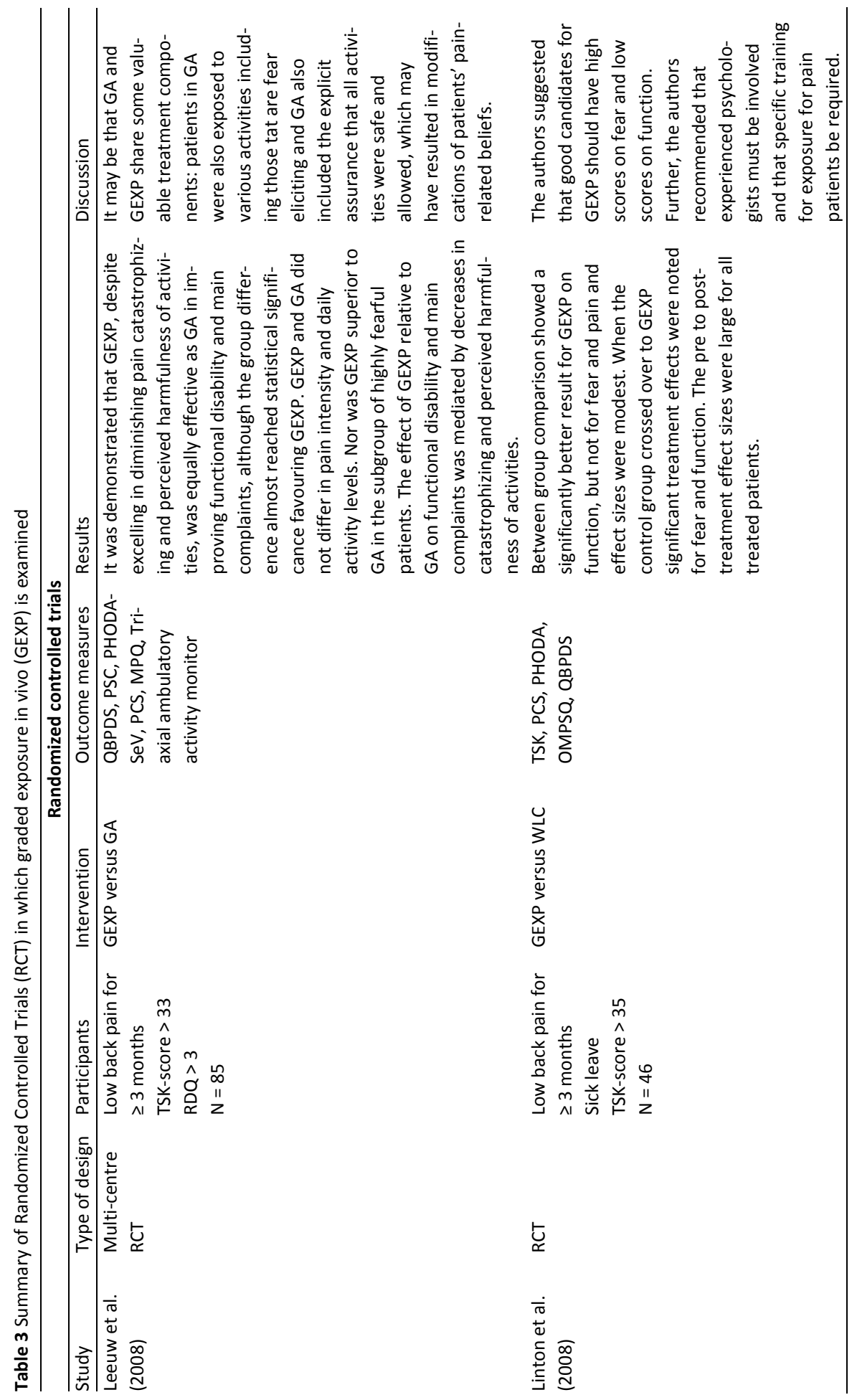




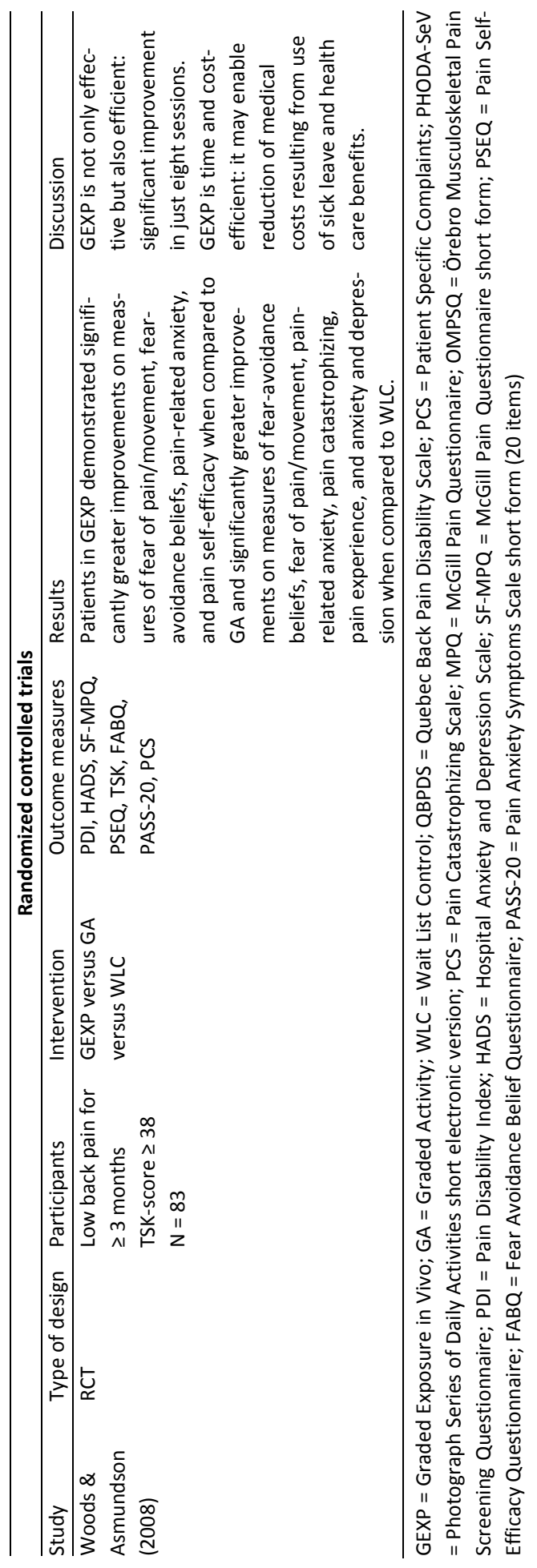




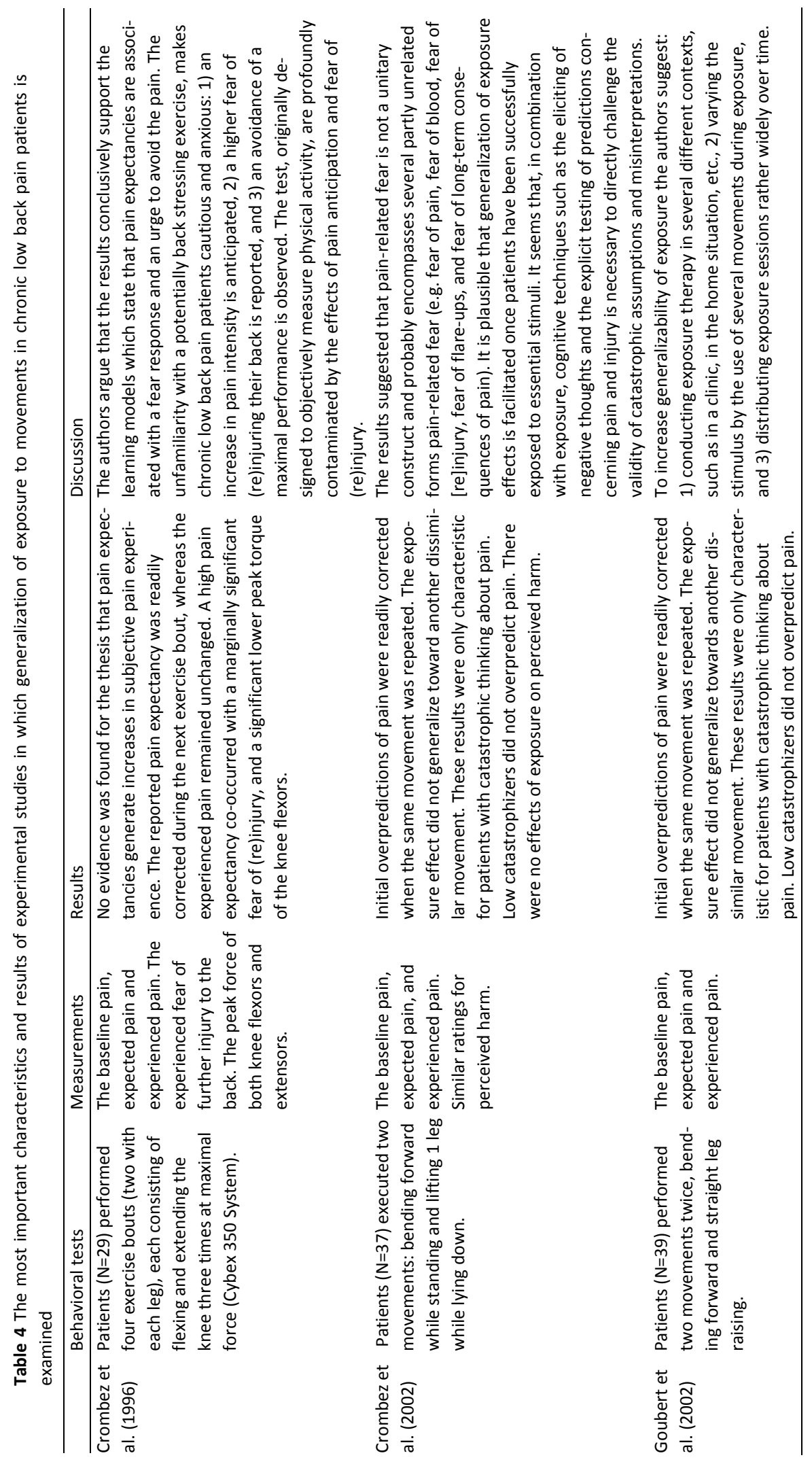




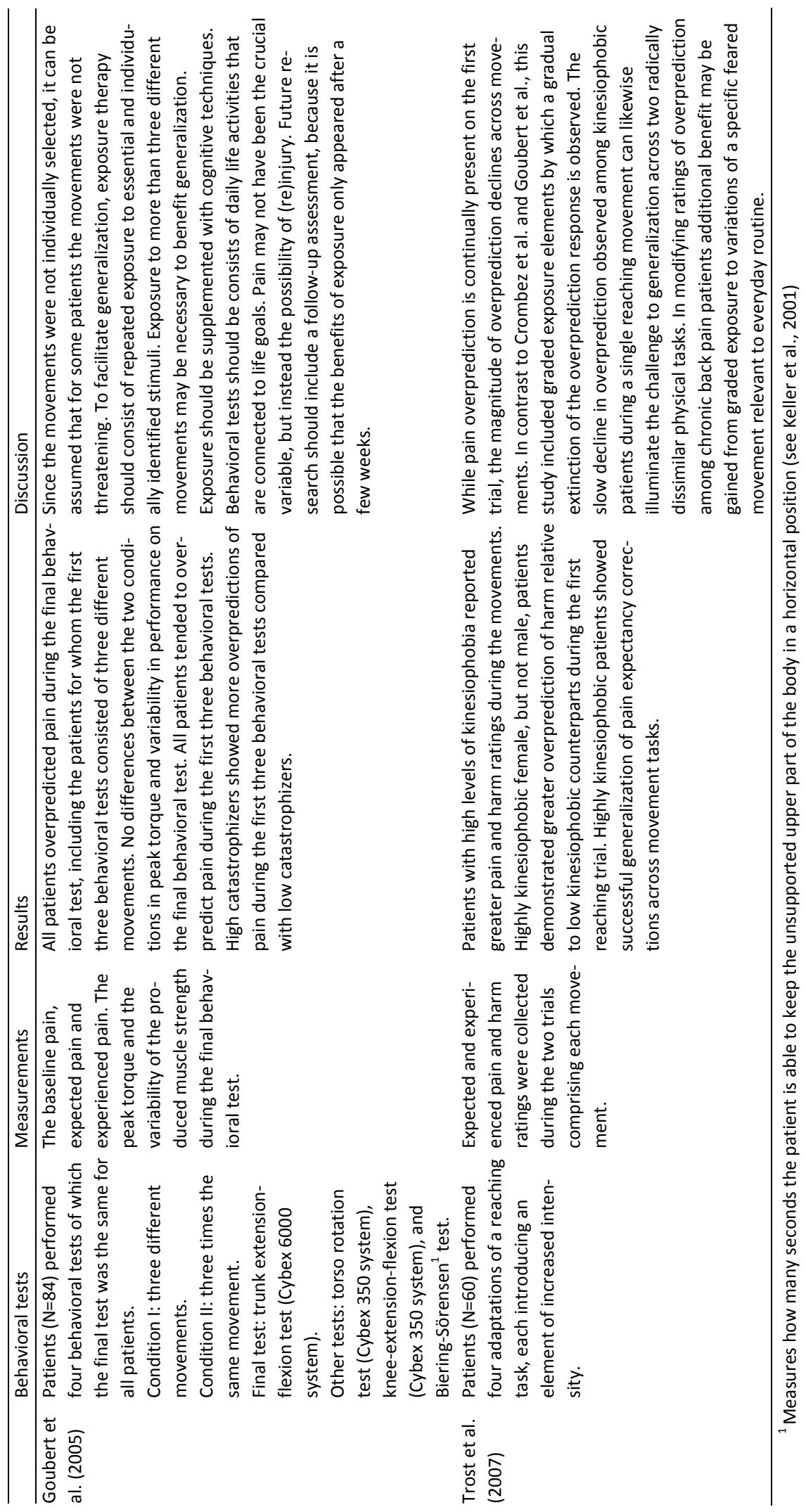


By the use of four exercise bouts (two with each leg), each consisting of flexing and extending the knee three times at maximal force, Crombez et al. (1996) investigated whether pain expectancies cause pain. During each exercise bout the baseline pain, the expected pain and experienced pain were recorded. Patients also rated their experienced fear of further injury to the back. Although patients did expect a back pain increase during the first exercise bout with each leg, no evidence was found for the hypothesis that pain expectancies generate increases in subjective pain experiences. Instead, the reported pain expectancy was readily corrected during the next exercise bout, whereas the experienced pain remained unchanged. This corrective experience generalized, but not completely, to the exercise bouts with the other leg. A small but significant increase in the pain expectancy re-emerged during the first exercise bout with the second leg. A sub-maximal performance was observed in parallel with these pain expectancies. Overall, the results suggest that the unfamiliarity with a potentially back stressing exercise, makes chronic low back pain patients cautious and anxious. First, an increase in pain intensity was anticipated. Second, a marginally significant higher fear of injuring their back was reported. Finally, an avoidance of a maximal performance as indicated by the lesser peak force of the knee flexors, is observed. This pattern of results was present especially during the very first experience with the exercise. Bases on the results it seems that low back pain patients initially tend to over-predict how much pain they will experience, but after some exposures the predictions of pain intensity tend to match with the actual experience.

Crombez et al. (2002) and Goubert et al. (2002) investigated whether the effects of exposure to one movement generalize towards another dissimilar movement. In both studies patients were requested to perform two movements twice, i.e. bending forward and straight leg raising. During each of the four trials baseline pain, expected pain and experienced pain were recorded. Similar ratings for perceived harm were obtained in the study of Crombez et al. (2002). In line with Crombez et al. (1996), analyses of both studies revealed that patients initially over-predicted pain, but after exposure the over-prediction was readily corrected. Thus, it seems reasonable to assume that avoidance behaviors of chronic back pain patients can be limited through defying the harm and danger beliefs about pain and the resulting inaccurate pain expectancies. Given the results it can be assumed that exposure could be an effective method to correct maladaptive beliefs about pain. However, of particular interest to the studies of Crombez et al. (2002) and Goubert et al. (2002) was that although patients readily corrected over-predictions of pain, this correction of over-prediction of pain did not extend across different movements. This means that generalization was not present. Another finding was that the pattern of results was only characteristic for patients reporting a high frequency of catastrophic thinking about pain. Low catastrophizers did not over-predict pain. 
Furthermore, the results of Crombez et al. (2002) seem to indicate that overpredictions of harm were not corrected by exposure.

One explanation of the lack of generalization in the above-mentioned studies might be that patients do not easily change their belief that many movements hurt or harm. Research has shown that such beliefs are widespread in the general population (Goubert et al. 2004). Furthermore, conditioning research has shown that conditioned fear may generalize well across contexts and stimuli, but fear extinction does not (e.g. Bouton \& Swartzentruber, 1991). Thus, the belief that many movements are harmful and painful when you have back pain may be confirmed through exposure to certain pain-provoking activities, which then easily generalizes to other movements. In contrast, extinction of pain-related fear and fear-avoidance beliefs may not easily generalize. Based on the findings that exposure will result in learning an exception of the rule, rather than in a fundamental change of the rule itself, it is thus reasonable to assume that exposure to physical activities and movements in patients with back pain will not result in a fundamental change of the belief that movements are painful and dangerous as predicted, but that other movements are still hazardous and painful. In their attempt to facilitate generalization of the effects of exposure Goubert et al. (2005) investigated the benefits of exposure to a variety of movements versus exposure to the same movements on over-prediction of pain and behavioral performance on a final behavioral test which was the same for all patients. In condition I the first three behavioral tests consisted of three different movements and of three times the same movement in condition II. Replicating and extending previous research, the results showed that patients over-predicted pain during a threatening behavioral test. Furthermore, pain-related fear and pain catastrophizing were unique predictors of the peak torque of the final behavioral test. The study failed to find an advantageous effect of varied-stimulus exposure. This failure may be due to several reasons. First, since the movements were not individually selected, it can be assumed that for some patients the movements were not threatening. To facilitate generalization, it seems that exposure therapy should consist of repeated exposure to essential and individually identified stimuli and more than three different movements. Second, the movements that were chosen for the behavioral tests were highly standardized, by which the tests were somewhat artificial. Finally, pain may not have been the crucial variable which patients over-predicted, but instead the possibility of injury. Although fear of injury was not assessed it is plausible to assume that not fear of pain but fear of injury was affected by exposure to a variety of movements. Patients in condition I may have learned that injury was not very likely since it did not occur during the first three behavioral tests, whereas patients in condition II did not have this opportunity.

Contrary to the previous studies, Trost et al. (2007) showed successful generalization of pain expectancy corrections across movement tasks. In this study the main- 
tenance of corrective experiences were determined across identical movements of increasing intensity in chronic low back pain patients with high versus low levels of pain-related fear. Rather than the series of artificial movements used by Goubert et al., four reaching movements were selected that approximate activities encountered in everyday life. Results indicated that individuals with high versus low levels of pain-related fear reported greater pain and harm, greater over-prediction of harm among female participants, and last but not least an unexpected trend of successful generalization across movements of corrected pain over-predictions. The trend of declining over-prediction was, however, not apparent for harm ratings. Harm over-prediction ratings remained notably stable across movements in participants who reported highly pain-related fear. This finding is not consistent with the assumption of Goubert et al. (2005) who suggested that not fear of pain but fear of injury is affected by exposure. In order to explain for the success of the gradual extinction of the over-prediction of pain, Trost et al. (2007) suggested that the graded exposure elements played an important part.

\section{Summary}

GEXP is developed for application in fearful pain patients. The treatment is highly structured, protocolized, individually tailored, and aims to restore a normal pattern of daily functioning, including complete return to work. Both experimental singlecase studies and randomized controlled trials showed that exposure in vivo is effective in improving functional disability in patients with chronic low back pain. However, in two RCT's this improvement was statistically not significantly better than the control group, who were offered wait-list, usual treatment (contact with a general practitioner, analgesics, and physical therapy) or an operant graded activity. Overall, one of the most striking characteristic in these studies is the discussed importance of the educational component which illustrates and explains the fearavoidance model. Also, the importance of including various fear eliciting activities during exposure and therapeutic competence are described. Finally, the results of the single-case studies as well as those of the RCT's are hopeful with regard to generalization of extinction.

\section{Conclusion and discussion}

Pain is a normal response of the organism when exposed to bodily threat. However, the level of pain someone experience does not necessarily relate to the amount of tissue damage. When pain persists, the danger alarm system in the brain becomes more sensitive. It seems that response systems and thoughts and beliefs become more involved and start contributing to the pain problem. Extremely negative 
thoughts (pain catastrophizing) arouse pain-related fear; fears of interoceptive (e.g. pain), proprioceptive (e.g. movement) and exteroceptive (e.g. physical context) stimuli. Pain-related fear is a strong negative reinforcement for the persistence of avoidance behavior and the functional disability.

The treatment of choice for a number of anxiety disorders is exposure therapy. Experimental studies in psychology showed that extinction of fear is a highly complex process which is probably multiply determined. This complexity is mainly clarified by the observation that extinction is sometimes followed by a (partial) return of fear with the passage of time. Not only is it important to know how exposure treatment can be optimized to reduce the risk of relapse, but from a theoretical perspective it is also crucial to understand the mechanisms by which previously reduced fears reappear.

Exposure can be conceptualized as a clinical analogue of extinction (CS-only presentations after acquisition). Although an extinction procedure results in a decrease of conditioned responses, it does not destroy the underlying CS-US association. What happens is that new associations are added to the existing one. These secondlearned associations are inhibitory, and can be viewed as 'exceptions to the rule' and are likely to be context-dependent. Post-extinction events that might reactivate the original CS-US association are spontaneous recovery, renewal, reinstatement and reacquisition. Moreover, it appears that exposure therapy involve higher-order cognitive processes which are associated with changes in CS-US expectancy. Exposure therapy is also seen as a form of cognitive intervention that specifically changes the expectancy of harm.

Because of the substantial contribution of pain-related fear in the maintenance of chronic pain, it seems to be an important target for intervention. Indeed, a developed exposure program (GEXP) is examined in different exposure studies as an effective treatment in chronic low back pain patients who report high levels of painrelated fear. However, on the basis of the results of these studies and the overall complexity of exposure there are still several questions that need to be worked out, for example, which treatment characteristics makes the GEXP successful or unsuccessful in pain-related fear, what is actually learned during GEXP in pain-related fear, does GEXP in pain-related fear generalize to daily functioning, is GEXP usable in other pain disorders like neuropathic pain? In short, enough questions to answer in the process of optimizing GEXP in pain-related fear.

\section{Outline of this dissertation}

The general theme of this dissertation is to further explore the utility of GEXP in pain-related fear. For this purpose we will examine whether the effects of GEXP 
generalize from chronic low back pain to other pain problems such as complex regional pain syndrome and posttraumatic neck pain. Additionally, the contribution of specific treatment components to outcome will be examined. Finally, the question whether exposure to multiple stimuli enhances generalization after treatment will be addressed. This dissertation comprises five studies and a summary with a general discussion that are organized into the following sections:

First, we will focuses on the role of pain-related fear in neuropathic pain. Painrelated fear recently has received much attention in the pain literature. Numerous studies have shown that pain-related fear is one of the strongest predictors of pain disability, and some have argued that 'pain-related fear is more disabling than pain itself'. However, these studies have mainly been carried out in patients with chronic low back pain, and empirical studies focussing on other chronic pain conditions, such as neuropathic pain are lacking. Recently the International Association for the Study of Pain (IASP, 2005) suggested that the fear-avoidance model may be very relevant in a subset of CRPS patients. Chapter 2 presents two studies, each with a cross-sectional design, in which the role of pain-related fear in Complex Regional Pain Syndrome type I (CRPS-I) is investigated. Study I includes CRPS-I patients visiting an outpatient pain clinic. In study II, members of the Dutch association for CRPSI Patients were invited to complete a number of questionnaires. Besides the role of pain-related fear in predicting disability also various assessments methods of painrelated fear in neuropathic pain are discussed.

Several studies already demonstrated the effectiveness of GEXP in chronic low back pain patients reporting substantial pain-related fear, but its effectiveness was never tested in other musculosketal and neuropathic pain complaints. For patients with acute posttraumatic neck pain (PTNP), pain-related fear has been identified as a potential predictor of chronic disability. If such is the case, fear reduction should enhance the prevention of further pain disability and distress after traumatic neck pain disability. In chapter 3 , a study with a replicated single-case crossover phase design with multiple measurements is presented that examines the effectiveness of GEXP as compared to GA directly and six months after treatment in eight chronic and fearful PTNP patients. Besides the use of a diary and standardized questionnaires of pain-related fears and pain disability also an ambulatory activity monitor was used to quantify daily physical activity level. The results are discussed in the context of the search for customized treatments for PTNP.

The IASP has proposed to test the contribution of pain-related fear in CRPS, and also to examine the effects of an exposure treatment applied in then context of functional restoration in CRPS (IASP, 2005). Following these suggestions, chapter 4, describes a study that examined whether the reduction of pain-related fear through 
GEXP also resulted in a decrease of disability in a subgroup of patients with CRPS-I who report substantial pain-related fear. A single-case experimental $A B C D$-design was used with random determination of the start of the intervention. Eight patients with CRPS-I were included in the study. To assess daily changes in pain intensity, pain-related fear, pain catastrophizing, and activity goal achievement, a diary was used. Standardized questionnaires of pain-related fear, pain disability, and selfreported signs and symptoms of CRPS-I were administered before and after each intervention, and at 6-month follow-up. Possible theories and elements of GEXP that may explain the results of the study are discussed in view of insights from neuroscience.

The results of the first single-case experimental studies examining the effectiveness of GEXP in patients with chronic low back pain conducted by Vlaeyen et al. (2001, 2002a, 2002b) showed abrupt changes in self-reported pain-related fears and cognitions. These changes seem to be more characteristics of insight learning rather than the usual gradual progression of trial and error learning. The educational session at the start of GEXP might have contributed to this insight. In addition, also in the literature about exposure it is suggested that, in order to be effective, exposure must have a convincing rationale (e.g. Barlow, 2004). Chapter 5 presents a study which examined the contribution of education and GEXP versus GA in the reduction of pain-related fear and associated disability and physical activity. Six consecutive patients with chronic low back pain who reported substantial fear of movement/(re)injury were included in the study. After a no-treatment baseline measurement period, all the patients received a single educational session at which an individualized and tailored fear-avoidance model was presented, followed again by a no-treatment period. Patients were then randomly assigned to either GEXP or GA. Besides the use of a diary and standardized questionnaires of pain-related fears and pain disability also an ambulatory activity monitor was used to quantify daily physical activity level. The influence of the education on patients' beliefs and behavior are discussed.

Despite the success of GEXP in chronic low back pain it is still the question whether extinction generalizes to new threatening activities. Research into the return of fear has revealed that extinction does not mean unlearning (e.g. Craske et al., 2008). It appears that the extinguished fear response can arise spontaneously after a while (spontaneous recovery), or reoccur when confronted with a new CS-US event (rapid reacquisition) or an unpredicted US (reinstatement). In addition, it also appears that extinction is very context-sensitive (e.g. Craske et al., 2008). It has been established that what has been learned during the process of extinction will not necessarily generalize towards other situations; not even when the extinction process takes place during the original process of acquisition. In chapter 6 , a study is described in which the primary objective was to replicate the finding that exposure to multiple 
stimuli (or contexts) can enhance the generalization process of extinction. Using a randomized replicated single case experimental design, this study examined whether the effects GEXP in fearful CRPS-I patients generalized to threatening activities which were not addressed during GEXP. Two exposure conditions of 15 sessions were tested: Condition I $(N=4)$ in which GEXP consists of $\geq 15$ activities to which patients were exposed once and Condition II $(N=4)$ of 3 activities to which patients were exposed five times. In both conditions generalization of extinction was tested by exposing patients to a new activity after successful exposure treatment. It was hypothesized that generalization of extinction is facilitated in the condition with exposure to multiple stimuli. Possible mechanisms of GEXP in relation to generalization are discussed.

Finally, in chapter 7, a summary of the main findings is presented together with a general discussion. The findings are positioned within the available literature of extinction, and pain-related fear. Furthermore, the methods and the procedures of the studies are critically appraised, and clinical implications of GEXP as well as recommendations for future research are outlined. 


\section{Chapter 2}

\section{Pain-related fear, perceived harmfulness of activities and functional limitations in Complex Regional Pain Syndrome type I}

Jeroen R. de Jong, Johan W.S. Vlaeyen, Jantha M. de Gelder, Jaap Patijn. Pain-related fear, perceived harmfulness of activities and functional limitations in Complex Regional Pain Syndrome type I. Submitted for publication 


\begin{abstract}
Numerous studies have shown that in patients with chronic musculoskeletal pain, pain-related fear is one of the strongest predictors of pain disability, and the reduction of pain-related fear through an exposure treatment is associated with restoration of functional abilities in patients with low back pain (LBP) and complex regional pain syndrome, type I (CRPS-I). These latter findings suggest that pain-related fear may be associated with functional limitations in neuropathic pain as well. The aim of the current study was to test whether the debilitating role of pain-related fear generalizes to patients with CRPS-I. Two studies are presented. Study I includes a sample of patients with early CRPS-I referred to an outpatient pain clinic. In study II, patients with chronic CRPS and who are members of patients association were invited to participate. The results show that pain severity but not fear of movement/(re)injury as measured with Tampa Scale for Kinesiophobia was related to functional limitations in early CRPS-I. In patients with chronic CRPS-I, however, perceived harmfulness of activities as measured with the pictorial assessment method PHODA significantly predicted functional limitations, together with pain severity. This study supports the idea that pain-related fear in chronic CRPS-I patients is a promising research direction in the understanding of pain disability in CRPS, but that not general fear of movement/(re)injury, but the perceived harmfulness is a key factor that might be addressed more systematically in the assessment of patients with CRPS-I.
\end{abstract}




\section{Introduction}

There is general consensus that the pathophysiology of Complex Regional Pain Syndrome type I is still unknown, and that the contribution of bio-psychological interactions to the development and maintenance of CRPS-I symptoms is unclear (Wilson et al., 2005). During the last decennium, biopsychosocial models have been introduced and successfully applied in chronic pain research (e.g. Turk \& Okifuji, 2002; Vlaeyen \& Morley, 2005). One particular model that has gained wide interest among pain researchers and clinicians alike is the fear-avoidance model. (Vlaeyen \& Linton, 2000; Leeuw et al., 2007c). Patients who (mis)interpret pain catastrophically are likely to become fearful about their pain and its consequences, and engage in protective avoidance behaviours that are adaptive in acute pain, but paradoxically worsen the pain problem in the long run. Although it has repeatedly been shown that in musculoskeletal conditions pain-related fear is associated with ongoing pain and more severe functional limitations, the role of pain-related fear in CRPS-I was never tested systematically. In one study, however, CRPS-I patients responded successfully to an exposure in vivo treatment aimed at the reduction of pain-related fear. This study which employed a replicated single case experimental design, showed that as compared to baseline and education, patients reported not only less pain-related fear but also less pain and increased functional abilities after the treatment (De Jong et al., 2005). These findings are in line with other studies suggesting that differences between nociceptive and neuropathic pain conditions mainly lie in factors that increase pain and beliefs about causes of pain, rather than mood, cognition, and physical function (Daniel et al., 2008). Taken together, these findings suggest that the impact of pain-related fear on functioning is not restricted to patients with nociceptive pain, but is also relevant in patients with neuropathic pain, and CRPS in particular (e.g. Haythornthwaite et al 2003).

Therefore, the aim of the current studies was to test whether the debilitating role of pain-related fear generalizes to patients with CRPS-I. We predicted that pain-related fear is significantly associated with functional limitations, over and beyond the effects of pain severity.

\section{Materials and Methods}

The role of pain-related fear in CRPS-I was investigated in two independent crosssectional studies. Study I used data collected by the pain clinic of the University Hospital Maastricht, pain-related fear was measured with the Tampa Scale for Kinesiophobia (TSK; Miller et al., 1991; Roelofs et al. 2007), a self-report measure focused on the general fear that painful movement might be a sign of (further) injury. In Study II, where data was collected from chronic CRPS-I patients who were mem- 
bers of the Dutch association for patients with CRPS, a pictorial measure of perceived harmfulness of activities (PHODA; Leeuw et al, 2007b) was used in addition to TSK. Methods and results are described in detail below. The Medical Ethics Committee of the Maastricht University approved both studies.

\section{Study 1}

\section{Participants}

In this study, 79 CRPS-I patients were included who visited the outpatient clinic of the Pain Management and Research Center (PKC) of the University Hospital Maastricht for the first time from 2004 to 2006. The diagnosis of CRPS-I was made according to the criteria formulated by Veldman et al. (1993), and based on physician evaluation of objective symptoms. Veldman et al.'s criteria were initially developed for the description of CRPS I in the acute phase of the disease and are formulated as follows: 1) At least four out of five signs or symptoms: pain, difference in skin color, oedema, difference in skin temperature and active range of motion. (2) Signs and symptoms present in an area larger than might be expected of the initial trauma. (3) Increase of signs and/or symptoms during or after exercise.

\section{Procedure}

Once the first appointment at the pain clinic of the University Hospital Maastricht was scheduled, patients were sent a booklet with a series of questionnaires at home. Patients were asked to complete and return the questionnaires a few days before the first meeting with the anaesthesiologist. These data serve as additional diagnostic information which was added to the case history and physical examination. At this point, patients gave their informed consent for using the questionnaire data for research purposes.

\section{Measures}

\section{Sociodemographics}

By means of the above-mentioned booklet, data were available on age, gender and pain duration.

\section{Functional limitations}

One of the most widely used generic health status measures is the Short Form Health Survey (SF-36: Ware \& Sherbourne, 1992). The SF-36 is developed in the late 1980 , and is translated and validated for use among Dutch-speaking residents of the Netherlands (Aaronson et al., 1998). SF-36 is composed of thirty-six questions and standardized response choices, organized into eight multi-item scales (Ware \& Sherbourne, 1992). For this study we used the scale limitations in physical activities 
because of health problems. The raw scale scores were linearly converted to a 0 to 100 scale, with higher scores indicating higher levels of functioning or well-being. The SF-36 has proven to be a reliable and valid instrument, with a mean Cronbach's alpha of 0.84 across scales and samples (Van der Zee \& Sanderman, 1993). Cronbach's alpha of the subscales in our sample of CRPS-I patients was 0.73 .

\section{Pain-related fear}

The Dutch language version of the Tampa Scale for Kinesiophobia (TSK-DV: Goubert et al., 2000) was used. . The TSK-DV measures one's belief that activities which increase pain indicate serious body damage and should be avoided (Goubert et al., 2004; Roelofs et al., 2007). The TSK-DV consists of 17 statements that are rated on a four-point scale ( 1 = strongly agree, 4 = strongly disagree) (Miller et al. 1991). Sample items are "Pain always means I have injured my body" and "If I were to try to overcome it, my pain would increase". The total score was calculated after inversion of items $4,8,12$, and 16 which were phrased in reversed key. The total score varies between 17 and 68 . The Dutch version of the TSK has been shown to be sufficiently reliable and valid (Goubert et al., 2000). Cronbach's alpha of the total score in our sample of CRPS-I patients was 0.94 .

\section{Pain severity}

Four visual analogue scales (VAS), consisting of $10 \mathrm{~cm}$ horizontal lines anchored at the left and the right with the words "no pain at all" and "worst pain experienced" were used (Melzack, 1975). The VAS referred to the present pain, experienced average pain of the last week and the lowest and highest experienced pain. Because the statistical analyses showed the same results for these different VAS, pain severity was described as the result of the experienced average pain (VAS-average pain).

\section{Statistical analyses}

The obtained data were analyzed with the SPSS version 15.0 (SPSS Inc., Chicago, IL). The analyses included pain-related fear and pain severity in predicting functional and role limitations. Multiple regression analyses were carried out with functional limitations as dependent variable. Gender and age were entered into the first step to control for sociodemographic variables. Next, pain-related fear was entered, and pain severity in the third step.

\section{Results Study I}

\section{Sociodemographics}

A summary of the sociodemographics is displayed in table 1. Seventy-nine patients with CRPS-I with an average age of 43.9 years (SD $=13.5$, range $16-80$ ) were included in the analyses. CRPS-I involved the upper extremities in 39\% (4 men and 27 
women), the lower extremities in 44\% (12 men and 23 women), and both the upper as the lower extremities in $16 \%$ ( 1 man and 12 women) of the patients. In total $78 \%$ is female. In $48.1 \%$ of the patients the duration of the CRPS-I symptoms was less than one month, and in $30.5 \%$ the pain duration was between one and six months. The remaining $21.4 \%$ of the patients had pain for longer than 6 months (range 7 months-26 years).

Table 1 The sociodemographics for the patients in Study I and II

\begin{tabular}{lcc}
\hline & $\begin{array}{c}\text { Study I } \\
\text { (N = 79) }\end{array}$ & $\begin{array}{c}\text { Study II } \\
\text { (N = 107) }\end{array}$ \\
\hline Age (years) & & \\
mean & 43.9 & 48.5 \\
standard deviation & 13.5 & 11.3 \\
range & $16-80$ & $23-65$ \\
& & \\
Gender (\% female) & $78 \%$ & $89 \%$ \\
& & \\
CRPS-I upper extremities (\%) & $39 \%$ & $24 \%$ \\
CRPS-I lower extremities (\%) & $44 \%$ & $39 \%$ \\
CRPS-I both extremities (\%) & $16 \%$ & $37 \%$ \\
& & \\
Pain duration & & $1 \%$ \\
$<1$ month (\%) & $48.1 \%$ & $0 \%$ \\
1-6 months (\%) & $30.5 \%$ & $99 \%$ \\
$>6$ months (\%) & $21.4 \%$ &
\end{tabular}

\section{Variable information}

The mean TSK-score, as measure for pain-related fear, was 43.68 (SD $=8.70$, range 20.0-63.0). Compared with normative data about the TSK in chronic low back pain (CLBP) patients (Vlaeyen et al., 1995b) or a mixed group of patients with chronic pain (Nicholas et al., 2008), 52.7\% scored below or on the same level, as the reported median. This suggests that the level of pain-related fear, or more specific fear of movement/(re)injury, of the CRPS-I group in Study I is comparable with the average chronic pain population. The mean group-score of the experienced average pain, as measure for pain severity, was 7.34 (SD = 1.73, range 1.4-10.0). In addition, patients reported for present pain 6.91 ( $S D=2.12$, range 0-10.0), highest experienced pain 8.87 ( $S D=1.07$, range 5.9-10.0), and lowest experienced pain 4.94 (SD = 2.12, range 0.1-10.0).

\section{Regression analyses}

The results of the multiple regression analysis are displayed in table 2. Adding painrelated fear to the model in which age and gender were already included explained additional $19 \%$ of the variance. When pain severity was added to these predictors, 
they accounted for $51 \%$ of the total variance. Step $3(F$ (change) $=18.87, p<.01)$ in the regression model did improve our ability to predict general physical health. Pain severity ( $B=-.479, p<.01$ ) but not pain-related fear (TSK) contributed significantly to the prediction of functional limitations.

Table 2 The results of the multiple regression analysis of Study I $(\mathrm{N}=79)$ with functional limitations as outcome measure

\begin{tabular}{lrc}
\hline & SE B & B \\
\hline Step 1 & & \\
Gender & 6.945 & .150 \\
Age & .226 & -.087 \\
Step 2 & & \\
Gender & 7.426 & .184 \\
Age & .228 & -.081 \\
Pain-related fear (TSK) & .347 & .096 \\
Step 2 & & \\
Gender & 6.716 & .080 \\
Age & .202 & -.082 \\
Pain related fear (TSK) & .308 & .091 \\
Pain severity (VAS-average pain) & 1.556 & $-.479 * *$ \\
\hline$* * p<.01$ & &
\end{tabular}

\section{Conclusion}

In contrast to what was expected, pain-related fear was not a predictor of functional imitations in this sample of early diagnosed CRPS-I patients admitted to the University Hospital. However, fear was measured with the TSK, which might be a too global measure of pain-related fear. A novel measure of pain-related fear is the Photograph Series of Daily Activities (PHODA: Leeuw et al., 2007b; Trost et al., 2009), which more specifically measures the perceived harmfulness of a series of movements and activities presented by photographs. Another explanation could be that the measure of functional disability was too generic and not CRPS-I specific enough. Further, the group of patients who were referred to the pain clinic of the Hospital, might be a highly selected group of CRPS-I patients with early symptoms, and in whom avoidance behaviour might still have adaptive function. Therefore, to examine the role of more CRPS-specific variables for disability and pain-related fear with a different cohort of patients with CRPS-I, Study II was set up.

\section{Study II}

Study II was carried out among members of the Dutch association for Patients of Post-Traumatic Dystrophy (Nederlandse Vereniging van Posttraumatische Dystrofie Patienten: NVPDP). Contrary to study I, this study used more specific measurement 
tools to test the role of pain-related fear and pain severity on functional limitations in chronic CRPS-I patients.

\section{Procedure}

A sample of 400 NVPDP members was randomly selected using the computer program PEPI 3.0 (Abramson \& Gahlinger, 1999), and contacted by mail. A package including patient information, informed consent form, a body pain chart, a letter of recommendation by the patients association and stamped addressed envelopes were sent to them. Body pain charts, developed by A.J. Kresch (http://www.kickas.org/painmap.shtml), were used to indicate the body location where participants felt most severe pain. The instruction was to mark with a cross $(x)$ the body location where the pain was felt as most intense. This information was used to decide which questionnaire(s) should be send to the participants (upper versus lower extremities) to measure functional and role limitations. Depending on the affected limb, different versions of disability instruments were completed.

The participants returned their informed consent forms and the body pain chart in the pre-addressed envelopes to the Maastricht University. The participants also indicated their preference for the online version or the mailed version of the questionnaires. Participants who were able to use the Internet received the online version of the questionnaires by e-mail. The answers were registered and transformed to the computer program Statistical Package for the Social Sciences (SPSS) by the computer program EMIUM (Janssen, 2005). A printed version of the questionnaires was sent by regular mail to the participants who had no access to the internet.

\section{Participants}

Four of the randomly selected NVPDP members lived in foreign countries and were excluded from participation. In total, 396 members were contacted, of which 121 (30.6\%) gave their informed consent to participate. Four of these respondents gave their informed consent too late, one participant was not able to fill in the online version of the questionnaires, another person had filled in the questionnaire, but the sex of this person remained unanswered. Eight participants did not fill in the questionnaires, resulting in a final sample of 107 (27\%) respondents for the purpose of the current analyses.

\section{Measures}

\section{Functional limitations}

The functional limitations were measured by two questionnaires. The first one is the Radboud Skills Questionnaire (RASQ: Oerlemans et al., 2000b), which was completed by CRPS-I patients with upper extremity pain. The RASQ was used to score the patient's ability to perform activities of daily living in the normal manner. The 
activities are described in forty-five items which are divided into six domains: 1) personal care 2) household activities; 3 ) recreational activities; 4) social activities; 5) remaining; and 6) work. Each of the items can be scored on a five-point Likert scale ( $1=$ normal, $5=$ 'I do not perform the activity anymore as a result of CRPS-I'). There is an extra score of ' 9 ' for 'not applicable'. The patient indicates the effort performing the activity by choosing a number. Different scores are computed by summing up relevant items, divided over the number of items of the relevant scale, minus the number of items in which the category 'not applicable' was chosen. A total score can be computed, along with the six domain scores. For this study the total score was converted into a 0-10 score. The questionnaire has been found reliable in CRPSpatients, with good agreement between the outcomes for test-retest and inter observer reliability studies as established with the method of Bland and Altman (1986) (Oerlemans et al., 2000b).

For patients with CRPS-I lower extremity pain, the Dutch questionnaire 'Walking Ability Questionnaire' (WAQ: Roorda et al., 1996a) was used. The three domains that were used in this study are: 1) walking inside the house; 2 ) walking outside; and 3 ) rising and sitting down. The questionnaire is a combination of the original form of the Walking Stairs Questionnaire (WSQ: Roorda et al., 1996b) and a shortened version of the Questionnaire Rising and Sitting Down (QR\&S: Roorda, et al. 1996b). Response options are presented in a dichotomous fashion (yes or no). Following Perez et al. (2002), the score of each of the domains was calculated by adding up the total number of affirmative responses. These scores were converted into a 0-10 total score for each scale, by dividing the score of each scale by the total number of items of that scale, and subsequently multiplying the outcome by 10 . In the case that a patient had marked: 'I do not walk inside/outside due to my dystrophy' the total score of 10 was included for the relevant domain. Items that suggest difficulties with walking due to other reasons than CRPS were excluded from the total score calculation. In terms of test-retest reliability as expressed by Spearmans's $r$ (range $0.79-0.90$ and $0.85-0.89$ respectively) and the intraclass correlation coefficient (ICC: range $0.78-0.84$ and $0.84-0.87$ respectively) the WSQ and the QR\&S form a reliable tool for measuring activity limitation of patients with CRPS-I of a lower extremity. Patients who had lower as well as upper extremity CRPS-I (37\%) filled in both the RASQ and the WAQ.

\section{Pain-related fear}

The Tampa Scale for Kinesiophobia (TSK; Miller et al., 1991), is a brief questionnaire that measures fear of movement/(re)injury. Although psychometric studies have supported the reliability and validity of the TSK (Goubert et al., 2004, Roelofs et al., 2007), limitations are that it does not provide information about other feared stimuli than fear of movement/(re)injury and which specific movements or activities a patient fears or avoids. Therefore, the Photograph Series of Daily Activities (PHODA: 
Kugler et al., 1999; Dubbers et al., 2003; Jelinek et al., 2003) was included as well. The PHODA is an instrument that includes photographs of various daily activities. Patients have to indicate to what extent they perceive these daily activities to be harmful and/or threatening. Support has been found for both the reliability and validity of a computer version of PHODA (Leeuw et al., 2007b).

Two parallel forms of PHODA were used. PHODA-UE (Dubbers et al., 2003) was used for the upper extremities and PHODA-LE (Jelinek et al., 2003) for the lower extremities. For this study a shortened computer version of PHODA-UE and PHODA-LE was developed, consisting of 40 selected pictures. For every basic movement category, activities were selected with variable degrees of rated harmfulness. Participants were instructed to watch each photograph carefully and to try to imagine performing the same activity. To determine the expected harmfulness of these activities a visual analogue scale (VAS), consisting of a $100 \mathrm{~mm}$ horizontal line was used anchored at the left and right by the words 'not harmful' and 'very harmful', was used. Each photograph is given a rating according to its position on the VAS. A mean total score ranging from 0 to 100 is calculated as the sum of each rating divided by 40 . To test the predictive value of the PHODA in the hierarchical regression analyses $\mathrm{PHO}$ DA-UE or PHODA-LE were used depending on the affected limb.

Pain

The Dutch translation of the Neuropathic Pain Scale (NPS: Galer \& Jensen, 1997) was used to assess the distinct pain qualities associated with neuropathic pain. The NPS consisted of ten items. Two items assessed the global dimensions of pain intensity and pain unpleasantness. Seven items contained the words: intense; sharp; hot; dull; cold; and itchy to characterize the patient's pain and the word sensitive to describe the patient's pain reaction to light touch or clothing. In addition, each of these items included a description and other similar descriptive words for that item. One item described the temporal sequence of pain as constant with 'intermittent increases', 'intermittent', or 'constant with fluctuation'. Each item was rated on a 0 to 10 numerical rating scale. Galer \& Jensen (1997) provided preliminary support for the discriminant and predictive validity of the NPS items.

\section{Statistical analyses}

The obtained data were analyzed with the SPSS version 15.0. Because functional limitations were measured differently in patients with CRPS-I in the lower and upper extremities, analyses were performed in two groups: lower extremity group and upper extremity group, by which patients who had CRPS-I in the lower as well as the upper extremity were added to both groups. Pearson correlation showed that the RASQ and the WAQ were highly correlated in the sample of patients who had CRPS-I in both the lower and upper extremity (Pearson $r=.62, p<.01$ ). Descriptive statistics of each questionnaire were generated to evaluate the completeness of the data 
and to characterize the score distributions, including scale ranges, means and standard deviations. In the case of missing data, means replacement methods were used if $90 \%$ of relevant data was present.

Multiple regression analyses were carried out with functional and role limitations as dependent variable. Gender and age were entered into the first step to control for sociodemographic variables. Next, pain-related fear was entered and in the third step pain severity. Regression analyses were repeated in each group separately with the TSK or PHODA as a measure for pain-related fear.

\section{Results Study II}

\section{Sociodemographics}

The sociodemographics for the patients in Study II are displayed in table 1. Hundred-seven members of the Dutch association for patients with CRPS-I (89\% women) with an average age of 48.5 years $(S D=11.3$, range $23-65$ ) were included in the analyses. Fifty-three respondents completed the questionnaires online, fiftyfour respondents completed paper versions of the questionnaires. CRPS-I involved the upper extremities in $24 \%$ ( 6 men and 20 women), the lower extremities in $39 \%$ ( 4 men and 38 women), and both extremities in 37\% ( 2 men and 37 women) of the respondents. The average duration of the CRPS-I symptoms was estimated at 8.14 years (SD $=4.65$, range $0-22$ years). In only one patient the duration of the CRPS-I symptoms was less than one month and no patients reported a pain duration between one and six months. The remaining patients had pain for longer than 6 months.

\section{Variable information}

The mean TSK-score of the patients was 37.69 (SD = 8.20, range 19.0-59.0). Compared with normative data of the TSK in a mixed group of patients with chronic pain, $65 \%$ scored below or on the same level, as the reported median (Nicholas et al., 2008). The mean PHODA-score was 48.02 (SD = 20.66, range 6.25-94.05). Compared with normative data of the PHODA in CLBP patients (Leeuw et al., 2007b), $50 \%$ scored below or on the same level, as the reported median. This suggests that the level of pain-related fear, as measured with the PHODA, of the CRPS-I group in Study II is comparable with the average CLBP population. Furthermore, the mean NPS-score, as measure for pain severity, was 50.16 (SD = 17.46, range 7.00-90.70).

\section{Regression analyses}

\section{Lower extremity CRPS-I group}

Table 3 shows the results of the multiple regression analysis of the lower extremity CRPS-I group in which the TSK was used as measure for pain-related fear. The de- 
mographic variables gender and age were entered in Step 1 and explained 29\% of the variance in functional limitations. The variable pain-related fear entered in step 2 explained an additional $29 \%$ of the variance, and for pain severity in step 3 this was an addition of $44 \%$ of the variance. Inclusion of the predictor pain severity in the model (step 3 ) improved the ability to predict the outcome variable ( $F$ (change) $=9.87, \mathrm{p}<.01)$. Of the variables age $(B=.274, p<.05)$ and pain severity $(B=.351, p$ $<.01)$ were significantly related to the function in which the level of functional and role limitations was predicted.

Table 3 The results of the multiple regression analysis of Study II in lower $(N=79)$ and upper $(N=65)$ extremities CRPS-I patients with functional limitations as outcome measure and the TSK as measure for pain-related fear

\begin{tabular}{lcccc}
\hline & \multicolumn{2}{c}{ Lower extremities } & \multicolumn{2}{c}{ Upper extremities } \\
\hline Step 1 & SE B & $\boldsymbol{B}$ & \multicolumn{1}{c}{ SE B } & \multicolumn{1}{c}{ B } \\
Gender & & & .342 & .103 \\
Age & 1.199 & .235 & .011 & -.141 \\
Step 2 & .026 & .221 & & .107 \\
Gender & & & .344 & -.150 \\
Age & 1.211 & $.234^{*}$ & .011 & .097 \\
Pain-related fear (TSK) & .027 & .222 & .014 & \\
Step 3 & .034 & -.014 & & .023 \\
Gender & & & .262 & .114 \\
Age & 1.164 & .167 & .009 & -.044 \\
Pain-related fear (TSK) & .025 & $.274^{*}$ & .011 & $.717^{* *}$ \\
Pain severity (NPS) & .033 & -.081 & .005 & \\
\hline
\end{tabular}

$* p<.05, * * p<.01$

In an additional multiple regression analysis the PHODA, as measure for pain-related fear, was entered in step 2 (table 4). As a consequence, this explained an additional $48 \%$ of variance in functional limitations. For pain severity in step 3 this was $52 \%$. Unlike the analysis described above, in which the TSK was used as measure for painrelated fear, not the inclusion of pain severity but pain-related fear improved the ability to predict the outcome variable $(F$ (change) $=13.54, p<.01)$. In addition, the multiple regression analysis showed that pain-related fear $(\beta=.328, p<.01)$ and age $(B=.225, p<.05)$, and not pain severity, were significantly related to functional limitations.

\section{Upper extremity CRPS-I group}

Table 3 shows also the results of the multiple regression analysis of the upper extremity CRPS-I group in which the TSK was used as measure for pain-related fear. Adding gender and age in step 1 explained 19\%, pain-related fear in step 2 explained $21 \%$, and pain severity in step 3 explained $68 \%$ of the variance in functional limitations. Inclusion of pain severity in the model (step 3 ) improved the ability to 
predict the outcome variable $(\mathrm{F}$ (change) $=45.43, \mathrm{p}<.01)$. Of the variables only pain severity $(B=.717, p<.01)$ was significantly related to functional limitations.

Table 4 The results of the multiple regression analysis of Study II in lower $(N=76)$ and upper $(N=64)$ extremities CRPS-I patients with functional limitations as outcome measure and the PHODA as measure for pain-related fear

\begin{tabular}{|c|c|c|c|c|}
\hline & \multicolumn{2}{|c|}{ Lower extremities } & \multicolumn{2}{|c|}{ Upper extremities } \\
\hline & SE B & B & $S E B$ & $B$ \\
\hline \multicolumn{5}{|l|}{ Step 1} \\
\hline Gender & 1.232 & .233 & .345 & .099 \\
\hline Age & .028 & .209 & .011 & -.148 \\
\hline \multicolumn{5}{|l|}{ Step 2} \\
\hline Gender & 1.134 & .206 & .299 & .135 \\
\hline Age & .026 & .192 & .010 & -.184 \\
\hline Pain-related fear (PHODA) & .014 & $.395 * *$ & .005 & $.510 * *$ \\
\hline \multicolumn{5}{|l|}{ Step 3} \\
\hline Gender & 1.127 & .172 & .251 & .058 \\
\hline Age & .026 & $.225^{*}$ & .009 & .022 \\
\hline Pain-related fear (PHODA) & .015 & $.328 * *$ & .004 & $.267^{*}$ \\
\hline Pain severity (NPS) & .018 & .221 & .006 & $.575^{* *}$ \\
\hline
\end{tabular}

$* p<.05, * * p<.01$

When the PHODA, as measure for pain-related fear, was entered in step 2 of the regression model (table 4 ) this explained an additional $54 \%$ of variance in functional limitations. Adding pain severity in step 3 this explained $72 \%$. Inclusion of the predictors pain-related fear $(\mathrm{F}$ (change) $=21.05, \mathrm{p}<.01$ ) and pain severity $(\mathrm{F}$ (change) $=$ $27.53, p<.01)$ improved the ability to predict the outcome variable. Contrary to the previous multiple regression analysis, not only pain severity $(B=.575, p<.01)$, but also pain-related fear $(B=.267, p<.05)$ was significantly related to functional limitations.

\section{Conclusion}

Study II showed that the higher pain-related fear, when measured with a pictorial fear of activity scale (PHODA) and not with a self-report measure specifically focused on fear of movement/(re)injury (TSK), the higher impact on the experienced functional limitations. Contrary to Study I the role of pain severity was different in both CRPS-I subsamples. When the PHODA was entered in the regression model in patients who had lower extremity CRPS-I only pain-related fear, and not pain severity, was a significant predictor of functional limitations. 


\section{Discussion}

The primary aim of this study was to investigate the role of pain-related fear in CRPS-I patients. Two studies were carried out. In Study I data of CRPS-I patients who visited an outpatient clinic of Pain Management for the first time were used. Painrelated fear was measured with a self-report measure specifically focused on fear of movement/(re)injury (TSK). The results of Study I showed that pain severity affected functional limitations more than any other variable. In Study II, members of the Dutch association for CRPS-I Patients completed a series of questionnaires. To measure pain-related fear a pictorial fear of activity scale (PHODA) was used. The results demonstrated that pain-related fear, only when measured with a pictorial fear of activity scale, was associated with functional limitations, above and beyond the contribution of pain severity. Moreover, also in Study II, pain severity was a predictor for functional limitations. However, when the PHODA was used in the group of patients with CRPS-I in the lower extremities, only pain-related fear and not pain severity was a predictor of functional limitations. The current studies showed that pain-related fear might affect functional limitations in CRPS-I patients.

To the current knowledge of the authors, both studies are the first to specifically examine the role of pain-related fear in relation to functional limitations in a group of CRPS-I patients. By using motor imagery in CRPS patients Moseley et al. (2008) showed that change in pain and swelling was related to pain catastrophizing and pain-related fear, which they interpreted in a way that pain-related fear affects motor processes and pain even when the individual has no intention to actually execute the movement. Another possible mechanism that is suggested to underlie CRPS-I, and as such may be linked to pain-related fear, is disuse or deconditioning, that develops in an effort to avoid pain (Verbunt et al., 2003). Operantly conditioned disuse of the affected extremity, reinforced by avoidance of actual pain or reduced anxiety of anticipated worsening of pain, may prevent desensitization and eliminate the normal tactile and proprioceptive input from the extremity that may be necessary to restore normal central processing (Bruehl, 2005; Stanton-Hicks et al., 1998). Disuse also suggested to trigger hyperalgesia and allodynia in the affected extremity (Butler, 2001) and to interact with pathophysiological mechanisms that prevent the CRPS-I patient from ending a vicious cycle in which altered central processing leads to increased pain, which provokes catecholamine release that further stimulates the nociceptive input maintaining the central processing alterations (Bruehl, 2001). It is possible that pain-related fear is a mechanism that underlies and contributes to this disuse through its associated avoidance behavior, and that it may help maintain the primary features of CRPS-I. These, of course, are speculations that still await scientific scrutiny. 
Remarkable is that both studies demonstrated that the TSK does not significantly predict functional limitations in patients with CRPS-I. These results are not consistent with previous research among patients with musculoskeletal pain in which pain-related fear was shown to be associated with pain disability, and more so than pain itself (e.g. Crombez et al., 1999; Leeuw et al., 2007a). The TSK has been specially developed for patients with musculoskeletal pain, and back pain in particular. Although there is a lack of consensus among researchers, CRPS-I is thought to be a neuropathic pain disorder (Jänig \& Baron, 2003). Therefore, TSK might not be suitable for measuring pain-related fear in neuropathic pain disorders such as CRPS-I. However, the mean score and the standard deviation of the TSK in Study I did not deviate that much from the scores in studies among chronic back patients, in which the TSK predicts the level of disabilities (e.g. Goubert, 2000).

In contrast to the weak association between TSK and functional disability, pictorial measure of perceives harmfulness of activities (PHODA) appears to be a significant predictor for reported functional limitations in CRPS-I patients. It might be that visualisation of activities makes imagining executing the activity simpler, concrete and salient, as compared to general verbal statements such as the ones used in TSK. Because the PHODA makes performing activities better imaginable, its pictures may elicit more elevated fear levels as compared to the verbal items of the TSK. There is evidence that exposure to pictures with a negative valence elicit fear responses such as increase startle potentiation and also to lower pain tolerance (De Wied and Verbaten, 2002). As PHODA was not included in Study I, we do not know whether perceived harmfulness of activities is also a predictor of disability in patients with early CRPS-I who are admitted to a specialized pain clinic of the University Hospital.

Finally, several limitations of both studies should be mentioned. First, the present studies are limited by there cross-sectional design. Positive correlations or regression weights may not be confused with causal effects. Second, the results are based on a relatively small number of CRPS-I patients. Third, because only $27 \%$ of the initially recruited sample actually participated in the study, there may be any potential impact of selection bias. However, non-response can cause a significant bias even with a high participation rate if the non-responders are a relatively homogeneous group that differs markedly from the responders (Bergstrand et al., 1983). Unfortunately, and due to the study logistics, we do not have information about the characteristics of the non-responders in our study. Fourth, the studies are impeded by the use of self-report measures only, which are subject to bias. Fifth, the two CRPS-I samples differed in duration of the condition. Study I consisted primarily of acute CRPS-I patients and Study II of chronic CRPS-I patients. There is good evidence that pain-related fear is associated with disability in patients with chronic musculoskeletal pain (Vlaeyen \& Linton, 2000). However, also in (sub)acute pain disorders re- 
search showed very clearly that pain-related fear is related to pain and disability, and is a essential factor in the development of chronicity and long-term disability (e.g. Linton, 2000b; Fritz and George, 2002; Swinkels et al, 2003; Heneweer et al., 2007; Vangronsveld et al., 2008). In fact, pain-related fear during the early pain condition seems to be the best predictors of future problems (e.g. Gheldof et al, 2010). Therefore, we do not think that differences in pain duration between both samples may have affected our results adversely. Finally, a further point of consideration is the fact that no gold standard for the diagnosis of CRPS-I has been established, because a single pathophysiological mechanism explaining the variety of features observed in CRPS-I is lacking. Only the criteria set by the International Association for the Study of Pain (IASP) is officially recognized by the IASP to be used for formal diagnosis of CRPS-I (Harden \& Bruehl, 2005). Other sets of diagnostic criteria are from Veldman et al. (1993) and Bruehl et al. (1999). Perez et al. (2007) found that there is a lack of agreement between the different diagnostics sets for CRPS-I and difference in clinical appearance between patients meeting the different criteria which may have profound consequences for the clinical profile of a study population. In study I, the diagnose CRPS-I is made based on the criteria of Veldman and in Study II, the participants declared themselves to be diagnosed with CRPS-I. Despite the choice for any of the criteria sets used in a study remains arbitrary (Perez et al. 2007), the current research results could be better translated if the diagnostic criteria for both studies were uniform.

In summary, the current studies show that pain-related fear, when measured with a pictorial fear of activity scale (PHODA), is a predictor of functional limitations among CRPS-I patients with longstanding pain who are not seeking care in a specialized university pain clinic. Also, it appears that pain severity affects functional limitations in CRPS-I patients. The results indicate that the pain-related fear measures TSK and PHODA assess unique constructs of fear. However, it is not clear what CRPS-I patients with a high pain-related fear score, actually fear. Study I and II provide data to start a qualitative study to address the current concerns of CRPS-I patients. Despite the need replication in a larger CRPS-I sample, this study supports the idea that pain-related fear in CRPS-I patients is a promising research direction to get more insight in a poorly understood painful and disabling disorder (Nelson, 2002).

What are the clinical implications of the current studies? If pain-related fear is indeed such a relevant factor it could be a treatment target in CRPS-I. Treatments that are based on the fear-avoidance model of pain do not primarily aim at pain relief, but at extinguishing pain-related fear and the presumed associations patient may have learned between certain activities and/or movements and potential body harm. Because of remarkable similarities between pain-related fear and fear/phobias in general, and the success of exposure-based treatments in patients 
with anxiety disorders, a similar treatment was developed for application in fearful chronic pain patients (Vlaeyen et al., 2004). A preliminary experimental study of de Jong et al., (2005) supported the effectiveness of a graded exposure in vivo approach to chronic CRPS-I. The most essential step in this approach consisted of graded exposure to the situations the patients has identified as 'dangerous' or 'threatening'. Thus, in chronic CRPS-I patients it seems appropriate to assess not only pain severity, but also pain-related fear for which a treatment approach is available that shows promising results for this disabling chronic pain condition (see Baily et al., 2010 for a review).

\section{Acknowledgements}

This study was supported by the foundation Esperance related to the Dutch association for Patients of Post-Traumatic Dystrophy (Nederlandse Vereniging van Posttraumatische Dystrofie Patienten) and the department of Pain Management and Research of the University Hospital Maastricht. We are grateful for the efforts of the volunteers of the Dutch association for Patients of Post-Traumatic Dystrophy, to Rosanne Janssen who designed the computer program EMIUM which was very helpful in the realization of Study II, to Eric Schouten who helped with the statistical analysis, to Elke Vermeulen for her assistance in the data collection of Study I, and to all participants filling in the questionnaires. Participation of Johan W.S. Vlaeyen was supported by the NWO Social Sciences Research Council of the Netherlands, VICl Grant No. 453-04-003, and an Odysseus Grant by the Fund for Scientific Research (FWO) - Flanders, Belgium. 



\section{Chapter 3}

\section{Reduction of pain-related fear and disability in posttraumatic neck pain: a replicated single case experimental study of exposure in vivo}

Jeroen R. de Jong, Karoline Vangronsveld, Madelon L. Peters, Mariëlle E.J.B. Goossens, Patrick Onghena, Isis Bulté, Johan W.S. Vlaeyen. Reduction of pain-related fear and disability in posttraumatic neck pain: a replicated single case experimental study of exposure in vivo. The Journal of Pain 2008; 9(12): 1123-1134. 


\begin{abstract}
For patients with acute post-traumatic neck pain (PTNP), pain-related fear has been identified as a potential predictor of chronic disability. If such is the case, fear reduction should enhance the prevention of further pain disability and distress after traumatic neck pain disability. However, exposure-based treatments have not been tested in patients with PTNP. Using a replicated single-case crossover phase design with multiple measurements, this study examined whether the validity of a graded exposure in vivo, as compared with usual graded activity, extends to PTNP. Eight patients who reported substantial pain-related fear were included in the study. Daily changes in pain intensity, pain-related fear, pain catastrophizing, and activity goal achievement were assessed. Before and after each intervention, and at 6month follow-up, standardized questionnaires of pain-related fear and pain disability were administered, and, to quantify daily physical activity level, patients carried an ambulatory activity monitor. The results showed decreasing levels of selfreported pain-related fear, pain intensity, disability, and improvements in physical activity level only when graded exposure in vivo was introduced, and not in the graded activity condition. The results are discussed in the context of the search for customized treatments for PTNP.
\end{abstract}

\title{
Perspective
}

This is the first study showing that the effects of graded exposure in vivo generalize to patients with chronic PTNP reporting elevated levels of pain-related fear. This could help clinicians to customize treatments for PTNP. 


\section{Introduction}

An increasing number of both experimental and clinical studies, mainly performed in patients with chronic low back pain (CLBP), have shown that pain-related fear is one of the most potent predictors of observable physical performance, selfreported disability levels in daily life situations, and work loss (Buer \& Linton, 2002; Fritz et al., 2001; Leeuw et al., 2007a; Vlaeyen \& Linton, 2000). The basic tenet of the fear-avoidance model of pain is that the way in which pain is interpreted may lead to 2 different pathways. When dysfunctional beliefs about pain exist, a number of safety behaviors are initiated that may be adaptive in acute pain but paradoxically worsen the problem in the case of long-lasting pain. Typical safety behaviors are avoidance/escape behaviors and hypervigilance and the prolonged use of them maintains the fear level rather than reduces it. Fearful patients have a risk of getting mired in a downward cycle of pain, fear, avoidance, and increased disability. In contrast, when acute pain is perceived as less threatening, patients are likely to maintain their engagement in daily activities, through which functional recovery is promoted (Asmundson et al., 1999; Vlaeyen \& Linton, 2000). The fear-avoidance model has been successfully tested in patients with back pain (Picavet et al., 2002; Swinkels et al., 2006; Turner et al., 2006), osteoarthritis (Heuts et al., 2004), and burn injuries (Sgroi et al., 2005).

If pain-related fear is indeed one of the important mechanisms responsible for the development and maintenance of chronic pain disability, fear reduction should enhance the prevention of further pain-disability and distress. Well-designed procedures exist for the treatment of specific fears and phobias and usually these involve repeated and systematic exposure to fear-provoking stimuli, often presented in the context of behavioral experiments (Barlow, 2002; Butler et al., 2006; Clark, 1986). Although Philips (1987) argued for the application of exposure techniques to chronic pain some time ago, the first systematic experimental studies and randomized, controlled clinical trials in patients with chronic back pain were carried out more recently (Boersma et al., 2004; De Jong et al., 2005; Leeuw et al., 2008; Linton et al., 2008; Vlaeyen et al., 2001; Vlaeyen et al., 2002a; 2002b; Woods \& Asmundson, 2008).

In patients with post-traumatic neck pain (PTNP) pain-related fear is also found to be an important predictor for chronic disability (Nederhand et al., 2004; 2006). Given the beneficial effects of cognitive behavioral interventions for chronic pain (Morley et al., 1999), cognitive behavioral therapy programs have been developed for patients with PTNP disability as well (Foster et al., 2003; Jaspers, 1998; Linton \& Andersson, 2000a; Linton \& Ryberg, 2001; Soderlund \& Lindberg, 2001; Sterner et al., 2001), of which those promoting physical activity have proven to be the most 
effective (Crawford et al., 2004; Provinciali et al., 1996; Rosenfeld et al., 2003; Soderlund \& Lindberg, 2001; Vassiliou et al., 2006; Vendrig et al., 2000). Although these studies suggest that activity increase is associated with faster return to work and a decrease in pain and disability levels, there is evidence showing that these changes are mediated by the reduction of the catastrophic (mis)interpretations of pain (Mannion et al., 1999; Smeets et al., 2006a; Spinhoven et al., 2004). Therefore, we decided to test the effectiveness of an intervention that has catastrophic interpretations and associated pain-related fear as its primary target. Given the beneficial results of graded exposure in vivo (GEXP) in patients with CLBP, and since painrelated fear has shown to be associated with neck pain injury (Nederhand et al., 2004), there are good reasons to believe that an GEXP treatment would be beneficial for the PTNP population as well.

Using a replicated crossover, single-case, experimental phase design with multiple measurements, we examined whether the validity of a GEXP, as compared with a usual operant graded activity program (GA), extends to patients with chronic PTNP disability. We expected that GEXP would be superior to GA in patients reporting elevated levels of pain-related fear.

\section{Materials and Methods}

\section{Study Design}

A sequential replicated crossover, single-case, experimental phase design was used. This design contains both direct and systematic replication elements to examine the effectiveness of GEXP as compared with GA. Direct replication is replication of the same experiment with another patient. Patients were randomly assigned to 1 of the 2 conditions. Randomization occurred after the 14 baseline days (BAS) and was done by a computer system, providing allocations in a file that could be assessed only by an independent research administrator. In condition I, patients received GEXP first, followed by GA. In condition II, the sequence of treatment modules was reversed.

\section{Participants}

Eight consecutive patients who had chronic neck pain ( $>12$ weeks) after a motor vehicle accident were included in the study. All patients were diagnosed as having whiplash-associated disorder (WAD), resulting from an acceleration-deceleration mechanism of energy transfer on the cervical spine. On the basis of these predetermined inclusion and exclusion criteria, the present study focused on patients 
with grades I (neck pain but no physical findings) and II (pain and musculoskeletal findings such as reduced cervical range of motion) as decreed by the grading system of the Quebec Task Force on WADs associated with motor vehicle collisions (Spitzer et al., 1995). Patients with signs of a concussion, retrograde, or post-traumatic amnesia, serious injuries (eg, fractures, traumatic internal organic pathology), and any neurological signs were excluded. Two of the 8 participants reported memory problems, 3 reported problems concentrating, and 4 reported neither problems in memory or concentration. Besides the above-mentioned other exclusion criteria were illiteracy, pregnancy, alcohol or drug abuse, non-Dutch-speaking, and serious psychopathology. To check the latter, preset criteria based on Dutch norms were applied on the Symptom Checklist (SCL-90: Arrindell \& Ettema, 1986). Because of post-traumatic amnesia and non-Dutch-speaking, 2 potential participants were excluded from the study. With regard to psychopathology none of the potential participants were excluded. The sample consisted of 5 male and 3 female patients, with a mean age of $45 \pm 10.30$ (SD) years and a mean duration of pain disability of 44.4 months (range, 27.6-67.2 months). The patients were referred for outpatient behavioral rehabilitation at the department of rehabilitation of the Maastricht University Hospital or the Hoensbroek Rehabilitation Center and reported substantial fear of movement/(re)injury (Tampa Scale for Kinesophobia [TSK: Kori et al., 1990] score $\geq 40$ ).

The Medical Ethics Committee of the Rehabilitation Foundation Limburg-Institute for Rehabilitation Research Hoensbroeck approved the research protocol in addition to the institutional committee of the University Hospital Maastricht.

\section{Procedure and Program Overview}

Patients were first evaluated by the rehabilitation physician, who conducted a full physical examination, evaluated previous diagnostic tests, and who informed participants about the study. When patients agreed to participate, the researcher sent additional written information, along with an informed consent form, TSK, and SCL90. If patients scored $\geq 40$ on the TSK and fulfilled the preset SCL-90 criteria, they were invited for an intake procedure.

During the intake procedure, information was gathered to complete a behavioral analysis of the pain problem with special attention to the patient's catastrophic interpretations of his/her pain problem. At the end of the interview, the therapist encouraged the patient to formulate specific treatment goals, preferably in terms of activities that had been avoided such as household chores, leisure, or work activities. A hierarchy of fear-eliciting movements and activities was made using the Photograph series of Daily Activities for the upper extremities (PHODA: Dubbers et al., 2003), a standardized method during which patients are requested to judge the harmfulness of 125 diverse physical movements from daily life activities repre- 
sented by photographs. Using a (fear) thermometer, each picture is given a rating between zero (representing the situation which is not harmful for the neck) to 100 (representing the situation that is absolutely damaging the neck). Various forms of PHODA have been used successfully in previous studies (De Jong et al., 2005; Leeuw et al., 2008; Vlaeyen et al., 2001; 2002a; 2002b).

After this assessment, patients started with a no-treatment 2-week BAS. During the second week of BAS, patients wore ambulatory accelerometry-based activity monitors to register daily activity levels. After this first period, the 8 patients were randomly allocated to 1 of 2 intervention sequences, GEXP followed by GA or vice versa. GA consisted of 20 sessions of 1 hour during 10 weeks. GEXP consisted of 12 sessions of 1 hour during 6 weeks. After termination of GEXP and GA, patients carried the activity monitor for 1 week with the instruction to resume their daily activities as much as possible. The fourth period was a 6-month follow-up (follow-up) at the end of which patients once more carried the accelerometry-based activity monitor for 1 week. During BAS, GEXP, GA, and follow-up, patients completed daily measures at home. Questionnaires were completed before and after BAS, after GEXP, after GA, and at follow-up.

\section{Interventions}

Two different outpatient therapist teams provided GA and GEXP. Both teams consisted of a behavioral therapist and an occupational or physical therapist experienced in the cognitive-behavioral rehabilitation of patients with chronic pain. GEXP and GA are highly structured, protocolized, and individually tailored and aim to restore a normal pattern of daily function, including complete return to work. Pain reduction is not a direct goal of either intervention.

\section{Graded Exposure In Vivo}

The GEXP consists of several components: Goal identification, education, exposure in vivo, and generalization.

\section{Goal Identification}

First, the patient is invited to formulate his or her own treatment goals. The therapist makes clear that GEXP does not primarily aim at reducing pain but at the restoration of functional abilities despite pain. Subsequently, the patient and therapist agree on one or more realistic and specific goals that are formulated in positive terms. Activities (e.g., lifting weights) that are in line with these goals (e.g., return to work) are those that will be included in the graded exposure sessions. 


\section{Education}

Patients are given a careful explanation of the fear-avoidance model (Vlaeyen 1995a; 1995b), using their own individual symptoms, beliefs, and behaviors in relation to their pain complaints. The therapist illustrates the paradoxical and dysfunctional effects of avoidance as safety behavior and offers the patient a new view on pain as a common condition that can be self-managed rather than as a serious disease or a condition that needs careful protection. One of the major goals of the educational component is to help the patient understand that the consequences of pain are catastrophically overestimated.

\section{Exposure In Vivo}

Individually tailored practice tasks are developed based on the graded hierarchy of fear eliciting activities and/or movements. The exposure takes the form of a series of behavioral experiments in which dysfunctional beliefs are explicitly being challenged. These assumptions take the form of "If . . . then . . ." statements (eg, "If I lift up my child, then nerves in the neck region will rupture and my muscles will get blocked") and are empirically tested during a behavioral experiment.

\section{Generalization}

To enhance generalization and maintenance exposure is provided to the full spectrum of contexts and natural settings in which fear has been experienced, and the stimuli are varied. For example, bicycling can be done on a city bike and/or mountain bike, uphill as well as downhill, on rough as well as even terrain, and so on. The exposure procedure included activities from PHODA and other activities. A more detailed description of GEXP can be found in Vlaeyen et al. (2002; 2004).

\section{Graded Activity}

The GA is based on the programs originally described by Fordyce (1976) and updated by Sanders (2002). The main goal of GA is the systematic removal of the contingent relationship between overt pain behavior and its positive consequences. This implies that GA is guided by the patient's functional abilities and a timecontingent rather than a pain-contingent regimen. In this study, GA consisted of the following components: Education, identification of goals, establishment of a baseline, successive approximation, and generalization.

\section{Education}

The educational session is similar to the one in GEXP, except that the focus is on the detrimental effects of inactivity and not on dysfunctional beliefs. 
Identification of Goals

Similar to GEXP, realistic and functional treatment goals are formulated based on the patient's main complaints. Goals are split up into separate activities in the quota system.

Establishing Baseline Levels

For each of these activities, a baseline level is determined based on a paincontingent principle ("go on with this activity until your pain makes you feel like discontinuing"). Afterward, time-contingent treatment quotas for each activity are developed, always starting below the mean baseline value.

\section{Successive Approximation}

During the treatment phase, the patient systematically increases the timecontingent quotas to enable him/her to reach his/her personal goals within the preset therapy time period. The patient practices at home and documents every activity or exercise on a performance chart. These charts are discussed in each treatment session, and all team members positively reinforce the individual progress and successive approximations towards predefined (sub)goals.

\section{Generalization}

At the end of the treatment, activities are planned outside the hospital, preferably in the home and work setting to enhance response generalization. A more detailed description of GA can be found in Sanders (2002).

\section{Outcome Measures}

The primary outcome measures are self-reported achievement of functional goals, and pain disability. Secondary outcome measures are pain catastrophizing, painrelated fear, physical activity levels in the home situation, and pain intensity.

\section{Daily Diary Measures}

To check whether the GEXP and/or GA indeed modified activity goal achievement, pain-related fear, and pain intensity, a brief diary was used consisting of 14 items with visual analog scales (VAS). The first 11 items (Table 1) represented the main factors of existing questionnaires for fear of movement/(re)injury (TSK: Goubert et al., 2004; Kori et al., 1990; Roelofs et al., 2004), fear of pain (Pain Anxiety Symptoms Scale; PASS: McCracken et al., 1992; 1999), and pain catastrophizing (Pain Catastrophizing Scale; PCS: Sullivan et al., 1995; Van Damme et al., 2002). All items were scored on 10-cm VAS, anchored "totally disagree" to "totally agree." Three main scores were derived, consisting of the mean scores (range, 0-10) of the items from the TSK, PASS, and PCS. Pain intensity was measured with an additional VAS an- 
chored with "no pain at all" at one extreme and "worst pain experienced" at the other. The last 2 VAS referred to the performance of personally relevant activities that represented 2 main functional goals. Each scale was preceded by the same question: "How difficult was it to perform this activity today?" The scale was anchored with "no problem at all" at one extreme and "impossible" at the other. The diary was completed during the whole duration of the study, and the follow-up period of 1 week. The patients were requested to complete the diary each evening and to send the package by mail to the researchers the next day. The diary has been shown to be sensitive to GEXP in previous studies (De Jong et al., 2005; Vlaeyen et al., 2001; 2002a; 2002b).

\section{Functional Disability}

The Neck Disability Index (NDI) is a 10-item self-reporting instrument for the assessment of physical disability of subjects with neck pain, particularly from whiplash-type injuries (Vernon \& Mior, 1991). Each item is scored from 0 to 5 . The NDI has been shown to have a high degree of test-retest reliability, internal consistency, and acceptable level of validity being sensitive to severity levels and to changes in severity over time (Riddle \& Stratford, 1998; Vernon \& Mior, 1991. Disability categories for the NDI are: 0 to $4=$ no disability, 5 to $14=$ mild disability, 15 to $24=$ moderate disability, 25 to 34 = severe disability, and above 34 = complete disability. We used a Dutch version, which has shown to be a reliable and responsive instrument in patients with acute neck pain in general practice (Köke et al., 1999; Vos et al., 2006).

Table 1 Items of the Shortened and Adapted Versions of the TSK, PASS, and PCS That Are Completed on a Daily Basis

Fear of movement/(re)injury (adapted and modified from TSK)

1. If I exercise I might be in danger of reinjuring myself (Harm)

2. My body is telling me I have something dangerously wrong (Fear)

3. My pain complaints would decrease if I were to exercise (Exercise)

4. I can't do everything because it's too easy for me to get injured (Avoidance)

Fear of pain (adapted and modified from PASS)

1. I become sweaty when in pain (Somatic anxiety)

2. I feel confused when I hurt (Cognitive anxiety)

3. When I feel pain, I think that something dreadful may happen (Fear)

4. When I feel pain I try to stay quite as possible (Escape/Avoidance)

Pain catastrophizing (adapted and modified from PCS)

1. When I am in pain I keep thinking about how badly I want the pain to stop (Rumination)

2. When I am in pain I wonder whether something serious may happen (Magnification)

3. When I am in pain I feel I can't go on with my daily activities (Helplessness)

Abbreviations: PASS, Pain Anxiety Symptoms Scale; PCS, Pain Catastrophizing Scale; TSK, Tampa Scale for Kinesophobia. 


\section{Pain-Related Fear}

The complete Dutch version of the TSK was used. This questionnaire consists of 17 items, measuring fear of (re)injury due to movement, scored on a 4-point scale. The TSK has been found reliable and valid and was capable to predict chronic disability in neck pain (Nederhand et al., 2004; 2006).

\section{Physical Activity Level}

To objectively assess physical activity level (PAL) in the home situation of the patients, patients carried a CSA/MTI uniaxial accelerometry-based electronic activity monitor (Computer Science and Applications, now Manufacturing Technology Incorporated, Fort Walton Beach, FL). The monitor is attached to a belt dorsally, at the height of the thoracic vertebras and uses a built-in single axis accelerometer designed to detect normal human motion. The monitor outputs movement counts, which reflect the summation of vertical accelerations from 0.05 to $2.1 \mathrm{G}$. Data was stored for 1 week. The subjects wore the accelerometer during the daytime except during water-based activities. The data were downloaded to a computer via an infrared interface for data processing. Raw data were exported in 1-minute intervals and saved in separate files for each subject. Patients kept a notebook daily in which they registered the time carrying the activity monitor and the kind of activities performed. Total physical activity was expressed as total counts divided by registered time; counts $\cdot \min ^{-1} \cdot d^{-1}$ (counts per minute of days registered). The activity monitor used in our study appeared to have acceptable reliability for most research applications (Welk et al., 2004).

\section{Manipulation Check}

To check whether the threat value of physical activities was diminished as a result of GEXP, the PHODA for upper extremities was repeated after baseline, GA, and GEXP. Each photograph is given a rating according to the position on the fear thermometer. A total score ranging from 0 to 100 is calculated as the sum of each rating, divided by 125 (the maximum total score).

\section{Validity Checks}

To avoid contamination the GEXP and the GA were given by different experienced therapists. In addition and to avoid contamination as a result of patient interactions, patients randomly assigned to the different conditions received their treatments at different days. Finally, records of activity performances, according to graded hierarchies or preset quota, were kept to enhance the compliance of the patients. 


\section{Statistical Analyses}

Besides graphical interpretations for analyzing the data of the daily measures, a randomization test for single-case experimental phase designs, based on the random determination of the moments of phase change or intervention points, was carried out (Edgington, 1975; 1980; Ferron \& Onghena, 1996; Onghena, 1992; Onghena \& Edgington, 2005). With respect to Student $t$ tests, analysis of variance $F$ tests, or other inferential procedures from within the general linear model framework, randomization tests have the advantage of being valid for single-case experiments without making distributional assumptions (Edgington, 1980; Edgington \& Onghena, 2007; Onghena \& Edgington, 2005), of being easy to apply (Edgington, 1980; Onghena \& Edgington, 2005), and of being extremely versatile for even the most complex single-case designs (Onghena \& Van Damme, 1994; Persons \& Silberschatz, 1998). A randomization test is a permutation test based on random assignment to test a null hypothesis about treatment effects in a randomized experiment (Edgington, 1975; 1980; 1992; Edgington \& Onghena, 2007). The randomization tests for the different single-case designs all make use of a directional test statistic (a difference between means). Replicated single-case experiments may be considered as multiple studies that can be combined using meta-analytical procedures. In the current study we used $P$ value combining, which has the advantages that it is broadly applicable and that it is distribution-free without converting the scores to ranks or signs (Edgington, 1992; Onghena \& Edgington, 2005). A more detailed description of the randomization tests for single-case experimental designs and sequential replication designs in particular can be found in Onghena and Edgington (2005). Because GEXP was expected to be superior to BAS and GA, the null hypothesis that there is no differential effect for any of the measurement times was tested using a randomization test on the differences between GEXP and BAS, GA and BAS, and GA and GEXP. Although follow-up is expected to be superior to BAS and will not change in relation to GEXP, differences between follow-up and BAS, follow-up and GA, follow-up and GEXP were also tested using randomization tests. The analysis is performed using the SCRT software (Single-Case Randomization Tests, version 1.1; Katholieke Universiteit Leuven, Leuven, Belgium, 1994). Finally, the test is repeated, assuming delayed effects until the minimal $P$ value $(P<.05)$ is reached (Edgington, 1992; Edgington \& Onghena, 2007; Wampold \& Furlong, 1981). One effect lag equals 1 week, or 2 treatment sessions.

\section{Preset Criteria for Nondaily Measures}

For the nondaily measures, the limited number of data made it impossible to use randomization tests. Therefore, we decided to formulate preset criteria to conclude whether the treatment could be considered successful. For the NDI, a 5-point change is required to be clinically meaningful (Stratford et al., 1999). For the TSK 
and PHODA, we considered a $50 \%$ decrease would give enough support that the threat value of the activities had decreased. This decision was based on the results of exposure studies of patients with CLBP who show at least a comparable decrease for these variables (De Jong et al., 2005; Vlaeyen et al., 2001; 2002a; 2002b).

\section{Results}

\section{Manipulation Check}

The results of the PHODA for upper extremities, used to check whether the threat value of physical activities has diminished as a result of GEXP, are summarized in Table 2. In condition I, as compared with the start of GEXP (PHODA score $=86$ ), a relevant reduction ( $\geq 50 \%$ decrease) in PHODA-scores is observed at the end of GEXP (PHODA score $=9$ ), and there was no further reduction during GA (PHODA score $=7$ ) and follow-up (PHODA score $=8$ ). In condition II, the PHODA score decreased somewhat from 85 at the start of GA to 68 at the end of GA. However, once again, when GEXP was introduced, the PHODA score decreased further to 8 ( $\geq 50 \%$ decrease) and remained at the same level at follow-up.

Table 2 Mean Scores (Range) for Pain-Related Fear (TSK and PHODA) and Pain Disability (NDI), Determined at Baseline, Before, and After Each Treatment Module, and at the 6-Month Follow-Up for Condition I $(n=4)$ and Condition II $(n=4)$

\begin{tabular}{lccc}
\hline Condition Interval & NDI (0-50) & TSK (17-68) & PHODA (0-100) \\
\hline Condition I & & & 83 \\
Baseline & 37.8 & 47.5 & 86 \\
Start GEXP & 37.8 & 47.5 & 9 \\
End GEXP & 7.5 & 24 & 9 \\
Start GA & 7.5 & 24 & 7 \\
End GA & 7.5 & 24 & 8 \\
6-mo follow-up & 8.5 & 25 & 85 \\
Condition II & & & 85 \\
Baseline & 35.5 & 48 & 68 \\
Start GA & 35.5 & 48 & 69 \\
End GA & 27 & 41 & 8 \\
Start GEXP & 27 & 41 & 8 \\
End GEXP & 8.5 & 23 & 23 \\
6-mo follow-up & 8.5 & 23 & \\
\hline Abbreviations: TSK & & & \\
\end{tabular}

Abbreviations: TSK, Tampa Scale for Kinesophobia; PHODA, Photograph series of Daily Activities for the upper extremeties; NDI, Neck Disability Index; GEXP, graded exposure in vivo; GA, graded activity. 


\section{Daily Measures}

Because the patterns of change for fear of movement/(re)injury, fear of pain, pain catastrophizing, and pain experience of each patient within both conditions are quite similar, we decided to calculate group means of the time series for these variables. This produced more conveniently arranged graphs. Figure 1 displays the graphical representations for fear of movement/(re)injury, pain experience, fear of pain, and pain catastrophizing. Visual inspection reveals that in both conditions trend changes occur after the introduction of GEXP only and that these changes are still present during the 6-month follow-up period. By contrast, the introduction of GA does not lead to observable trend changes. These observations suggests that, for chronic neck pain patients who reported substantial pain-related fear, fear of movement/(re)injury, pain experience, fear of pain, and pain catastrophizing are reduced only by GEXP. It is also remarkable that at the onset of GEXP in both conditions pain experience increased. There appears to be an increase in pain experience at the start of the sessions before the standard decrease observed in sessions 7 through 9. In fact, in condition II, this increase in pain appears to return to levels that were experienced at the start of the GA protocol. Also, in both conditions, the results suggest that the decrease in pain experience temporarily follows an associated decrease in fear of movement/(re)injury.

The graphical representations of personally relevant activities for both conditions are displayed in figure 2 . Because both selected activities show the same patterns of change for each patient, only 1 activity per person is presented. Again, only substantial trend changes are observed when GEXP is introduced.

The results of the randomization tests on the raw data of the daily measures confirm the conclusions of the graphical display. For the variables fear of movement, fear of pain and pain catastrophizing the moments of phase change or intervention points in which significance is reached take place during the GEXP (Table 3). Because the above-mentioned variables show the same results ( $P$ values) for each patient only the variable fear of movement is presented. For all patients in condition I (BASGEXP-GA-follow-up), the effect lag during GEXP, in which the minimum $P$ values $(P>$ .05) for the randomization tests was reached, is the fifth week. In condition II (BASGA-GEXP-follow-up), this is the same for patient 1 and 5. Conversely, for patient 6 and 8 the minimum $P$ value was reached in the fourth week of GEXP. With regard to pain experience and the performance of personally relevant activities such as constructing a floor, gardening, mountain biking/jogging, working as a nurse/salesman, playing with the children, looking backward, and dancing, significant moments of phase change $(P>.05)$ occurred only during GEXP, in both conditions in the fifth week (Table 3). For all variables, the measurement periods after the GEXP did not 
provide any other significant phase changes with regard to a positive improvement or a possible relapse.

\section{Condition I (Baseline-GEXP-GA-FU)}

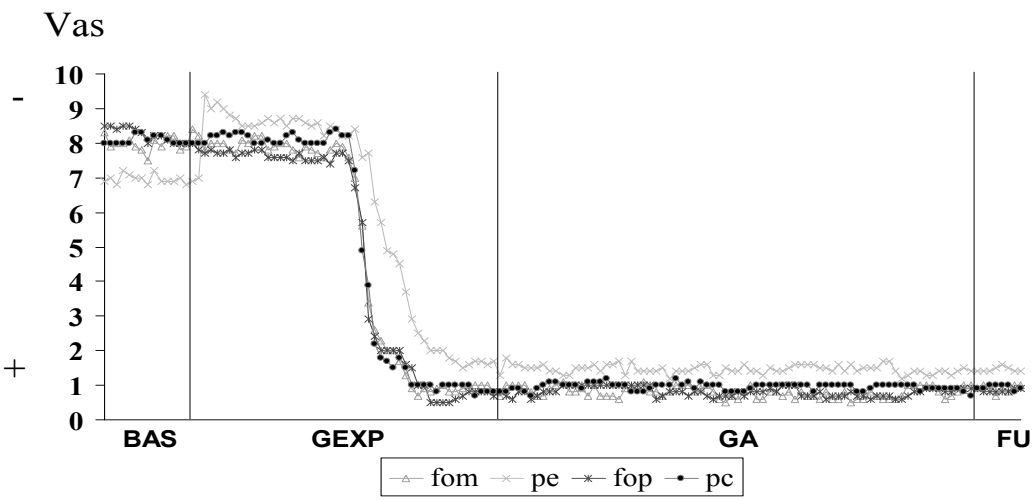

\section{Condition II (Baseline-GA-GEXP-FU)}

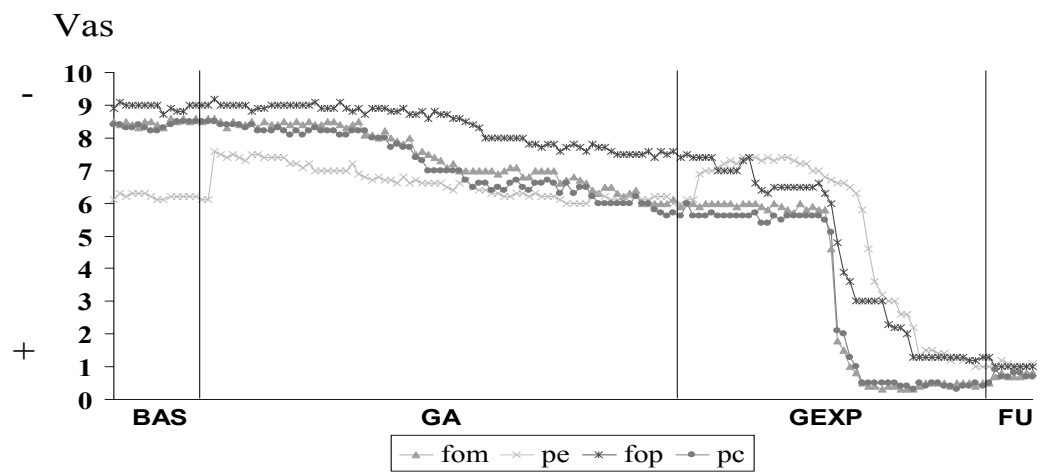

Figure 1 Means calculated from the time series of each patient within condition I $(n=4)$ and condition II $(n=4)$ for fear of movement/(re)injury (fom), pain experience (pe), fear of pain (fop), and pain catastrophizing (pc) as measured with the daily diary, across baseline (BAS), graded exposure in vivo (GEXP), graded activity (GA), and 6-month follow-up period (FU). BAS = 14 days, GEXP $=42$ days (12 sessions of 1 hour) +7 days of activity monitor; $\mathrm{GA}=70$ days (20 sessions of 1 hour) +7 days of activity monitor, $\mathrm{FU}=$ 7 days. VAS, visual analog scale. 


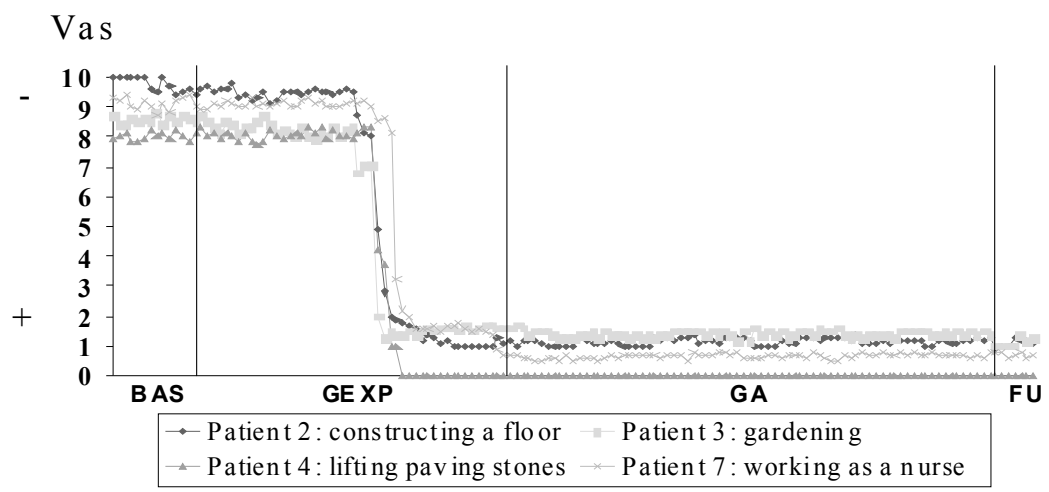

Condition II (B aseline-GA -GEXP-FU)

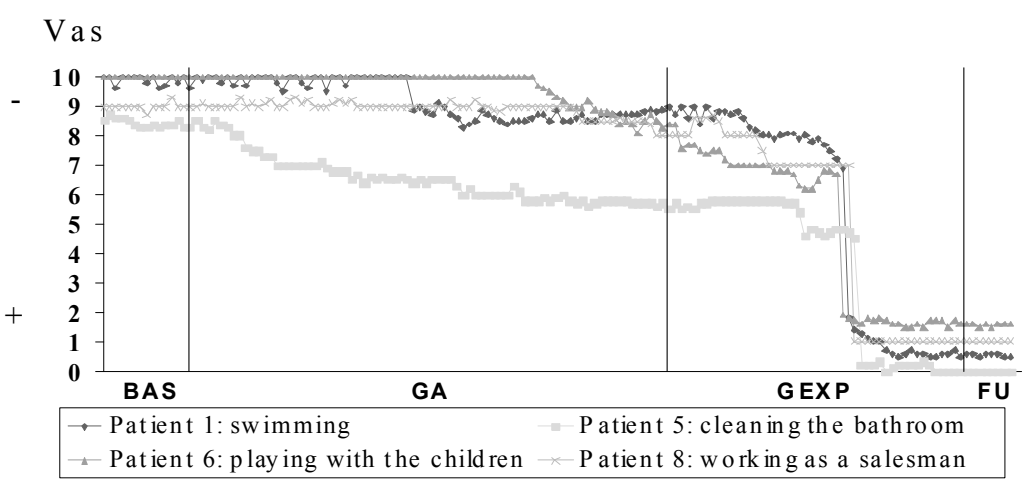

Figure 2 Mean daily measures for each patient in condition I and condition II for 1 of the 2 selected personally relevant activities, across baseline (BAS), graded exposure in vivo (GEXP), graded activity (GA), and 6-month follow-up period (FU). BAS = 14 days, GEXP $=42$ days (12 sessions of 1 hour) +7 days of activity monitor; $\mathrm{GA}=70$ days ( 20 sessions of 1 hour $)+7$ days of activity monitor, $\mathrm{FU}=7$ days. VAS, visual analog scale.

\section{Functional Disability}

Functional disability assessed by the NDI is shown in Table 2. In both conditions at the start and the end of BAS the mean score equates with "completely disabled." Clinically meaningful changes ( $\geq 5$-point change) are observed when GA (mean score of 35.5-27) as well as GEXP (mean score of 37.8-7.5) is introduced first. However, considering the disability categories for the NDI, patients in condition II are still "severely disabled" at the end of GA, whereas the patients in condition I report mild disability at the end of GEXP. When GEXP follows GA in condition II, the mean score for the NDI decreased further (mean score of 27-8.5), which means that the GEXP provides for a situation in which patients are "mildly disabled." The measurement 
periods after the GEXP did not show new clinical relevant changes in either category for functional disability.

Table 3 The Effect Lag During the Graded Exposure In Vivo in Which the Minimum $P$ Values for the Randomization Tests With 1 Observation Per Phase Has Been Reached for Each Patient in Condition I and Condition II for Fear of Movement, Pain Experience, and 2 Personally Relevant Activities

\begin{tabular}{lllll}
\hline Condition Interval & Fear of Movement & Pain Experience & Activity 1 & Activity 2 \\
\hline Condition I & & & & \\
Patient 2 & $5(P=.024)$ & $5(P=.029)$ & $5^{\mathrm{a}}(P=.024)$ & $5^{\mathrm{b}}(P=.024)$ \\
Patient 3 & $5(P=.024)$ & $5(P=.024)$ & $5^{\mathrm{c}}(P=.024)$ & $5^{\mathrm{d}}(P=.024)$ \\
Patient 4 & $5(P=.021)$ & $5(P=.024)$ & $5^{\mathrm{e}}(P=.021)$ & $5^{\mathrm{f}}(P=.021)$ \\
Patient 7 & $5(P=.029)$ & $5(P=.037)$ & $5^{\mathrm{g}}(P=.029)$ & $5^{\mathrm{h}}(P=.029)$ \\
Condition II & & & & \\
Patient 1 & $5(P=.013)$ & $5(P=.013)$ & $5^{\mathrm{i}}(P=.013)$ & $5^{\mathrm{j}}(P=.016)$ \\
Patient 5 & $5(P=.016)$ & $5(P=.024)$ & $5^{\mathrm{k}}(P=.016)$ & $5^{\mathrm{l}}(P=.016)$ \\
Patient 6 & $4(P=.024)$ & $5(P=.024)$ & $5^{\mathrm{m}}(P=.024)$ & $5^{\mathrm{n}}(P=.024)$ \\
Patient 8 & $4(P=.029)$ & $5(P=.037)$ & $5^{\circ}(P=.029)$ & $5^{\mathrm{p}}(P=.024)$ \\
\hline
\end{tabular}

NOTE. The effect lag ( 1 lag is 1 week or 2 sessions of exposure therapy) during the graded exposure in vivo in which the minimum $P$ values for the randomization tests with 1 observation per phase has been reached for each patient in condition I (baseline - graded exposure in vivo - graded activity - 6-month follow-up) and condition II (baseline - graded activity - graded exposure in vivo - 6-month follow-up) for fear of movement, pain experience, and 2 personally relevant activities. ${ }^{a}$ Mountain bike; ${ }^{b}$ construct a floor; ${ }^{\mathrm{c}}$ garden; ${ }^{\mathrm{d}}$ jogging; ${ }^{\mathrm{e}}$ lift paving stones; ${ }^{\mathrm{f}}$ play soccer; ${ }^{\mathrm{g}}$ ride; ${ }^{\mathrm{h}}$ work as nurse; ${ }^{\mathrm{i}}$ swim; ${ }^{\mathrm{j}}$ look back; ${ }^{k}$ clean the bathroom; ${ }^{\prime}$ cycle; ${ }^{m}$ dance; ${ }^{n}$ having a romp with the children; ${ }^{\circ}$ drive a car; ${ }^{p}$ work as salesman.

\section{Pain-Related Fear}

Clinically relevant change for pain-related fear, defined by a minimum of $50 \%$ decrease on the mean TSK score, is only observed when GEXP is delivered (Table 2). In condition I the mean TSK score decreased from 47.5 at the start of GEXP to a mean TSK score of 24 at the end of GEXP. This clinically relevant reduction remains after GA and at follow-up. In condition II, there is a slight decrease at first of the mean TSK score from 48 to 41 as the result of GA and a clinically relevant change to 23 at the end of GEXP, which remains at follow-up.

\section{Physical Activity}

The physical activity data obtained by accelerometry are summarized in Table 4. Compared with BAS, in condition I a marked increase of physical activity, expressed as total movement counts per minute of days registered, is observed during GEXP ( $220 \pm 8.75$ to $574 \pm 17.75)$. When GA followed BAS, as prescribed for condition II, physical activity was also increased (217 \pm 7.98 to $356 \pm 6.09)$ but to a lesser extent than in condition I in which GEXP followed BAS. In condition II, physical activity increased further when GEXP followed GA (356 \pm 6.09 to $564 \pm 23.30)$. In contrast, the degree of physical activity did not change appreciably when GA follows GEXP (condi- 
tion I). Regarding physical activity during follow-up in condition I as compared with $\mathrm{GA}$, a slight reduction is observed ( $578 \pm 22.43$ to $543 \pm 12.69)$, whereas in condition II, the degree of physical activity remains almost the same as at the end of GEXP (564 \pm 23.30 to $561 \pm 22.91$ ). However, in both conditions, physical activity at follow-up is much higher than at BAS.

Table 4 Total Amount of Physical Activity, Adjusted for Time Wearing the Activity Monitor in Condition I and Condition II

\begin{tabular}{|c|c|c|c|c|}
\hline \multirow[b]{2}{*}{ Condition Interval } & \multicolumn{4}{|c|}{ Total Physical Activity (counts $\cdot \mathrm{min}^{-1} \cdot \mathrm{d}^{-1}$ ) } \\
\hline & Mean & Minimum & Maximum & SD \\
\hline \multicolumn{5}{|l|}{ Condition I $(n=4)$} \\
\hline Baseline & 220 & 208 & 232 & 8.75 \\
\hline GEXP & 574 & 554 & 601 & 17.75 \\
\hline GA & 578 & 548 & 611 & 22.43 \\
\hline 6-mo follow-up & 543 & 529 & 564 & 12.69 \\
\hline \multicolumn{5}{|l|}{ Condition II $(n=4)$} \\
\hline Baseline & 217 & 210 & 229 & 7.98 \\
\hline GA & 356 & 348 & 364 & 6.09 \\
\hline GEXP & 564 & 545 & 602 & 23.30 \\
\hline 6-mo follow-up & 561 & 543 & 598 & 22.91 \\
\hline
\end{tabular}

Abbreviations: GEXP, graded exposure in vivo; GA, graded activity.

NOTE. Total amount of physical activity (expressed as activity counts per minute of days registered; counts $\cdot \mathrm{min}^{-1} \cdot \mathrm{d}^{-1}$ ) adjusted for time wearing the activity monitor in condition I (baseline - graded exposure in vivo - graded activity - 6-month follow-up) and condition II (baseline - graded activity - graded exposure in vivo-6-month follow-up).

\section{Discussion}

This is the first study showing that the effects of GEXP generalized to patients with chronic PTNP reporting elevated levels of pain-related fear. By using a replicated single-case, crossover, experimental phase design, the aim of this study was to examine the effectiveness of GEXP as compared with GA in reducing the threat value of physical activities and/or movements and to restore daily functioning in 8 patients with chronic PTNP reporting substantial fear of movement and/or (re)injury. The patients were referred for outpatient behavioral rehabilitation. Both the randomization tests on the daily measures and the pre- and post-treatment phase measures showed that compared with GA, GEXP was superior in decreasing levels of pain-related fear, pain catastrophizing, pain disability, and pain experience, both after treatment and at follow-up. However, it should be mentioned that the results of the nondaily measures, with limited number of data points, were not subjected to randomization tests and for the most part based on arbitrary preset criteria. Improvements were found not only in the self-report measures but also in physical activity in the home situation, as measured with ambulatory activity moni- 
tors. Because the experimental design did not include washout periods between the different treatment components, it is likely that carry-over effects occurred. Indeed, when GA followed GEXP, the improvements remained stable, which is also consistent with the favorable follow-up results.

As noted in the introduction, GA showed to be helpful for chronic neck pain disability across a number of studies. However, in the current study, change during GA was marginal at best when GA preceded GEXP. Both GA and GEXP were performed according to a specific protocol. In addition, a different therapist team gave GA and GEXP. Because patients' attitudes and beliefs, and thereby patients' disability levels, may be derived from the projected attitudes and beliefs of health care providers (Rainville et al., 1995), the 2 teams were comparable in terms of experience and therapists' preferences. Efforts were made to achieve the same level of enthusiasm in each therapist who participated in either GA or GEXP.

Besides the superiority of GEXP over GA, there is a sudden and remarkable level of improvement around sessions 7 to 9 during GEXP. The content of these sessions mainly consisted of exposure to personally relevant activities that represented the main functional goals that were chosen by the patients themselves. Research into return of fear and contextual renewal shows that the beneficial effects of exposure are more or less confined to the context in which the exposure treatment was performed (Bouton, 2002; Rachman \& Whittal, 1989; Vansteenwegen et al., 2007). This means that confrontations with the (previously) fearful activity in other contexts could elicit a certain level of fear. However, the results in this study show that overall daily activity, as measured by the activity monitor, increased both in the short and long term. It seems likely that daily life activities not only consisted of situations that were part of GEXP.

GEXP has successfully been applied to patients with CLBP. Besides some single-case, experimental studies, randomized controlled clinical trials also have shown positive results recently with regard to pain disability and pain-related fear (Boersma et al., 2004; De Jong et al., 2005; Leeuw et al., 2008; Linton et al., 2008; Vlaeyen et al., 2001; 2002a; 2002b; Woods \& Asmundson, 2008). However, in comparison with the single-case, experimental exposure studies in patients with low back pain (De Jong et al., 2005; Vlaeyen et al., 2001; 2002a; 2002b), the current study shows that more exposure sessions are needed to demonstrate trend changes and significant effects in patients with PTNP. A possible explanation is that neck pain patients experience multiple complaints and fears, not just fear of movement. Besides neck pain, symptoms such as headache, visual disturbances, dizziness, weakness, paraesthesia, nausea, both upper and lower limb numbness and tingling, tinnitus, and cognitive problems (concentration and memory disturbances) are common in the acute stage after a traumatic event (Barnsley et al., 1994; Ferrari et al., 2005). In CLBP, patients' 
main concern is overall the experienced pain interfering with daily life activities. In addition, the concerns of the neck pain patients may be more difficult to challenge (eg "If I would lift heavy weights, then I do not have full control of my neck, which will worsen the pain complaints, with the result that I will not be able to do my job in the future").

It is of interest that current pain experience was also affected by GEXP. Moreover, the current results suggest that the decrease in pain experience temporarily follows an associated decrease in pain-related fear. Similar results are observed in the single-case, experimental studies of GEXP in CLBP and are in line with the fearavoidance model (De Jong et al., 2005; Vlaeyen et al., 2001; 2002a; 2002b). However, such strong reduction in pain is not common in usual cognitive-behavioral treatments for chronic pain (Kole-Snijders et al., 1999; Morley et al., 1999). How can this unexpected result be explained? One explanation is that fear reduction is associated with a decrease in muscle activation (Nederhand et al., 2006), which in turn may be associated with a reduction of pain experience (Flor et al., 1985). By avoiding the use of painful muscles to prevent the amplification of pain and further injury, muscle activation is decreased. Alternatively, experimental studies on the role of attention and pain-related fear have shown that patients with elevated levels of pain-related fear habitually attend to somatic sensations (Asmundson et al., 1997; Peters et al., 2002). This finding corroborates the idea that the most important function of anxiety is the early detection of potentially threatening situations. It is likely that the decrease in pain experience during GEXP was mediated by a process in which the reduction of the threat value of previously fear-eliciting stimuli also produced a redirection of the attention away from pain and bodily sensations. Finally, pain reduction might be the direct result of the diminished threat value of physical activities (Arntz \& Claassens, 2004). This is in line with recent imaging studies showing a relationship between catastrophizing and activity in cortical regions associated with affective, attention, and motor aspects of pain (Seminowicz \& Davis, 2006).

Despite the overall positive influence of GEXP on pain experience, there appears to be an increase in pain experience at the onset of GEXP. A possible explanation may have to do with the natural history of the participants. They could all be characterized as not-at-fault drivers. Research has shown that the not-at-fault driver is angry at someone else's actions (Ferrari et al., 2005; Mayou, 1994). They interact with the other driver and others with a notion of that "stupid driver" injured them and keeping him/her from attaining an important goal (Berkowitz \& Harmon-Jones, 2004; Ferrari et al., 2005). Besides, also subjectively aversive conditions which are the result of the injury can generate anger (Berkowitz \& Harmon-Jones, 2004). An example of such aversive conditions could be exposure to activities and/or move- 
ments in which physical discomfort or pain will be experienced (Anderson et al., 1998; Berkowitz \& Harmon-Jones, 2004). The induction of anger, for his part, and also pain-evoked cardiac responses that are modulated by anger produces increased pain intensity and pain unpleasantness (Rainville et al., 2005).

Finally, several limitations of the study should be mentioned. First, this study is limited in that it included only 8 patients. However, a replicated crossover, singlecase, experimental design was chosen with a customized randomization test to perform statistical analyses, which is an added value to detect idiosyncratic functional relationships and behavioral laws. Second, because in the crossover design all patients received both GEXP and GA, long-term differential effects could not be established. Replication studies in the form of randomized, controlled trials using larger samples are warranted. However, single-case experiments have higher practicality as compared with randomized, controlled trials and therefore are more useful to demonstrate accountability in a clinical setting on a more regular basis (Persons \& Silberschatz, 1998). In addition, the application of single-case experiments is an obvious option if the research interest is in the evaluation of individualized custommade therapy (Onghena \& Edgington, 2005). Third, by definition, it is not possible within single case studies to assess generality across subjects. However, interventions that produce dramatic effects are likely to generalize more than those with weaker effects, and this appears to be true in this study. Using randomization tests as time series analysis, we have demonstrated that the changes could not be attributed to chance. Besides, generalization may be derived from the fact that replications of eight different patients show consistently similar results in this study and in studies of patients with CLBP. So far, it seems justifiable to generalize the results to other patients with chronic PTNP who report substantial pain-related fear. However, it should be mentioned that patients who also report serious psychopathology did not participate in this study. Regarding limits of using GEXP to treat patients with fear or anxiety and significant psychopathology, the literature is not univocal. The same goes for the effect of psychopathology in cognitive-behavioral interventions as treatment for chronic pain. Therefore, it is quite possible that GEXP may also be a successful intervention for patients with PTNP who report serious psychopathology. In this study, the decision about excluding patients with serious psychopathology was based on criteria of earlier trials (Kole-Snijders et al., 1999; Smeets et al., 2006b; Hout van den et al., 2003). Fourth, this study did not check whether pain behavior has decreased as a result of GA. Finally, the follow-up period may not have been sufficient to determine the long-term effect on the treatment or long-term disability.

In sum, the current study supports a GEXP approach in chronic PTNP patients reporting substantial levels of pain-related fear. The GEXP was successful in decreasing 
levels of self-reported pain-related fear, disability, pain experience, and increasing the level of daily life physical activity as measured with an accelerometry-based activity monitor. These results underscore the idea that GEXP modifies the meaning people attach to their neck pain complaints, and those changes also influence the experienced painfulness. The results need to be verified in a wider chronic PTNP population. However, providing patients who report pain-related fear with a structured exposure in vivo program seems a promising treatment direction.

Supported by grant No. 940-31-071 of the Netherlands Organisation for Health Research and Development (ZonMw).

\section{Acknowledgements}

The authors thank Marlies den Hollander, Christoph Loo, Corine Cuypers, Marianne Maas, and Joop Ruijgrok, of the Department of Occupational Therapy, Physiotherapy, and Rehabilitation, University Hospital Maastricht, the Netherlands, and Mario Geilen, Herman Mulder, Noël Dortu, and Peter Heuts, of Hoensbroek Rehabilitation Center, Heerlen, The Netherlands, for their therapeutic input during the study. 



\section{Chapter 4}

\section{Reduction of pain-related fear in Complex Regional Pain Syndrome type I: The application of Graded Exposure in Vivo}




\begin{abstract}
Fear of (re)injury/movement has been identified as a potential predictor of chronic disability in complex regional pain syndrome type I (CRPS-I). In order to reduce painrelated fears and pain disability, graded exposure in vivo (GEXP) is likely to be an appropriate treatment. Indeed, there is evidence that in chronic pain patients reporting substantial fear of (re)injury/movement, GEXP is successful in reducing pain disability. However, the efficacy of exposure-based protocols in the treatment of CRPS-I patients for reducing pain disability has not been tested. The main research question of this study was whether the reduction of pain-related fear through GEXP also resulted in a decrease of disability in a subgroup of patients with CRPS-I who report substantial pain-related fear. A single-case experimental ABCD-design was used with random determination of the start of the intervention. Eight patients with CRPS-I were included in the study. To assess daily changes in pain intensity, painrelated fear, pain catastrophizing, and activity goal achievement, a diary was used. Standardized questionnaires of pain-related fear, pain disability, and self-reported signs and symptoms of CRPS-I were administered before and after each intervention, and at 6-month follow-up. The current study supports a GEXP approach to chronic CRPS-I. The GEXP was successful in decreasing levels of self-reported painrelated fear, pain intensity, disability, and physiological signs and symptoms. These results support the hypothesis that the meaning people attach to a noxious stimulus influences its experienced painfulness, and that GEXP activates cortical networks and reconciles motor output and sensory feedback.
\end{abstract}




\section{Introduction}

Complex regional pain syndrome type I (CRPS-I) is a poorly understood chronic pain disorder with regard to pathophysiology and treatment. A limited number of studies have focused on the psychological factors that may modulate the development of chronic disability in CRPS-I. One factor that has been suggested as a potential predictor of disability is fear of injury, as patients with CRPS-I are often unaware that their pain condition resulted from a minor trivial event (Nelson, 2002). This fear, combined with the increased pain sensitivity may lead to excessive guarding and overprotective behaviors that may worsen the pain problem. Indeed, there is evidence that in other musculoskeletal pain disorders such as back and neck pain, painrelated fear and associated avoidance behaviors contribute to the development of chronic pain disability (Keefe et al., 2004; Linton, 2000b; Nederhand et al., 2004; Vlaeyen \& Linton, 2000).

In the process of customization of cognitive behavioral treatments of excessive fears and anxiety, exposure to the feared stimuli appeared to be the most essential component. In order to reduce fear, the bio-informational theory of fear (Lang et al., 1998) predicts that first the fear network needs to be activated, and second, new information needs to be available that disconfirms the fear expectations that are inherent to the fear memory. On basis of these ideas and experimental support (Crombez et al., 1996; Goubert et al., 2002; Rachman, 1994), Boersma et al. (2004), De Jong et al. (2005) and Vlaeyen et al. (2001, 2002a) conducted a number of studies to examine the effectiveness of graded exposure in vivo in reducing painrelated fears and pain disability in chronic low back pain patients reporting substantial fear of movement/(re)injury. The remarkable improvements that are observed whenever graded exposure was initiated seem to suggest that the therapeutic power of exposure is very strong in the treatment of pain-related fear. Not only were improvements found on measures of pain-related fear and pain disability, but they were also generalized to increases in physical activity in the home situation.

For the treatment of CRPS-I a few evidence-based treatment regimens are available so far. A limited number of treatments focusing on physiotherapeutic algorithm and modalities (Egle and Hoffmann, 1990; Geertzen et al., 1994; Oerlemans et al., 1999; Richlin et al., 1978; Schwartzman \& Kerrigan, 1990) as well as cognitive-behavioral treatment (Geertzen et al., 1994; Lee et al., 2002) also have shown to be effective in patients with CRPS-I. However, the efficacy of exposure-based protocols in the treatment of CRPS-patients for reducing pain-related fear and pain disability has not been tested (Harden, 2005; Keefe et al., 2004). 
Using a replicated single case experimental design, we decided to experimentally examine whether the validity of graded exposure in vivo extends to patients with CRPS-I. The main research question of this study was whether the reduction of painrelated fear through graded exposure in vivo also resulted in a decrease of disability in a subgroup of patients with CRPS-I who report substantial pain-related fear. Besides, the contribution of a psycho-educational session, as part of the exposure, was examined.

\section{Materials and methods}

\section{Study design}

A single-case experimental $A B C D$-design was used with random determination of the start of the intervention (see also Onghena \& Edgington, 2005). Measurement period $A$ was a baseline (BAS) during which no new treatments where started. After BAS all patients received an education (EDU), followed again by a no-treatment period (B). Thereafter, the graded exposure in vivo (GEXP) was delivered (C). Finally, at 6-month there was a follow-up period of 1 week (D). To examine whether EDU, as particular component of GEXP, is effective in decreasing pain-related fear and/or disability, the $A B C D$-design uses control variables to demonstrate the effect of both interventions. These control variables are BAS and the no-treatment period after EDU. Experimental control is demonstrated if the changes in the patients' behavior and beliefs follow the changes in treatment conditions. Besides, by varying the length of each intervention phase, evident changes in treatment conditions are less the result of coincidence (Morley, 1996). In this study, two randomizations occurred.

First, the initiation of EDU was randomly assigned to the 8th or 15th day of the experiment. After that, the duration of the EDU period was also randomly assigned to 7 or 14 days. In this design, there were four possible assignments: $A$ ( 7 days)-B (7 days) - C, or A (7 days)-B (14 days)-C, or A (14 days)-B (7 days)-C, and A (14 days)-B (14 days) $-C$. Two kinds of outcome measures were included. To assess daily changes in pain intensity, pain-related fear, pain catastrophizing, and activity goal achievement, a diary was used. Standardized questionnaires of pain-related fear, pain disability, and self-reported signs and symptoms of CRPS-I were administered before and after each intervention, and at 6-month follow-up.

\section{Participants}

Eight patients with CRPS-I were included in the study. The sample was all female $(100 \%)$, with a mean age of $40 \pm 10.20$ (SD) years. In two patients CRPS-I was located 
in the right lower extremity, one patient in the left lower extremity, four patients in the right upper extremity, and one patient in the left upper extremity. In six patients, CRPS-I occurred on the dominant side. At inclusion, the mean duration of the complaints was $3.0 \pm 1.11$ years. The diagnosis of CRPS-I was made according to the criteria formulated by the IASP, and based on physician evaluation of objective symptoms. In order to select a subgroup of patients that also met the modifications that have been suggested to these criteria, the stringent criteria as formulated by Bruehl et al. (1999) and Veldman et al. (1993) were also assessed: altered skin color, altered skin temperature, edema (swelling), reduced range of motion, trophic (hair, nail skin). Other inclusion criteria were that the patients reported substantial fear of movement/(re)injury (Tampa Scale for Kinesiophobia (TSK: Miller et al., 1991) score >39), pain for at least 6 months and age between 18 and 65. The cut-off is based on the median of the TSK distribution of chronic low back pain samples reported earlier (Crombez et al., 1999; Vlaeyen \& Linton, 2000). Exclusion criteria were illiteracy, pregnancy, impairment of contra lateral extremity (e.g. because of rheumatoid arthritis, prior sympathectomy of the affected extremity), alcohol or drug abuse, and serious psychopathology based on the Symptom Checklist of which Dutch norms are available.

\section{Procedure and program overview}

Patients referred to the department of Pain Management and Research, and the department of Rehabilitation of the University Hospital Maastricht or the Hoensbroeck Rehabilitation Center who met the selection criteria, and who gave their written informed consent, were subsequently invited for an semi-structured interview, during which a cognitive and behavioral analysis of their pain problem was made.

A fear hierarchy was established by means of the Photograph series of Daily activities for the upper extremities (PHODA-UE: Dubbers \& Vikström, 2003) or the lower extremities (PHODA-LE: Jelinek et al., 2003). After this assessment, patients started with the BAS, during which they completed daily measurements at home. This period was followed by the exposure in vivo, which consisted of two steps. In the first step the EDU was given, followed again by a no-treatment period. After that, the GEXP started and was spread over 10 weeks and consisted of 20 sessions of $1 \mathrm{~h}$ each.

The interventions were carried out by a behavioral therapist, who is experienced in the field of pain, supervised by a rehabilitation specialist.

\section{Education (EDU)}

Patients were given a careful explanation of the fear-avoidance model, using the patient's individual symptoms, beliefs and behaviors to illustrate how vicious circles 
(pain>catastrophic thoughts $>$ fear $>$ avoidance $>$ disability $>$ pain) maintain the pain problem. The main goal of this part was to unambiguously educate the patient in a way that the patient views the various autonomic and vasomotor disturbances as a condition that can be self-managed, rather than a disease that needs careful protection. The aim was to correct the misinterpretations and misconceptions that have occurred during the development of the pain-related fear. One of the major goals of this educational part was to increase the willingness of the patient to finally engage in activities, movements and situations he has been avoiding for a long time.

\section{Graded exposure in vivo (GEXP)}

The most essential step consisted of graded exposure to the situations the patients had identified as 'dangerous' or 'threatening'. Based on the graded hierarchy of fear-eliciting situations (PHODA), individually tailored practice tasks were developed. Further, the general principles for exposure were followed. The patient agreed to perform certain activities or movements or stay in situations that he or she had been avoiding. Patients were also encouraged to engage in these fearful activities, movements or situations as much as possible until anxiety levels had decreased. The therapist first modelled each activity or movement. Finally, the GEXP was presented as a start only, and the patient was encouraged to continue exposing him or herself to more activities in everyday life after termination of the treatment sessions. To facilitate independence and to promote generalization, the presence of the therapist was gradually withdrawn, and contexts were created that mimicked those of the home situation. A detailed description of the exposure in vivo treatment can be found in Vlaeyen et al. (2002c).

\section{Outcome measures}

\section{Functional disability}

For CRPS-I patients with upper extremity pain the Radboud Skills Questionnaire (RASQ: Oerlemans et al., 1999; 2000a) was used. The RASQ scored patient's ability to perform activities of daily living in the normal manner. Representative items in the domain 'disabilities due to a hand disease' were measured with questions divided into 11 categories, ranging from personal hygiene to meal preparation and recreational activities. All questions were scored on a numerical way (1-5, with an extra score of ' 9 ' for 'not applicable'). The questionnaire has been found reliable in CRPS-patients, with good agreement between the outcomes for the test-retest and the interobserver reliability studies as established with the method of Bland and Altman (1986). For patients with CRPS-I lower extremity pain, the Walking Stairs Questionnaire (WSQ: Roorda et al., 1996a; 2004) and Questionnaire Rising and Sitting Down (QRS: Roorda et al., 1996b) were used. The QRS consisted of two subscales. One subscale ('QRS high chair') focused on rising from an sitting down on 
high seats, including lavatories and beds, and the other ('QRS low chair') focused on rising from and sitting down on low seats, such as easy chairs, car seats, and sofas. The questionnaire consisted of statements, which could be answered affirmatively. For both subscales of the QRS, the scores were converted to fit into a 0-10 range. The WSQ was a self-administered questionnaire consisting of 15 items, formulated in behavioral terms. The items referedto what a patient actually does, and not to what a patient thinks that he/she could do. Dichotomous response options (YES box marked/YES box not marked) were chosen to facilitate interpretation. Sum scores ranged from 0 to 15 . In a group of patients living at home, with different lowerextremity disorders, Roorda et al. (2004) found a sum score median of 7. In terms of test-retest reliability as expressed by Spearman's $r$ (range 0.79-0.90 and 0.85-0.89 respectively) and the ICC (range $0.78-0.84$ and $0.84-0.87$ respectively) the WSQ and QRS were reliable (Perez et al., 2001; 2002).

\section{Fear of movement/(re)injury}

The Dutch version of the Tampa Scale for Kinesiophobia (TSK) was used. This questionnaire consisted of 17 items, measuring fear of (re)injury due to movement, scored on a 4-point scale. The TSK has been found reliable and valid (Roelofs et al., 2004, Vlaeyen et al., 1995a; 1995b) and also successfully been used in patients with CRPS-I (Nelson, 2002). In addition to the TSK, the Photograph series of Daily activities (PHODA) for upper and lower extremities was used. The PHODA was a standardized method during which patients were requested to judge the threat value of various physical movements from daily life activities represented by photographs. The patients were requested to place each photograph along a fear thermometer, consisting of a vertical line with 11 anchor points (ranging from 0 to 100) printed on a $60 \times 40 \mathrm{~cm}$ hardboard. The fear-thermometer was placed on a table in front of the patient. The instruction was: 'Please watch each photograph carefully, and try to imagine yourself performing the same movement. Place the photograph on the thermometer according to the extent to which you feel that this movement is harmful to your affected extremity (arm/leg)'. After completion of the test, each photograph is given a rating according to the position on the thermometer. A total score ranging from 0 to 100 is calculated as the sum of each rating, divided by 12.5 (the maximum total score). A similar version of the PHODA has been used successfully in previous studies (De Jong et al., 2005; Vlaeyen et al., 2001; Vlaeyen et al., 2002a; 2002b).

\section{Disease-related signs and symptoms}

Before and after each intervention, and at 6-month follow-up, self-reported signs and symptoms of CRPS-I where determined. Self-reported symptoms were hyperesthesia, temperature asymmetry, skin color symmetry, sweating alterations and edema. The patients measured them in a dichotomous way (present or absent). 
Diary

To check whether the GEXP indeed modified the fear appraisals, pain intensity, and activity goal achievement, a short questionnaire was used consisting of 14 visual analog scales (from 0 to 10). This diary was meant as manipulation check. The first 11 items represented main factors of existing questionnaires for pain-related fear, catastrophizing, and pain disability. These included the Dutch versions of the TSK, The Pain Anxiety Symptoms Scale (PASS: Larsen et al., 1997; McCracken et al., 1992; 1999), and the Pain Catastrophizing Scale (PCS: Crombez et al., 1996; Sullivan et al., 1995). Visual analogue scales (VAS) consisted of $10 \mathrm{~cm}$ horizontal lines followed the items derived from these questionnaires. These VAS's were anchored at the left and right by the words 'totally disagree' and 'totally agree'. Three main scores were derived, consisted of the mean scores (range 0-10) of the items from TSK, PASS and PCS, respectively. Besides daily measures of pain-related fear, pain intensity was measured daily with an additional visual analog scale anchored with 'no pain at all' at one extreme and 'worst pain experienced' at the other. The last two visual analog scales referred to the performance of personally relevant activities. Each scale consisted of the same question: 'How difficult was it to perform this activity today?' The scale was anchored with 'no problem at all' at one extreme and 'impossible' at the other. This measure was administered on a daily basis during the whole duration of the study and the follow-up period of 1 week. The patients were instructed to complete the scales each evening and to send the package the next day to the researchers. This measure has been shown to be sensitive to graded exposure in vivo in previous studies (De Jong et al., 2005; Vlaeyen et al., 2001; 2002a; 2002b).

\section{Treatment expectancy and credibility}

After the rationale, and before the actual exposure, patients completed one expectancy and one credibility item on visual analog scales, with 'not at all' and 'very much' on the extremes: 'Do you expect that the program will help you to cope better with your pain complaints?' (Expectancy), 'Do you believe that the treatment offered to you is a meaningful treatment for patients with CRPS-I?' (Credibility) (Borkovec \& Nau, 1972).

\section{Statistical analyses}

For analyzing the resulting data of the daily measures, a randomization test for single case experimental designs using the rationale of Edgington (1992) was carried out. Because graded exposure in vivo (C) was expected to be superior to baseline $A$, and the education (B), the null hypothesis that there is no differential effect for any of the measurement times was tested using a randomization test on the differences between $B$ and $A, C$ and $A, C$ and $B$. While the follow-up (D) was expected to be superior to $A$ and will not change in relation to $C$, differences between $D$ and $A, D$ 
and $\mathrm{C}$ were also tested using randomization tests. The analyses were performed using the SCRT software (Single-Case Randomization Tests, version 1.1; (Onghena \& Van Damme, 1994b)). The program also allowed the calculation of a combined Pvalue of each design when the cases were considered simultaneously in a metaanalysis according to Edgington's additive method. Finally, the test was repeated assuming delayed effects (effect lags of 2 weeks or four sessions of exposure therapy) until the minimal $P$-value $(P<005)$ was reached (for the general formula and algorithms see Edgington, 1992; Edgington, 1995; Onghena \& Edgington, 1994a; Wampold \& Furlong, 1981).

\section{Preset criteria for non-daily measures}

For PHODA, TSK, WSQ, QRS, and RASQ the limited number of data made it impossible to use randomization tests. Therefore, we decided to formulate pre-set criteria to conclude whether the treatment could be considered successful. For the TSK a reduction of more than 30 percentiles was considered relevant. In the absence of any norms for PHODA, we estimated that a $50 \%$ decrease would give us enough support that the threat value of the activities used in the GEXP had decreased. For the WSQ, QRS, and RASQ no norms were available. Because we hypothized that graded exposure in vivo would resulted in a decrease of disability, we estimated that a improvement of $50 \%$ on each subscale of these questionnaires would give support to this hypothesis. The physiological signs and symptoms were assessed on self-report, and were described in the results as such.

\section{Results}

\section{Internal validity checks}

\section{Expectancy and credibility check}

Expectancy (mean rating of $3.8 \pm 0.84$ SD on a visual analog scale from 0 to 10) and credibility (mean rating of $4.2 \pm 0.79 \mathrm{SD}$ ) ratings were relative low for the GEXP. This implies that the participants did not expected that the program would help to cope better with their pain complaints, and that they did not believed that the GEXP was a meaningful treatment for patients with CRPS-I.

\section{Daily measures}

Because the patterns of change for fear of movement/(re)injury, pain catastrophizing, and fear of pain were quite similar, figures 1-4 shows only the observations across time for fear of movement/(re)injury, pain intensity, and the performance of personally relevant activities for each patient. 
Patient 1

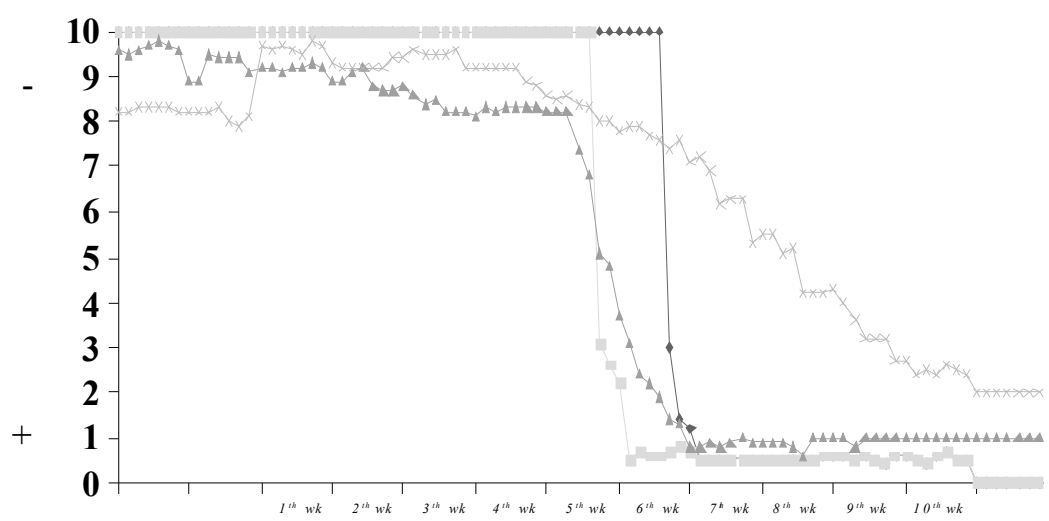

$\mathbf{A} \quad \mathbf{B} \quad \mathbf{C} \quad$ D

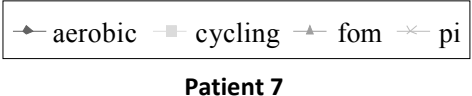

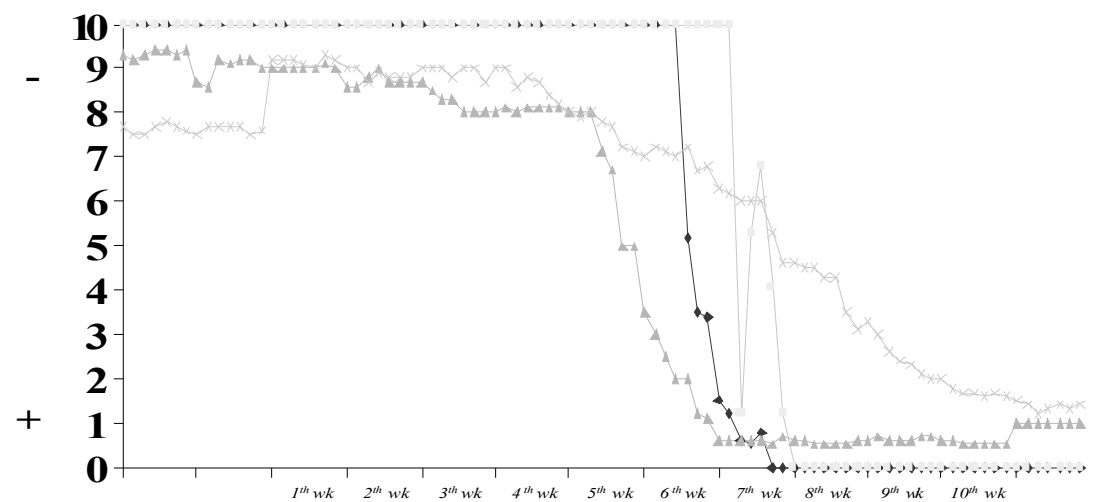



$\rightarrow$ push the baby buggy - left a child $\rightarrow$ fom $\div$ pi

\section{Figure 1}

Mean daily measures for patient 1 and 7 of fear of movement/(re)injury (fom), pain intensity (pi), and two personally relevant activities, across baseline (A), psycho-education (B), graded exposure in vivo (C), and 6-month follow-up period (D). $A=7$ days, $B=7$ days, $C=70$ days ( 20 sessions of $1 \mathrm{~h}$ ), $D=7$ days. 
Patient 5

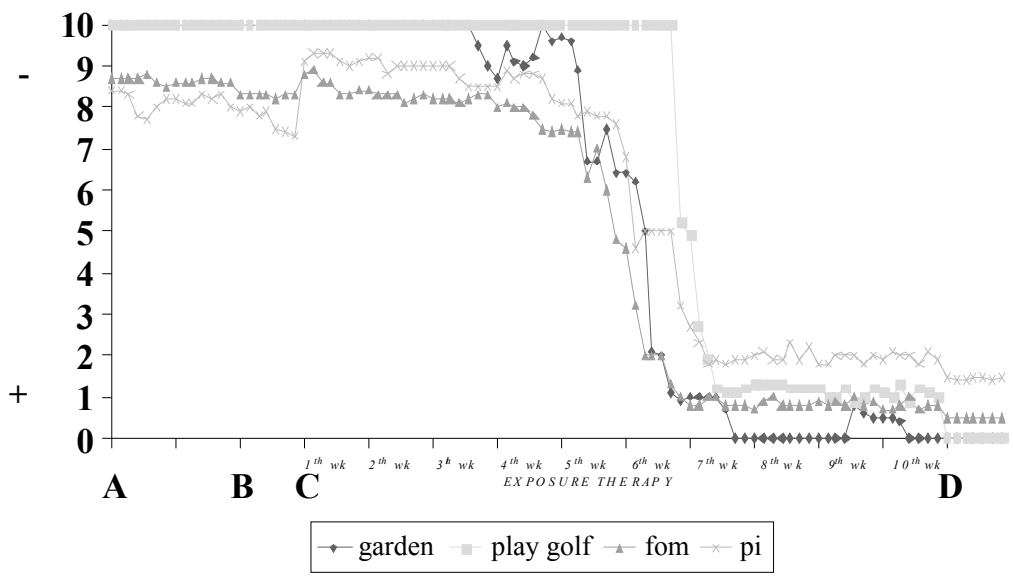

Patient 2

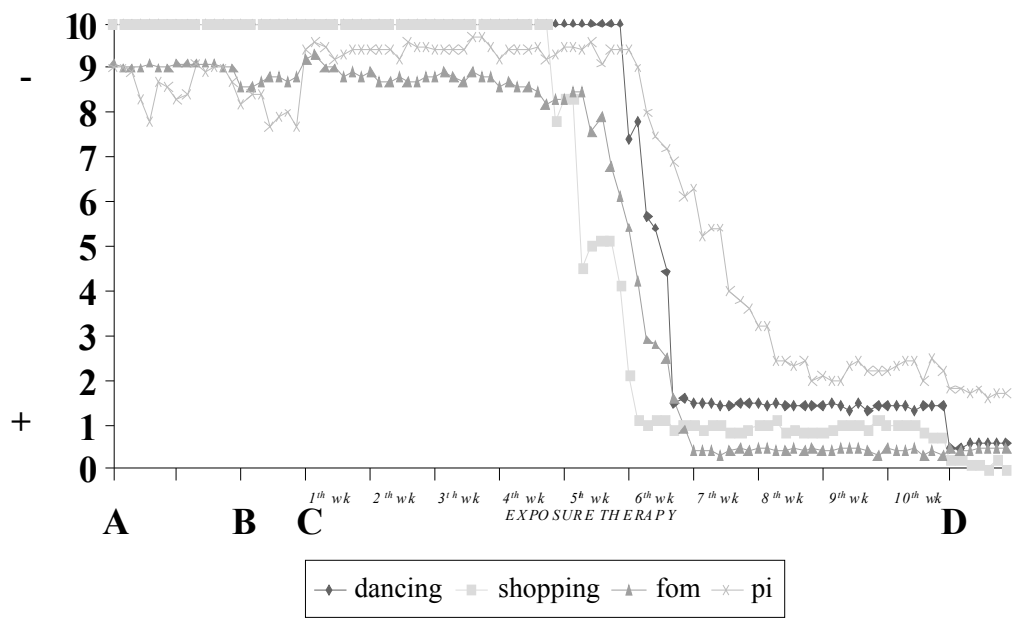

\section{Figure 2}

Mean daily measures for patient 5 and 2 of fear of movement/(re)injury (fom), pain, intensity (pi), and two personally relevant activities, across baseline (A), psycho-education (B), graded exposure in vivo (C), and 6-month follow-up period (D). $A=14$ days, $B=7$ days, $C=70$ days ( 20 sessions of $1 \mathrm{~h}$ ), $D=7$ days. 
Patient 3



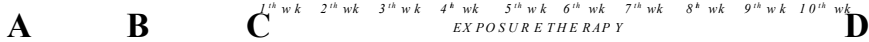

- play the piano - play the violin $\rightarrow$ fom $\leftarrow$ pi

Patient 6

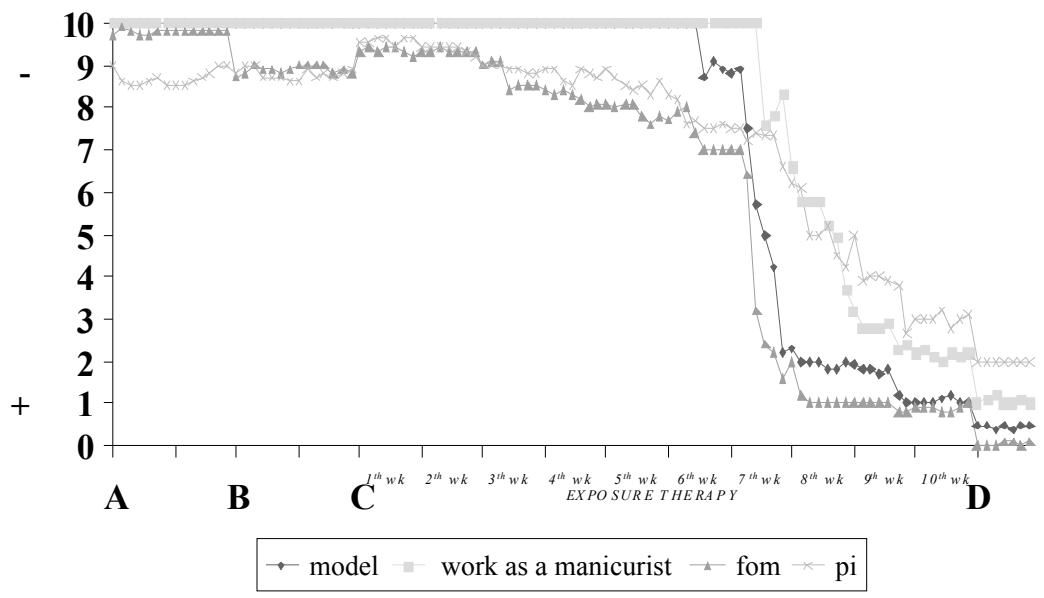

Figure 3 Mean daily measures for patients 3 and 6 of fear of movement/(re)injury (fom), pain intensity (pi), and two personally relevant activities, across baseline (A), psycho-education (B), graded exposure in vivo (C), and 6-month follow-up period (D). $A=14$ days, $B=14$ days, $C=70$ days (20 sessions of $1 \mathrm{~h}$ ), $D=7$ days. 
Patient 8

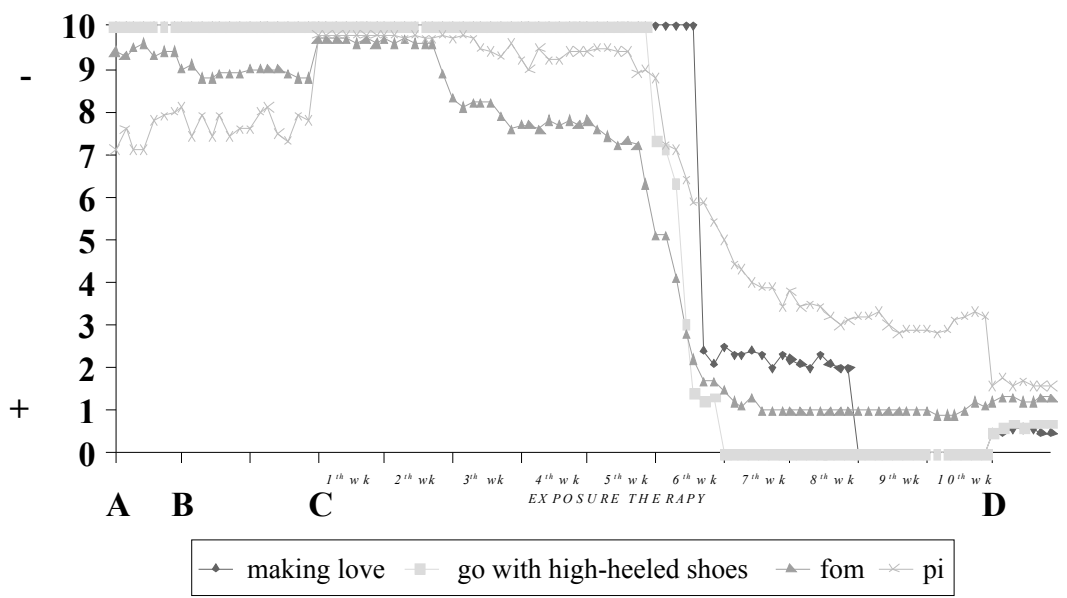

Patient 4

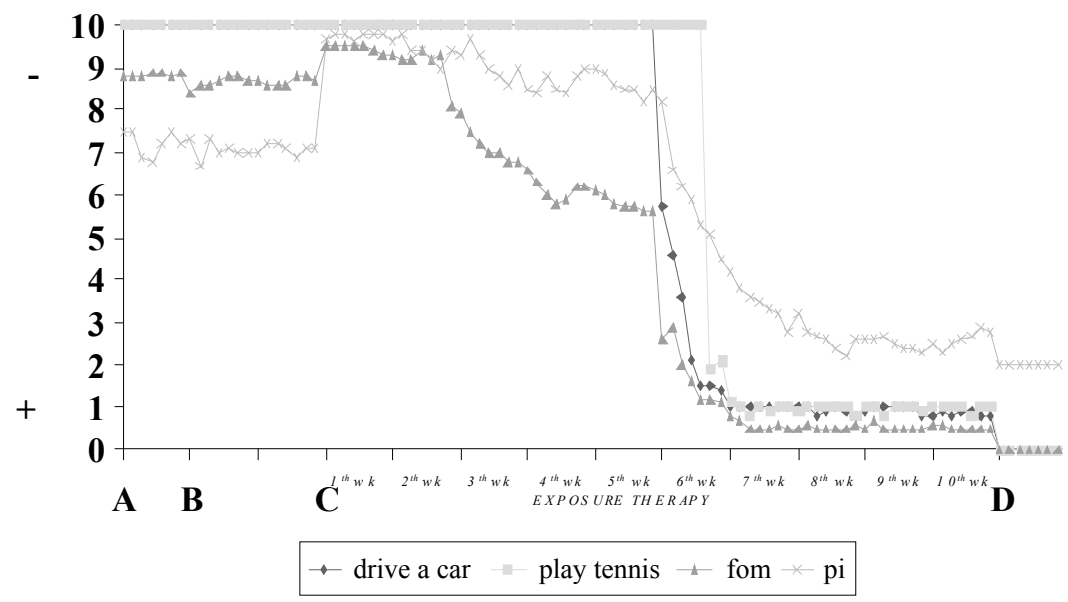

Figure 4 Mean daily measures for patients 8 and 4 of fear of movement/(re)injury (fom), pain intensity (pi), and two personally relevant activities, across baseline (A), psycho-education (B), graded exposure in vivo (C), and 6-month follow-up period (D). $A=7$ days, $B=14$ days, $C=70$ days (20 sessions of $1 \mathrm{~h}$ ), $D=7$ days.

Visual inspection reveals that the GEXP produced substantial trend changes, in fear of movement/(re)injury, pain intensity and the performance of personal relevant activities although these changes occurred during the second part of the GEXP. Table 1 displays the effect lag (one lag is 2 weeks or four sessions of exposure therapy) in which significance was reached for the phase design randomization tests on the raw data. The results appear to confirm the conclusions of the graphical display. For fear of movement significance was reached for patient 4 and 8 between week 3 
and 4 (sessions 5-8), for patient 1-3, 5 and 7 between week 5 and 6 (sessions 9-12) and for patient 6 between week 7 and 8 (sessions 13-16). With regard to pain intensity and personally relevant activities significance was reached either between week 5 and 6 (sessions 9-12) or between week 7 and 8 (sessions 13-16). When a combined P-value was calculated, the results for the eight P-values from table 1 of these four variables was in each case $P<0.0001$ (fear: $9.03 \times 10-11$; pain: $2.55 \times 10-10$; activity 1: $1.63 \times 10-10$; activity $2: 2.55 \times 10-10)$.

Table 1 The effect lag (one lag is 2 weeks or four sessions of exposure therapy) during the graded exposure in vivo in which the minimum $\mathrm{P}$-values for the randomization tests with one observation per phase has been reached for each patient for fear of movement, pain intensity, and two personally relevant activities

\begin{tabular}{|c|c|c|c|c|}
\hline Patient & Fear of movement/(re)injury & Pain intensity & Activity & Activity \\
\hline$\overline{1}$ & $3(P=0.029)$ & $3(P=0.029)$ & 4a $(P=0.029)$ & $3 b(P=0.029)$ \\
\hline 2 & $3(P=0.024)$ & $4(P=0.024)$ & $3 c(P=0.024)$ & $3 d(P=0.024)$ \\
\hline 3 & $3(P=0.024)$ & $4(P=0.037)$ & $3 e(P=0.024)$ & $4 f(P=0.037)$ \\
\hline 4 & $2(P=0.021)$ & $3(P=0.029)$ & $3 g(P=0.029)$ & $3 h(P=0.029)$ \\
\hline 5 & $3(P=0.024)$ & $3(P=0.024)$ & $3 i(P=0.024)$ & $3 j(P=0.024)$ \\
\hline 6 & $4(P=0.037)$ & $4(P=0.037)$ & $4 k(P=0.037)$ & $4 \mathrm{l}(P=0.037)$ \\
\hline 7 & $3(P=0.029)$ & $4(P=0.029)$ & $3 m(P=0.029)$ & $4 n(P=0.029)$ \\
\hline 8 & $2(P=0.021)$ & $3(P=0.029)$ & $30(P=0.029)$ & $3 p(P=0.029)$ \\
\hline
\end{tabular}

\section{Outcome measures}

\section{Functional disability}

In patients 1, 2 and 8 CRPS-I was located in the lower extremity. These patients reported also by the use of the WSQ and QRS about their functional disabilities. To permit mutual comparison of scales with different numbers of items, the scores of the WSQ and QRS were expressed in standardized scale sum scores, i.e. scores (range 0-10) were calculated as the proportion of the total possible score for the scale at issue multiplied by ten. The standardized sum scores of patient 1, 2 and 8, for each category of the WSQ and QRS, revealed an improvement of more than $50 \%$ after the GEXP and during FU (Table 2). In fact, all the three patients reported no functional disability after the GEXP and during FU. The scores of the FU did not change regard to the GEXP. Also the mean total score of the three subgroups for each category of the RASQ, calculated from patients 3-7, revealed an improvement of more than $50 \%$ (Table 3 ), except for the category 'work'. However, on closer inspection of the data it appeared that two patients answered 'not applicable' (score 9) for the question 'performing your occupation'. Before the pain complaints of 
these two patients started they were unemployed. Therefore, it could be that they had no comparison and where not able to imagine the situation.

Table 2 Standardised sum score from patient 1,2 and 8, for each catagory of the walking stairs questionnaire (WSQ) and questionnaire rising and sitting down (QRS) before and after each intervention and during follow-up

\begin{tabular}{|c|c|c|c|c|c|}
\hline Questionnaire & Patient & BAS & EDU & GEXP & FU \\
\hline \multicolumn{6}{|l|}{ WSQ } \\
\hline \multirow[t]{3}{*}{ Climbing stairs } & 1 & 0.63 & 0.63 & 0 & 0 \\
\hline & 2 & 3.13 & 3.13 & 0 & 0 \\
\hline & 8 & 5.00 & 5.00 & 0 & 0 \\
\hline \multirow[t]{3}{*}{ Walking in the house } & 1 & 6.50 & 6.50 & 0 & 0 \\
\hline & 2 & 6.50 & 6.50 & 0 & 0 \\
\hline & 8 & 6.50 & 6.50 & 0 & 0 \\
\hline \multirow[t]{3}{*}{ Walking outside } & 1 & 0.56 & 0.56 & 0 & 0 \\
\hline & 2 & 0.56 & 0.56 & 0 & 0 \\
\hline & 8 & 0.56 & 0.56 & 0 & 0 \\
\hline \multirow[t]{3}{*}{ Walking velocity } & 1 & 0 & 0 & 0 & 0 \\
\hline & 2 & 5.00 & 5.00 & 0 & 0 \\
\hline & 8 & 7.50 & 7.50 & 0 & 0 \\
\hline \multicolumn{6}{|l|}{ QRS } \\
\hline \multirow[t]{3}{*}{ High seat } & 1 & 9.09 & 9.09 & 0 & 0 \\
\hline & 2 & 7.73 & 7.73 & 0 & 0 \\
\hline & 8 & 8.18 & 8.18 & 0 & 0 \\
\hline \multirow[t]{3}{*}{ Low seat } & 1 & 0 & 0 & 0 & 0 \\
\hline & 2 & 5.29 & 5.29 & 0 & 0 \\
\hline & 8 & 9.41 & 9.41 & 0 & 0 \\
\hline
\end{tabular}

BAS, baseline; EDU, education; GEXP, graded exposure in vivo; FU, follow-up.

Table 3 Mean total score, calculated from patient 3-7, for each catagory of the radboud skills questionnaire (RASQ), determined at baseline, before and after each treatment module and during 6-month follow-up

\begin{tabular}{llcccc}
\hline Group & Catagory & BAS & EDU & GEXP & FU \\
\hline I. Personal care & 1 Personal hygiene & 25.2 & 25.2 & 8.4 & 8.4 \\
& 2 Toilet hygiene & 4 & 4 & 1.4 & 1.4 \\
& 3 Dressing & 26.2 & 26.2 & 9.8 & 9.8 \\
II. Domestic activities & 4 Eating and drinking & 4.4 & 4.4 & 1 & 1 \\
& 5 Housekeeping & 26.4 & 26.4 & 8.4 & 8.4 \\
& 6 Meal preparation & 35.2 & 35.2 & 13.2 & 13.2 \\
III. Other activities & 7 Taking care of clothes & 13.2 & 13.2 & 4.2 & 4.2 \\
& 8 Recreational activities & 10 & 10 & 2.8 & 2.8 \\
& 9 Social activities & 12.8 & 12.8 & 4.2 & 4.2 \\
& 10 Other items & 30.4 & 30.4 & 9.8 & 9.8 \\
& 11 Work & 6.6 & 6.6 & 4.4 & 4.4 \\
\hline
\end{tabular}

BAS, baseline; EDU, education; GEXP, graded exposure in vivo; FU, follow-up. 


\section{Fear of movement/(re)injury}

The mean TSK-score before and after each intervention was: BAS=54.75 ( \pm 5.18 SD), $\mathrm{EDU}=55.0( \pm 5.24 \mathrm{SD}), \mathrm{GEXP}=20.25$ ( $\pm 2.05 \mathrm{SD}), \mathrm{FU}=19.75$ ( $\pm 1.67 \mathrm{SD})$. For all patients a significant reduction was observed at the end of GEXP and during FU as compared to BAS and EDU. TSK scores decreased from a mean score of 55.0 (>80th percentile) to a mean score of 19.75 (<10th percentile). Similar results were found for PHODA: BAS=84.75 ( $\pm 3.96 \mathrm{SD}), \mathrm{EDU}=85.5$ ( $\pm 3.82 \mathrm{SD}), \mathrm{GEXP}=12.86( \pm 3.31 \mathrm{SD}), \mathrm{FU}=11.63$ ( $\pm 2.33 \mathrm{SD})$. Significant reductions were observed at the end of GEXP and during FU as compared to BAS and EDU (mean reduction is $90 \%$ ).

\section{Disease-related signs and symptoms}

Table 4 summarizes the frequencies of the self-reported CRPS-related signs and symptoms in the patients. These frequencies were expressed in percents of positive reports for CRPS-related signs and symptoms. Surprisingly, in every case, at the end of the GEXP all the patients reported a positive change for the better in CRPSrelated signs and symptoms. During FU there was even no report of any of the CRPS-related signs and symptoms. Despite the small sample, by using a nonparametric test (Wilcoxon test) the changes from BAS to GEXP ( $P=0.042)$ and BAS to FU $(P=0.039)$ were also significant.

Table 4 Self-reported signs and symptoms of CRPS (\% positive) by the experimental group across study periods

\begin{tabular}{lccc}
\hline CRPS-I characteristics & & & \\
Self-reported symptoms & BAS $(\mathbf{n}=\mathbf{8})$ & GEXP $(\mathbf{n}=\mathbf{8})$ & FU $(\mathbf{n}=\mathbf{8})$ \\
\hline Hyperesthesia & 100.0 & 0.0 & 0.0 \\
Edema & 87.5 & 0.0 & 0.0 \\
Skin color asymmetry & 87.5 & 25.0 & 0.0 \\
Temperature asymmetry & 87.5 & 25.0 & 0.0 \\
Sweating alterations & 50.0 & 0.0 & 0.0 \\
\hline
\end{tabular}

BAS, baseline; GEXP, graded exposure in vivo; FU, follow-up.

\section{Discussion}

This is the first study testing the effects of an exposure-based protocol in the treatment of CRPS-I patients for reducing pain-related fear and pain disability. The aim of this study was to experimentally examine whether the validity of the graded exposure in vivo extends to patients with CRPS-I. Eight patients with CRPS-I reporting substantial fear of movement/(re)injury, and who were referred for outpatient behavioral rehabilitation were included. A replicated single case experimental design was applied in which chronically disabled patients were assigned to EDU and GEXP. 
The randomization tests on the daily measures showed as well individually as in group that compared with a no-treatment baseline period and a psycho-educational session, a graded exposure in vivo treatment was successful in decreasing levels of pain-related fear, pain disability, and pain intensity. This last result is remarkable because pain intensity usually is not the target of the GEXP treatment. Similar are the results for the assessments at the start of BAS, before and after each intervention and during FU. There was an overall improvement in self-reported disability after GEXP, which was also reported during FU. Last but not least, the patients reported that the presence of signs and symptoms consistent with CRPS-I were significantly decreased after the GEXP and even disappeared at FU-assessment. This is remarkable in the context of the patients' average pain duration of 3 years without resolution.

In contrast to the fast changes in the daily measures during the graded exposure treatment of chronic low back pain patients (Vlaeyen et al., 2001; 2002a; 2002b; 2002c), in which the reduction of pain-related fear and disability was achieved within fewer than three exposure sessions, in CRPS-I these changes occurred in an later phase of the exposure treatment. An explanation of this delayed effect could be that the observed peripheral changes of CRPS-I mediated the effect of information on pain. This theory is supported by a study of Arntz and Claassens (2004). They tested whether meaning influences the experience of pain. By suggesting that a very cold metal bar was either hot or cold, the potentially tissue-damaging property of the stimulus was experimentally manipulated. The result of this experiment was that harm beliefs are a crucial aspect of meaning that influences the subjective intensity of pain. The long-term meaning (i.e. the autonomic consequences) of pain might be also play an important role. In low back pain patient's possible tissuedamage is not observable. For this reason, it might have been easier to modify the meaning attached to their pain as compared to the CRPS patients in whom the symptoms are clearly visible.

Additionally, these observable symptoms might be interpreted as tissue damage and may direct the attentional focus to the sensation, thereby amplifying the pain experience. There is indeed evidence that pain is experienced more strongly when the focus of attention is directed towards it (Arntz et al., 1991; 1994; Arntz \& de Jong, 1993; Janssen et al., 1998; Rode et al., 2001). In line with this is that a tissuedamage interpretation of pain might include anxiety, which in turn might amplify the experience of pain. Recent studies suggest that most of the influence of anxiety on pain is mediated by attention (Arntz et al., 1991; 1994; Arntz \& de Jong, 1993).

How can we explain the self-reported trophic changes or enhanced efficacy of nociceptive mechanisms or cortical changes by graded exposure in vivo? Disrupted 
body schema (Schwoebel et al., 2001) and shrinkage of the cortical representation of the effected limb in the primary somatosensory cortex (Juottonen et al., 2002) have both been observed in CRPS-I patients. Supposedly, common cortical mechanisms are thought to underlie post-stroke and post-trauma CRPS-I (Janig \& Baron, 2003; Riedl et al., 2001). Analogous to these findings it is notable that in phantom pain and in stroke patients, a primary goal is to activate cortical areas that subserve the affected limb, which leads to symptomatic and functional improvements (Flor et al., 2001; Liepert et al., 2000) and which in turn correlate with cortical reorganization (Flor et al., 2001; Kopp et al., 1999). It seems that graded exposure in vivo is a strategy that aims to activate cortical networks and might be successful for CRPS-I rehabilitation. It is not clear however, how the graded exposure in vivo might address either of these processes. A possible explanation might be that graded exposure in vivo provide for disruption of the internal body schema. This might raise the possibility of a guarding type mechanism that impacts higher order motor processes such as motor intent or motor planning. Similar approaches for phantom pain and acute CRPS-I are thought to reconcile motor output and sensory input (McCabe et al., 2003; Ramachandran, 1995), which implies that a mismatch between motor intent and sensory feedback is causative of pain. Galer and Jensen (1999) have demonstrated that some patients with CRPS-I have an involuntary neurological neglect-like condition.

Another explanation of the effect of graded exposure in vivo for CRPS-I might be that this treatment focused on mental and visual attention in order to move the affected extremity. Thus, perhaps the graded exposure in vivo requires the CRPS-I patient to attend, possibly at an involuntary level, to the affected extremity for a certain time. In which case, the graded exposure in vivo may simply serve to reverse a learned disuse of the affected extremity (Butler, 2001).

In addition to the exposure in vivo procedure, Moseley (2004) found that a motor imagery program (MIP) is also effective for CRPS-I in reducing pain and swelling and that this support the involvement of cortical abnormalities in the development of this disorder. The MIP is made up of 2 weeks each of hand laterality recognition, imagined hand movements with the effected hand and mirror therapy by which the unaffected hand is physical active. In contrast to the exposure in vivo procedure, the MIP did not involve physical movements and/or activities with the affected extremity. Although the mechanism of effect of the MIP are not clear and further research is needed, Moseley (2004) supposed that success is dependent on sequential activation of cortical pre-motor (by imaging) and motor (by mirror therapy) networks or sustained and focused attention to the affected limb. 
Finally, several limitations of this study should be mentioned. First, the preliminary results reported here are limited in that they are based on a small number of patients. However, a single-case experimental design was chosen with appropriate time series statistical analyses. Second, we did not compare the GEXP with other interventions. Replication studies in the form of randomized single-case crossover designs or randomized controlled trials using larger samples are warranted. Third, the study is impeded by the use of self-report measures only, which are subject to response bias. Our study merits replication in which self-report measures are supplemented with objective assessments of touch sensitivity, mechanical allodynia, skin temperature asymmetry, swelling and active range of motion. To test how CRPS-I patients respond in daily life situations, it would be interesting to use ambulatory activity monitors in every day life outside the clinic (Bussmann et al., 2001; Schasfoort et al., 2002; 2003). Finally, the follow-up period may not have been sufficient to determine the long-term effect of the treatment or long-term quality of life.

In sum, the current study supports a graded exposure in vivo approach in chronic CRPS-I patients reporting substantial levels of pain-related fear The GEXP was successful in decreasing levels of self-reported pain-related fear, pain intensity, disability, and self-reported peripheral abnormalities. These results underscore the idea that the GEXP modifies the meaning people attach to a their pain, and that these changes also influences the experienced painfulness. GEXP likely activates cortical networks and reconciles motor output and sensory feedback. The results need to be verified in a wider chronic CRPS-I population. However, the GEXP offer a promising treatment direction for what is a difficult pain disorder to treat. 

Chapter 5

\section{Fear of movement/(re) injury in chronic low back pain: Education or Exposure in Vivo as mediator to fear reduction?}

Jeroen R de Jong, Johan WS Vlaeyen, Patrick Onghena, Mariëlle EJB Goossens, Mario Geilen, Herman Mulder. Fear of movement/(re)injury in chronic low back pain: Education or exposure in vivo as mediator to fear reduction. Clin J Pain 2005; 21: 9-17. 


\begin{abstract}
Clinical research of graded exposure in vivo program (GEXP) in patients with chronic low back pain who reported fear of movement/(re)injury shows abrupt changes in self-reported pain-related fears and cognitions. The abrupt changes are more characteristics of insight learning rather than the usual gradual progression of trial and error learning. The educational session (EDU) at the start of GEXP might have contributed to this insight. The current study examines the contribution of GEXP versus an operant graded activity program (OPE) in the reduction of pain-related fear and associated disability and physical activity. Six consecutive patients with chronic low back pain who reported substantial fear of movement/(re)injury were included in the study. After a no-treatment baseline measurement period, all the patients received EDU, followed again by a no-treatment period. Patients were then randomly assigned to either GEXP or OPE. A diary was used to assess daily changes in pain intensity, pain-related fear, pain catastrophizing, and activity goal achievement. Standardized questionnaires of pain-related fear, pain vigilance, pain intensity, and pain disability were administered before and after each intervention and at the 6month follow-up. An activity monitor was carried at baseline, during the interventions, and 1 week at 6-month follow-up. Randomization tests of the daily measures showed that improvements in pain-related fear and catastrophizing occurred after EDU was introduced. The results also showed a further improvement when GEXP followed the no-treatment period after EDU and not during OPE. Performance of relevant daily activities, however, were not affected by EDU and improved significantly only in the GEXP condition. All improvements remained at half-year follow-up only in patients receiving GEXP. These patients also reported a significant decrease in pain intensity at follow-up.
\end{abstract}




\section{Introduction}

One of the recent developments in behavioral pain research concerns the mechanisms by which patients with acute pain become chronically disabled. A steadily increasing number of studies are showing that observable physical performance and self-reported disability levels in patients with sub acute and chronic pain are associated with cognitive and behavioral, rather than sensory and biomedical, aspects of pain (Asmundson et al., 1999; Linton, 2000b; Vlaeyen \& Linton, 2000). Fearavoidance models have been proposed, and their major assumptions are successfully tested (Lethem et al., 1983; Vlaeyen et al., 1995a; 1995b; Vlaeyen \& Linton, 2000; Waddell et al., 1993). A number of studies have reported that pain-related fear is one of the strongest predictors of variation in physical performance (AlObaidi et al., 2000; Burns et al., 2000; Crombez et al., 1996; 1999; Vlaeyen et al., 1995a). Prospective studies have revealed that, in patients with acute back pain, pain-related fear is predictive of future disability and work status (Fritz et al., 2001; Klenerman et al., 1995; Sieben et al., 2002). If pain-related fear is indeed one of important mechanisms responsible for the development and maintenance of chronic pain disability, fear reduction should enhance the prevention of further pain-related disability and distress.

Current treatments of excessive fears and anxiety are based on the experimental psychological work of Wolpe (1958) on desensitization. Later studies revealed that the exposure to the feared stimuli appeared to be the most essential component of the systematic desensitization. Craske and Rowe (1997) suggested that for fearful patients, first-hand evidence of actually experiencing themselves behaving differently is far more convincing than rational argument, and that the most essential step consists of graded exposure to the situations the patient has identified as dangerous or threatening. The bio informational theory of fear put forward by Lang et al. (1998) predicts that there are 2 main conditions to reduce fear. First, the fear network needs to be activated, and second, new information needs to be available that does not confirm the fear expectations that are inherent to the fear memory. Following from cognitive models such as the cognitive model of panic and the cognitive model of obsessions (Clark, 1986; Rachman, 1998), which assumes that cognitive errors can be corrected through conscious reasoning, behavioral experiments have been developed in which a collaborative empiricism is the bottom line. Exposure (situational and interoceptive) is introduced as a behavioral experiment in which catastrophic or irrational thoughts are being challenged.

Clinical support of exposure in vivo with behavioral experiments in chronic low back pain patients reporting substantial fear of movement/(re)injury has been reported by Vlaeyen et al. (Vlaeyen et al., 2001; 2002a; 2002b). Using a replicated single-case 
design, they provided preliminary evidence showing that compared with a notreatment baseline period and a Graded Activity program (based on operantbehavioral principles), the individually tailored exposure in vivo was superior in decreasing the self-report measures of pain-related cognitions and fears, pain control, and pain disability. Besides improvements on the self-report measures, an increase in physical activity in the home situation was also found with ambulatory activity monitors (Vlaeyen et al., 2002a). Although the exposure was provided during a period of 3 or 4 weeks, the reduction of pain-related fear was achieved within fewer than 3 exposure sessions of 1 hour. Such abrupt changes are more characteristic of insight learning rather than the usual gradual progression of trial and error learning (Rachman \& Whittal, 1989). In these studies, the presentation of the rationale at the start of the exposure might have contributed to this insight. Many patients reported that, for the first time, they received a credible rationale for their current level of disability.

If it seems that education has indeed a powerful treatment effect at the start of an exposure in vivo, there is the possibility of introducing treatment in a stepped care approach. To tease out the differential effects of the educational part and the actual exposure component found in the studies of Vlaeyen et al. (2001; 2002a; 2002b) we decided to: 1) examine whether an educational session alone reduces fear levels; and (2) to compare the effectiveness of education followed by graded exposure in vivo with behavioral experiments as compared with education with a operant graded activity program. By means of a replicated single-case experimental design, education (EDU) is contrasted with a graded exposure in vivo (GEXP) or operant graded activity program (OPE).

\section{Materials and methods}

\section{Study design}

A replicated single-case experimental design was used with multiple measurements. Patients were randomly assigned to treatment group $A B C$ or treatment group $A B D$. After a 3-week no-treatment baseline measurement period (A), all the patients received EDU, followed again by a 3-week no-treatment period (B). Then, random assignment to either 6-week GEXP (C) or 8-week OPE (D) occurred. Three kinds of outcome measures were included. To assess daily changes in pain intensity, painrelated fear, pain catastrophizing, and activity goal achievement, a diary was used. Standardized questionnaires of pain-related fear, pain vigilance, pain intensity, and pain disability were administered before and after each treatment, and at 6-month follow-up. To measure actual activity levels in the home situation, an activity moni- 
tor was carried at baseline, just after each intervention, and 1 week at 6-month follow-up.

\section{Participants}

Six consecutive patients who were referred for outpatient behavioral rehabilitation, and who reported substantial fear of movement/(re)injury (Tampa Scale for Kinesiophobia [TSK: Kori et al., 1990] score >39), were included in the study. The cut-off was based on the median of the TSK distribution of chronic low back pain samples reported earlier. Other inclusion criteria were non-specific low back pain for at least 6 months and age between 18 and 65. Exclusion criteria were illiteracy, pregnancy, alcohol or drug abuse, and serious psychopathology based on the Symptom Checklist of which Dutch norms are available. Due to a major depressive disorder, a posttraumatic stress disorder, pregnancy, and insufficient fluency in the Dutch language, 4 of 10 consecutive patients with pain-related fear were excluded.

\section{Procedure and program overview}

Patients who met the selection criteria received a physical examination and were subsequently invited for a semi-structured interview with the psychologist, during which a cognitive and behavioral analysis of the pain problem was made.

The Photograph series of Daily activities (PHODA: Kugler et al., 1999) was used to develop a graded hierarchy of feared activities. The patient was requested to place each of the 98 photographs along a vertical line with 11 anchor points (ranging from 0 to 100 ) printed on a $100 \mathrm{~cm} \times 40 \mathrm{~cm}$ hardboard. This fear thermometer was placed on a table in front of the patient with the following instruction: "Please watch each photograph carefully, and try to imagine you performing the same movement. Place the photograph on the thermometer according to the extent in which you feel that this movement is harmful to your back."

After this assessment, all patients started with the baseline period $(A)$, during which they completed daily measurements at home. This period was followed by EDU, followed again by a 3-week nontreatment period (B). Then, random assignment either to 24 hours of GEXP (C) spread over 6 weeks or to 32 hours of OPE (D) spread over 8 weeks occurred.

\section{Education (EDU)}

During the first part of the EDU, diagnostics tests were being reviewed together with a physician. The physician explained to the patients that their pain must be viewed as a common condition that can be self-managed, rather than as a serious disease or a condition that needs careful protection. In cases where the pain-related fear was also fuelled by the visual confrontation with diagnostics tests, patients 
were explained that they probably have overestimated the value of these tests, and that in symptom-free people, similar abnormalities can be found (Jensen et al., 1994). One of the major goals of the second part of the EDU was to increase the willingness of the patient to finally engage in activities they have been avoiding for a long time. The aim was to correct the misinterpretations and misconceptions that have occurred during the development of the pain-related fear. The patient was given careful explanation of the fear-avoidance model. By using the patient's individual symptoms, beliefs, and behaviors, it was illustrated how vicious circles (paincatastrophic thought-fear-avoidance-disability-pain) maintain the pain problem.

\section{Graded exposure in vivo (GEXP)}

Based on the graded hierarchy of fear-eliciting situations, individually tailored practice tasks were developed. There was also one exposure session that actually took place in the work or home situation. To challenge the validity of catastrophic assumptions and misinterpretations, behavioural experiments were used during GEXP. A detailed description of GEXP can be found in Vlaeyen et al. (2002c).

\section{Operant graded activity program (OPE)}

This program was based on the operant principles described by Fordyce (1976) and Lindström et al. (1992). It was aimed at increasing healthy behaviors and decreasing pain behaviors. To avoid contamination between the GEXP and the OPE, activities that were placed above 50 on the thermometer of the PHODA were excluded from the OPE. The team members monitored this without notifying the patient about this rule. The rationale provided to the patients was that inactivity may lead to disuse, which often promotes pain, and that increasing of the activity level and muscle strength consequently is likely to prevent future disability.

\section{Outcome measures}

\section{Daily measures}

To check whether the GEXP indeed modified the fear appraisals, pain intensity, and activity goal achievement, a short questionnaire was used consisting of 11 visual analog scales (VAS; from 0 to 10) with items representing main factors of existing questionnaires for pain-related fear and catastrophizing. These items were meant as manipulation check. Three main scores of part 1 were derived, consisting of the mean scores (range 0-10) of the items from the TSK, the Pain Anxiety Symptoms Scale (PASS: McCracken et al., 1992), and the Pain Catastrophizing Scale (PCS: Sullivan et al., 1995). Daily pain intensity was measured with an additional VAS anchored with "no pain at all" at one extreme and "worst pain experienced" at the other. The last 3 VAS referred to the performance of personally relevant activities. Each scale consisted of the same question: "How difficult was it to perform this activity to- 
day?" The scale was anchored with "no problem at all" at one extreme and "impossible" at the other. This measure was administered on a daily basis during the whole duration of the study and the follow-up period of 1 week. The patients were instructed to complete the scales each evening and to send the package the next day to the researchers. This measure has been shown to be sensitive to GEXP in previous studies (Vlaeyen et al., 2001; 2002a; 2002b).

\section{Questionnaires}

The Dutch version of the TSK was used. Vigilance for pain sensations was measured with the Dutch version of the Pain Vigilance and Awareness Questionnaire (PVAQ: McCracken, 1997; Roelofs et al., 2002). Patients also completed the Dutch version of the Roland Disability Questionnaire (RDQ: Deyo et al., 1998; Roland \& Morris, 1983).

\section{Activity monitor}

Patients were requested to carry an ambulatory activity monitor (uniaxial accelerometer) attached to a belt dorsally, close to lumbar discs L4 and L5 (Bussman et al., 1998). Movement counts were registered for an entire week during the moment of get up and bedtime, except for activities involving contact with water. The patients registered carrying times and the kind of activities performed by the help of a diary. At termination of the registration period, the patient returned the device, and the data were downloaded to a personal computer. Movement counts were added and subsequently divided by the time the accelerometer was carried. The activity monitor was carried 4 times for an entire week.

\section{Statistical analyses}

For analyzing the resulting data of the daily measures, a randomization test for single case experimental designs using the rationale of Edgington (1995) was carried out. Because intervention $B$ was expected to be superior to baseline $A$, and intervention $C$ was expected to be superior to $A$ and intervention $B$, the null hypothesis that there is no differential effect for any of the measurement times was tested using a randomization test on the differences between $B$ and $A, C$, and $A, C$ and $B, D$, and $A, D$ and $B$. Although the follow-up $(F)$ was expected to be superior to $A$ and would not change in relation to $C$ and $D$, differences between $F$ and $A, F$ and $C, F$ and $D$, were also tested using randomization tests. The analyses were performed using the SCRT software (Single-Case Randomization Tests, version 1.1: Onghena \& Van Damme, 1994). The program also allowed the calculation of a combined $P$ value of each design when the cases were considered simultaneously in a meta-analysis according to Edgington's additive method. 


\section{Preset criteria for non-daily measures}

To conclude whether the treatment could be considered successful, the authors decided to formulate preset criteria partly based on existing norms. For the TSK and the PVAQ, a reduction of more than 30 percentile points was considered relevant.

\section{Treatment group ABC}

Fear of movement / (re) injury

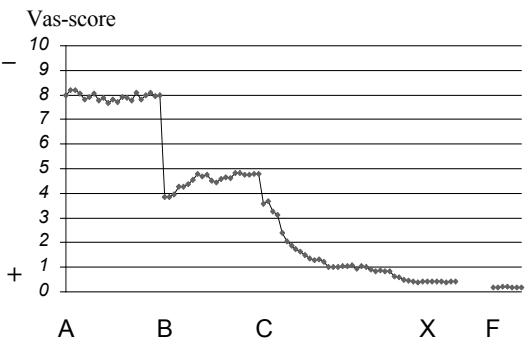

Pain catastrophizing

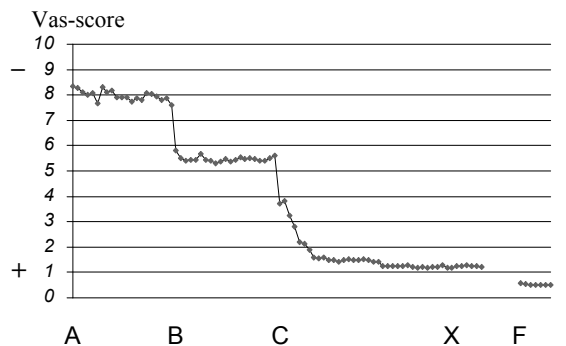

Fear of pain



$A=$ Start BAS (21 days no-treatment period)

$B=E D U$ (followed by a 21 days no-treatment period)

$C=$ Start GEXP $(12,5$ hours over 35 days $)$

$X=$ End GEXP (followed by 7 days activity monitor)

$\mathrm{F}=\mathrm{FU}$ (6-month follow-up period of 7 days)
Treatment group ABD

Fear of movement / (re) injury
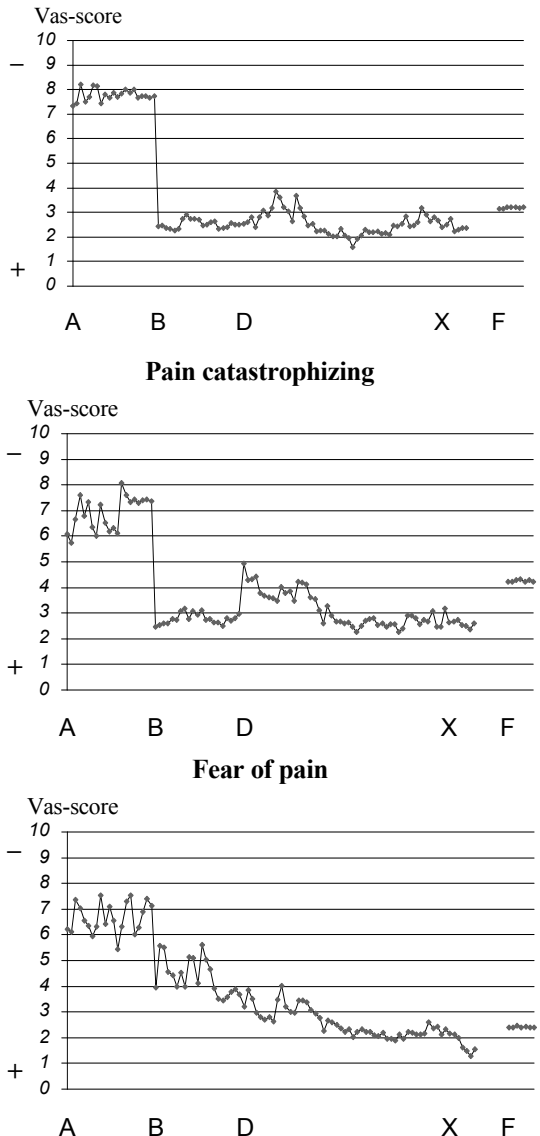

$\mathrm{D}=$ Start OPE (35 hours over 49 days)

Figure 1. Mean daily measures of fear of movement/(re)injury, pain catastrophizing and fear of pain, across baseline (BAS: 21 days), psycho-education (EDU: 21 days), graded exposure in vivo (GEXP: 12,5 hours over 35 days) in treatment group $A B C$, operant graded activity program (OPE: 35 hours over 49 days) in treatment group $A B D$, and 6-month follow-up period (FU: 7 days). 
Treatment group ABC

patient 1

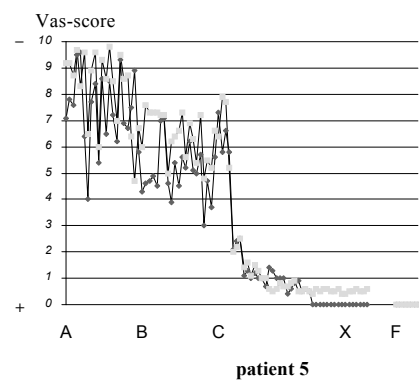

$\rightarrow$ cycle

Vas-score

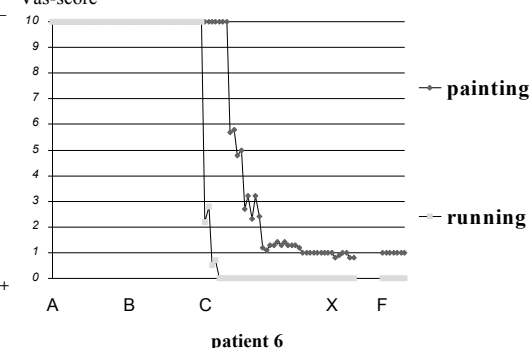

patient 6

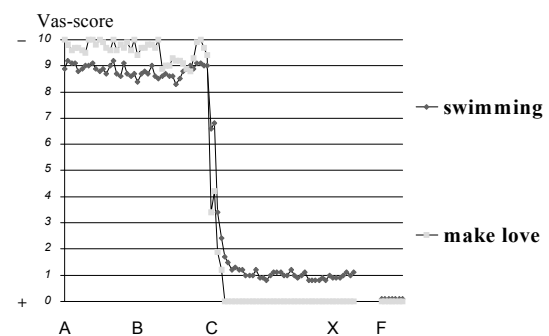

$A=$ Start BAS (21 days no-treatment period)

$\mathrm{B}=\mathrm{EDU}$ (followed by a 21 days no-treatment period)

$C=$ Start GEXP $(12,5$ hours over 35 days $)$

$X=$ End GEXP (followed by 7 days activity monitor)

$\mathrm{F}=\mathrm{FU}$ (6-month follow-up period of 7 days)
Treatment group ABD

patient 2

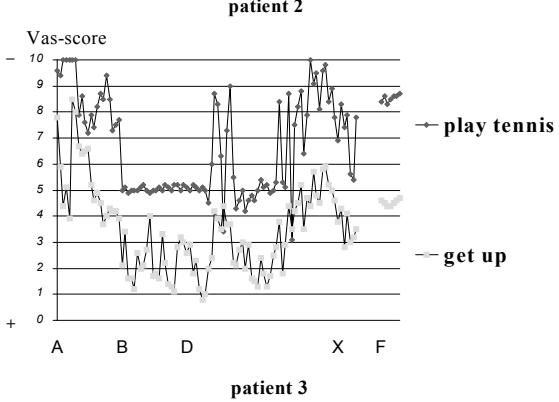

Vas-score
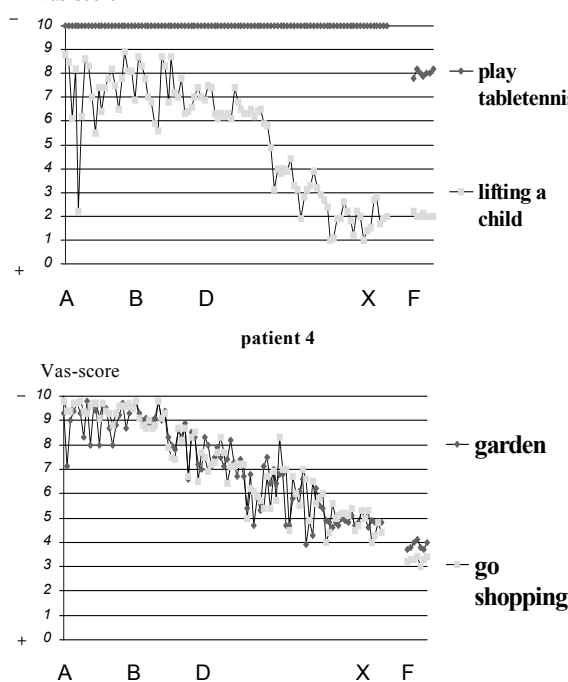

A $\quad B \quad X \quad F$

$D=$ Start OPE (35 hours over 49 days)

Figure 2. Mean daily measures of self-reported difficulties in performance of activities at home, across baseline (BAS: 21 days), psycho-education (EDU: 21 days), graded exposure in vivo (GEXP: 12,5 hours over 35 days) in treatment group $A B C$, operant graded activity program (OPE: 35 hours over 49 days) in treatment group $A B D$, and 6-month follow-up period (FU: 7 days).

In line with Stratford et al. (1998), we decided that a change score of 5 can be clinically relevant for the RDQ. For the PHODA, no norms were available. Therefore, we estimated that a $50 \%$ decrease would give enough support that the treat value of the activities used in both treatment programs had decreased. For the ambulatory activity monitor, standardized z-scores were calculated for each patient individually by subtracting the mean number of baseline counts and dividing these values by the 
baseline standard deviation for that individual. This was done for mean counts in the week after EDU and the treatment module. We estimated that an increase of 5 z-scores could be clinically relevant.

\section{Results}

\section{Daily measures}

Figure 1 shows the patterns of change of the mean daily VAS ratings for fear of movement/(re)injury, pain catastrophizing, and fear of pain for the different treatment groups. Visual inspection of the data suggest that in both treatment groups, the EDU produced substantial short-term decreases in fear of movement/(re)injury, pain catastrophizing, and fear of pain. Visual inspection also reveals that a further decrease of these dependent variables occurred when GEXP followed the notreatment period after EDU. On the other hand, when OPE followed B, there was no further decrease. The results of the randomization test procedure are displayed in table 1 and appear to confirm the conclusions of EDU. Compared with baseline A, significant changes are found in fear of movement/(re)injury, pain catastrophizing, and fear of pain when EDU takes place. In addition to this, significant changes are also found in pain catastrophizing and fear of pain when GEXP (C) is compared with $B$ and not when OPE (D) is compared with $B$. Notable is that particularly in treatment group $A B C$, all improvements remained during the follow-up period.

Of interest, and quite unexpectedly, is that in treatment group $A B C$, a significant reduction in pain intensity occurred during follow-up as compared with baseline (period A) (table 1).

Visual inspection of the self-reported difficulties in performance of daily activities at home (figure 2) shows that they remained unchanged during EDU (period B) and only decreased in the patients who received GEXP (period C). According to the graphical display, the randomization test on the raw data showed that the change in performance occurred in treatment group $A B C$ for all the patients during GEXP (period $C$ ), and that the improvements remained during follow-up. Compared with OPE (period $D$ ) in treatment group $A B D$, a significant change in performance occurred only in patient 2 . However, contrary to patient 2, patient 3 and 4 showed a significant change in performance during follow-up as compared with baseline (period A). Finally, in each treatment group, there was one patient were a significant change occurred after EDU (period B). 
Table $1 P$-values for the $A B C$ and $A B D$ treatment group randomization $t$-tests on the daily measures for fear of movement/(re)injury, pain catastrophizing, fear of pain and current pain intensity

\begin{tabular}{lllll}
\hline Variables & $\begin{array}{l}\text { Treatment } \\
\text { sequence }\end{array}$ & Group ABC & $\begin{array}{l}\text { Treatment } \\
\text { sequence }\end{array}$ & Group ABD \\
\hline Fear of movement/ & BAS-EDU & $0.024^{*}$ & BAS-EDU & $0.024^{*}$ \\
(re)injury & BAS-GEXP & $0.016^{*}$ & BAS-OPE & $0.013^{*}$ \\
& EDU-GEXP & 0.097 & EDU-OPE & 0.776 \\
& BAS-FU & $0.037^{*}$ & BAS-FU & $0.037^{*}$ \\
& GEXP-FU & 0.813 & OPE-FU & 1.000 \\
Pain catastrophizing & & & \\
& BAS-EDU & $0.024^{*}$ & BAS-EDU & $0.024^{*}$ \\
& BAS-GEXP & $0.016^{*}$ & BAS-OPE & $0.013^{*}$ \\
& EDU-GEXP & $0.016^{*}$ & EDU-OPE & 0.882 \\
Fear of Pain & BAS-FU & $0.037^{*}$ & BAS-FU & $0.037^{*}$ \\
& GEXP-FU & 0.292 & OPE-FU & 1.000 \\
& & & & \\
& BAS-EDU & $0.024^{*}$ & BAS-EDU & $0.024^{*}$ \\
Current pain & BAS-GEXP & $0.016^{*}$ & BAS-OPE & $0.013^{*}$ \\
& EDU-GEXP & $0.016^{*}$ & EDU-OPE & 0.184 \\
& BAS-FU & $0.037^{*}$ & BAS-FU & $0.037^{*}$ \\
& GEXP-FU & 0.500 & OPE-FU & 0.968 \\
& BAS-EDU & 0.184 & BAS-EDU & 0.390 \\
& BAS-GEXP & 0.677 & BAS-OPE & 0.197 \\
& EDU-GEXP & 0.667 & EDU-OPE & 0.803 \\
& BAS-FU & $0.037^{*}$ & BAS-FU & 0.074 \\
& GEXP-FU & 0.271 & OPE-FU & 0.161 \\
\hline
\end{tabular}

${ }^{*} p<0.05 ;{ }^{+}$The intervention point was determined randomly with a minimum phase length of 1 ; Bas, baseline; EDU, education; GEXP, graded exposure in vivo; OPE, operant graded activity program; FU, 6month-follow-up

\section{Questionnaires}

\section{Pain-related fear}

Table 2 shows that the mean TSK scores in both treatment groups decreased after EDU (period B) and even decreased to a mean score of 21 (<10th percentile) when the GEXP (period C) was delivered and not the OPE (period D). For PHODA, a drastic reduction was observed at the end of GEXP (period $C$ ) as compared with baseline (period A) and EDU (period B) and remained at follow-up. 
Table 2 Mean scores of fear of movement/(re)injury (TSK), pain vigilance (PVAQ), self-reported disability (RDQ), and fearfulness of movements (PHODA), taken at baseline, before and after each first treatment module, and at the 6 month follow-up for treatment groups $A B C(N=3)$ and $A B D(N=3)$

\begin{tabular}{lllll}
\hline Questionnaires & Treatment & Group ABC & Treatment & Group ABD \\
\hline Tampa Scale for Kinesiophobia & Baseline & 41 & Baseline & 46 \\
[TSK] & Education & 29,33 & Education & 30 \\
& Start GEXP & 33,33 & Start OPE & 31,3 \\
& End GEXP & 21,33 & End OPE & 31,3 \\
& Follow-up & 20,33 & Follow-up & 33 \\
& & & & \\
Pain Vigilance and Awareness & Baseline & 49,33 & Baseline & 50,3 \\
Questionnaire [PVAQ] & Education & 44,67 & Education & 45 \\
& Start GEXP & 41 & Start OPE & 44,67 \\
& End GEXP & 18,33 & End OPE & 35,67 \\
& Follow-up & 19,67 & Follow-up & 37,33 \\
Roland Disability Questionnaire & Baseline & 15 & & \\
[RDQ] & Education & 12,67 & Baseline & 16,33 \\
& Start GEXP & 14,67 & Education & 11,67 \\
& End GEXP & 2,67 & End OPE & 15,67 \\
& Follow-up & 2 & Follow-up & 15,67 \\
& & & & \\
The Photograph series of Daily & Baseline & 68,33 & Baseline & 71,33 \\
activities [PHODA] & Education & 66 & Education & 72,33 \\
& Start GEXP & 68,67 & Start OPE & 72 \\
& End GEXP & 11,67 & End OPE & 59 \\
& Follow-up & 12 & Follow-up & 58,67 \\
\hline
\end{tabular}

\section{Pain vigilance}

As compared with baseline (period A), table 2 shows that the decrease of the mean score of the PVAQ at the end of EDU (period B) was not relevant in both treatment groups. However, under the influence of both GEXP (period C) and OPE (period D), the decrease of the mean score in both treatment groups was clinical relevant. Hereby, it is notable that in treatment group $A B D$, the reduction of $30 \%$ was, compared to baseline (period $A$ ) and EDU (period $B$ ), just managed by OPE (period D) and that in group $A B C$, a reduction of $70 \%$ was observed after GEXP (period $C$ ).

\section{Pain disability}

For the RDQ, table 2 shows that relevant changes are observed when both GEXP (period C) and OPE (period D) were introduced. Overall, RDQ scores decreased from a mean of 15 to a mean of 2 in group $A B C$ and from 16 to 6 in group ABD. These differences largely exceed the preset criterion of 5 . 


\section{Activity monitor}

In accordance with the self-reported difficulties in performance of personal relevant daily activities at home, figure 3 shows that the mean standardized scores of the activity monitor data did not change after EDU (period B) and only increased clinically relevant in group ABC during GEXP (period C).

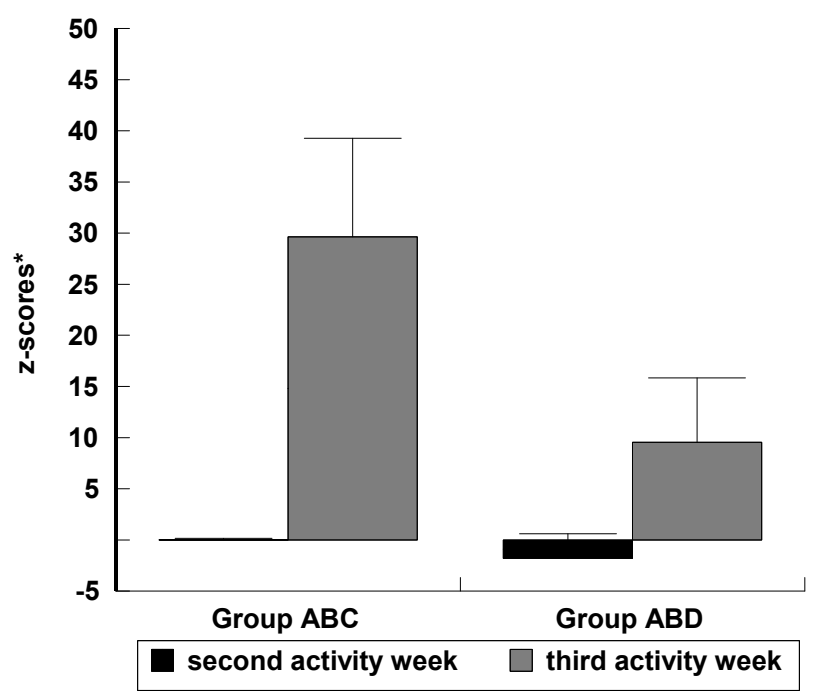

Figure 3. Activity monitor data. Mean standardized z-scores (and standard error bars) for patients who received intervention $A B C$ (baseline-education-exposure) and intervention $A B D$ (baseline-educationoperant graded activity program); ${ }^{*}$ z-score is based on intra-person baseline mean and SD.

\section{Discussion}

The aim of this study was to examine the effects of a single educational session followed by actual exposure in vivo or operant graded activity. Six consecutive patients with low back pain who were referred for outpatient behavioral rehabilitation and reported substantial fear of movement/(re)injury were included. A single-case experimental design was applied in which chronically disabled patients were randomized over two cognitive-behavioral treatments. Both the randomization tests on the daily measures and pre-post assessments showed that after education and the following no-treatment period, subjective ratings of pain-related fear and catastrophizing decreased substantially in all patients. This suggests that a highly individualized and tailored response to a personal formulation of a patient's problem within a substantive model has good empirical support in changing patient's perceptions about the harmfulness of physical activity and threat value of pain and is supposed to be part of CBT. Another point to make here is that our educational session 
does not appear to be a simple reassurance approach. On the whole, reassurance seems to have a transient impact with an immediate reduction of worry and concern followed by a rebound to pre-intervention levels within 24 hours or so. In reducing pain-related fear, the results of the current study suggest that our education works in 1 session. This implicates that it would be a really effective intervention to use in primary care if one could identify at-risk patients.

However, despite the effect of education on pain-related fear, the self-reported difficulties in performance of daily activities at home and the subjective ratings of the PHODA remained unchanged during that period. These variables only decreased in the patients who received the graded exposure in vivo. This suggests that education is not powerful enough to change actual escape and avoidance behavior. Why education only influences beliefs about pain and is not actual escape and avoidance behavior is an intriguing question, which merits further examination. There is growing evidence that attitudes toward physical activity, and the role of implicit versus explicit affective evaluations, play an important role in the treatment outcomes of chronic low back pain. Studies have shown that especially highly accessible attitudes such as the deeply ingrained fear of movement/(re)injury will evoke automatic activation from memory when the object is encountered (Fazio, 1986; Sanbonmatsu \& Fazio, 1990; Sarafino, 1998). If patients have labeled attitude objects as positive, approach behavior will occur. Conversely, avoidance behavior is elicited when attitude objects have acquired a negative valence. Furthermore, it is possible that individuals can hold "dual attitudes" which result from different evaluations of the same attitude object, one of which is an automatic, implicit attitude and the other being an explicit one (Ajzen, 2001; Bohner \& Wänke, 2002; De Jong et al., 2003; Hermans et al., 2002a; Wilson et al., 2000). The following citation from one patient in the current study is a nice illustration: "After the educational session, I realized that my concerns about (re)injury were irrational, and that I had avoided a lot of activities the last few years for no reason. However, when my wife asked me to go out for a ride on the bike yesterday, I immediately said no." Consequently, it might be expected that the dimensionality and thus the complexity in attitudes have an impact on (speed of) the change of avoidance and escape behavior.

Another possible reason for the lack of association between beliefs and behavior is the use of self-report questionnaires. It has been suggested that self-reports, as explicit measures, assess what are called "self-attributed motives" and that they may be clouded with demand characteristics, self-presentational bias, or attributional a priori theories (De Jong, 2002; Fazio, 1986; Fazio et al., 1995; Nisbett \& Wilson, 1977; Sherman et al., 2004). As a consequence, it could be that self-attributed motives are akin to explicit attitudes that are expressed when people are asked directly 
how they feel, and that implicit motives, assessed by the activity monitor, are similar to habitual implicit attitudes, in that they automatically influence behavior.

The finding that the activity monitor data follow the same picture as the daily selfreport measures on activity achievement supports the assumption that actual exposure to fear-eliciting activities and/or movements is necessary to modify escape and avoidance behavior due to pain-related fear. It also suggests that the confrontation of fear-eliciting activities in the treatment setting is analogue for how patients respond in daily life situations. Moreover, treatment gains produced during EXP seem to generalize to the home setting and in the absence of therapists.

Although the results show that exposure in vivo with behavioral tests are superior to an operant-behavioral graded activity treatment in this subgroup of patients expressing substantial fear of movement/(re)injury, there are a number of caveats to be considered. First, this study is limited in that it included only 6 patients. On the other hand, a single-case experimental design was chosen with appropriate randomization tests. Second, during the exposure in vivo, behavioral tests have been developed. Theoretically, it is possible that the effects of the exposure in vivo treatment are due to this component. However, in practice, behavioral tests are difficult to separate from mere exposure, and they can best be used simultaneously. In any case, the findings of this study contribute to the idea that interventions for patients with chronic low back pain with substantial pain-related fear have to be tailored to the specific attitudes of the patient in question. 



\section{Chapter 6}

\section{Generalization of Graded Exposure in Vivo in Complex Regional Pain Syndrome type I (CRPS-I)}

Jeroen R de Jong, Marlies den Hollander, Isis Bulté, Joop Ruijgrok, Johan WS Vlaeyen. Generalization of Graded Exposure in Vivo in Complex Regional Pain Syndrome type I (CRPS-I). Submitted for publication 


\begin{abstract}
Using a randomized replicated single case experimental design, this study examined whether extinction of pain-related fear during graded exposure in vivo (GEXP) in fearful patients with Complex Regional Pain Syndrome type I (CRPS-I) of the upper extremities, generalized better to a new movement when subjects were exposed to multiple stimuli during the treatment as compared to an exposure treatment in which patients were repeatedly exposed to a smaller number of activities. In the Multiple Stimuli condition (MSTI, N = 4), GEXP consisted of at least 15 activities to which patients were exposed once, and in the Multiple Exposure condition (MEXP, $\mathrm{N}=4$ ), patients were exposed to 3 activities to which patients were exposed five times each. In both conditions generalization of GEXP was tested by exposing patients to a new fearful activity. It was hypothesized that generalization of extinction is facilitated in MSTI. In contrast to the expectations, patients of both conditions performed equally well in the test exposure, showing generalization to a new stimulus both immediately after GEXP and during 6-month follow-up. Overall, it seems that, irrespective of the kind of exposure, extinction generalized to new threatening activities. Possible mechanisms of GEXP in relation to generalization are discussed.
\end{abstract}




\section{Introduction}

Pain-related fear has been identified as one of the most potent predictors of longterm pain-related disability (Leeuw et al., 2007a; Vlaeyen \& Linton, 2000). Pain is an evolutionary hard-wired experience that interrupts ongoing activities and captures attention to protect the individual from further injury. In the short term, defensive behaviors such as escape and avoidance from painful activities after injury are adaptive and allow the injury to heal and pain to subside. However, prolongation of these behaviors may have the paradoxical effect that fear and associated disability sustains. The unnecessary fear of engaging in rehabilitative efforts, leading to preoccupation with bodily symptoms, is often associated with catastrophic (mis)interpretation of persistent pain, in terms of most extreme negative consequences, such as the belief that activity might aggravate the initial injury and thus must be harmful (Asmundson et al., 1999; Turk \& Okifuji, 2002, Leeuw et al., 2007a; Sullivan et al., 2005). Pain catastrophizing is associated with increased reporting of pain, higher levels of disability, and higher levels of health care use, longer hospitalizations, increased pain medication usage, and higher levels of motor pain behaviors (Keefe et al., 2004). Furthermore, catastrophic thinking inevitably results in painrelated fear, which may cluster around interoceptive (e.g. pain itself), proprioceptive (e.g. specific activities/movements) or exteroceptive (e.g. work environment) stimuli. Pain-related fear often causes a cascade of psychological and physical events which in turn can perpetuate pain problems (Leeuw et al., 2007a; Vlaeyen \& Linton, 2000).

Exposure therapy is the preferred treatment of various kinds of anxiety disorders (Cox et al., 1992). Recent clinical experimental studies and clinical trials have shown that graded exposure in vivo is also a successful treatment for chronic pain patients presenting a pain-related fear (Boersma et al., 2004; De Jong et al., 2005a, 2005b; Leeuw et al., 2008; Linton et al., 2007; Vlaeyen et al., 2001, 2002a, 2002b; Woods \& Asmundson, 2008). During exposure, a former conditioned stimulus (CS: specific activity) is presented several times during which the representation of the unconditioned stimulus (UCS: pain) changes. The individual learns that a specific activity no longer predicts the catastrophic consequences, for example, pain is no longer a sign of injury. During exposure the meaning of pain changes to a less threatening UCS. Despite the success of exposure in chronic pain it is still the question if extinction generalizes to new threatening activities. Return of fear is a problem frequently encountered after successful exposure treatment (Hermans et al., 2005). The evidence so far (Baeyens et al., 2005; Bouton \& Swartzentruber, 1991; Craske et al., 2008; Hermans et al., 2005; Rachman, 1989; Vansteenwegen et al., 2007) suggests that extinction cannot be equated with 'unlearning' but rather the learning of exceptions to the rule that physical activity is dangerous. The fear response can spon- 
taneously arise after a while (spontaneous recovery), when confronted with a new CS-US event (rapid reacquisition), or with an unpredicted UCS (reinstatement). A study by Rescorla (1996) shows that exposure does not alter the acquired CS-US association, suggesting that extinction is context dependent (Bouton \& King, 1983; Craske et al., 2008; Hermans et al., 2005; Vansteenwegen et al., 2007).

A number of studies have shed light on the generalization of extinction in chronic pain patients. First, Goubert et al. (2002) investigated whether the effects of exposure to one movement generalize towards another dissimilar movement. Patients were requested to perform two movements twice. Analyses revealed that patients initially over-predicted pain, but after exposure the over-prediction was readily corrected. However, this exposure effect did not generalize from the first to the second movement. Additionally, the lack of generalization was only characteristic for patients reporting a high level of catastrophic thinking about pain. In a subsequent study where ratings for perceived harm were also obtained, similar results were reported (Crombez et al. 2002). Secondly, Goubert et al. (2005) investigated the benefits of exposure to a variety of movements versus exposure to the same movements on the correction of over-prediction of pain and behavioral performance on a final behavioral test which was the same for all the patients. The results showed that patients over-predicted pain during a threatening behavioral test. No support was found for the hypothesis that varied exposure facilitates generalization of exposure effects. Contrary to these studies, Trost et al. (2008) showed successful generalization of pain expectancy corrections across four adaptations of a reaching task, each introducing an element of increased intensity, in chronic low back pain patients reporting high levels of pain-related fear.

The results of the above-described studies in back pain patients showed that extinction of pain-related fear is a laborious process that poorly generalizes to other movements or contexts. Extinction is not 'unlearning', but learning something new. Patients learn conceptual exceptions to their general rule: 'This movement is harmful'. From this point of view it seems important to expose fearful individuals to stimuli that were encountered during the acquisition of fear, and to provide adequate exceptions so that these exceptions will form the general rule. However, in rehabilitation medicine the question is how many exceptions should be offered during exposure therapy to be able to function in normal life? If generalization of extinction is so hard to accomplish, will pain patients reporting high levels of painrelated fear only learn that they do not have to be fearful for the activities that are performed during the exposure sessions?

The primary objective of this study was to replicate the finding that exposure to multiple movements promotes generalization of extinction to new stimuli. Using a 
replicated single case experimental design, we decided to experimentally examine whether extinction of pain-related fear during a graded exposure in vivo program (GEXP) in patients with Complex Regional Pain Syndrome type I (CRPS-I) reporting high levels of pain-related fear generalized to threatening activities to which they were not exposed during GEXP. In a previous study of De Jong et al. (2005a), results showed that GEXP seems to be a successful treatment in CRPS-I patients. Two GEXP conditions of 15 sessions were tested: a Multiple Stimuli condition (MSTI) in which the exposure therapy consisted of 15 activities to which the patients were exposed once, and a Multiple Exposure condition (MEXP) of 3 activities to which the patients were exposed five times. Our expectation was that as compared to patients in MEXP, the patients in the MSTI condition experienced more 'exceptions to the rule', as a result of which they would show less fear when exposed to a new movement.

\section{Materials and methods}

\section{Study design}

A randomized replicated sequential single-case experimental $A B C$-design was used (Onghena \& Edgington, 2005). Patients were randomly assigned to one of the two exposure conditions (period $B$ ). Randomisation occurred after the baseline (BAS; period $A$ ) and was done by a computer system providing allocations in a locked, unreadable file that could be assessed only by an independent research administrator. In MSTI, GEXP consisted of 15 activities to which the patients were exposed once and in MEXP of 3 activities to which the patients were exposed five times. Also the length of the baseline was randomized and varied between 12-16 days. Finally, at 6-months there was a follow-up period of two weeks (FU; period C).

\section{Participants}

Eight consecutive patients with CRPS-I to the upper extremities were included in the study. CRPS-I is a painful disabling disorder that is poorly understood. Symptoms of CRPS-I include continuing pain, allodynia, or hyperalgesia with which the pain is disproportionate to any inciting event, abnormal swelling, hair or nail growth, skin colour or temperature changes, sweating, and limited range of motion and movement disorders. The diagnosis of CRPS-I was made according to the criteria formulated by the International Association for the Study of Pain (IASP: Merskey \& Bogduk, 1994; Stanton-Hicks et al., 1995), and based on physician evaluation of objective symptoms. The patients were referred for outpatient behavioral rehabilitation at the department of rehabilitation of the University Hospital Maastricht, and they reported pain for at least 6 months and substantial fear of movement/(re)injury 
(Tampa Scale for Kinesiophobia [TSK: Miller, Kori, \& Todd, 1991] score > 39). The cut-off was based on the median of the TSK distribution of chronic low back pain samples reported earlier (Crombez, Vlaeyen, Heuts \& Lysens, 1999; Vlaeyen \& Linton, 2000). Given the findings of Goubert et al. (2002), which indicated that lack of generalization was only characteristic for patients reporting a high level of catastrophic thinking, it was hypothesized that differences in pain catastrophizing should be avoided. Therefore, patients were included if they also were characterized by high levels of pain catastrophizing (The Pain Catastrophizing Scale [PCS: Sullivan \& Bishop, 1995] score > 21). This cut-off was based on the median of the PCS distribution of chronic low back pain samples (Van Damme et al., 2000). The ages of the patients were between 18 and 65 years.

Further, exclusion criteria were illiteracy, pregnancy, impairment of contra lateral extremity (e.g. because of rheumatoid arthritis, prior sympathectomy of the affected extremity), alcohol or drug abuse, and serious psychopathology based on the Symptom Checklist (SCL-90: Arrindell \& Ettema, 1986) of which Dutch norms were available. With regard to psychopathology none of the potential participants were excluded. During the study ten patients dropped out due to various reasons. Of these, four patients were excluded after the psychological intake of the GEXP because they still specified pain-reduction as their only treatment goal. GEXP was not focused on pain reduction, but to improve overall functioning despite the pain. Two patients declined participation to the study, because they refused to terminate ongoing treatment. Two patients dropped out during the exposure in vivo sessions, of which one had to keep rest after heart problems were diagnosed and the other because of family conflicts. Finally, two patients were excluded the end of GEXP. Both patients had concealed the fact of an ongoing lawsuit and due to this situation not completed the questionnaires appropriately. They were afraid that their research data would be passed on the court. Patients who dropped out from the study were replaced by new consecutive patients. Finally, eight patients were included in the study. The patients randomized to MSTI $(N=4)$ were all female with a mean age of $49 \pm 4.8$ (SD) of which three patients suffer from CRPS-I in the dominant hand and one in the non-dominant hand. In two patients there was a clear limitation of active movements, visible by a deviating position at rest of the upper extremity (claw-shaped fingers or hyper extension of the MCP's). One patient randomized to $\operatorname{MEXP}(\mathrm{N}=4)$ was male and the other three were female. Their mean age was $39 \pm$ 11.0 (SD). Also in MEXP, three patients suffered from CRPS-I in the dominant hand and one in the non-dominant hand. In one patient there was a clear limitation of active movements. At inclusion, the mean duration of the complaints was 3.5 years (range: 1.5 years -8 years). 


\section{Procedure and program overview}

The Medical Ethics Committee of the University Hospital Maastricht approved the research protocol. Patients were first evaluated by the rehabilitation physician who conducted a full physical examination, evaluated previous diagnostic tests, and informed participants about the study. When patients agreed to participate, the researcher sent additional written information, along with an informed consent form, TSK, PCS and SCL-90. If patients fulfilled the pre-set inclusion criteria, they started with the baseline measurement period (BAS).

To test whether the effects of GEXP generalized to other not presented threatening activities, patients performed a behavioral test under supervision of an independent occupational therapist. This was done just after GEXP as well as during FU. In addition, at the start of BAS and after the above-mentioned behavioral tests, the same independent occupational therapist observed and evaluated, to what extent the affected arm/hand was involved in a series of standardized daily activities using the Radboud Skills Test (RST: Cup, van de Ven-Stevens \& Corstens-Mignot, 1999). These activities were not performed during GEXP. Besides the RST also standardized questionnaires were completed. Further, during BAS, GEXP and two weeks after GEXP and FU patients completed daily measures at home.

\section{Graded exposure in vivo (GEXP)}

The same outpatient therapist team provided GEXP in both conditions. The team consisted of a behavioral therapist and an occupational therapist both experienced in the cognitive-behavioral rehabilitation of patients with chronic pain. In both conditions, GEXP was highly structured, protocolized, individually tailored, and aimed to restore a normal pattern of daily function, including complete return to work. Pain reduction and decrease of the observed and reported physiological signs was not a direct goal.

The GEXP started with a psychological intake by the behavioral therapist aimed to complete a behavioral analysis of the pain problem with special attention to patient's catastrophic interpretations of the pain problem. After the psychological intake (one session) an educational session was provided by the therapist team explaining the treatment rationale to each patient individually. Patients were given a careful explanation of the fear-avoidance model using their own individual symptoms, beliefs and behaviors in relation to their pain complaints. The therapist team illustrated the paradoxical and dysfunctional effects of various safety behaviors, and offered the patient a new view on pain as a common condition that can be selfmanaged, rather than as a serious disease or a condition that needs careful protection. One of the major goals of the educational component was to help the patient understand that the consequences of pain are catastrophically overestimated, and that various safety behaviors are not adaptive anymore. Directly after the explana- 
tion of the treatment rationale, a hierarchy of fear-eliciting movements and activities was made using the Photograph series of Daily Activities for the upper extremities (PHODA-UE: Dubbers, Vikström \& de Jong, 2003). Patients were requested to judge the harmfulness of 125 physical movements from daily life activities represented by photographs. Using a (fear) thermometer, each picture was given a rating between zero (representing the situation which was not harmful for the upper extremity) to 100 (representing the situation which was absolutely damaging the upper extremity). Based on the graded hierarchy of the PHODA-UE individually tailored behavioral experiments were developed. For MSTI these were at least fifteen activities and for MEXP only three activities. To be sure that all activities were quite threatening in both conditions, only activities rated $>50$ on the thermometer were included. Subsequently, during the next sessions patients were gradually and systematically exposed to these tailored and fear-provoking activities. To enhance generalization and maintenance exposure was provided to the maximum spectrum of contexts and natural settings that can be achieved in and around the hospital setting. A more detailed description of GEXP can be found in Vlaeyen, De Jong, Leeuw \& Crombez (2004).

\section{Measures}

\section{Fear of movement/(re)injury}

The Dutch language version of the Tampa Scale for Kinesiophobia (TSK-DV: Goubert et al., 2000) was used to quantify fear of movement/(re)injury. The TSK-DV consists of 17 statements that have to be rated on a four-point scale ( $1=$ strongly agree, $4=$ strongly disagree). The total score was calculated after inversion of items 4, 8, 12, and 16 which are phrased in reversed key. The total score varied between 17 and 68. The Dutch version of the TSK has been shown to be sufficiently reliable and valid (Goubert et al., 2004, Roelofs et al., 2007).

\section{The perceived harmfulness of daily activities}

To measure the threat-value of daily activities the Photograph Series of Daily Activities of the upper extremities was used (PHODA: Dubbers et al., 2003) The PHODA included 125 photographs of various daily activities. Patients had to indicate to what extent they perceived these daily activities to be harmful and/or threatening. The PHODA focussed on patient's judgment about the consequences of certain movements. A thermometer consisted of a vertical line anchored at the bottom with 0 which means 'not harmful' and at the top with 100 which means 'very harmful' was used. Each photograph was given a rating according to its position on the thermometer. A mean total score ranging from 0 to 100 was calculated as the sum of each rating divided by 125 . The PHODA has been used as a measurement instru- 
ment in several studies that examined the effectiveness of graded exposure in vivo (e.g. De Jong et al., 2005a, 2005b; Leeuw et al., 2008).

\section{Pain catastrophizing}

The degree of pain catastrophizing was measured by the Pain Catastrophizing Scale (PCS: Sullivan, Bishop \& Pivik, 1995). In this study, the Dutch version of the PCS was used (PCS-DV: Van Damme, Crombez, Bijttebier, Goubert \& Van Houdenhove, 2002). The PCS instruction asked the participants to reflect on past painful experiences and to indicate the degree to which they experienced each of the thirteen thoughts or feelings when they were experiencing pain on a 5-point Likert scale $(0=$ not at all, $4=$ all the time). The PCS-DV has been shown an highly reliable and valid measure of thinking in catastrophes (Van Damme et al., 2002).

\section{Behavioral test}

In order to test generalization each patient performed a behavioral test after GEXP (post-test) and during FU, consisting of two activities. The first activity consisted of a fall forward, which should be absorbed by the hands. This activity was standardized for all patients and was done at post-treatment as well as at FU. The second activity was a random selection of one of four 'not-likely to happen in daily-life' activities (box against a punching bag, do push-ups, give a Chinese burn [it involves gripping the top of somebody's forearm with both hands then rotating the hands in opposite directions, thus stretching the skin], hang on a climbing rack). These activities were drawn from intake information of a previous study in CRPS-I patients (de Jong et al., 2005a). The activity selected at FU was always different from the one selected at post-treatment. The tests were performed under supervision of an independent occupational therapist. The perceived harmfulness of these activities was rated at baseline and just before and immediately after the performance at post-treatment and at FU.

\section{Functional disability}

Functional disability was measured with the Radboud Skills Test (RST: Cup et al., 1999) and the Radboud Skills Questionnaire (RASQ: Oerlemans et al., 2000b). The aim of the RST is to observe and evaluate to what extent CRPS-I patients involve their affected arm/hand during activities which normally are done two-handed: walk, put a letter in an envelope, put a pillow in a pillow-cover, put on a dress shirt, close a button, put on a sock, dry up the dishes, fold up a towel. The tasks were scored on a five point Likert scale ( 0 = the task is normally executed two-handed, $4=$ the task can not be executed without help). Also the way patients experienced difficulty with the task was measured. This was scored on a three point Likert scale $(0=$ no problem, 2 = a great problem). The RST provides a measure of functioning as judged by an independent occupational therapist (performing score) and the pa- 
tient himself (difficulty score). The RST has shown good psychometric properties (de Boer et al., 2001).

The RASQ, a self-report questionnaire, measures skills in which both hands were notably active. The skills, or daily activities, were described in forty-five items and divided into six domains: 1) performing abilities; 2) household activities; 3) recreational activities; 4) social activities; 5) remaining; and 6) work. A total score was computed, along with the six domain scores. Each of the items was scored on a fivepoint Likert scale ( $1=$ normal, 5 = 'I do not perform the activity anymore as a result of CRPS-I', and there was an extra score of ' 9 ' for 'not applicable'). The patient indicated the effort of the activity by choosing a number. Different scores were computed by summing up relevant items (minus the number of items in which the category 'not applicable' was chosen), divided over the number of items of the relevant scale. The questionnaire has been found reliable in CRPS-patients, with good agreement between the outcomes for test-retest and inter observer reliability studies as established with the method of Bland and Altman (1986) (Oerlemans et al., 2000b).

\section{Internal validity checks}

\section{Daily measures}

To check whether the GEXP indeed modified activity goal achievement, fear of movement/(re)injury and pain catastrophizing and also possibly pain intensity, a brief diary was used consisting of 11 items with visual analog scales. The first 7 items (table 1) represented the main factors of existing questionnaires for fear of movement/(re)injury (Tampa Scale for Kinesiophobia [TSK]: Goubert et al., 2000, 2004; Miller et al., 1991; Roelofs et al., 2004, 2007) and pain catastrophizing (Pain Catastrophizing Scale [PCS]: Sullivan, Bishop \& Pivik, 1995; Van Damme, Crombez, Bijttebier, Goubert \& Van Houdenhove, 2002). All items were scored on $10 \mathrm{~cm}$ visual analogue scales (VAS), anchored 'totally disagree' - 'totally agree'. Two main scores were derived, consisting of the mean scores (range 0 to 10) of the items from the TSK and PCS. Pain intensity was measured with an additional VAS anchored with 'no pain at all' at one extreme and 'worst pain experienced' at the other. The last three VAS referred to the performance of personally relevant activities that represented three main functional goals. Each scale was preceded by the same question: "How difficult was it to perform this activity today?" The scale was anchored with "no problem at all' at one extreme and 'impossible' at the other. The diary was completed during the whole duration of the study, and the follow-up period. The diary has been shown to be sensitive to GEXP in previous studies (e.g. De Jong et al., 2005b). 
Table 1 Items of the shortened and adapted versions of the TSK and PCS that are completed on a daily basis.

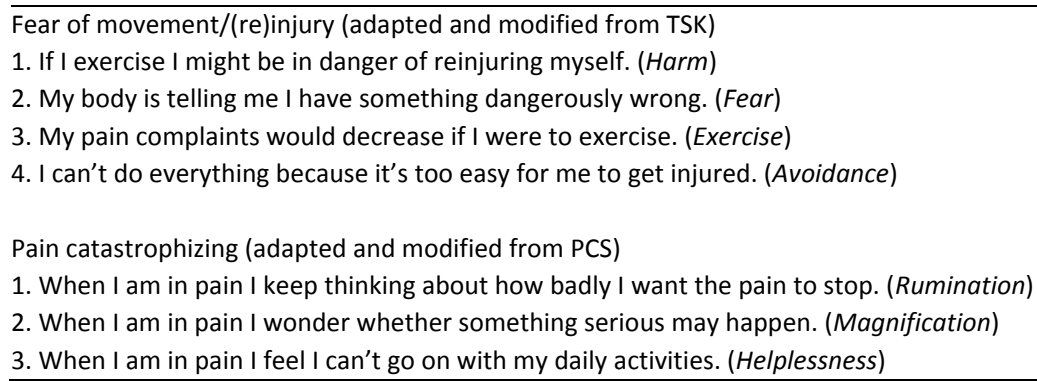

TSK, Tampa Scale for Kinesiophobia; PCS, Pain Catastrophizing Scale

\section{Treatment expectancy and credibility}

When the rationale of GEXP was explained and at the end of GEXP patients completed one expectancy and one credibility item on $10 \mathrm{~cm}$ VAS, with 'not at all' and 'very much' on the extremes: 'Do you expect that GEXP will help you to cope better with your pain complaints?' (Expectancy) and 'Do you believe that GEXP offered to you is a meaningful treatment for patients with CRPS-I?' (Credibility) (Borkovec \& Nau, 1972). This measurement was carried out by an independent occupational therapist.

\section{Statistical analyses}

For analyzing the resulting data of the diary, a randomization test based on the random determination of the moments of phase change or intervention points to test a null hypothesis about treatment effects in single case experimental designs and using the rationale of Edgington was carried out (Onghena \& Edgington, 1994). With respect to Student $t$ tests, analysis of variance $F$ tests, or other inferential procedures from within the general linear model framework, randomization tests were used because they had the advantage of being valid for single-case experiments without making distributional assumptions (Edgington \& Onghena, 2007), of being easy to apply (Edgington, 1980; Onghena \& Edgington, 2005) and of being extremely versatile for even the most complex single-case designs (Onghena \& Van Damme, 1994; Persons \& Silberschatz, 1998). The randomization tests in this study made use of the difference between means as (directional) test statistic. Replicated singlecase experiments may be considered as multiple studies that can be combined using meta-analytical procedures. Because the replicated single-case experiments in this study provided independent tests of the same null hypothesis, the directional $P$ values of these tests were combined by calculating the sum of the $P$-values and comparing this sum with all other sums that were arised under the general null hypothesis (if the null hypothesis is true, than the $P$-value is just a random draw 
from a uniform $[0,1]$ distribution). A more detailed description of the randomization tests for single-case experimental designs can be found in Onghena and Edgington (2005).

Because GEXP was expected to be superior to BAS the null hypothesis that there is no differential effect for any of the measurement times was tested using a randomization test on the differences between GEXP and BAS. While FU was expected to be superior to BAS and would not change in relation to GEXP, differences between FU and BAS and FU and GEXP were also tested using randomization tests. The analysis was performed using the SCRT software (Single-Case Randomization Tests, version 1.1: Onghena \& Van Damme, 1994).

\section{Preset criteria for non-daily measures}

For the non-daily measures (TSK, PHODA, PCS, RST and RASQ) the limited number of data made it impossible to use randomization tests. Therefore, we decided to formulate pre-set criteria to detect a clinically important change as the result of GEXP. Based on the results of previous single case studies on the efficacy of GEXP (e.g. De Jong et al., 2005a, 2005b), for the TSK and PCS, a reduction of more than 30 percentile points, and for the RASQ, a mean improvement of $50 \%$, was considered relevant. Because a number of items from the RASQ were used as activity to observe the involvement of the affected hand during the RST, by which it seemed that both instruments were strongly linked, we estimated that also a mean improvement of $50 \%$ on the RST could be supposed as clinically relevant. To detect actual changes of the PHODA it was considered that the change should exceed a variation of 20 points on the 0-100 scale (Leeuw, Goossens, van Breukelen, Boersma \& Vlaeyen, 2007b).

\section{Results}

\section{Internal validity checks}

\section{Expectancy and credibility check}

Mean expectancy ratings $(7.83 \pm 0.36$ SD and $7.10 \pm 2.14$ SD) and credibility ratings $(6.63 \pm 1.17 \mathrm{SD}$ and $7.73 \pm 2.24 \mathrm{SD})$ were relative high in both conditions, and higher as compared to a previous GEXP study in CRPS-I patients (De Jong et al. 2005). Measurements after GEXP showed a further increase of the expectancy ratings (8.23 \pm 1.47 SD and $9.43 \pm 0.48 \mathrm{SD})$ and the credibility ratings (7.98 \pm 1.66 SD and $9.15 \pm$ $0.51 \mathrm{SD})$. The results imply that the differential effectiveness of GEXP in both conditions was not substantially influenced by differences in treatment credibility and expectancy. 


\section{Daily measures}

The $P$-values of the randomization tests on the raw data of the daily measures for each patient in both conditions are displayed in table 2. Compared with BAS, after GEXP significant changes were found in fear of movement/(re)injury for each patient in MSTI and patient 5 and 6 in MEXP. Although patient 7 and 8 in MEXP reported a high score on the whole TSK during the inclusion of the study, the VASscores of the diary for the relevant variables were already low at BAS. Due to this, no significant difference between BAS and GEXP was observed. Notable is that all improvements remained during FU. The same tendency was observed for the variable pain catastrophizing with the exception of patient 8 who showed also a significant change between BAS and GEXP. With regard to personally relevant activities, the results showed a significant change in each activity after GEXP for patient 2, 3 and 4 in MSTI and patient 6 and 7 in MEXP. Also at FU these improvements remained stable. Although visual inspection of the daily measures of patient 1 in MSTI and patient 5 and 6 in MEXP suggested that GEXP had a positive impact on personally relevant activities, both at BAS, GEXP and FU the raw data were characterized by outliers, which seemed to be a possible explanation that no significant differences were found in these patients. Of interest, and quite unexpectedly, was that at FU in MSTI for each patient and in MEXP for patient 5 and 7 a significant difference could be observed for pain experience. Patient 6 showed much fluctuation in pain experience and patient 8 experienced throughout the study a lot of pain. In conclusion, based on the overall results and in accordance with its purpose, GEXP indeed modified the fear appraisals and promoted the achievement of functional goals.

\section{Outcome measures}

\section{Behavioral Test}

Table 3 and 4 provides an overview regarding the performance of the standard (headlong fall) and the pre-randomized specific activity, both considered as very threatening, during the behavioral tests observed for each patient in MSTI and MEXP. Both in behavioral test 1 (after GEXP) and 2 (at FU) all patients performed the specific activity without any safety behavior. During the behavioral test after GEXP, the independent occupational therapist observed safety behavior in one patient from MSTI during the performance of the standard activity. Conversely, during follow-up no safety behaviors were observed. On the basis of these observations it could be stated that all patients after GEXP, irrespective of the exposure condition, were able to perform threatening activities and that learning effects of GEXP generalized to threatening activities not performed during GEXP. 
Table $2 P$ Values for each patient in MSTI (GEXP: $\geq 15$ activities) and MEXP (GEXP: 3 activities) of the Randomization $t$ Test on the diary data between BAS-GEXP and BAS-FU for the dependent variables fear of movement/(re)injury (FOM), pain catastrophizing (CAT), pain experience (PE) and personally relevant activities

\begin{tabular}{|c|c|c|c|c|c|c|c|}
\hline \multirow[b]{2}{*}{ Condition } & \multirow{2}{*}{$\begin{array}{l}\text { Treatment } \\
\text { Sequence }^{a}\end{array}$} & \multicolumn{6}{|c|}{ Variables } \\
\hline & & FOM & CAT & PE & Activity 1 & Activity 2 & Activity 3 \\
\hline \multicolumn{8}{|l|}{$\overline{M S T I}$} \\
\hline \multirow[t]{2}{*}{ Patient 1} & BAS-GEXP & $0.04 *$ & $0.04 *$ & 0.08 & 0.73 (write) & 0.68 (peel) & $0.05^{*}$ (cycle) \\
\hline & BAS-FU & $0.04 *$ & $0.04 *$ & 0.07 & 0.36 & 0.09 & 0.06 \\
\hline \multirow[t]{2}{*}{ Patient 2} & BAS-GEXP & $0.04 *$ & $0.04 *$ & $0.04^{*}$ & $0.04 *$ (drive) & $0.04 *$ (cycle) & $0.04 *$ (type) \\
\hline & BAS-FU & $0.04 *$ & $0.04 *$ & $0.04^{*}$ & $0.04 *$ & $0.04 *$ & $0.04 *$ \\
\hline \multirow[t]{2}{*}{ Patient3 } & BAS-GEXP & $0.04 *$ & $0.04^{*}$ & 0.08 & $0.04^{*}$ (cycle) & $0.04^{*}(\mathrm{swim})$ & $0.04 *$ (iron) \\
\hline & BAS-FU & $0.04 *$ & $0.04 *$ & $0.04^{*}$ & $0.04 *$ & $0.04 *$ & $0.04 *$ \\
\hline \multirow[t]{2}{*}{ Patient 4} & BAS-GEXP & $0.04 *$ & $0.04 *$ & 0.19 & $0.04 *(\mathrm{sew})$ & $0.04^{*}$ (cycle) & $0.04 *$ (type) \\
\hline & BAS-FU & $0.04 *$ & $0.04 *$ & $0.04^{*}$ & $0.04^{*}$ & $0.04 *$ & $0.04 *$ \\
\hline \multicolumn{8}{|l|}{ MEXP } \\
\hline \multirow[t]{2}{*}{ Pat. 5} & BAS-GEXP & $0.04 *$ & 0.07 & 0.43 & $0.04 *$ (row) & 0.11 (climb) & 0.11 (type) \\
\hline & BAS-FU & $0.04^{*}$ & $0.04 *$ & $0.04^{*}$ & $0.04 *$ & 0.12 & $0.04 *$ \\
\hline \multirow[t]{2}{*}{ Pat. 6} & BAS-GEXP & $0.04 *$ & $0.04 *$ & 0.15 & $0.05^{*}$ (shopping) & $0.05^{*}$ (tinker) & $0.05^{*}($ clean $)$ \\
\hline & BAS-FU & $0.04 *$ & $0.04 *$ & 0.15 & $0.05^{*}$ & $0.05^{*}$ & $0.05^{*}$ \\
\hline \multirow[t]{2}{*}{ Pat. 7} & BAS-GEXP & 1.00 & 1.00 & 1.00 & 0.07 (clean) & $0.04 *$ (tinker) & $0.04 *($ cook $)$ \\
\hline & BAS-FU & 0.39 & 0.18 & $0.04^{*}$ & $0.04 *$ & $0.04 *$ & $0.04^{*}$ \\
\hline \multirow[t]{2}{*}{ Pat. 8} & BAS-GEXP & 1.00 & $0.04 *$ & 0.50 & 0.22 (shopping) & 0.25 (walk) & 0.22 (build) \\
\hline & BAS-FU & 1.00 & 1.00 & 0.39 & 0.11 & 0.11 & 0.11 \\
\hline
\end{tabular}

$* P<0.05$

${ }^{a}$ The intervention point was determined randomly

BAS, baseline; GEXP, graded exposure in vivo; FU, 6-month follow-up

Tables 3 and 4 also display the perceived harmfulness of the standard and specific activity at BAS and during the behavioral tests after GEXP and at FU. In line with the previous GEXP study in CRPS-I patients of De Jong et al. (2005a) these ratings were quite elevated. At the start of behavioral test 1 , the mean harmfulness ratings were lower than during BAS, suggesting that extinction during GEXP indeed generalized towards new activities. During behavioral test 1 the experienced harmfulness of the standard and specific activity was significantly lower in MEXP as compared to MSTI. Despite the observation that the activity was carried out without safety behaviors, particularly patient 3 (table 3 ) still experienced the fall forward as very threatening. The mean-score of the baseline-threat during behavioral test 2 in MEXP was for the standard activity almost and for the specific activity already decreased to zero. For these patients the 6-month period after behavioral test 1 was not followed by a (partial) return of perceived harmfulness. On top of that, activities that were rated as harmful during BAS and not used during the GEXP, were also rated as less harmful. By contrast, there was a larger reduction of perceived harmfulness in MSTI as compared to MEXP. 
Table 3 Overview of the performing of the standard activity (fall forward absorbed by the hands) during the behavioral tests after GEXP (behavioral test 1 ) and at FU (behavioral test 2) and the threat-score of the standard activity determined at BAS and during the behavioral tests for each patient in MSTI (GEXP: $\geq$ 15 activities) and MEXP (GEXP: 3 activities)

\begin{tabular}{|c|c|c|c|c|c|c|c|}
\hline \multirow[b]{3}{*}{ Condition } & \multicolumn{7}{|c|}{ Fall forward } \\
\hline & \multirow[b]{2}{*}{ BAS } & \multicolumn{3}{|c|}{ Behavioral Test 1} & \multicolumn{3}{|c|}{ Behavioral Test 2} \\
\hline & & $\begin{array}{c}\text { Baseline } \\
\text { threat }\end{array}$ & $\begin{array}{c}\text { Experienced } \\
\text { threat }\end{array}$ & Perform & $\begin{array}{l}\text { Baseline } \\
\text { threat }\end{array}$ & $\begin{array}{c}\text { Experienced } \\
\text { threat }\end{array}$ & Perform \\
\hline \multicolumn{8}{|l|}{$\overline{M S T I}$} \\
\hline Pat. 1 & 100 & 50 & 25 & $+/-$ & 50 & 30 & + \\
\hline Pat. 2 & 80 & 10 & 10 & + & 20 & 0 & + \\
\hline Pat. 3 & 100 & 70 & 70 & + & 50 & 40 & + \\
\hline Pat. 4 & 100 & 1 & 0 & + & 3 & 0 & + \\
\hline Mean & 95 & 33 & 26 & & 31 & 18 & \\
\hline$S D$ & 10.0 & 32.7 & 30.9 & & 23.3 & 20.6 & \\
\hline \multicolumn{8}{|l|}{ MEXP } \\
\hline Pat. 5 & 100 & 30 & 0 & + & 20 & 0 & + \\
\hline Pat. 6 & 100 & 10 & 0 & + & 0 & 0 & + \\
\hline Pat. 7 & 100 & 50 & 0 & + & 0 & 0 & + \\
\hline Pat. 8 & 100 & 30 & 10 & + & 0 & 0 & + \\
\hline Mean & 100 & 30 & 3 & & 5 & 0 & \\
\hline$S D$ & .0 & 16.3 & 5.0 & & 10.0 & .0 & \\
\hline
\end{tabular}

$+=$ performing activity without safety behavior, +/- = performing activity with safety behavior Range: 0-100 (the lower the score the better)

Bas, baseline of the study; GEXP, graded exposure in vivo; FU, 6-month follow-up

\section{Fear of movement/(re)injury}

Table 5 displays the mean TSK-score at BAS, after GEXP, and at FU for patients in MSTI and MEXP. The patients in MSTI (mean TSK-score: 42, SD = .8) and MEXP (mean TSK-score: $45, S D=6.7$ ) reported almost the same degree of pain-related fear at BAS. Both in MSTI (mean TSK-score: 26, SD = 4.4) and MEXP (mean TSK-score: 22, SD $=5.1$ ) pain-related fear decreased after GEXP. Based on the pre-set criteria, these results could be interpreted as clinically relevant. Despite the overall positive results of the TSK-score, MEXP (decrease of 23 points) showed a greater decrease than MSTI (decrease of 16 points). During FU the mean TSK-scores remained unchanged.

\section{The perceived harmfulness of daily activities}

The results of the PHODA are summarized in table 5. At BAS the mean total scores of the PHODA in Condition I (mean PHODA-score: 62) were higher than in Condition II (mean PHODA-score: 54). Both in Condition I (mean PHODA-score: 10) and Condition II (mean PHODA-score: 0.5) a relevant reduction in PHODA-scores was observed after GEXP. During FU the mean total score of the PHODA in Condition I (mean PHODA-score: 7) further decreased and remained almost at the same level in Condition II (mean PHODA-score: 0.3). Given that the PHODA consisted of 125 photographs and in both GEXP conditions patients were not exposed to all these activi- 
ties, it might be concluded that in both conditions extinction during GEXP generalized well to other activities.

Table 4 Overview of the performing of the specific activities during the behavioral tests after GEXP (behavioral test 1 ) and at FU (behavioral test 2) and the threat-score of the specific activities determined at BAS and during the behavioral tests for each patient in MSTI (GEXP: $\geq 15$ activities) and MEXP (GEXP: 3 activities)

\begin{tabular}{|c|c|c|c|c|c|c|c|c|}
\hline \multirow[b]{3}{*}{ Condition } & \multicolumn{8}{|c|}{ Specific Actvity } \\
\hline & \multicolumn{4}{|c|}{ Behavioral Test 1} & & \multicolumn{3}{|c|}{ Behavioral Test 2} \\
\hline & BAS & $\begin{array}{l}\text { Baseline } \\
\text { threat }\end{array}$ & $\begin{array}{l}\text { Experienced } \\
\text { threat }\end{array}$ & Perform & BAS & $\begin{array}{c}\text { Baseline } \\
\text { threat }\end{array}$ & $\begin{array}{c}\text { Experienced } \\
\text { threat }\end{array}$ & Perform \\
\hline \multicolumn{9}{|l|}{$\overline{M S T I}$} \\
\hline Pat. 1 & $100^{a}$ & $50^{\mathrm{a}}$ & $30^{\mathrm{a}}$ & + & $100^{d}$ & $65^{d}$ & $30^{d}$ & + \\
\hline Pat. 2 & $100^{b}$ & $10^{\mathrm{b}}$ & $0^{\mathrm{b}}$ & + & $100^{c}$ & $20^{c}$ & $0^{c}$ & + \\
\hline Pat. 3 & $100^{d}$ & $10^{d}$ & $10^{d}$ & + & $80^{b}$ & $50^{b}$ & $40^{b}$ & + \\
\hline Pat. 4 & $100^{d}$ & $1^{d}$ & $0^{d}$ & + & $100^{b}$ & $0^{b}$ & $0^{b}$ & + \\
\hline Mean & 100 & 18 & 10 & & 95 & 34 & 25 & \\
\hline$S D$ & .0 & 21.9 & 14.1 & & 10.0 & 29.3 & 28.9 & \\
\hline \multicolumn{9}{|l|}{ MEXP } \\
\hline Pat. 5 & $90^{c}$ & $20^{c}$ & $0^{c}$ & + & $90^{\mathrm{a}}$ & $0^{\mathrm{a}}$ & $0^{a}$ & + \\
\hline Pat. 6 & $100^{d}$ & $10^{\mathrm{d}}$ & $5^{d}$ & + & $100^{\mathrm{a}}$ & $0^{\mathrm{a}}$ & $0^{a}$ & + \\
\hline Pat. 7 & $100^{d}$ & $10^{d}$ & $0^{d}$ & + & $100^{c}$ & $0^{c}$ & $0^{c}$ & + \\
\hline Pat. 8 & $100^{\mathrm{a}}$ & $30^{\mathrm{a}}$ & $10^{\mathrm{a}}$ & + & $100^{d}$ & $0^{d}$ & $0^{d}$ & + \\
\hline Mean & 98 & 18 & 4 & & 98 & 0 & 0 & \\
\hline$S D$ & 5.0 & 9.6 & 4.8 & & 5.0 & .0 & .0 & \\
\hline
\end{tabular}

\section{Pain catastrophizing}

Regarding pain catastrophizing (table 5) the mean PSC-score at BAS in MSTI (mean PCS-score: 24.25, SD $=1.5$ ) was the same as in MEXP (mean PCS-score: 24.50, SD = 1.3). Both in MSTI (mean PCS-score: 6.5, SD = 5.1) and MEXP (mean PCS-score: 3.8, $S D=3.9)$ pain catastrophizing decreased after GEXP. Again, the decrease in MEXP (decrease of 19.2 points) was greater than in MSTI (decrease of 10.3 points). During FU the mean PCS-score in MSTI decreased still a little (mean PCS-score: 6.3, SD = 4.2). However, MEXP (mean PCS-score: 9.8, SD $=11.4$ ) showed an increase of pain catastrophizing during FU. 
Table 5 The mean total score of fear of movement/(re)injury (TSK), the perceived harmfulness of daily activities (PHODA), and pain catastrophizing (PCS), of MSTI (GEXP: $\geq 15$ activities) and MEXP (GEXP: 3 activities), determined at BAS, after GEXP and FU

\begin{tabular}{lcccc}
\hline & & & Mean total score & \\
& Condition & BAS & GEXP & FU \\
\hline TSK $^{1}$ & MSTI & 42 & 26 & 26 \\
& & $(\mathrm{SD}=.8)$ & $(\mathrm{SD}=4.4)$ & $(\mathrm{SD}=4.4)$ \\
& MEXP & 45 & 22 & 22 \\
& & $(\mathrm{SD}=6.7)$ & $(\mathrm{SD}=5.1)$ & $(\mathrm{SD}=5.1)$ \\
PHODA $^{2}$ & MSTI & 62 & 10 & 7 \\
& & $(\mathrm{SD}=2.6)$ & $(\mathrm{SD}=9.4)$ & $(\mathrm{SD}=6.8)$ \\
& MEXP & 54 & 0.5 & 0.3 \\
PCS $^{3}$ & MSTI & $(\mathrm{SD}=16.4)$ & $(\mathrm{SD}=1.0)$ & $(\mathrm{SD}=.5)$ \\
& & 24.25 & 6.5 & 6.3 \\
& MEXP & $(\mathrm{SD}=1.5)$ & $(\mathrm{SD}=5.1)$ & $(\mathrm{SD}=4.2)$ \\
& 24.50 & 3.8 & 9.8 \\
& $(\mathrm{SD}=1.3)$ & $(\mathrm{SD}=3.9)$ & $(\mathrm{SD}=11.4)$ \\
\hline
\end{tabular}

${ }^{1}$ Range: $17-68$ (the lower the better); ${ }^{2}$ Range: 0-100 (the lower the better); ${ }^{3}$ Range: 0-52 (the lower the better) ; Bas, baseline; GEXP, graded exposure in vivo; FU, 6-month follow-up

\section{Functional disability}

Table 6 displays the results of the RST and RASQ. The mean total score of the observed quality of the standardized activities of the RST determined at baseline were higher in Condition I. This means that patients in MSTI showed more objectively restrictions in the performance of ten daily life activities than the patients in MEXP. After GEXP, the mean total score decreased in both conditions. This was of the same size and clinically relevant. After GEXP no problems were observed by the assessor in MEXP. Despite a slight decline, this effect remained for all patients at FU. According to the results of the observed quality, patients in MSTI evaluated the standardized activities at BAS as more difficult than patients in MEXP. After GEXP the patients in MSTI evaluated the standardized activities of the RST as less difficult. In MEXP there were no difficulties reported. In both conditions the difference of the mean-scores was the same size. At FU there was no appreciable change in both conditions.

On the basis of the mean item scores of the RASQ it can be concluded that at BAS patients in MSTI indicated somewhat more disabilities than in MEXP. After GEXP, the mean item score in both conditions showed a clear and clinically important reduction of the reported disabilities. Compared to BAS the mean improvement in MSTI, calculated on the total number of items, was $53.3 \%$ (range: $43 \%-67 \%$ ) and in MEXP $61.5 \%$ (range: $48 \%$ - 75\%). During FU the mean item score of the RASQ was still significantly lower than at BAS. However, in both conditions these scores were 
higher than post treatment. In MSTI the mean relapse, once again calculated on the total number of items, was 5\%, and MEXP $21 \%$.

Table 6 The mean total score for the observed quality (observation) and subjective difficulties (evaluation) of the standardized activities of the Radboud Skills Test (RST), and the mean item score of the Radboud Skills Questionnaire (RASQ), of MSTI (GEXP: $\geq 15$ activities) and MEXP (GEXP: 3 activities), determined at BAS, after GEXP and FU

\begin{tabular}{lcccc}
\hline & & & Mean score & \\
& Condition & BAS & GEXP & FU \\
\hline RST observation $^{1}$ & MSTI & 17.3 & 5.5 & 6.3 \\
& & $(\mathrm{SD}=12.1)$ & $(\mathrm{SD}=4.8)$ & $(\mathrm{SD}=8.0)$ \\
& MEXP & 11.3 & 0.3 & 2.0 \\
RST evaluation $^{2}$ & MSTI & $(\mathrm{SD}=12.4)$ & $(\mathrm{SD}=.5)$ & $(\mathrm{SD}=3.4)$ \\
& & 8.5 & 2.8 & 2.3 \\
& MEXP & $(\mathrm{SD}=2.1)$ & $(\mathrm{SD}=1.9)$ & $(\mathrm{SD}=1.7)$ \\
& & 6.0 & 0.3 & 0 \\
RASQ $^{3}$ & MSTI & $(\mathrm{SD}=5.1)$ & $(\mathrm{SD}=.5)$ & $(\mathrm{SD}=0)$ \\
& 3.46 & 1.64 & 1.67 \\
& $\mathrm{MEXP}$ & $(\mathrm{SD}=.33)$ & $(\mathrm{SD}=0.51)$ & $(\mathrm{SD}=.43)$ \\
& 3.06 & 1.28 & 1.34 \\
& $(\mathrm{SD}=.79)$ & $(\mathrm{SD}=.42)$ & $(\mathrm{SD}=.53)$ \\
\hline
\end{tabular}

${ }^{1}$ The mean total score (sum of score for each single activity), Range: 0-40 (the lower the better); ${ }^{2}$ The mean total score (sum of score for each single activity), Range: 0-18 (the lower the better); ${ }^{3}$ The mean item score, Range: 0-5 (the lower the better); Bas, baseline; GEXP, graded exposure in vivo; FU, 6-month follow-up

\section{Discussion}

This study was designed to test the hypothesis that exposure to multiple stimuli promotes better generalization to new stimuli as compared to exposure to a limited number of stimuli. Using a randomized replicated single case experimental design, we decided to experimentally examine whether extinction of pain-related fear in patients with CRPS-I to the upper extremities generalized to threatening activities to which the patients were not exposed during GEXP. Two exposure conditions were compared, a condition during which patients were exposed once to 15 different activities (MSTI), and a condition during which patients were exposed five consecutive times to only 3 activities (MEXP). We hypothesized that generalization of extinction is facilitated in MSTI.

In contrast with our hypothesis, extinction of pain-related fear generalized equally well in both conditions. First, both forms of exposure resulted in a significant reduction in fear of movement, pain catastrophizing and functional disability. Second, despite pain reduction was not a direct goal, with the experience of less fear and the possibility to perform threatening activities even pain experience decreased in 
most patients. Third, behavioral tests after GEXP and at FU showed low perceived harmfulness for new activities that were rated as harmful during baseline, and to which patients were not exposed during exposure. Finally, the threat-value of daily activities presented by photographs (PHODA) decreased in all patients after GEXP and at FU patients still reported that these activities were not harmful.

In line with a previous exposure study in CRPS-I patients (De Jong et al., 2005a) the results of this study support GEXP as a successful treatment for CRPS-I patients reporting high levels of pain-related fear. After GEXP and during FU independent observations of daily activities showed that patients involved the affected hand to a full extent, whereas these were avoided at BAS. The success of GEXP is remarkable because pain is obviously central to the diagnosis of CRPS-I and pain reduction is not a direct goal of GEXP. Therefore, it seems that the catastrophic representation of pain is more important than the experienced pain intensity as it is. According to the theory of exposure patients learned during GEXP that the pain is less threatening than supposed, or were able to inhibit their fear responses. Using a motor imaginary task in CRPS-I patients Moseley et al. (2008) showed that pain catastrophizing and pain-related fear are not only related to change in pain but also to swelling which is an criteria of CRPS-I. The authors suggested that pain and swelling seems to be modulated by autonomic arousal and beliefs about pain.

Further, it is interesting to conclude that both exposure conditions facilitated generalization and overall somewhat better results are observed for MEXP. One possible explanation might be that during each exposure session all patients were exposed to a threatening activity which consists of several threatening movements. Recently, Rescorla (2006) suggested that greater extinction derives from the absence of the aversive stimulus in the presence of multiple compared to single original predictors of the aversive stimulus. Combined conditioned stimuli's (CS) for additional exposure sessions are superior to separate CS's, resulting in less spontaneous recovery, less re-instatement and slower re-acquisition (Rescorla, 2006). Based on findings on non-primate learning (e.g. Bouton \& Swartzentruber, 1991) and phobias (Rowe \& Craske, 1998a; 1998b), studies in chronic low back pain patients (Crombez et al.,1996; 1998; 2002; Goubert et al., 2002; 2005) already investigated whether exposure to different movements may enhance the generalization of the knowledge that 'many movements are not painful and hazardous'. However, no support was found for the hypothesis that varied exposure facilitates generalization of exposure effects. In contrast to the current study, generalization of exposure was tested whether the effects of one movement (e.g., bending forward, straight leg rising) generalized towards another dissimilar movement, whereas in this study patients were exposed to functional activities (e.g., garden, wash, vacuum, golf) which exist of several movements. Moreover, these activities were individually 
tailored based on a fear hierarchy. In the generalization studies of Crombez et al. (2002) and Goubert et al. $(2002,2005)$ the movements to which patients are exposed were selected by the researcher in question. The results of our study are more in line with the study by Trost et al. (2008) who showed successful generalization of pain expectancy corrections across movements in low back pain patients. These authors suggested that the increased intensity of performing tasks during the behavioral test is the reason that a gradual extinction of the over-predictive response was observed. In MEXP the increased intensity of the performing task mainly existed of duration and the number of repetitions of the three identified threatening activities, whereas in MSTI this was more the graded fear hierarchy of the activities. On the basis of this it can be assumed that not the variety of activities but the repeated exposure to some few individually identified threatening activities might be of importance.

Another different factor with regard to the above mentioned generalization studies is that, irrespective of the treatment condition, GEXP was provided to the maximum spectrum of patients' contexts and natural settings that can be achieved in and around the hospital setting. As Rescorla and Heth (1975) already suggested, to facilitate generalization it could be important that the UCS is the same as the UCS involved in the acquisition of the fear responses. In our study we used a behavioral test context that was different from the GEXP context and daily life situations. We defined the context in the behavioral tests in terms of activities (not addressed during GEXP and not presented in daily life), room (not used during GEXP) and time (after GEXP and during FU). The results showed clearly that the patients had a complete contextual control which caused overall generalization. Although patients were exposed to several movements, it seems that variation of the exposure context also help explain why both exposures are successful in the generalization of extinction.

Also, in direct contrast to the above-mentioned generalization studies we did not expect and strive for a fear reduction of $100 \%$ from baseline during one exposure session. Certainly during the first eight exposure sessions a fear-reduction of $50 \%$ was experienced as successful. This is in line with results of research on experimental manipulations which indicate that lower fear at completion of exposure does not predict better outcomes overall (Farchione, 2002; Rachman, Robinson \& Lopatha, 1987). Thus, whereas reported fear and dysfunctional beliefs generally declined within an exposure session (although not always), there is good evidence to indicate that this decline was indicative of learning or of long-lasting improvement.

A number of limitations to the present study must be considered. First, the preliminary results reported here are limited in that they are based on a small number of 
CRPS-I patients. Second, at BAS patients in Condition II had lower functional disability scores than those in Condition I. Despite randomization it appeared that patients in Condition II may have been less impaired. Further, the fact that both conditions differ in gender is an important shortcoming of the current study. Third, the effects of GEXP regarding generalization are examined in CRPS-I patients who reported high levels of fear of movement/(re)injury. However, pain-related fear is a general, overarching construct, encompassing various specific fears. For example, 'fear of interoceptive stimuli' like pain, skin color and temperature changes, and edema or 'fear of movement/(re)injury' which signifies the fear that certain movements may be harmful by causing (re)injury. Therefore, one should be cautious in generalizing to other patients until the effects of GEXP and the nature of pain-related fear are examined more extensively and their clinical implications are more fully considered. Fourth, despite some patients reported almost no pain after GEXP and during FU, overall the results showed and clinical experience has learned that performing some of the activities and/or movements still hurts. However, this not necessarily restricts daily life functioning. Nevertheless, it seems to be unlikely that a permanent extinction of anticipatory responses can be accomplished. Fifth, by use of a behavioral test, generalization was observed in a clinical setting and not in the home or work situation. Finally, the follow-up period may not have been sufficient to determine the long-term effect of GEXP in relation to generalization.

In sum, the current study supports that, irrespective of the kind of exposure, GEXP generalized to new threatening activities. Generalization was also found for observed quality of performance from standardized activities, self-reported functional disability, pain-related fear and pain catastrophizing, and the experienced pain and threat of daily activities. It seems that generalization of extinction is best facilitated if graded exposure exists of individually identified threatening activities existing of multiple movements to which the patient should be exposed several times. The mentioned results are of clinical importance as they suggest that under the influence of GEXP CRPS-I patients learn that pain is a common condition that can be selfmanaged, rather than as a serious disease or condition that needs careful protection. However, the results need to be verified in a wider (chronic) pain population. Besides, further research into the role of inhibitory processes during extinction and evocation of such processes at the time of re-exposure to a previously feared stimulus, and examination of the influence of context and time and fear tolerance is warranted. 

Chapter 7

\section{Summary and general discussion}


When traditional and biomedical treatments fail and pain-related disability grows, pain patients are often referred to rehabilitation centers. Generally, the treatment in these centers is interdisciplinary and based on bio-psychosocial or behavioral rehabilitation models which assume that pain disability is not only determined by the underlying pathology, but also, by cognitive, behavioral, emotional and social factors. Treatment methods from operant conditioning principles and psychophysiological and cognitive psychology concepts are applied to the treatment of chronic pain. Research shows variable results of cognitive-behavioral treatment programs. As a result, it is argued to match treatments to patient characteristics to increase the effectiveness of treatments (Turk, 1990). To develop effective customized treatments, in recent years behavioral pain research is focused on the mechanisms by which patients with acute pain become chronically disabled. A number of studies have reported that pain-related fear is one of the strongest predictors of variation in physical performance (Vlaeyen \& Linton, 2000).

Because of its substantial contribution of in the maintenance of chronic pain, painrelated fear is an important target for intervention. Indeed, several studies revealed that exposure in vivo (GEXP) is an effective treatment in chronic low back pain patients who report high levels of pain-related fear. However, on the basis of the results of these studies and the overall complexity of exposure there are still several questions that need to be addressed. For example, which treatment characteristics make the GEXP successful or unsuccessful in pain-related fear? What is actually learned during GEXP? Does the extinction of pain-related fear generalize to other movements and activities that were not included in the treatment? Is GEXP applicable in patients with pain disorders with observable tissue pathology such as neuropathic pain? In short, enough questions to answer in the process of optimizing GEXP in pain-related fear. By examining whether the effects of GEXP generalized from chronic low back pain to other pain problems such as complex regional pain syndrome and posttraumatic neck pain, the contribution of specific treatment components to outcome, and the question whether exposure to multiple stimuli enhances generalization after treatment, the goal of this dissertation was to further explore the utility of GEXP.

Before discussing the results, the main findings can be summarized as follows. The first study (chapter 2) focused on the role of pain-related fear in Complex Regional Pain Syndrome type I (CRPS-I). The results showed that pain-related fears, if measured with the PHODA, as well as pain severity were important predictors of pain disability. Although replications are necessary, the conclusion that pain-related fear in CRPS-I patients is a promising research direction in the understanding of pain disability in CRPS appeared justified. Chapters 3 and 4 described a number of singlecase experiments in which the effectiveness of GEXP in patients with posttraumatic 
neck pain (PTNP) and CRPS-I reporting substantial pain-related fear was tested. Both studies supported the effectiveness of a GEXP approach in these patients. GEXP was successful in decreasing not only pain-related fear, but also pain intensity, disability levels, and in increasing the daily physical activity level. Striking was also that physiological signs and symptoms related to CRPS-I were positively influenced by GEXP.

The reduction of pain-related fear occurred rather quickly after the initiation of the exposure treatment. As the exposure protocol used in the aforementioned studies always started with an educational part, the results might have been the result of the new information provided by the therapist during this initial session. Therefore, a study was carried out (Chapter 5 ) in which we examined the contribution of the educational part of GEXP in the reduction of pain-related fears and cognitions, and the associated disability and physical activity. The results showed that pain-related fears and cognitions were indeed positively influenced by the education. Contrary, performance measures of daily activities were not affected by the educational sessions and improved only during the exposure sessions. Finally, in chapter 6 it was examined whether the effects of GEXP in fearful CRPS-I patients generalized to threatening activities which were not addressed during GEXP. Overall, it seems that, irrespective of the kind of exposure, GEXP generalized to new threatening activities.

\section{Testing the role of pain-related fear in complex regional pain syndrome}

In recent years the investigation into potential pathophysiological mechanisms involved in Complex Regional Pain Syndrome type I (CRPS-I) has intensified. The general consensus is that the pathophysiology of CRPS-I is still not known (Wilson et al., 2005), and the contribution of psycho-physiological interactions to the development of CRPS-I is largely speculative. Although it has repeatedly been shown that in chronic musculoskeletal pain pain-related fear is associated with escape-avoidance behaviors and associated functional disability, the role of pain-related fear in CRPS-I was never tested systematically. Recently, Moseley et al. (2008) demonstrated that motor imagery increased pain and swelling in CRPS-I patients and that this effect seems to be modulated by pain catastrophizing and pain-related fear. According to the authors, these results corroborate the findings from recent studies that highlight the contribution of cortical mechanisms to pain on movement in CRPS-I which was suggested earlier (Andersen \& Buneo, 2003; Gieteling et al., 2008; Krause et al., 2006; Maihöfner et al., 2005, 2007; Schwenkreis et al., 2003). During movement, patients with chronic pain show more activation of the right insular cortex (Maihofner et al., 2007) thought to hold representations of the sympathetic nervous system (Hilz et al., 2006). The increase in swelling related to pain-related fear and pain 
catastrophizing suggests that cognitive variables may modulate the link between motor and sympathetic activities.

\section{Is pain-related fear an important predictor of general physical health and functional disability in CRPS-I?}

Pain-related fear is one of the strongest predictors of disability in chronic musculoskeletal pain and pain-related fear appears relevant CRPS-I as well. Therefore, we investigated the role of pain-related fear in CRPS-I using data of two cross-sectional studies (chapter 2). Study I included CRPS-I patients visiting an outpatient pain clinic. In study II, members of the Dutch association for CRPS-I patients were invited to complete a number of questionnaires. Overall, both studies showed that pain severity was an important predictor of general physical health and a higher level of functional disability respectively. Additionally, study I revealed a significant relationship between depression and general physical health. An interesting, and somewhat unexpected finding was that pain-related fear was an important predictor of functional disability in CRPS-I, but only when measured with a pictorial fear of activity scale (PHODA; Dubbers et al., 2003; Jelinek et al., 2003) -as was the case in study IIand not when measured with a more commonly used questionnaire tapping fear of movement/(re)injury (TSK, Miller et al., 1991). There are at least two explanations for this unexpected finding. One possible explanation for this could be that the PHODA, as opposed to the TSK, is more sensitive and better capable to capture inter-individual variation. The results show that the standard deviation of the mean TSK score is much smaller than that of the mean PHODA score. This means that it would be possible to infer that the patients have indicated that they believe pain due to movement is a sign of impending harm (TSK), but there is more individual variation in the kind of activities that are considered harmful (PHODA). Indeed, the PHODA was developed as a specific measure of pain-related fear, including pictorial presentations of concrete movements that are relevant for patients with upper and lower extremity pain. Conversely, the TSK is made up of statements reflecting the idea that painful movement in general is harmful. Since the PHODA requires patients to respond to specific pictorial stimuli reflecting movements of the upper or lower extremities, it has also the potential to activate fear schemata more directly and elicit emotional reactions. The importance of the immediate and specificity of fear provoking stimuli has been noted by several studies. For example, Lang (1995) showed reliable affective psychophysiologic responses, defined by the judged valence (appetitive/pleasant or aversive/unpleasant) and arousal elicited by a threatening pictures. Picture-evoked affective responses are also associated with potentiated startle reflexes during unpleasant pictures and inhibited startle reflexes during the presentation of pleasant pictures. In addition, both effects were augmented by 
high picture arousal. Also Wessa et al. (2005) showed in a group of motor vehicle accident survivors with subclinical posttraumatic stress disorder (PTSD) that accident-related pictures enhanced startle responses and neutral pictures had a significantly lower response. The authors suggest that traumatized persons with PTSD show exaggerated emotional responses to trauma-related stimuli and reduced cognitive responses to several types of stimuli that may interfere with the extinction of the emotional trauma memory. It would be interesting to study psychophysiological responses of CRPS patients when exposed to the PHODA pictures. Another explanation is that PHODA measures expected pain level fear of pain in certain contexts. Contrary to the findings in patients with low back and cervical pain (e.g. Vlaeyen \& Linton, 2000; Nederhand et al., 2006), clinical experience suggests that CRPS-I patients describe their fear not in terms of movement and injury, as tapped with the TSK, but in terms of uncontrollable interoceptive stimuli (e.g. pain). Previous research (e.g. Asmundson \& Nicholas-Carleton, 2005; Keogh et al., 2001; Carleton et al., 2006) has shown that fear of pain, as measured with PASS, increases vulnerability to pain disability. However, as far as known, it is never examined whether fear of pain is related to CRPS-I. An intriguing question is whether pain-related fear in neuropathic pain has different features than in musculoskeletal pain, or more specifically what the exact conditioned stimulus is. It might well be that in CRPS patients aversive interoceptive stimuli are more important than proprioceptive ones which may be more relevant to chronic back pain patients.

\section{Exposure in patients with Posttraumatic Neck Pain and Complex Regional Pain Syndrome type I reporting substantial pain-related fear}

Overall, the general understanding is that Posttraumatic Neck Pain (PTNP) and Complex Regional Pain Syndrome type I (CRPS-I) are complex in nature and that the number of empirically supported treatments for these pain disorders remains limited. Nevertheless, in chronic pain management there is increasing consensus regarding the importance of a behavioral medicine approach to symptoms and disability. For patients with PTNP, cognitive behavior therapy (CBT) programs have been developed as well, of which those promoting physical activity and are focussed on regaining function have proven to be relatively more effective (Hurwitz et al., 2008). Although these studies suggest that activity increase is associated with faster return to work and a decrease in pain and disability levels, there is evidence showing that these changes are mediated by the reduction of the catastrophic (mis)interpretations of pain (Smeets et al., 2006, Leeuw et al., 2008, Spinhoven et al., 2004). In CRPS-I functional restoration and reactivation via interdisciplinary approach have been thought to be required for optimal outcome (Harden, 2005). However, the specifics as to what the components of an effective, efficient functional restoration 
program should be, what modalities are to be delivered, when, and for how long were all open questions.

Given the beneficial results of GEXP in CLBP patients who report high levels of painrelated fear, and because pain-related fear has shown to be associated with PTNP and CRPS-I, there are good reasons to believe that a GEXP treatment would be beneficial for fearful PTNP and CRPS-I patients as well. For the majority of these patients explanations grounded in physical pathology are inadequate and conventional approaches to pain have failed, which suggest that alternative explanations need to be explored. Patterns of pain-related avoidance behaviour seen among these patients can be individually analyzed using an associative fear learning framework and subsequently targeted with exposure-based treatments. From this perspective, the mechanism of action for successful treatments in fearful PTNP and CRPS-I patients is based on a frequent and gradual increase of pain-eliciting stimuli without trying to reduce or alter the experience. Indeed, the exposure to movements might correct the sustained mismatch between motor activity en sensory feedback in PTNP and CRPS-I patients (e.g. Harden, 2005, Nijs et al., 2009), which may serve as ongoing source of nociception. The incorporation of GEXP as therapeutic approach for functional restoration is then axiomatic, but should be confirmed.

\section{Does GEXP extend to PTNP and CRPS-I patients?}

To answer this question, two intervention studies were conducted. The current studies supported a GEXP approach to chronic PTNP (study I) and CRPS-I (study II) patients reporting elevated levels of pain-related fear. The GEXP was successful, and as shown in study I, superior to GA in decreasing levels of pain-related fear, pain catastrophizing, pain disability, and pain intensity. In the same study also improvements were found in the performance of physical activity in the home situation as measured with ambulatory activity monitors. A remarkable finding was that the participants of Study II reported that they did not experience any physiological signs and symptoms anymore after GEXP and even at 6 month. However, it is not clear how GEXP might address either of these processes. One possible explanation is that GEXP is a strategy mechanism that impacts higher order motor processes such as motor intent or motor planning. Another explanation might be that GEXP focused on mental and visual attention in order to move the affected extremity. Thus, perhaps GEXP requires the patient to attend to the affected extremity, which may serve to reverse a learned disuse of the affected extremity (Butler, 2001).

Despite the success of GEXP several limitations of these replicated single case experimental studies should be mentioned. First, the preliminary results reported are 
limited in that they are based on a small number of patients. However, in both studies an experimental design was chosen with appropriate statistical analyses (randomization tests). Randomization tests have the advantage of being valid for singlecase experiments without making distributional assumptions, of being easy to apply, and of being extremely versatile for even the most complex single-case designs (e.g. Onghena \& Edgington, 2005). Conversely, the results of the non-daily measures, with limited number of data points, were not subjected to randomization tests and for the most part based on arbitrarily chosen preset-criteria. Second, only in study I GEXP was compared to another intervention. Because the experimental design did not include washout periods between the different treatment components - for evident reasons-, it is possible that carry-over effects occurred. Indeed, when GA followed GEXP the improvements remained stable, which is also consistent with the favourable FU results. As already noted, GA showed to be helpful for chronic neck pain disability across a number of studies. However, in study I, change during GA was marginal at best, when GA preceded GEXP. Both GA and GEXP were performed according to a specific protocol. In addition, GA and GEXP were delivered by different therapists. Because patients' attitudes and beliefs, and thereby patients' disability levels, may be derived from the projected attitudes and beliefs of health care providers (e.g. Rainville, 1990), the two teams were comparable in terms of experience and therapists' preferences. Efforts were made to achieve the same level of enthusiasm in each therapist who participated in either GA or GEXP. Nevertheless, therapists may consider GEXP as a more credible treatment than GA which may have influenced their treatment behaviour in favour of GEXP (e.g. Leeuw et al., 2008). Third, study II was impeded by the use of self-report measures only, which may have been subject to response bias. Finally, the 6-month FU may not have been sufficient to determine the long-term effect of GEXP or long-term quality of life. To increase generalization of GEXP across patients more simultaneous or sequential replications of single-case experiments with future measurement occasions are needed. Replication strategies also allow one to design small clinical trials, detect patterns of response or subgroup of patients or design Randomized Clinical Trials (RCT) as a collection of replicated single-case experiments. Further, physiological measures (e.g. pain thresholds, oedema and thermal detection) or objective measures of behavior seem most promising as target measures in single-case experiments for pain research. 


\section{How many exposure sessions are needed to demonstrate trend changes and significant effects?}

GEXP has already successfully been applied in chronic low back pain (CLBP) patients. Not only a number of single-case experimental studies have been carried out successfully, also randomized controlled clinical trials have shown positive results with regard to pain disability and pain-related fear (Leeuw et al., 2008; Linton et al., 2008; Woods \& Asmundson, 2008). However, in comparison with the single-case experimental exposure studies in patients with low back pain of Vlaeyen et al. (2001; 2002a; 2002b) the current studies showed that more exposure sessions were needed to demonstrate trend changes and significant effects in PTNP and CRPS-I patients. A possible explanation is that PTNP and CRPS-I patients experience multiple complaints and fears, not just fear of movement. Not only neck pain but also symptoms such as headache, visual disturbances, dizziness, weakness, paraesthesia, nausea, both upper and lower limb numbness and tingling, tinnitus, and cognitive problems (concentration and memory disturbances) are common in PTNP patients in the acute stage after a traumatic event (Barnsley et al., 1994; Ferrari et al., 2005). Symptoms of CRPS-I can also include abnormal swelling, abnormal hair or nail growth, abnormal skin color or temperature changes, abnormal sweating, limited range of motion and movement disorders (Wilson et al., 2005). Although pain is normally a leading symptom in CRPS-I, the autonomic, motor, and trophic signs and symptoms of CRPS-I are not necessarily coupled with pain (Eisenberg \& Melamed, 2003). In many patients with CLBP, the main concern is the experienced pain interfering with daily life activities signals injury. These concerns can relatively easily be challenged as injury will never follow increased physical activity, given the nonspecificity of their pain complaints. In contrast, the concerns of PTNP patients (e.g. "If I would lift heavy weights, then I do not have full control of my neck, which will worsen the pain complaints, with the result that I will not be able to do my job in the future") and CRPS-I patients (e.g. "If I would walk without crutches, the pain will worsen and the foot becomes thick and intensely hot, by which I will loss control of my leg, which increased the likelihood of uncontrolled movements, which will aggravate the CRPS") may be much more difficult to challenge. Another explanation of the delayed effect could be that, contrary to GEXP in CLBP, the sessions mainly consisted of exposure to personally relevant activities that represented the main functional goals that were chosen by the patients themselves occurred in a later phase. Because of the multiple fears in PTNP and CRPS-I patients, the progress in the fear hierarchy occurred more slowly than during GEXP in CLBP.

In CRPS-I patients it is possible that the observed peripheral changes of CRPS-I mediated the effect of information on pain. This assumption is supported by a study of Arntz and Claassens (2004) who tested whether the meaning attached to pain influ- 
ences the pain intensity. By suggesting that a very cold metal bar which was briefly placed in the neck was either hot or cold, the potentially harmful property of the stimulus was experimentally manipulated. Participants who were given the information that the metal bar was hot reported more pain than those who were told that a cold bar was applied to their neck. The authors found that harm beliefs are a crucial aspect of meaning that mediated the association between the information and provided report of pain. Based on these findings it seems interesting to investigate whether there is a difference between CRPS-I patients who experience there vasomotor abnormalities in the affected extremity as warm or those who experience them as cold. In CLBP patients, possible tissue-damage is not observable, by which it might be easier to modify their meaning attached to their pain as compared to CRPS-I patients in whom symptoms are clearly visible. Additionally, these observable symptoms might be interpreted catastrophically and may direct hypervigilance to sensations of the body, thereby amplifying the pain experience. Indeed, there is evidence that the intensity of pain is related to this vigilance (e.g. Crombez et al., 2004). In line with this the catastrophic interpretation of pain might include anxiety, which in turn might amplify pain intensity (Arntz et al., 1991; 1994; Arntz \& de Jong, 1993).

\section{Mediating effect of GEXP}

According to the single-case studies of Vlaeyen et al. (2001; 2002a; 2002b) it is of interest that not only pain-related fear, pain catastrophizing and pain disability but also pain experience is positively affected by GEXP. Moreover, the results suggest that the decrease in pain experience temporarily follows an associated decrease in pain-related fear. This is in line with the Fear-Avoidance model. However, such strong reduction in pain is not common in usual cognitive-behavioral treatments for chronic pain (Kole-Snijders et al., 1999; Morley et al., 1999). Furthermore, it is remarkable that in study II self-reported trophic changes or enhanced efficacy of nociceptive mechanism or cortical changes are influenced by GEXP. We offer five possible explanations for these findings.

First, one explanation is that change in pain and autonomic, motor, and trophic signs and symptoms of CRPS-I are related to pain catastrophizing and pain-related fear which are the primary focus of GEXP. Indeed, Moseley et al. (2008) found in patients with CRPS-I and also in a group of non-CRPS pain that the higher patients report pain-related fear or pain catastrophizing, the larger the increase in pain and swelling with imagined hand movements. Sympathetic arousal is positively related to an increase of swelling. Maihöfner et al. (2007) showed that during movement chronic pain patients have more activation of the primary insular cortex, thought to hold representations of the sympathetic nervous system, than healthy controls. The 
findings that increase in swelling are related to pain-related fear and pain catastrophizing suggests that cognitive variables may modulate the link between motor and sympathetic activities. The results of Moseley et al. (2008) suggest that pain-related fear affects motor processes and pain even in the absence of actual performance of physical movement, also demonstrating that cognitions may modulate pain processing in the absence of nociception.

Second, it is demonstrated that some chronic pain patients have an involuntary neurological neglect-like condition (Galer \& Jensen, 1999; Förderreuther et al., 2004; Frettlöh et al., 2006). This finding suggest that PTNP and CRPS-I patients may experience a change in their body perception which can be very unsettling, frightening and/or repulsive, and ensures avoidance behavior. Therefore, for the patients it could be difficult to maintain conscious attention to the affected limb and its adequate use, which will consequently worsen the disordered sensorimotor function. Thus, the success of GEXP may be due to requiring to attend to the affected extremity for a certain time. In which case, GEXP may simply serve to reverse a learned experiential avoidance response of the affected extremity or limb.

Third, the reduction of pain-related fear levels may be due to a reduction of selective attention towards the affected body extremity. Experimental studies on the role of attention and pain-related fear have shown that chronic pain patients with elevated levels of pain-related fear habitually attend to somatic sensations (Asmundson et al., 1997; Peters et al., 2002). These findings suggest the decrease in pain experience during GEXP was mediated by a process in which the reduction of the threat value of previously fear-eliciting stimuli also produced a redirection of the attention away from pain and bodily sensations.

Fourth, the effects of GEXP could also be mediated by an increase in acceptance of pain. For example, Wicksell et al. (2008) tested an intervention consisted of valuesbased exposure and acceptance strategies to improve functioning and life satisfaction by increasing the participants' abilities to behave in accordance with values in the presence of interfering pain and distress (psychological flexibility). In this study, the functional relationship between chronic pain and disability was explained by a learning theory model emphasizing avoidance of unpleasant experiences (e.g. pain, fatigue, fear; Fordyce, 1976). For chronic pain patients activities that reduce pain and distress but are less active and stimulating will likely produce a reinforcing short-term relief. Over time, this behavior may gradually compromise functioning and life quality without a corresponding decrease in symptoms. Exposure to previously avoided situations is considered to be the core intervention, emphasizing acceptance of what cannot be directly changed (thoughts, emotions, bodily sensations) as a way of engaging in activities that are meaningful but possibly painful or 
fear-provoking. Behavioral activation was not carried out during sessions but rather by the patients between sessions. The goal of acceptance and commitment therapy (ACT), is to increase psychological flexibility, defined as the ability to act effectively in accordance with personal values in the presence of negative private experiences such as pain or distress (Hayes et al., 2006). Compared to a wait-list control condition Wicksell et al. (2008) showed that after the exposure-ACT condition for pain disability, life satisfaction, fear of movements, depression and psychological inflexibility significant differences in favour of the treatment group were seen. For both groups no change was seen in pain intensity, which implies that reported increase in functional ability and life quality was not due to a corresponding decrease in pain. Based on these results and because GEXP differs from the exposure strategies as applied by Wicksell et al. (2008), future GEXP studies may test the validity of the idea that the results are mediated by increased acceptance.

Fifth, in the literature on catastrophizing's influence on pain in the context of rheumatic disease (RA) it is suggested that pain catastrophizing might be directly or indirectly associated with inflammatory processes (Edwards et al., 2006). For example, Schoenfeld-Smith et al. (1996) have reported positive relationships between catastrophizing (or helplessness, one component of catastrophizing) and elevated indices of disease activity and inflammation. Because studies in RA have shown that high levels of catastrophizing prospectively predicted worsening erythrocyte sedimentation rates (e.g. Evers et al., 2003), these authors suggest directional relationships between pain catastrophizing and inflammation. Catastrophizing may also contribute to interactions between pain and the immune system (Watkins \& Maier, 2002). In sum, although no blood samples were taken from our patients, one could speculate that effects of GEXP might be mediated by a reduction of pro-inflammatory cytokines associated with a reduction of pain catastrophizing.

\section{GEXP initially increases pain levels}

Despite the overall positive influence of GEXP on pain experience both in study I and II there appears to be an increase in pain experience at the onset of GEXP. In the PTNP-study a possible explanation may have to do with the natural history of the participants. They could be characterized as not-at-fault drivers who experience feelings of are anger at the other driver who caused the injury and keeping him/her from attaining an important life goals (Ferrari et al., 2005; Mayou, 1994; Berkowitz \& Harmon-Jones, 2004). High levels of anger might be associated with more intense pain experience and more severe disability (Bruehl et al., 2003; Burns \& Bruehl, 2005). It has been suggested that perceptions of injustice and anger are related. Sullivan et al. (2008) found that individuals who perceived injustice and had sustained injuries in motor vehicle accidents, as a result of someone else's fault, also 
reported more severe pain, more pain sites, and greater depression. In addition, DeGood and Kierman (1996) found that chronic pain patients who blamed others for their pain reported more pain and more emotional distress than patients who did not ascribe fault for their pain condition. It is interesting to note that research suggested that blaming others for one's situation appears to be sufficient to engender negative outcomes (Turk \& Okifuji, 1996; Blyth et al., 2003) and that perceptions of injustice might allow individuals to remain stuck in their current pain situation. Also subjectively aversive conditions which are the result of the injury can generate anger (Berkowitz \& Harmon-Jones, 2004). An example of such aversive conditions could be exposure to activities and/or movements in which physical discomfort or pain will be experienced (Anderson et al., 1998; Berkowitz \& Harmon-Jones, 2004). The induction of anger and pain-evoked cardiac responses that are elicited by anger produces increased pain intensity and pain unpleasantness (Rainville et al., 1995). However, there are also studies that have shown the opposite, namely that anger just inhibits pain (e.g. Bruehl et al., 2009). Despite the negative role of anger and perceived injustice on pain experience at the start of GEXP, data from the GEXP study in PTNP (see this thesis) indicate that exposure therapy finally helps reduce the hallmark features of chronic PTNP (e.g. pain experience, disability and fear).

\section{Moderation}

Besides the specific effects of exposure it is important to consider the influence of non-specific effects as well. (e.g. Mahomed et al., 2002; Vase et al., 2005; Goffaux et al., 2007). Overall, positive or negative expectations may result in the reduction or amplification of pain along with inhibition or activation of several brain regions (Sawamoto et al., 2000; Porro et al., 2003; Lorenz et al., 2005; Keltner et al., 2006). According to these observations it seems that expectation of either low- or highintensity painful stimuli has a strong influence on the perceived pain. Clinical studies on the effectiveness of cognitive-behavioral rehabilitation programs for chronic low back pain and fibromyalgia showed that patients' initial beliefs about the success of a given pain treatment have an important influence on the final treatment outcome, and that patients with higher treatment expectancies significantly received less disability compensation, were less fearful and more satisfied (Goossens et al., 2005; Smeets et al., 2008).

A criticism of study I is a lack of consideration for the influence of non-specific effects such as expectation. On the other hand, in study II patients were asked for expectancy and credibility of GEXP. This was done after the educational part of the GEXP, and before the actual exposure. Participants not only received the rationale of the exposure therapy and a positive approach of the diagnosis CRPS-I, a verbal suggestion that clinical improvement should be expected was given as well. Inde- 
pendently, both the rehabilitation physician and the other team members told the patients that they may expect a positive influence of GEXP on their pain complaints. The mean expectancy and credibility ratings were relative low for the GEXP in study II. This implied that the patients did not expect that GEXP will help to cope better with their pain complaints, and that they did not believe that GEXP was a meaningful treatment for patients with CRPS-I. However, given the medical history of the CRPS-I patients who have participated in this study, this negative expectancy is hardly surprising. Overall, chronic CRPS-I patients are usually confronted with repeated failures finding a solution for their pain problem. Contrary to be expected, the low expectancy about GEXP did not had any negative effect on the experienced clinical benefits immediately after GEXP and at 6-month FU. All patients did not only report a large decrease in pain disability but also in pain experience and CRPS-I related physiological signs and symptoms.

Finally, effectiveness is not a feature of exposure alone. In the first place, the interactions between patient, therapist and/or therapist team and the exposure sessions and/or therapy program are important for the success of GEXP. For example therapeutic alliance, motivation of the patient, therapist/team prognoses, therapist appraisal of patients behavior, attitude of the therapist (self-confidence, no concern/anxiety when patient bellow with pain) or planning of the sessions are just a few examples which could define the course of the GEXP. As an addition to the ongoing discussion concerning the magnitude of therapist effect in GEXP, therapist variables appear to influence treatment alliance more than outcome (Wampold, 2001).

\section{Contribution of specific treatment components to outcome and generalization of GEXP}

\section{Education versus GEXP}

The results of the first single-case experimental studies examining the effectiveness of GEXP in patients with chronic low back pain (CLBP) showed abrupt changes in self-reported pain-related fears and cognitions (Vlaeyen et al. 2001, 2002a, 2002b). These changes seem to be more characteristics of insight learning rather than the usual gradual progression of trial and error learning. The educational session at the start of GEXP might have contributed to this insight. To test this hypothesis it was examined in six consecutive CLBP patients, whether a single educational session followed by GEXP or operant graded activity (GA) had indeed a powerful treatment effect. Both daily measures and pre-post assessments showed that after education and the following no-treatment period, subjective ratings of pain-related fears and 
catastrophizing decreased substantially. Based on these results it seems that a tailor-made formulation of a patient's pain problem, within a substantive fearavoidance model, has good empirical support in patient's perceptions about the harmfulness of physical activity and threat value of pain. Moreover, in reducing pain-related fear the results suggest that the education of the current study works in one session which implicates that it would be a really effective intervention to use in primary care if one could identify at-risk patients. Except providing personalized information the aim of the education was also that patients should indicate their adherence to participate in major behavioral change in order to regulate themselves in the period ahead. Adherence implied the patient's active choice in the process, rather than passive co-operation or obedience to the recommendations of the therapist team. Further, it was a process that therapists and patient undertook in order to reach an agreed plan of disability management. Finally, by inviting family or close friends of patients during the educational session the therapist team tried to realize social and emotional support which also appeared to lead to lower levels of anxiety (Edwards \& Clarke, 2004).

In addition, the current study has shown that education, although essential, is not sufficient to guarantee that patients actually perform fearful activities and/or movements. Educating the patient that being active leads to functional possibilities alone, and perhaps even a better quality of life, is not enough to make him/her more active. Self-reported performance in daily life, subjective ratings of the PHODA and even activity monitor data support the assumption that GEXP is necessary to modify escape and avoidance behavior due to pain-related fear and not GA. It seems that fearful chronic pain patients can change their behavior by means of exposure therapy. However, motivation to change behavior requires active participation on their part.

A possible reason for the lack of association between beliefs and behavior is that the willingness to perform health-related behaviors involves a complex tangle of attitudes to physical activity, implicit versus explicit affective evaluation, expectations, previous experiences of physical activity, and social pressure. Despite decision alternatives available in memory, decisions often rely on an "attitude-based" strategy, thereby ignoring available and contradictory information (Sanbonmatsu \& Fazio, 1990). This means that attitudes such as the deeply ingrained pain-related fear may evoke automatic activation from memory when the patient encounters threatening situations. Explicit attitudes are distinguished from implicit or automatic attitudes and both may guide one's behavior (Fazio, 1990). Explicit attitudes may guide one's behavior by a deliberate and conscious analysis of the costs and benefits of that behavior, whereas implicit attitudes may guide in a more spontaneous and affective manner, without actively considering the positive and negative as- 
pects. This means that patients may have different evaluations of the same activity. For example, the patient realizes that his concerns about harm are irrational; however, when suddenly confronted with a threatening movement avoidance of movement will occur all the same. The results of the current study suggest that education did not have a strong effect on patients' implicit attitudes toward pain-related activities or movements but did change their explicit attitudes. It seems that explicit attitudes toward pain-related activities or movements are readily changed when faced with the fear-avoidance model that is inconsistent with the existing propositions about pain. On the other hand, implicit attitudes may change only when new associations are formed through evaluative conditioning or when new propositions ingrain in into new associations (Gawronski \& Bodenhausen, 2006). Based on the results it can be assumed that GEXP offers sufficient possibilities for evaluative conditioning processes to change implicit attitudes toward pain-related activities or movements. In addition, because changes of implicit attitudes toward pain-related activities or movements, which are mediated by changes in cognitive strategies (i.e. more positive propositions about pain), may become apparent if there is enough time to ingrain these propositions, it seems that GEXP allows for this possibility.

\section{Generalization}

Despite the success of GEXP in chronic pain patients who report high levels of painrelated fear, it is still a question whether fear extinction generalizes to new threatening activities. The results of the activity monitor data of the PTNP-study and the education-study suggests that the confrontation of fear-eliciting activities in the treatment setting is analogue for how patients respond in daily life situations. Moreover, treatment gains produced during GEXP seems to generalize to the home setting and in the absence of therapists. Contrary, some recent experimental studies raise doubt about the generalization of the exposure effects (e.g. Vansteenwegen et al., 2007; Goubert et al., 2005). Research into the return of fear has revealed that extinction does not mean unlearning (e.g. Craske et al., 2008). It appears that the extinguished fear response can arise spontaneously after a while (spontaneous recovery), or reoccur when confronted with a new CS-US event (rapid reacquisition) or an unpredicted US (reinstatement). In addition, it also appears that extinction is context-sensitive (e.g. Craske et al., 2008). It has been established that what has been learned during the process of extinction not necessarily generalizes towards other situations; not even when the extinction process takes place during the original process of acquisition.

Whenever context changes can result in return of fear, the subsequent question is how this return can be prevented and on top of that which typical feature of GEXP is responsible for the supposed generalization. Animal research showed that when- 
ever extinction is received in different contexts it leads to less return of conditioned suppression in yet a new context when compared extinction in only one context (Chelonis et al., 1999; Gunther et al., 1998). In human demonstration of multiple context methods as prevention for return of fear is still scarce. By the use of computer-conditioned suppression tasks Neumann (2006) demonstrated that extinction in multiple contexts can reduce renewal of fear. Further, using retrieval cue methods Vansteenwegen et al. (2006) and Collins and Brandon (2002) showed larger return of fear when cues were given that were also present during acquisition than when retrieval cues were also present during extinction. Furthermore, Rowe and Craske (1998) showed that return of fear towards a new spider exemplar was reduced when multiple stimulus examples were used during exposure instead of one and the same spider. Finally, Vansteenwegen et al. 2007 showed that spider-anxious students that were exposed to videotapes with the spider presented in different locations of a house showed less return of fear when confronted with the spider in a new context than students that were exposed to repeated presentations of a videotaped spider in one specific location of the house.

In this thesis a clinical study is described in which the primary objective was to replicate the finding that exposure to multiple conditioned stimuli can enhance the generalization process of extinction. By the use of a randomized replicated single case experimental design it was examined whether GEXP generalized better to a new movement when fearful CRPS-I patients were exposed to 15 activities once as compared to an GEXP program in which fearful CRPS-I patients were exposed to 3 activities to which patients were five time each. In contrast to the expectations, both conditions generalized to new threatening activities. Based on these results it seems that multiple stimuli exposure is not a sine qua non to prevent relapse.

Independent of the context in which certain activities and/or movements will be performed it seems that the experienced pain and even more the catastrophic representation of pain is important in CRPS-I patients. According to the theory of extinction CRPS-I patients learned during GEXP that the US (pain) is less threatening than supposed. The US was, as it were, biased by the representation. In addition, it seems reasonable that the supplemented cognitive techniques during GEXP were necessary to directly challenge negative valence and also the validity of catastrophic assumptions and misinterpretations by which generalization can be realized.

Further, a possible explanation of the success of generalization might be that in both GEXP conditions during each exposure session all patients were exposed to a certain threatening activity which consists of several threatening movements. Moreover, these activities were individually tailored based on a fear hierarchy. It can be assumed that not the variety activities but the repeated exposure to some few indi- 
vidually identified threatening activities might be of importance. Another explanation could be that the CS was not the movement or activity, but the painful sensation itself. This may mean that it does not matter which movements or activities are identified, but that exposure to pain and related physiological sensations is sufficient to ensure generalization. In this case, GEXP seems to be a form of interoceptive exposure (e.g. Flink et al., 2009).

Finally, to facilitate generalization it could be important that during GEXP the CS is the same as the CS involved in the acquisition of the fear responses. GEXP was provided to the maximum spectrum of patients' contexts and natural settings that can be achieved in and around the hospital setting. Although patients were exposed to several movements, it seems that variation of the exposure context also help explain why both exposures are successful in the generalization of extinction. Finally, based on results of research on experimental manipulations which indicate that lower fear at completion of exposure does not predict better outcomes overall (e.g. Farchione, 2002), the protocol of GEXP does not strive for a fear reduction of $100 \%$ from baseline during one exposure session. Certainly during the first eight exposure sessions a fear-reduction of $50 \%$ was experienced as successful. Thus, whereas reported fear and dysfunctional beliefs generally declined within an exposure session (although not always), there is good evidence to indicate that this decline was indicative of learning or of long-lasting improvement.

\section{Where do we go from here?}

Despite the success of GEXP in chronic pain patients who report high levels of painrelated fear there is still uncertainty about the basic principles that underlie it. Therefore, there is a need for more theory-based research. Studies focusing on expectancies, context, and neurocognitive processes might shed more light on the mechanisms underlying associations between pain-related fear and chronic pain.

\section{Expectancies}

Expectancies for the likelihood of aversive events are central to human fear condition (e.g. Craske et al., 2008). In chronic pain for example, propositional knowledge about the association between two stimuli, such as a specific activity and/or movement (CS) and subsequent pain or pain increase (US), as a potential sign of injury, (e.g. Lovibond \& Shanks, 2002; Ohman \& Mineka, 2001), is considered a strong correlate of conditioned responding. As already suggested, expected occurrence of the US (pain) is violated during GEXP. During GEXP, a mismatch between the expectancy of negative consequences of a certain activity and/or movement and the 
absence of these consequences occurs. There probably are a number of ways of violating expectancies for pain (US). In the view of the studies in this thesis it seems that this may extend to the role of cognitive techniques applied within GEXP, which aim to shift expectancies for aversive events through logical empiricism. Future research should explicitly examine the role of cognitive techniques and which misconceptions are corrected during GEXP in order to fully understand what has been learned or has not been learned. Furthermore, research to durations or frequencies of unreinforced exposure to certain activities and/or movements that surpass the rate at which aversive events are expected to occur is warranted. It might be worthwhile to manipulate the duration of the GEXP trials relative to violation of fear-based expectancies.

\section{Safety behavior}

Should we wean safety signals and safety behaviors during GEXP? A number of studies have supported the hypothesis that safety behaviours are interfering with the benefits of exposure therapy (e.g., Sloan \& Telch, 2002). Hence, by relying on safety behavior, anxious pain patients might be unable to obtain disconfirmatory evidence related to their unrealistic beliefs about certain pain stimuli. Indeed, they might conclude that their own actions (i.e., the safety behavior itself) prevent feared outcomes. In the context of GEXP, such strategies might thus inhibit the process of adaptive cognitive change about pain and related physiological signals. Tang and colleagues (2007) were the first who examined the use of safety-seeking behaviors (SSBs) in chronic low back pain patients. By exposing participants to pain-provoking situations (such as lifting) these authors identified a wide range of SSBs, particularly in patients with high health anxiety, which continued throughout the time the patient remained in a feared situation. Based on their findings Tang et al. (2007) suggested that SSB is distinct from overt pain behavior and may be a defining characteristic of chronic pain patients reporting high levels of health anxiety. Further, the study of Tang et al. (2007) also showed that the use of SSB was more strongly correlated with the presence of catastrophic thoughts during pain-provoking tasks, whereas the display of overt pain behavior was more strongly correlated with the level of pain experienced during these tasks. It is hypothesized that pain patients who catastrophize about physical activity, in that it will cause pain/(re)injury, will form SSBs, thereby prevent the pain patients from disconfirming their beliefs about danger from physical activities. Clinically, this may have important implications to the treatment of chronic pain patients in whom health anxiety is an important factor of their behavior. There is a need to look beyond communicative pain behavior and total avoidance of activities and movements as target for interventions (Tang et al., 2007). The use of behavioral experiments to challenge maladaptive thoughts and beliefs is emphasized by cognitive-behavioral theories of anxiety disorders (e.g. 
Salkovskis et al., 2003). Well, as described behavioral experiments form part of GEXP. It seems therefore interesting to examine whether GEXP has the same results without the addition of behavioral experiments.

In addition to research on the role of safety behavior in exposure, a recent discussion dealt with the distinction between safety behavior and adaptive coping strategies (Thwaites \& Freeston, 2005). Both strategies are aimed at reducing fear but are not intended to avoid catastrophic outcomes. Despite the theoretical distinction of safety behavior and coping, in clinical practice it is often difficult to differentiate between them. Differences can only be determined after evaluating a patient's intention for their use, their perceived function in a specific context, and the impact on catastrophizing. Depending on the feared consequences it seems possible that the same behavior functions both as safety mechanism and a coping strategy. For instance, a component of GEXP, the hierarchy of feared activities, may be perceived by some pain patients as a form of controlling pain and related physiological symptoms, leading them to fear situations in which they fail at control pain and related symptoms. On the other hand, it can also lead to greater perceived control, less fear during GEXP, and might increase patients' motivation at the start of GEXP to expose themselves to feared activities and/or movements. Furthermore, related research suggests that safety behavior promotes adaptive cognitive change (Rachman, 1993).

Based on the above mentioned findings there is a strong need for greater understanding of possible positive and negative consequences of safety behavior and the role of behavioral experiments in fearful pain patients. Is it only the perceived availability of safety behaviors that has a negative effect on fear reduction or is their use necessary? Is moving toward safety behavior during GEXP, rather than away from it, more effective in reducing avoidance behaviors? Do safety behaviors aid to modify catastrophic beliefs because of the fact that pain patients do not misattribute their success to external factors? What is the role of behavioral experiments rather than exposure to challenge catastrophic thoughts and beliefs? Does this allow patients to engage with the process of change behavior? Is there influence of duration to perform a pain-provoking task required to avoid ceiling effects? Most research to safety behavior is done in a laboratory setting by the use of a carefully standardized procedure: how is it in the "real world" (e.g. in patient's home/work situation)? In sum, there still are many questions about the use of safety behaviors of which the answers have important implications for GEXP. 


\section{Random GEXP}

The generalization study in this thesis showed that exposure to multiple as well as 'only' single activities leads to generalization. Despite variability also random exposure is assumed to result in generalization (Schmidt \& Bjork, 1992). In a random condition, patients practiced exposure to feared activities and/or movements in random order, which means that GEXP occurs with disregard of the fear hierarchy. Examining the effects of GEXP based on random GEXP compared to a so-called blocked GEXP in which the pain patient will be exposed to a certain feared activity repeatedly before moving to the next more feared activity might shed new light on this issue.

\section{Spacing of GEXP sessions}

Research has shown that temporally spaced exposure trials often result in a stronger acquisition-type of learning than temporally massed trials (e.g. Scharf et al., 2002). It seems that greater storage strength of memory is gained by partial forgetting between learning episodes, which in turn is presumed to slow the loss of the retrievable memory over time (Bjork \& Bjork, 2003). Currently, the use of GEXP is outlined by a protocol which prescribed two exposure sessions in a week spread over 8 to 9 weeks. Because this schedule is not theoretically driven and GEXP with the protocol as it stands seems to be a successful treatment, both in the short and long run, one could wonder whether an expanding spaced schedule of progressively longer intervals between GEXP sessions or consecutive sessions might have any influence on the outcome of GEXP. In addition, it is important to study this topic in different varieties of pain complaints.

\section{Offsetting context effects}

Despite no return of fear was found in the generalization study as well as in the follow-up period in the other GEXP studies of this thesis and also in the early studies (Vlaeyen et al. 2001, 2002a, 2002b) there are still some questions that have to be answered. Is more return of fear found if re-testing occurred in the original fear acquisition context? For example, the environment of the hospital or proximity of medical facility may be a particularly meaningful context during GEXP. It remains to be determined which contexts play an important role in pain-related fear learning and extinction. Another question to investigate is whether pain-alone exposures also offset context-based renewal effects. 


\section{Neurobiological research}

The majority of neurobiological research on fear learning and exposure has focussed on the amygdala, the prefrontal cortex (PFC) and the hippocampus (see SotresBayon et al., 2006 for a review). The precise function of the amygdala is still unclear. Nevertheless it seems that this area plays a role in fear learning and is involved in extinction learning as well. The hippocampal formation seems to play a critical role in encoding contextual information, which is necessary to form an integrated memory of features of the context before conditioning can take place. Finally, the PFC seems responsible for executive control and decision making, and certain parts of the PFC (i.e., ventral medial) are responsible for emotional regulation, and, in particular, the ability to interpret emotional stimuli and change behavior accordingly (e.g. Craske et al., 2008).

Research to understand the neural mechanisms of GEXP, and emotional regulation during GEXP in particular, is hardly implemented. It seems interesting to understand how the PFC, amygdala and hippocampus interact to fully encode the extinction memory in fearful pain patients and which exact nature role the PFC and amygdala play in fear extinction. Given the role of the PFC, behavioral methods for enhancing PFC throughout GEXP may also prove to be a useful direction for research. If cognitive restructuring enhances the benefits of GEXP alone, it is quite conceivable that this may be done by activation of the PFC. Further, it would be of interest to establish the role of PFC activation in linguistic processing and typical cognitive restructuring and the connection of such activation to the development of new, inhibitory associations and expectancies.

\section{Conclusions}

This dissertation presented some studies to further explore the utility of GEXP. It has been shown that the application of GEXP has considerable promise in the treatment of pain patients who have a clearly articulated pain-related fear. The effects of GEXP generalized from chronic low back pain to other pain problems such as complex regional pain syndrome and posttraumatic neck pain. Significant advances have been made in understanding the behavioral and cognitive mechanisms involved in GEXP and how these mechanisms enhances generalization. Hopefully this work will contribute to better care for patients with chronic pain for who relatively few evidence-based treatment options exist. 

Nederlandse samenvatting (Dutch summary) 
Wanneer traditionele en biomedische behandelingen niet het beoogde resultaat opleveren, worden pijnpatiënten vaak doorverwezen naar revalidatiecentra. De behandelingen binnen deze centra zijn in het algemeen interdisciplinair en gebaseerd op bio-psycho-sociale en gedragsmatige revalidatie modellen. Deze modellen gaan ervan uit dat de ervaren beperkingen niet alleen bepaald worden door de onderliggende pathologie, maar ook door cognitieve factoren (b.v. het denken in catastrofes), gedragsmatige (b.v. het vermijden van bepaalde activiteiten), emotionele (b.v. sombere stemming) en sociale (b.v. algemene opvattingen over ziekte en gezondheid). Methoden die tijdens de behandeling aan bod komen zijn afkomstig uit de beginselen van de operante conditionering (observeerbaar pijngedrag zoals inactiviteit dient de focus van behandeling te zijn) en de cognitieve psychologie (focus ligt op de rol van attributies en verwachtingen, gevoel van controle en probleemoplossingvaardigheden). Onderzoek naar cognitief-gedragsmatige behandelingen laat wisselende resultaten zien. Dientengevolge wordt gesuggereerd om behandelingen nog beter af te stemmen op specifieke kenmerken van de patiënt. In de afgelopen jaren heeft cognitief-gedragsmatig pijnonderzoek zich dan ook gericht op mechanismen die een rol spelen bij de ontwikkeling van een acuut naar een chronisch pijnprobleem. Een aantal studies hebben laten zien dat aan pijn gerelateerde angst een van de sterkste voorspellers is voor de waargenomen variatie in fysieke prestaties bij pijnpatiënten.

Omdat pijngerelateerde angst een substantiële bijdrage levert aan het in stand houden van chronische pijn, is het een belangrijk doelwit binnen de behandeling. Sterker nog, verschillende studies hebben laten zien dat een exposure in vivo behandeling (GEXP) een effectieve behandeling is bij chronische lage rugpijn patiënten die een hoge mate van aan pijn gerelateerde angst rapporteren. Echter, de resultaten van deze studies en de complexiteit van GEXP hebben ook extra vragen opgeroepen. Bijvoorbeeld, welke behandelcomponenten maken GEXP al dan niet succesvol? Wat wordt eigenlijk geleerd tijdens GEXP? Is het uitdoven van pijngerelateerde angst te generaliseren naar andere bewegingen en activiteiten die niet zijn opgenomen in de GEXP? Is GEXP ook toe te passen bij pijnpatiënten met waarneembare weefselpathologie zoals neuropathische pijn? Kortom, antwoorden op deze vragen kunnen bijdragen aan het optimaliseren van GEXP bij pijngerelateerde angst. Het doel van dit proefschrift is om de toepassing van GEXP verder te onderzoeken door na te gaan 1) of GEXP, naast chronische rugpijn, ook positieve effecten oplevert bij andere pijnproblemen, zoals Complex Regionaal Pijn Syndroom en posttraumatische nekpijn, 2) wat de bijdrage is van specifieke behandelcomponenten in het resultaat van GEXP, en 3) of blootstelling aan meerdere stimuli generalisatie verbetert na afloop van GEXP.

In hoofdstuk 1, de inleiding van dit proefschrift, wordt allereerst stilgestaan bij het concept pijn. Pijn is een multidimensionele ervaring, die zowel een sensorische- 
discriminatieve dimensie (b.v. de locatie, duur en intensiteit van de pijn) als affectief-motivationele dimensie heeft (b.v. de onaangenaamheden van pijn en angst voor pijn) bevat, en geen één-op-één relatie heeft met de mate van weefselbeschadiging. Er bestaat grote variabiliteit met betrekking tot de intensiteit van de ervaren pijn tussen individuen. Maar één individu kan pijn varieren afhankelijk van de context waarin het individu zich bevindt. Bovendien, wanneer de pijn aanhoudt en chronisch wordt lijken affectief-motivationele aspecten meer op de voorgrond te treden, terwijl de sensorische een geringere rol spelen bij de pijnervaring. Vervolgens wordt ingegaan op de rol van pijngerelateerde angst als verklaring waarom acute pijnpatiënten een chronisch pijnprobleem met de daarbij horende beperkingen kunnen ontwikkelen. Klassieke of Pavloviaanse conditionering wordt van oudsher beschouwd als model voor de acquisitie van angst. Dit betekent dat een van oorsprong neutrale stimulus wordt gekoppeld met een biologisch relevante en bedreigende stimulus of situatie. Helaas is er weinig onderzoek gedaan naar de rol van klassieke conditionering in de ontwikkeling van pijn-gerelateerde angst. Het is echter waarschijnlijk dat een acute episode van ernstige pijn of andere lichamelijke sensaties wordt gezien als een teken van schade (ongeconditioneerde stimulus: US) en een onschuldige activiteit of beweging (geconditioneerde stimulus: CS) hieraan wordt gerelateerd. Het resultaat van dit leerproces is dat propositionele kennis over de relatie tussen de twee stimuli (de beweging met de toegenomen pijn als teken voor schade) wordt opgeslagen in het geheugen. Wanneer het subject wordt blootgesteld aan dezelfde soort van beweging, zal deze beweging de associatie met de toename van pijn activeren, wat resulteert in het uitlokken van een geconditioneerde respons (CR). Zelfs meer dan het oproepen van een herinnering, zorgt dit leerproces ervoor dat de betreffende beweging resulteert in het activeren van een actieve verwachting, namelijk die van een bedreigende gebeurtenis. Herhaling van het leerproces zorgt er ook voor dat de beweging een goede voorspeller wordt van toenemende pijn als teken van schade: 'Wanneer ik mijn vierjarig kind optil, dan ervaar ik onmiddellijk meer pijn in mijn rug wat duidelijk aangeeft dat mijn wervels versleten zijn' en 'Als ik mijn kind niet optil, dan zal de pijn in mijn rug niet toenemen, hetgeen betekent dat ik mijn wervels niet nog meer ga beschadigen'. Het subject legt niet alleen een verband tussen twee gebeurtenissen (associatief leren), maar leert ook dat de ene gebeurtenis de andere voorspelt (verwachtingsleren). Verwachtingsleren resulteert uiteindelijk in generalisatie van angst voor activiteiten, bewegingen en/of situaties vergelijkbaar met de onschadelijke beweging die de pijn heeft uitgelokt. De nieuwe en bedreigende betekenis van een oorspronkelijk neutrale beweging zorgt ervoor dat het subject zijn gedrag zal veranderen. Meer en meer activiteiten, bewegingen en/of activiteiten zullen worden vermeden. Pijngerelateerde angst kan zich ontwikkelen op minstens drie verschillende manieren: directe ervaringen (bijvoorbeeld een specifieke beweging verhoogt de pijn of de zwelling van de gebruikte arm); verbale informatie (bijvoorbeeld een arts die aan- 
geeft dat er spraken is van artrose); of het observeren van anderen (bijvoorbeeld een vriend zittend in een rolstoel vanwege dezelfde pijnklachten of niet in staat is om te werken). Naast vermijding en ontsnappingsgedrag en verhoogde pijnervaring is pijngerelateerde angst gerelateerd aan denken in catastrofes over pijn, hypervigilantie, functionele beperkingen, veranderingen op centraal niveau, motorische abnormaliteiten, en depressiviteit. Exposure lijkt een geschikte behandeling. Het wordt gezien als een proces waarbij de patiënt tijdens een bepaalde periode herhaaldelijk wordt blootgesteld aan bedreigende situaties terwijl aversieve consequenties uitblijven. Dit proces wordt ook wel extinctie genoemd. Extinctie verwijst naar een zich herhalende presentatie van een geconditioneerde stimulus (CS: bijvoorbeeld een onschuldige activiteit) in de afwezigheid van de ongeconditioneerde stimulus (US: bijvoorbeeld een pijnproducerende of angstopwekkende stimulus) waarmee de CS voorheen was gekoppeld. Onderzoek heeft laten zien dat extinctie niet leidt tot het uitdoven van de angst en dat het veranderen van de context waarin extinctie heeft plaatsgevonden en het aanbieden van een onverwachte US ervaring na afloop van extinctie kan leiden tot een gedeeltelijk herstel van de ogenschijnlijk gedoofde angstreacties. Extinctie in wisselende contexten en herhaling lijkt in dit opzicht belangrijk te zijn. Bovendien blijkt dat cognitieve processen die disfunctionele overtuigingen beïnvloeden onderdeel uitmaken van de exposure therapie.

Voor pijnpatiënten die een hoge mate van pijngerelateerde vrees rapporteren is een 'graded exposure in vivo' (GEXP) ontwikkeld om extinctie van aan pijn gerelateerde angst te bewerkstelligen. Tijdens GEXP wordt de patiënt tijdens een bepaalde periode herhaaldelijk wordt blootgesteld aan bedreigende situaties. GEXP is zeer gestructureerd, geprotocolleerd, op maat gesneden, en streeft naar een normaal patroon van het dagelijks functioneren, met inbegrip van volledige terugkeer naar het werk. Zowel experimentele single-case studies als gerandomiseerde gecontroleerde studies (RCT) hebben aangetoond dat GEXP effectief is in het verminderen van functionele beperkingen bij patiënten met chronische lage rugpijn. Echter, bij twee RCT's was deze vermindering niet statistisch significant ten opzichte van een controle groep die op een wachtlijst stonden, gebruikelijke therapie (contact met een huisarts, medicatie, fysiotherapie) aangeboden kregen, of deelnamen aan een programma waarin stapsgewijs activiteiten werden opgebouwd. Over het geheel genomen is een van de meest opvallende kenmerken in deze studies het genoemde belang van de educatieve component van de exposure behandeling. Tijdens de educatie wordt het vrees-vermijdings-model geillustreerd en uitgelegd. Ook wordt beschreven dat het belangrijk is om tijdens de exposure verschillende angstopwekkende activiteiten aan te bieden en dat therapeuten over de benodigde competenties beschikken. Tot slot, de resultaten van de single-case studies, alsook die van de RCT's, zijn hoopvol met betrekking tot generalisatie van extinctie. 
Hoofdstuk 2 van dit proefschrift is gericht op de rol van pijngerelateerde angst bij patiënten met een Complex Regionaal Pijn Syndroom type I (CRPS-I), ook wel posttraumatische dystrofie genoemd. Twee studies worden gepresenteerd. Studie I omvat een steekproef van patiënten met een (sub)acute CRPS-I die zijn verwezen naar een poliklinische pijnpoli. In Studie II zijn patiënten met chronische CRPS-I en die lid zijn van de Nederlandse patiëntenvereniging uitgenodigd om deel te nemen aan een onderzoek. De resultaten van Studie I laten zien dat de hevigheid van de pijn, maar niet pijngerelateerde angst zoals gemeten met de Tampa Schaal voor Kinesiofobie (TSK), gerelateerd is aan de mate van functionele beperkingen bij een (sub)acute CRPS-I. Deze resultaten zijn niet consistent met eerder onderzoek bij patiënten met pijn aan het bewegingsapparaat waarbij een hoge TSK-score wel gerelateerd was aan de mate van functionele beperkingen, en zelfs meer nog dan de pijn zelf. Hoewel er een gebrek is aan consensus tussen onderzoekers, wordt CRPS-I gezien als een neuropathisch pijnsyndroom. Daarom is verondersteld dat de TSK niet gevoelig genoeg is om de mate van pijngerelateerde angst te meten, en is in Studie II ook een instrument gebruikt die bestaat uit foto's van dagelijkse activiteiten (PHODA) om pijngerelateerde angst te meten. De resultaten van Studie II laten zien dat bij patiënten met een chronische CRPS-I, naast de hevigheid van de pijn, ook de aan pijn gerelateerde angst, gemeten met de PHODA, een significante voorspeller is voor de mate van functionele beperkingen. Hoewel replicatie studies noodzakelijk zijn, lijkt het gerechtvaardigd om te concluderen dat de rol van pijngerelateerde angst een veelbelovende onderzoeksrichting is naar het begrijpen van de ervaren functionele beperkingen bij CRPS-I.

Hoofdstuk 3 en 4 beschrijven een tweetal studies waarin door middel van singlecase experimenten met herhaalde metingen de effectiviteit van GEXP is onderzocht bij patiënten met posttraumatische nekpijn (PTNP) en CRPS-I. Patiënten die deelnamen aan de studie rapporteerden een hoge mate van pijngerelateerde angst. In de PTNP studie hebben patiënten zowel een GEXP alsook een operant 'graded actvity' (OPE) programma doorlopen. Het doel van OPE is gezond gedrag bevorderen en pijngedrag te verminderen. Er wordt vanuit gegaan dat inactiviteit (pijngedrag) leidt tot een verminderde belastbaarheid, welke pijn bevorderd, en dat het verhogen van het activiteitenniveau en de spiersterkte in de toekomst waarschijnlijk zal leiden tot minder beperkingen. De volgorde waarin GEXP en OPE werd aangeboden gebeurde op basis van toeval. Door gebruik te maken van dagboekjes hebben beide studies laten zien dat tijdens GEXP er niet alleen een significatie vermindering plaatsvindt van pijngerelateerde angst en het denken in catastrofes over pijn, maar ook voor wat betreft pijnintensiteit. Tevens rapporteren alle patiënten tijdens GEXP doelen te kunnen bereiken die betrekking hebben op de uitvoering van activiteiten die bij aanvang van de studies als onmogelijk werden verondersteld. Gestandaardiseerde vragenlijsten over pijngerelateerde angst, pijnintensiteit en functionele beperkin- 
gen, die voorafgaande en na afloop van iedere interventie werden afgenomen laten alleen na afloop van GEXP een significante afname zien. Om het niveau van dagelijkse fysieke activiteit te kwantificeren werd door PTNP patiënten tijdens deze perioden ook een ambulante bewegingsmeter gedragen. Een significante toename van de dagelijkse fysieke activiteit was wederom alleen te zien na afloop van GEXP. Tenslotte, het meest opvallende resultaat was dat fysiologische verschijnselen en symptomen, zoals abnormale gevoeligheid voor prikkeling van de zintuigen, oedeem, veranderde lichaamskleur en temperatuur, en een verstoorde zweetsecretie, die verband houden met CRPS positief werden beïnvloed door GEXP. Tijdens een follow-up meting van 6 maanden bleken alle hierboven beschreven positieve resultaten, als gevolg van GEXP, nog steeds aanwezig te zijn. Beide studies ondersteunen de effectiviteit van GEXP bij patiënten met PTNP en CRPS-I die een hoge mate van pijngerelateerde angst rapporteren.

Klinisch onderzoek, dat mede heeft geleid tot de vraagstellingen binnen dit proefschrift, heeft laten zien dat bij chronische lage rugpijn patiënten vrij snel na de start van het GEXP programma een abrupte afname van pijngerelateerde angsten en cognities plaatsvindt. Deze abrupte veranderingen zijn meer een kenmerk van inzichtelijk leren in plaats van de gebruikelijke leermethode van vallen en opstaan. Als het GEXP protocol is gevolgd, zou het dus zo kunnen zijn dat het educatieve deel aan het begin van GEXP heeft bijgedragen tot dit inzicht. Hoofdstuk $\mathbf{5}$ van dit proefschrift beschrijft een studie waarin de bijdrage van de educatie aan het verminderen van pijngerelateerde angst, en de daaraan verbonden beperkingen in fysiek functioneren, bij chronische lage rugpijn patiënten is onderzocht. Na een periode waarin geen enkele behandeling is gevolgd, kregen alle patiënten het educatieve deel van de GEXP aangeboden, gevolgd door wederom een behandelvrije periode. Patiënten werden vervolgens willekeurig toegewezen aan hetzij GEXP of een gedragsmatige oefentherapie (OPE). Ook nu weer werd een dagboekje gebruikt om dagelijkse veranderingen van pijnintensiteit, pijngerelateerde angst, het denken in catastrofes over pijn en de moeilijkheid om een bepaalde activiteit uit te voeren te meten. Tevens werd voorafgaand en na afloop van iedere interventie en tijdens een 6maanden follow-up gestandaardiseerde vragenlijsten afgenomen om pijngerelateerde angst, gevoeligheid voor pijn, pijnintensiteit en ervaren beperkingen als gevolg van de pijn te meten, en droegen de patiënten gedurende één week een ambulante bewegingsmeter. De resultaten van de dagelijkse metingen laten zien dat pijngerelateerde angst inderdaad positief werd beïnvloed door de educatie. Echter, het uitvoeren van relevante dagelijkse activiteiten werd niet beïnvloed door de educatie, maar verbeterde alleen onder invloed van GEXP. Ook tijdens de 6maanden follow-up werden deze verbeteringen alleen gevonden bij patiënten die een GEXP hadden gevolgd, en rapporteerde deze groep zelfs een significante afname in pijnintensiteit. 
Ondanks het succes van GEXP bij chronische pijn is het nog de vraag of extinctie generaliseert naar nieuwe bedreigende activiteiten. Uit dierexperimenteel onderzoek naar de terugkeer van angst is gebleken dat extinctie niet betekent afleren betekent. Het lijkt erop dat de gedoofde angstreactie na een tijdje weer spontaan kan ontstaan, of opnieuw kan optreden bij confrontatie met een nieuwe CS-US gebeurtenis of een onverwachte US. Tevens lijkt extinctie ook context gevoelig. Exposure onderzoek bij lage rugpijn patiënten heeft laten zien dat wat is geleerd tijdens extinctie niet noodzakelijkerwijs generaliseert naar andere situaties, zelfs niet wanneer extinctie plaatsvindt in een situatie waarin de oorspronkelijke angst is ontstaan. Hoofdstuk 6 van dit proefschrift beschrijft een studie met als primaire doel een eerdere studie te repliceren waarin gevonden werd dat exposure aan meerdere stimuli en/of contexten generalisatie bevordert. Door gebruik te maken van een single-case experimenteel design werd onderzocht of de effecten van GEXP bij angstige CRPS-I patiënten generaliseren naar bedreigende activiteiten waaraan de patiënten tijdens GEXP niet zijn blootgesteld. Twee GEXP condities bestaande uit 15 sessies van één uur werden met elkaar vergeleken. Conditie I bestond uit een GEXP programma van minimaal 15 activiteiten waaraan patiënten slechts één keer werden blootgesteld en Conditie II uit 3 activiteiten waaraan patiënten vijf maal werden blootgesteld. In beide condities werd generalisatie van extinctie getest door patiënten na een succesvolle GEXP bloot te stellen aan een nieuwe activiteit. De veronderstelling was dat generalisatie van extinctie wordt bevorderd door de conditie waarin de meerdere activiteiten werden aangeboden. In tegenstelling tot wat werd verwacht presteerden de patiënten uit beide condities even goed tijdens de test exposure. Bij alle patiënten was sprake van generalisatie naar een nieuwe activiteit direct na afloop van GEXP en tijdens een 6-maanden follow-up. Over het geheel genomen lijkt het erop dat, ongeacht de aard van GEXP, extinctie generaliseert naar nieuwe bedreigende activiteiten.

Tenslotte worden in hoofdstuk $\mathbf{7}$ allereerst de bevindingen van dit proefschrift samengevat en vervolgens ter discussie gesteld. De studies in dit proefschrift hebben aangetoond dat GEXP een veelbelovende behandeling is voor chronische pijnpatiënten die een hoge mate van pijngerelateerde angst rapporteren. De effecten van GEXP generaliseren van chronische lage rugpijn naar andere pijnproblemen zoals CRPS-I en PTNP. Aanzienlijke vooruitgang is geboekt in het begrijpen van de gedragsmatige en cognitieve mechanismen die betrokken zijn bij GEXP en hoe deze mechanismen generalisatie van extinctie versterken. Desondanks blijven er nog steeds onduidelijkheden bestaan over de cognitieve processen die ten grondslag liggen aan het succes van GEXP. Er is daarom behoefte aan meer theoretisch gericht onderzoek. Studies gericht op verwachtingen (bijvoorbeeld, voorgenomen kennis over het verband tussen een specifieke activiteit en de daaropvolgende pijn als 
potentieel teken van schade), het wel of niet toepassen van veiligheidsgedrag tijdens GEXP, willekeurige exposure (GEXP wordt aangeboden zonder acht te slaan op de angsthiërarchie), het leereffect van tijdelijk gespreide en opeenvolgende exposure sessies, het neutraliseren van de context (bijvoorbeeld, het gegeven dat GEXP plaatsvindt in het ziekenhuis kan een belangrijke rol spelen), en neuro-cognitieve processen werpen misschien meer licht op de mechanismen die ten grondslag liggen aan de associaties tussen pijngerelateerde angst en chronische pijn. 


\section{References}


Aaronson NK, Muller M, Cohen PDA, Essink ML, Fekkes M, Sanderman R, Sprangers MA, te Velde A, Verrips E. Translation, validation and norming of the Dutch language version of the SF-36 Health Survey in community and chronic disease populations. J Clin Epidemiol 1998; 51: 1171-8. (chapter 2)

Abramson JH, Gahlinger PM. Computer programs for epidemiologists. 3rd ed. London: Brixton Books; 1999. (chapter 2)

Ajzen I. Nature and operation of attitudes. Annual Rev of Psychol 2001; 52: 27-58. (chapter 5)

Anderson KB, Anderson CA, Dill KE, and Deuser WE. The interactive relations between trait hostility, pain, and aggressive thoughts. Aggressive Behavior 1998; 24: 161-171. (chapter 3)

Andersen RA, Buneo CA. Sensorimotor integration in posterior parietal cortex. Advan Neurol 2003; 93 : 159-177. (chapter 7)

Apkarian AV, Thomas PS, Krauss BR, Szeverenyi NM. Neurosci Lett 2001; 311: 193-197. (chapter 1)

Apkarian AV, Bushnell MC, Treede RD, Zubieta JK. Human brain mechanisms of pain perception and regulation in health and disease. Eur J Pain 2005; 9: 463-484. (chapter 1)

Arntz A, Dreessen L, Merckelbach H. Attention, not anxiety, influences pain. Behav Res Ther 1991; 29: 41-50. (chapter 4)

Arntz A, de Jong P. Anxiety, attention and pain. J Psychosom Res 1993; 37: 423-432. (chapter 4)

Arntz A, Dreessen L, de Jong $P$. The influence of anxiety on pain: attentional and attributional mediators. Pain 1994; 56: 307-314. (chapter 1, 4)

Arntz A, Claassens L. The meaning of pain influences its experienced intensity. Pain 2004; 109: $20-25$. (chapter 1, 3, 4)

Arrindell WA, Ettema JHM. Symptom checklist (SCL-90): Handleiding bij een multidimensionele psychopathologie-indicator. Lisse: Swets; 1986. (chapter 3, 6)

Asmundson GJ, Kuperos JL, Norton GR. Do patients with chronic pain selectively attend to pain-related information?: Preliminary evidence for the mediating role of fear. Pain 1997; 72: 27-32. (chapter 3)

Asmundson GJ, Norton PJ, Norton GR. Beyond pain: The role of fear and avoidance in chronicity. Clin Psychol Rev 1999; 19: 97-119. (chapter 1, 3, 6)

Asmundson GJ, Nicholas Carleton R. Fear of pain is evaluated in adults with co-occuring trauma-related stress and social anxiety symptoms. Cogn Behav Ther 2005; 34: 248-255. (chapter 7)

Baeyens F, Vansteenwegen D, Beckers T, Hermans D, Kerkhof I, De Ceulaer A. Extinction and renewal of Pavlovian modulation in human sequential Feature Positive discrimination learning. Learn Mem 2005; 12: 178-192. (chapter 6)

Bantick SJ, Wise RG, Ploghaus A, Clare S, Smith SM, Tracey I. Imaging how attention modulates pain in humans using functional MRI. Brain 2002; 125: 310-319. (chapter 1)

Barlow DH. Anxiety and Its Disorders: The Nature and Treatment of Anxiety and Panic. New York, NY: The Guilford Press; 2002. (chapter 1, 3)

Barlow DH. Psychological treatments. Am Psychol 2004; 59: 869-78. (chapter 1)

Barnsley L, Lord S, Bogduk N. Whiplash injury. Pain 1994; 58: 283-307. (chapter 3)

Beck AT, Emery G. Anxiety disorders and Phobias: a cognitive perspective. New York: Basic Books, Inc.; 1985. (chapter 1)

Bergstrand R, Vedin A, Wilhelmsson C, Wilhelmsen L. Bias due to non-participation and heterogenous sub-groups in population surveys. J Chronic Dis 1983; 36: 725-728. (chapter 2)

Berkowitz L. Pain and aggression: Some findings and implications. Motivation Emotion 1993; 17: 277-293. (chapter 7)

Berkowitz L, Harmon-Jones E. Toward an understanding of the determinants of anger. Emotion 2004; 4: 107-130. (chapter 3, 7)

Bjork EL, Bjork RA. Intentional forgetting can increase, not decrease, residual influences of to-beforgotten information. J Exp Psychol Learn Mem Cogn 2003; 29: 524-531. (chapter 7)

Bland JM, Altman DG. Statistical methods for assessing agreement between two methods of clinical measurement. Lancet 1986; 8: 307-310. (chapter 4, 6)

Blyth FM, March LM, Nicholas MK, Cousins MJ. Chronic pain, work performance and litigation. Pain 2003; 103: 41-47.(chapter 7) 
Boersma K, Linton SJ, Overmeer T, Jansson M, Vlaeyen J, de Jong J. Lowering fear-avoidance and enhancing function through exposure in vivo: A multiple baseline study across six patients with back pain. Pain 2004; 108: 8-16. (chapter 1, 3, 4, 6)

Bohner G, Wänke M. Attitudes and Attitude Change. East Sussex, Uk: Psychology Press, 2002. (chapter 5)

Borkovec TD, Nau SD. Credibility of analogue therapy rationales. J Behav Ther Exper Psychol 1972; 3: 257-260. (chapter 4, 6)

Bouton ME, King DA. Contextual control of the extinction of conditioned fear: tests for the associative value of the context. J Exp Psychol Anim Behav Process 1983; 9: 248-265. (chapter 6)

Bouton ME, Swartzentruber D. Sources of relapse after extinction in Pavlovian and instrumental learning. Clin Psychol Review 1991; 11: 123-140. (chapter 1, 6)

Bouton ME. Context, time, and memory retrieval in the interference paradigms of Pavlovian learning. Psychol Bull1 1993; 114: 80-99. (chapter 1)

Bouton ME, Ricker ST.Renewal of extinquished responding in a second context. Anim Learn Behav 1994; 22: 317-324. (chapter 1)

Bouton ME, Mineka S, Barlow DH. A modern learning theory perspective on the etiology of panic disorder. Psychol Rev1 2001; 108: 4-32. (chapter 1)

Bouton ME. Context, ambiguity, and unlearning: Sources of relapse after behavioral extinction. Biol Psychiatry 2002; 52: 976-986. (chapter 1, 3)

Bouton ME. Context and behavioral processes in extinction. Learn Mem1 2004; 11: 485-494. (chapter 1)

Bruehl S, Harden RN, Galer BS, Saltz S, Bertram M, Backonja M, Gayles R, Rudin N, Bhugra MK, StantonHicks M. External validation of IASP diagnostic criteria for complex regional pain syndrome and proposed research diagnostic criteria. International association for the study of pain. Pain 1999; 81 : 147-154. (chapter 2, 4)

Bruehl SP. Do psychological factors play a role in the onset and maintenance of CRPS? In: Harden RN, Baron R, Jänig W (Eds.). Complex Regional Pain Syndrome, Progress in Pain Research and Management, Vol. 22. Seattle: IASP Press; 2001. (chapter 2)

Bruehl S, Chung OY, Burns JW. Differential effects of expressive anger regulation on chronic pain intensity in CRPS and non-CRPS limb pain patients. Pain 2003; 104: 647-654. (chapter 7)

Bruehl SP. Psychological interventions. In Wilson PR, Stanton-Hicks M, Harden RN (Eds.). CRPS: Current Diagnosis and Therapy. Seattle: IASP Press 2005; 201-216. (chapter 2)

Bruehl S, Burns JW, Chung OY, Chont M. Pain-related effects of trait anger expression: neural substrates and the role of endogenous opioid mechanisms. Neurosci Biobehav Rev 2009; 33(3): 475-491. (chapter 1 )

Buer N, Linton SJ. Fear-avoidance beliefs and catastrophizing: Occurrence and risk factor in back pain and ADL in the general population. Pain 2002; 99: 485-491. (chapter 3)

Burns JW, Glenn B, Bruehl S, Harden RN, Lofland K. Cognitive factors influence outcome following multidisciplinary chronic pain treatment: a replication and extension of a cross-lagged panel analysis. Behav Res Ther1 2003; 41: 1163-1182. (chapter 1)

Burns JW, Bruehl S. Anger management style, opioid analgesic use, and chronic pain severity: a test of the opioid-deficit hypothesis. J Behav Med 2005; 28: 555-563. (chapter 7)

Bussman JBJ, Laar van de YM, Neleman MP, Stam HJ. Ambulatory accelerometer to quantify motor behaviour in patients after failed back surgery: avalidation study. Pain 1998; 74: 153-161. (chapter 5)

Bussmann JB, Martens WL, Tulen JHM, Schasfoort FC, vd Berg-Emons HJG, Stam HJ. Measuring daily behaviour using ambulatory accelerometry: the activity monitor. Behav Res Methods Instrum Comp 2001; 33: 349-356. (chapter 4)

Butler SH. Disuse and CRPS. In: Harden RN, Baron R, Jänig W (Eds). Complex Regional Pain Syndrome, Progress in Pain Research and Management vol. 22. Seattle; IASP Press 2001: 141-150. (chapter 4)

Butler AC, Chapman JE, Forman EM, Beck AT. The empirical status of cognitive-behavioral therapy: A review of meta-analyses. Clin Psychol 2006; 26: 17-31. (chapter 3)

Cannon WB. Bodily changes in pain, hunger, fear and rage: An account of recent researchers into the functions of emotional excitement, $2^{\text {nd }}$ ed. New York, NY: Guilford Press, 1929. 
Carleton RN, Asmundson GJ, Collimore KC, Ellwanger J. Strategic and automatic threat processing in chronic musculoskeletal pain: a startle probe investigation. Cogn Behav Ther 2006; 35: 236-247. (chapter 7)

Chelonis JJ, Calton JL, Hart JA, Schachtman TR. Attenuation of the renewal effect by extinction in multiple contexts. Learn Motivat 1999; 30: 1-14. (chapter 7)

Clark DM. A cognitive approach to panic. Behav Res Ther 1986; 24: 461-470. (chapter 3)

Collins BN, Brandon TH. Effects of Extinction Context and Retrieval Cues on Alcohol Cue Reactivity Among Nonalcoholic Drinkers. J Consult Clin Psychol 2002; 70: 390-397. (chapter 7)

Cook AJ, Brawer PA, Vowles KE. The fear-avoidance model of chronic pain: validation and age analyses using structural equation modelling. Pain1 2006; 121: 195-206. (chapter 1)

Cox BJ, Endler NS, Lee PS, Swinson RP. A meta-analysis of treatments for panic disorder with agoraphobia: imipramine, alprazolam, and in vivo exposure. J Behav Ther Exp Psych 1992, 23, 175-182. (chapter 6)

Craig AD. How do you feel? Interoception: the sense of the physiological condition of the body. Nat Rev Neurosci1 2002; 3: 655-666. (chapter 1)

Craske MG, Kircanski K, Zelikowsky M, Mystkowski J, Chowdhury N, Baker A. Optimizing inhibitory learning during exposure therapy. Behav Res Ther 2008; 46: 5-27. (chapter 1, 6)

Crawford JR, Khan RJ, Varley GW. Early management and outcome following soft tissue injuries of the neck: A randomised controlled trial. Injury 2004; 35: 891-895. (chapter 3)

Créac'h C, Henry P, Caillé JM, Allard M. Functional MR imaging analysis of pain-related brain activation after acute mechanical stimulation. AJNR Am J Neuroradiol1 2000; 21: 1402-1406. (chapter 1)

Crombez G, Vervaet L, Baeyens F, Lysens R, Eelen P. Do pain expectancies cause pain in chronic low back pain patients? A clinical investigation. Behav Res Ther 1996 ; 34 : 919-925. (chapter 1, 4, 6)

Crombez G, Vervaet L, Lysens R, Baeyens F, Eelen P. Avoidance and confrontation of painful, backstraining movements in chronic back pain patients. Behav Mod 1998; 22: 62-77. (chapter 6)

Crombez G, Vlaeyen JWS, Heuts PH, Lysens R. Pain-related fear is more disabling than pain itself: evidence on the role of pain-related fear in chronic back pain disability. Pain 1999; 80: 329-339. (chapter $1,2,4,6)$

Crombez G, Eccleston C, Vlaeyen JWS, Vansteenwegen D, Lysens R, Eelen P. Exposure to Physical Movements in Low Back Pain Patients: Restricted Effects of Generalization. Health Psychol 2002; 21: 573578. (chapter 1, 6)

Crombez G, Eccleston C, Van den Broeck A, Goubert L, Van Houdenhove B. Hypervigilance to pain in fibromyalgia: the mediating role of pain intensity and catastrophic thinking about pain. Clin J Pain1 2004; 20(2): 98-102. (chapter 1)

Crombez G, Van Damme S, Eccleston C. Hypervigilance to pain: an experimental and clinical analysis. Pain1 2005; 116: 4-7. (chapter 1, 5)

Cup E, van de Ven-Stevens L, Corstens-Mignot M. Radboud Skills Test (RST): A specific disease-related functional hand-test for patients with Complex Regional Pain Syndrom type I of one upper extremity (translated from Dutch). Nederlands Tijdschrift voor Ergotherapie 1999; 27: 152-156. (chapter 6)

Cup E, Scholte op Reimer W, Thijssen M, van Kuyk-Minis M. Reliability and Validity of the Canadian Occupational Performance Measure in Stroke Patients. Clin Rehab 2004; 17: 402-409. (chapter 6)

Daniel HC, Narewska J, Serpell M, Hoggart B, Johnson R, Rice AS. Comparison of psychological and physical function in neuropathic pain and nociceptive pain: Implications for cognitive behavioral pain management programs. Eur J Pain 2008; 12: 731-741. (chapter 2)

Davey GCL. Classical conditioning and the acquisition of human fears and phobias: a review and synthesis of the literature. Adv Behav Res Ther 1992; 14: 29-66. (chapter 6)

Davis M, Ressler K, Rothaum BO, Richardson R. Effects of D-cycloserine on extinction: Translation from preclinical and clinical work. Bio Psychiatry 2006; 60: 369-375. (chapter 1)

De Boer IG, van Drie-Verschoor P, Huijmans AM, Corstens-Mignot MAAMG, Cup EHC, Berendsen EMJ. Intra- and Interrater Reliability of the Radboud Skills Test (translated from Dutch). Nederlands Tijdschrift voor Ergotherapie 2001; 29: 15-19. 
Dedding C, Cardol M, Eyssen IC, Dekker J, Beelen A. Validity of the Canadian Occupational Performance Measure: a client-centred outcome measurement. Clinical Rehabilitation 2004; 18: 660-667.

DeGood DE, Kiernan B. Perception of fault in patients with chronic pain. Pain 1996; 64: 153-159. (chapter 7)

De Houwer J, Crombez G, Baeyens F. Avoidance behavior can function as a negative occasion setter. J Exp Psychol Anim Behav Process1 2005; 31: 101-106. (chapter 1)

De Jong PJ. Implicit self-esteem and social anxiety: differential self-favouring effects in high and low anxious individuals. Behav Res and Ther 2002; 40: 501-508. (chapter 5)

De Jong PJ, van den Hout MA, Rietbroek H, Huijding J. Dissociations between implicit and explicit attitudes toward phobic stimuli. Cognition and Emotion 2003; 17: 521-545. (chapter 5)

De Jong JR, Vlaeyen JWS, Onghena P, Cuypers C, Den Hollander M, Ruijgrok J. Reduction of pain-related fear in complex regional pain syndrome type I: The application of graded exposure in vivo. Pain 2005a; 116: 264-275. (chapter 2, 6)

De Jong JR, Vlaeyen JWS, Onghena P, Goossens MEJB, Geilen M, Mulder H. Fear of movement/(re)injury in chronic low back pain: Education or exposure in vivo as mediator to fear reduction? Clin J Pain 2005b; 21: 9-17. (chapter 3, 4)

De Wied M, Verbaten MN. Affective pictures processing, attention, and pain tolerance. Pain 2001; 90 : 163-172. (chapter 2)

Deyo RA, Battie M, Beurskens AJ, Bombardier C, Croft P, Koes B, Malmivaara A, Roland M, Von Korff M, Waddell G. Outcome measures for low back pain research: a proposal for standardized use. Spine 1998; 23: 2003-13. (chapter 5)

Dirikx T, Hermans D, Vansteenwegen D, Baeyens F, Eelen P. Reinstatement of extinguished conditioned responses and negative stimulus valence as a pathway to return of fear in humans. Learn Mem 2004; 11: 549-554. (chapter 1)

Dubbers AT, Vikström MH, De Jong JR. The Photograph series of Daily Activities (PHODA): Cervical Spine and Shoulder. CD-rom version 1.2: Hogeschool Zuyd, University Maastricht and Institute for Rehabilitation Research (iRv). The Netherlands, 2003. (chapter 1, 2, 3, 4, 6)

Edgington ES. Randomization tests for one-subject operant experiments. J Psychol 1975; 90: 57-68. (chapter 3, 6)

Edgington ES. Overcoming obstacles to single-subject experimentation. J Educ Stat 1980; 5: 261-267. (chapter 3, 6)

Edgington ES. Nonparametric tests for single-case experiments. In: T.R. Kratochwill and J.R. Levin (Eds.). Single-Case Research Design and Analysis: New Directions for Psychology and Education. Hillsdale, NJ; Lawrence Erlbaum Associates 1992: 133-157. (chapter 3, 4)

Edgington ES. Randomization Tests (3rd ed.). New York; Marcel Dekker 1995. (chapter 4, 5)

Edgington ES, Onghena P. Randomization Tests (4th ed.). Boca Raton, FL; Chapman and Hall/CRC 2007. (chapter 3, 6)

Edwards B, Clarke V. The psychological impact of a cancer diagnosis on families: the influence of family functioning and patients' illness characteristics on depression and anxiety. Psychooncology1 2004; 13: 562-576. (chapter 7)

Edwards RR, Bingham CO 3rd, Bathon J, Haythornthwaite JA. Catastrophizing and pain in arthritis, fibromyalgia, and other rheumatic diseases. Arthritis Rheum 2006; 55: 325-332. (chapter 7)

Egle UT, Hoffmann SO. Psychosomatic correlations of sympathetic reflex dystrophy (Südecks disease). Review of the literature and initial clinical results. Psychother Psychosom Med Psychol 1990; 40: 123-135. (chapter 4)

Eisenberg E, Melamed E. Can Complex Regional Pain Syndrome be painless? Pain 2003; 106: 263-267. (chapter 7)

Evers AWM, Kraaimaat FW, Geene R, Jacobs JWG, Bijlsma JWJ. Pain coping and social support as predictors of long-term functional disability and pain in early rheumatoid arthritis. Behav Res Ther 2003; 41: 1295-1310. (chapter 7) 
Farchione TJ. Effects of overlearning on return of fear. Dissertation Abstracts International: Section B. Scienc Engin 2002; 62: 5371. (chapter 6)

Fazio RH. How do attitudes guide behavior? In MR Sorrentino, ET Higgings (Eds.). The handbook of motivation and cognition: Foundations of Social Behavior. New York; Guildford Press 1986: 204-43. (chapter 5)

Fazio $\mathrm{RH}$. A practical guide to the use of response latency in social psychological research. In: Hendrick C, Clark MS (Eds.). Research methods in personality and social psychology. Thousand Oaks, CA: Sage Publications, Inc, 1990: 74-97. (chapter 7)

Fazio $\mathrm{RH}$, Jackson JR, Dunton BC, Williams CJ. Variability in automatic activation as an unobstrusive measure of racial attitudes: a bona fide pipeline? J of Pers and Soc Psychol 1995; 69: 1013-1027. (chapter 5)

Feleus A, van Dalen T, Bierma-Zeinstra SM, Bernsen RM, Verhaar JA, Koes BW, Miedema HS. Kinesiophobia in patients with non-traumatic arm, neck and shoulder complaints: a prospective cohort study in general practice. BMC Musculoskelet Disord1 2007; 8: 117. (chapter 1)

Ferrari R, Russell AS, Carroll L, Cassidy JD. A re-examination of the whiplash associated disorders (WAD) as a systemic illness. Ann Rheum Dis 2005; 64: 1337-1342. (chapter 3)

Ferron J, Onghena P. The power of randomization tests for single-case phase designs. J Exp Educ 1996; 64: 231-239. (chapter 3)

Flink IK, Nicholas MK, Boersma K, Linton SJ. Reducing the threat value of chronic pain: A preliminary replicated single-case study of interoceptive exposure versus distraction in six individuals with chronic back pain. Behav Res Ther 2009; 47: 721-728. (chapter 7)

Flor H, Turk DC, Birbaumer N. Assessment of stress-related psychophysiological reactions in chronic back pain patients. J Consult Clin Psychol 1985; 53: 354-364. (chapter 3)

Flor H, Denke C, Schaefer M, Grusser S. Effect of sensory discrimination training on cortical reorganisation and phantom limb pain. Lancet 2001; 357: 1763-1764. (chapter 4)

Flor $\mathrm{H}$. The modification of cortical reorganization and chronic pain by sensory feedback. Appl Psychophysiol Biofeedback1 2002; 27: 215-27. (chapter 1)

Förderreuther S, Sailer U, Straube A. Impaired self-perception of the hand in complex regional pain syndrome (CRPS). Pain1 2004; 110: 756-761. (chapter 7)

Fordyce WE. Behavioral methods for chronic pain and illness. St. Louis, MO; Mosby 1976. (chapter 3, 5)

Foster NE, Pincus T, Underwood M, Vogel S, Breen A, Harding G. Treatment and the process of care in musculoskeletal conditions: A multidisciplinary perspective and integration. Orthop Clin North Am 2003; 34: 239-244. (chapter 3)

Frettlöh J, Hüppe M, Maier C. Severity and specificity of neglect-like symptoms in patients with complex regional pain syndrome (CRPS) compared to chronic limb pain of other origins. Pain 2006; 124: 184189. (chapter 7)

Fritz JM, George SZ, Delitto A. The role of fear-avoidance beliefs in acute low back pain: Relationships with current and future disability and work status. Pain 2001; 94: 7-15. (chapter 3)

Fritz JM, George SZ. Identifying psychosocial variables in patients with acute work-related low back pain: the importance of fear-avoidance beliefs. Phys Ther 2002; 82: 973-983. (chapter 2)

Galer BS, Jensen MP. Development and preliminary validation of a pain measure specific to neuropathic pain: The Neuropathic Pain Scale. Neurology 1997; 48: 332-338. (chapter 2)

Galer BS, Jensen M. Neglect-like symptoms in complex regional pain syndrome: results of a selfadministered survey. J Pain Symptom Manage 1999; 18: 213-217. (chapter 1, 4)

Geertzen JH, de Bruijn H, de Bruijn-Kofman AT, Arendzen JH. Reflex sympathetic dystrophy: early treatment and psychological aspects. Arch Phys Med Rehabil 1994; 75: 442-446. (chapter 4)

Gheldof E, Crombez G, Van den Bussche E, Vinck J, Van Nieuwenhuyse A, Moens G, Mairiaux P, Vlaeyen JW. Pain-related fear predicts disability, but not pain severity: A path analytic approach of the fearavoidance model. Eur J Pain 2010; 14: 870. e1-9. (chapter 2)

Gieteling MJ, Bierma-Zeinstra SM, Passchier J, Berger MY. Prognosis of chronic or recurrent abdominal pain in children. J Pediatr Gastroenterol Nutr. 2008; 47: 316-326. (chapter 7) 
Goffaux P, Redmond WJ, Rainville P, Marchand S. Descending analgesia--when the spine echoes what the brain expects. Pain1 2007; 130: 137-143. (chapter 7)

Goossens ME, Vlaeyen JW, Hidding A, Kole-Snijders A, Evers SM. Treatment expectancy affects the outcome of cognitive-behavioral interventions in chronic pain. Clin J Pain1 2005; 21: 18-26; discussion 69-72. (chapter 7)

Goubert L, Crombez G, Vlaeyen JWS, Van Damme S, Van den Broeck A, Van Houdenhove B. The Tampa Scale for Kinesiophobia: Psychometric characteristics and standardization (translated from Dutch). Gedrag en Gezondheid 2000; 28: 54-62. (chapter 2, 6)

Goubert L, Francken G, Crombez G, Vansteenwegen D, Lysens R. Exposure to physical movement in chronic back pain patients: no evidence for generalization across different movements. Behav Res Ther 2002; 40: 415-429. (chapter 1, 4, 6)

Goubert L, Crombez G, Van Damme S, Vlaeyen JW, Bijttebier P, Roelofs J. Confirmatory factor analysis of the Tampa Scale for Kinesophobia: Invariant two-factor model across low back pain patients and fibromyalgia patients. Clin J Pain 2004; 20: 103-110. (chapter 1, 2, 3)

Goubert, L., Crombez, G., \& Lysens, R. (2005). Effects of varied-stimulus exposure on overpredictions of pain and behavioural performance in low back pain patients. Behav Res Ther 2005; 43: 1347-1361. (chapter 1,6)

Gunther LM, Denniston JC, Miller RR. Conducting exposure treatment in multiple contexts can prevent relapse. Behav Res Ther 1998; 36: 75-91. (chapter 7)

Harden RN. The rationale for integrated functional restoration. In: Wilson P, Stanton-Hicks M, Harden RN (Eds.). CRPS: Current Diagnosis and Therapy. Progress in Pain Research and Management vol. 32. Seattle; IASP Press 2005: 163-171. (chapter 4)

Hayes SC, Luoma JB, Bond FW, Masuda A, Lillis J. Acceptance and commitment therapy: model, processes and outcomes. Behav Res Ther 2006; 44: 1-25. (chapter 7)

Haythornthwaite JA, Clark MR, Pappagallo M, Raja SN. Pain coping strategies play a role in the persistence of pain in post-herpetic neuralgia. Pain 2003; 106: 453-460. (chapter 2)

Heneweer H, Aufdemkampe G, van Tulder MW, Kiers H, Stappaerts KH, Vanhees L. Psychosocial variables in patients with (sub)acute low back pain: an inception cohort in primary care physical therapy in The Netherlands. Spine 2007; 32: 586-592. (chapter 2)

Hermans D, Vansteenwegen D, Crombez G, Baeyens F, Eelen P. Expectancy-learning and evaluative learning in human classical conditioning: Affective priming as an indirect and unobstrusive measure of conditioned stimulus valence. Behav Res and Ther 2002a; 40: 217-234. (chapter 5, 6)

Hermans D, Crombez G, Vansteenwegen D, Baeyens F, Eelen P. Expectancy-learning and evaluative learning in human classical conditioning: differential effects of extinction. In Shohov SP (Ed.). Advances in Psychology Research vol. 12. New York; Nova Science Publishers 2002b: 17-41. (chapter 6)

Hermans D, Baeyens F, Eelen P. On the acguisition and activation of evaluative information in memory: evaluative learning and affective priming combined. In Musch J, Klauer KC (Eds.). The psychology of evaluation: affective processes in cognition and emotion. Mahwah, NJ; Lawrence Erlbaum 2003: 139168. (chapter 6)

Hermans D, Dirikx T, Vansteenwegen D, Baeyens F, Van den Bergh O, Eelen P. Reinstatement of fear responses in human aversive conditioning.Behav Res Ther1 2005; 43: 533-551. (chapter 1, 6)

Heuts PH, Vlaeyen JW, Roelofs J, de Bie RA, Aretz K, van Weel C, van Schayck OC. Pain-related fear and daily functioning in patients with osteoarthritis. Pain 2004; 110: 228-235. (chapter 1, 2, 3)

Hilz MJ, Devinsky O, Szczepanska H, Borod JC, Marthol H, Tutaj M. Right ventromedial prefrontal lesions result in paradoxical cardiovascular activation with emotional stimuli. Brain 2006; 129: 3343-3355. (chapter 7)

Hofmann SG. Self-focused attention before and after treatment of social phobia. Behav Res Ther1 2000; 38: 717-725. (chapter 1)

Hofmann SG. Cognitive mediation of treatment change in social phobia. J Consult Clin Psychol1 2004; 72 : 393-399. (chapter 1) 
Hofmann SG. Enhancing exposure-based therapy from translation research perspective. Behav Res Ther1 2007; 45: 1987-2001. (chapter 1)

Hout Van Den A, Vlaeyen JW, Heuts PH, Zijlema JH, Wijnen JA. Secondary prevention of work-related disability in nonspecific low back pain: Does problem-solving therapy help?: A randomized clinical trial. Clin J Pain 2003; 19: 87-96. (chapter 3)

Hsieh JC, Belfrage M, Stone-Elander S, Hansson P, Ingvar M. Central representation of chronic ongoing neuropathic pain studied by positron emission tomography. Pain1. 1995; 63: 225-236. (chapter 1)

Hurwitz EL, Carragee EJ, van der Velde G, Carroll LJ, Nordin M, Guzman J, Peloso PM, Holm LW, Côté P, Hogg-Johnson S, Cassidy JD, Haldeman S. Treatment of neck pain: noninvasive interventions: results of the Bone and Joint Decade 2000-2010 Task Force on Neck Pain and Its Associated Disorders. Spine 2008; 33: 123-152. (chapter 7)

Ingvar M. Pain and functional imaging. Philos Trans R Soc Lond B Biol Sci1 1999; 354: 1347-1358. (chapter 1)

Izard CE. Basic emotions, relations among emotions, and emotion-cognitive relations. Psychol Rev 1992; 99: 561-565. (chapter 1)

Jacobs WJ, Nadel L. Stress-induced recovery of fears and phobias. Psychol Rev 1985; 92 : 512-531. (chapter 1$)$

Jänig W, Baron R. Complex regional pain syndrome: mystery explained? Lancet Neurol 2003; 2: 687-697. (chapter 2, 4)

Jänig W, Baron R. Experimental approach to CRPS-I. Pain1 2004; 108: 3-7. (chapter 1)

Janssen R. Electronic Measurement Instrument: EMIUM. Department of Medical, Clinical and Experimental Psychology, University Maastricht, the Netherlands, 2005. (chapter 2)

Janssen SA, Arntz A, Bouts S. Anxiety and pain: epinephrine-induced hyperalgesia and attentional influences. Pain 1998; 76: 309-316. (chapter 4)

Jaspers JP. Whiplash and post-traumatic stress disorder. Dis Rehab 1998; 20: 397-404. (chapter 3)

Jelinek S, Germes D, Leyckes N, De Jong JR. The Photograph Series of Daily Activities (PHODA): Low Extremities. CD-rom Version 1.2, Hogeschool Zuyd, University Maastricht and Institute for Rehabilitation Research (iRv), The Netherlands, 2003. (chapter 1, 2, 4, 6)

Jensen MC, Brant-Zawadzki MN, Obuchowski N, Modic MT, Malkasian P, Ross JS. Magnetic resonance imaging of the lumbar spine in people without back pain. New England J Med 1994; 33: 69-73. (chapter 1,5$)$

Jensen MP, Turner JA, Romano JM. Changes in beliefs, catastrophizing, and coping are associated with improvement in multidisciplinary pain treatment. J Cons Clin Psychol 2001; 69: 655-662. (chapter 1 )

Juottonen K, Gockel M, Silen T, Hurri H, Hari R, Forss N. Altered central sensorimotor processing in patients with complex regional pain syndrome. Pain 1992; 98: 315-323. (chapter 4)

Katz J, Melzack R. Pain 'memories' in phantom limbs: review and clinical observations. Pain 1990; 43 : 319-336. (chapter 1)

Keefe F, Rumble ME, Scipio CD, Giodarno LA, Perri LCM. Psychological aspects of persistent pain: current state of the science. J Pain 2004; 5: 195-211. (chapter 1, 4, 6)

Keltner JR, Furst A, Fan C, Redfern R, Inglis B, Fields HL. Isolating the modulatory effect of expectation on pain transmission: a functional magnetic resonance imaging study. J Neurosci1 2006; 26: 4437-4443. (chapter 7)

Keogh E, Ellery D, Hunt C, Hannent I. Selective attentional bias for pain-related stimuli amongst pain fearful individuals. Pain 2001; 91: 91-100. (chapter 7)

Kerns RD, Rosenberg R, Jacob MC. Anger expression and chronic pain. J Behav Med 1994; 17: 57-67. (chapter 7)

Klenerman L, Slade PD, Stanley IM, Pennie B, Reilly JP, Atchison LE, Troup JD, Rose MJ. The prediction of chronicity in patients with an acute attack of low back pain in a general practice setting. Spine 1995; 20: 478-84. (chapter 5) 
Köke AJA, Heuts PHTG, Vlaeyen JWS, Weber WEJ. Meetinstrumenten chronische pijn: deel 1 functionele status. The Netherlands 1999; Pijn Kennis Centrum, academisch ziekenhuis Maastricht (azM). (chapter 3)

Kole-Snijders AM, Vlaeyen JW, Goossens ME, Rutten-van Molken MP, Heuts PH, van Breukelen G, van Eek $\mathrm{H}$. Chronic low back pain: What does cognitive coping skills training add to operant behavioral treatment? Results of a randomized clinical trial. J Consult Clin Psychol 1999 ; 67 : 931-944. (chapter 3)

Kopp B, Kunkel A, Muhlnickel W, Villringer K, Taub E, Flor H. Plasticity in the motor system related to therapy-induced improvement of movement after stroke. Neuro Report 1999; 10: 807-810. (chapter 4)

Kori SH, Miller RP, Todd DD. Kinesiophobia: A new view of chronic pain behaviour. Pain Management 1990; 3: 35-43. (chapter 1, 2, 3, 5)

Krause $\mathrm{P}$, Förderreuther $\mathrm{S}$, Straube A. TMS motor cortical brain mapping in patients with complex regional pain syndrome type I. Clin Neurophysiol1 2006; 117: 169-176. (chapter 7)

Kugler K, Wijn J, Geilen M, De Jong JR, Vlaeyen JWS. The Photograph series of Daily Activities (PHODA). CD-rom version 1.0: Institute for Rehabilitation Research and School for Physiotherapy Heerlen. The Netherlands, 1999. (chapter 1, 2, 5, 6)

Kvist J, Ek A, Sporrstedt K, Good L. Fearof re-injury: a hindrace for returning to sports after anterior cruciate ligament reconstruction. Knee Surg Sports Traumatol Arthrosc1 2005; 13: 393-397. (chapter 1)

Lang PJ. The emotion probe: Studies of motivation and attention. Am Psychol 1995; 50: 372-385. (chapter $2,7)$

Lang PJ, Bradley MM, Cuthbert BN. Emotion and motivation: measuring affective perception. J Clin Neurophysiol 1998; 15: 397-408. (chapter 4, 5)

Larsen DK, Taylor S, Asmundson GJ. Exploratory factor analysis of the pain anxiety symptoms scale in patients with chronic pain complaints. Pain 1997; 69: 27-34. (chapter 4)

Law M, Baptiste S, McColl M, Opzoomer A, Polatajko H, Pollock N. The Canadian Occupational Performance Measure: an outcome measure for Occupational Therapy. Can J Occup Ther 1990; 57: 82-87. (chapter 6)

Lee BH, Scharff L, Sethna NF, McCarthy CF, Scott-Sutherland J, Shea AM, Sullivan P, Meier P, Zurakowski $D$, Masek BJ, Berde CB. Physical therapy and cognitive-behavioral treatment for complex regional pain syndromes. J Pediatr 2002; 141: 135-140. (chapter 4)

Leeuw M, Goossens ME, Linton SJ, Crombez G, Boersma K, Vlaeyen JWS. The fear-avoidance model of musculoskeletal pain: Current state of scientific evidence. J Behav Med 2007a; 30: 77-94. (chapter 1, 2, 3)

Leeuw M, Goossens MEJB, van Breukelen G, Boersma K, Vlaeyen JWS. Measuring perceived harmfulness of physical activities in patients with chronic low back pain: The Photograph Series of Daily Activities - Short electronic Version. J Pain 2007b; 8: 840-849. (chapter 2, 6)

Leeuw M, Houben RMA, Severeijns R, Picavet HSJ, Schouten EGW, Vlaeyen JWS. Pain-related fear in low back pain: A prospective study in the general population. Eur J Pain 2007c; 11: 256-66. (chapter 2)

Leeuw M, Goossens MEJB, van Breukelen GJP, de Jong JR, Heuts PHTG, Smeets RJEM, Köke AJA, Vlaeyen JWS. Exposure in vivo versus operant graded activity in chronic low back pain patients: Results of a randomized controlled trial. Pain 2008; 138: 192-207. (chapter 1, 3, 6)

Lethem J, Slade PD, Troup JD, Bentley G. Outline of a fear-avoidance model of exaggerated pain perception - I. Behav Res Ther 1983; 21: 401-8. (chapter 1, 5)

Liepert J, Bauder H, Wolfgang HR, Miltner WH, Taub E, Weiller C. Treatment-induced cortical reorganization after stroke in humans. Stroke 2000; 31: 1210-1216. (chapter 4)

Lindström I, Ohlund C, Eek C, Wallin L, Peterson LE, Fordyce WE, Nachemson AL. The effect of graded activity on patients with subacute low back pain: a randomized prospective clinical study with an operant conditioning behavioral approach. Phys Ther 1992; 72: 279-90. (chapter 5) 
Linton SJ, Andersson T. Can chronic disability be prevented?: A randomized trial of a cognitive-behavior intervention and two forms of information for patients with spinal pain. Spine 2000a; 25: 28252831. (chapter 3)

Linton SJ. A review of psychological risk factors in back and neck pain. Spine 2000b; 25: 1148-1156. (chapter 2, 4, 5)

Linton SJ, Ryberg M. A cognitive-behavioral group intervention as prevention for persistent neck and back pain in a non-patient population: A randomized controlled trial. Pain 2001; 90: 83-90. (chapter 3)

Linton SJ, Vlaeyen J, Ostelo R. The back pain beliefs of health care providers: are we fear-avoidant? J Occup Rehabil1 2002; 12: 223-232. (chapter 1)

Linton SJ, Boersma K, Jansson M, Overmeer T, Lindblom K, Vlaeyen JW. A randomized controlled trial of exposure in vivo for patients with spinal pain reporting fear of work-related activities. Eur J Pain 2008; 12: 722-730. (chapter 1, 3, 6)

Lovibond PF, Shanks DR. The Role of Awareness in Pavlovian Conditioning: Empirical Evidence and Theoretical Implications. J Exp Psychol: Anim Behav Process 2002; 28: 3-26. (chapter 7)

Lorenz J, Hauck M, Paur RC, Nakamura Y, Zimmermann R, Bromm B, Engel AK. Cortical correlates of false expectations during pain intensity judgments--a possible manifestation of placebo/nocebo cognitions. Brain Behav Immun1 2005; 19: 283-295. (chapter 7)

Mahomed NN, Liang MH, Cook EF, Daltroy LH, Fortin PR, Fossel AH, Katz JN. The importance of patient expectations in predicting functional outcomes after total joint arthroplasty. J Rheumatol1 2002; 29 : 1273-1279. (chapter 7)

Maihöfner C, Forster C, Birklein F, Neundörfer B, Handwerker HO. Brain processing during mechanical hyperalgesia in complex regional pain syndrome: a functional MRI study. Pain1 2005; 114: 93-103. (chapter 7)

Maihöfner C, Baron R, DeCol R, Binder A, Birklein F, Deuschl G, Handwerker HO, Schattschneider J. The motor system shows adaptive changes in complex regional pain syndrome. Brain1 2007; 130: 26712687. (chapter 7)

Mannion AF, Muntener M, Taimela S, Dvorak J. A randomized clinical trial of three active therapies for chronic low back pain. Spine 1999; 24: 2435-2448. (chapter 3)

Mayou R. Medico-legal aspects of road traffic accidents. J Psychosom Res 1994; 38: 393-402. (chapter 3)

McCabe CS, Haigh RC, Ring EF, Halligan PW, Wall PD, Blake DR. A controlled pilot study of the utility of mirror visual feedback in the treatment of complex regional pain syndrome (type I). Rheumatology 2003; 42: 97-101. (chapter 4)

McCracken LM, Zayfert C, Gross RT. The Pain Anxiety Symptoms Scale: Development validation of a scale to measure fear of pain. Pain 1992; 50: 67-73. (chapter 3, 4, 5)

McCracken LM. "Attention" to pain in persons with chronic pain: a behavioral approach. Behav Ther 1997; 28: 271-81. (chapter 5)

McCracken LM, Spertus IL, Janeck AS, Sinclair D, Wetzel FT. Behavioral dimensions of adjustment in persons with chronic pain: Pain-related anxiety and acceptance. Pain 1999; 80: 283-290. (chapter 3, 4)

Melzack R. The McGill Pain Questionnaire: major properties and scoring methods. Pain 1975; 1: 277-299. (chapter 2)

Melzack RW, Wall PD. Pain mechanisms: A new theory. In: Steptoe A, Wardle J (Eds.). Psychosocial processes and health. New York, NY; Cambridge University Press 1994: 112-131. (chapter 1)

Melzack R, Israel R, Lacroix R, Schultz G. Phantom limbs in people with congenital limb deficiency or amputation in early childhood. Brain1 1997; 120: 1603-1620. (chapter 1)

Merskey $\mathrm{H}$, Bogduk K. Classification of chronic pain: definitions of chronic pain syndromes and definition of pain terms. Seatle; IASP Press 1994. (chapter 1, 6)

Mesulam MM. From sensation to cognition. Brain1 1998; 121: 1013-1052. (chapter 1)

Miller RP, Kori SH, Todd DD. The Tampa Scale for Kinisophobia. Unpublished Report, Tampa, FL; 1991. (chapter 1, 2, 4, 6) 
Mineka S, Henderson RW. Controllability and predictability in acquired motivation. Annu Rev Psychol1 1985; 36: 495-529. (chapter 1)

Mineka S, Mystkowski JL, Hladek D, Rodriguez BI. The effects of changing contexts on return of fear following exposure therapy for spider fear. J Consult Clin Psychol1 1999; 67: 599-604. (chapter 1)

Morley S. Single case research. In: Pary G, Watts FN (Eds.). Behavioural and Mental Health Research. A Handbook of Skills and Methods vol. 13 ( $2^{\text {nd }}$ ed.). Hove; Lawrence Erlbaum Press 1996: 277-314. (chapter 4)

Morley S, Eccleston C, Williams A. Systematic review and meta-analysis of randomized controlled trials of cognitive behavior therapy and behavior therapy for chronic pain in adults, excluding headache. Pain 1999; 80: 1-13. (chapter 3)

Morley S. Psychology of pain. Br J Anaesth1 2008; 101: 25-31. (chapter 1)

Moseley GL. Graded motor imagery is effective for long-standing complex regional pain syndrome: a randomized controlled trial. Pain 2004; 108: 192-198. (chapter 1, 4)

Moseley GL, Arntz A. The context of a noxious stimulus affects the pain it evokes. Pain 2007 ; 133 : 64-71. (chapter 1)

Moseley GL, Zalucki N, Birklein F, Marinus J, van Hilten JJ, Luomajoki H. Thinking About Movement Hurts: The Effect of Motor Imagery on Pain and Swelling in People With Chronic Arm Pain. Arthritis Rheum 2008; 59: 623-631. (chapter 2)

Mowrer $\mathrm{OH}$. A stimulus-response analysis of anxiety an dits role as a reinforcing agent. Psychol Rev $1939 ; 46: 553-565$. (chapter 1 )

Myers KM, Davis M. Behavioral and neural analysis of extinction. Neuron $2002 ; 36$ : 567-584. (chapter 1 )

Mystkowski JL, Craske MG, Echiverri AM. Treatment context and return of fear in spider phobia. Behav Ther 2002 ; 33 : 399-416. (chapter 1)

Mystkowski Jl, Mineka S, Vernon LL, Zinberg RE. Changes in caffeine states enhance return of fear in spider phobia. J Consult Clin Psychol $2003 ; 71: 243-250$. (chapter 1)

Nash JM, Williams DM, Nicholson R, Trask PC. The crontribution of pain-related anxiety to disability from headache. J Behav Med1 2006; 29: 61-67. (chapter 1)

Nederhand MJ, ljzerman MJ, Hermens HJ, Turk DC, Zilvold G. Predictive value of fear avoidance in developing chronic neck pain disability: Consequences for clinical decision making. Arch Phys Med Rehabil 2004; 85: 496-501. (chapter 3, 4)

Nederhand MJ, Hermens HJ, ljzerman MJ, Groothuis KGM, Turk DC. The effect of fear of movement on muscle activation in post-traumatic neck pain disability. Clin J Pain 2006; 22: 519-525. (chapter 3, 7)

Nelson DV. Treating patients with complex regional pain syndrome. In: Turk DC, Gatchel RJ (Eds.). Psychological Approaches to Pain Management. A Practitioner's Handbook (2nd ed.). New York; Guilford 2002: 470-488. (chapter 2, 4)

Neumann DL. The effects of physical context changes and multiple extinction contexts on two forms of renewal in a conditioned suppression task with humans. Learn Motivat 2006; 37: 149-175. (chapter 7)

Newman MG, Hofmann SG, Trabert Werner R, Walton T, Barr CT. Does behavioral treatment of social phobia lead to cognitive changes? Behav Ther 1994; 25: 503-517. (chapter 1)

Nicholas MK, Asghari A, Blyth FM. What do the numbers mean? Normative data in chronic pain measures. Pain 2008; 134: 158-173. (chapter 2)

Nieto R, Miró J, Huguet A. The fear-avoidance model in whiplash injuries. Eur J Pain1. 2009 May;13(5):518-23. (chapter 1)

Nijs J, Van Oosterwijck J, De Hertogh W. Rehabilitation of chronic whiplash: treatment of cervical dysfunctions or chronic pain syndrome? Clin Rheumatol 2009; 28: 243-251. (chapter 7)

Nisbett RE, Wilson TD. Telling More Than We Can Know: Verbal Reports on Mental Processes. Psychol Rev 1977; 84: 231-59. (chapter 5)

Oerlemans HM, Oostendorp RA, de Boo T, Goris RJ. Pain and reduced mobility in complex regional pain syndrome I: outcome of a prospective randomised controlled clinical trial of adjuvant physical therapy versus occupational therapy. Pain 1999; 83: 77-83. (chapter 4) 
Oerlemans HM, Oostendorp RA, de Boo T, van der Laan L, Severens JL, Goris JA. Adjuvant physical therapy versus occupational therapy in patients with reflex sympathetic dystrophy/complex regional pain syndrome type I. Arch Phys Med Rehabil 2000a; 81: 49-56. (chapter 4)

Oerlemans HM, Cup EHC, De Boo T, Goris RJA, Oostendorp RAB. The Radboud skills questionnaire: construction and reliability in patients with reflex sympathetic dystrophy of one upper extremity. Disab Rehab 2000b; 22: 233-245. (chapter 2, 6)

Öhman A, Mineka S. Fears, Phobias, and Preparedness: Toward an Evolved Module of Fear and Fear Learning. Psychol Rev 2001; 108: 483-522. (chapter 7)

Onghena P. Randomization tests for extensions and variations of $A B A B$ single-case experimental designs: A rejoinder. Behav Assess 1992; 14: 153-171. (chapter 3)

Onghena $\mathrm{P}$, Edgington ES. Randomization tests for restricted alternating treatments designs. Behav Res Ther 1994a; 32: 783-786. (chapter 4, 6)

Onghena P, Van Damme G. SCRT 1.1: Single-case randomization tests. Behav Res Meth Instrum Comput 1994b; 26: 369. (chapter 3, 4, 5, 6)

Onghena P, Edgington ES. Customization of pain treatments: Single-case design and analysis. Clin J Pain 2005; 21: 56-68. (chapter 3, 4, 6)

Osuch EA, Ketter TA, Kimbrell TA, George MS, Benson BE, Willis MW, Herscovitch P, Post RM. Regional cerebral metabolism associated with anxiety symptoms in affective disorder patients. Biol Psychiatry1 2000; 48: 1020-1023. (chapter 1)

Perez RS, Kwakkel G, Zuurmond WW, de Lange JJ. Treatment of reflex sympathetic dystrophy (CRPS type 1): a research synthesis of 21 randomized clinical trials. J Pain Symptom Manage 2001; 21: 511-526. (chapter 4)

Perez RS, Roorda LD, Zuurmond WW, Bannink II, Vranken JH, de Lange JJ. Measuring perceived activity limitations in lower extremity complex regional pain syndrome type 1 (CRPS I): test-retest reliability of two questionnaires. Clin Rehabil 2002; 16: 454-460. (chapter 4)

Perez RS, Collins S, Marinus J, Zuurmond WW, de Lange JJ. Diagnostic criteria for CRPS I: differences between patient profiles using three different diagnostic sets. Eur J Pain 2007; 11: 895-902. (chapter 2)

Persons JB, Silberschatz G. Are results of randomized controlled trials useful to psychotherapists? J Consult Clin Psychol 1998; 66: 126-135. (chapter 3, 6)

Peters ML, Vlaeyen JW, Kunnen AM. Is pain-related fear a predictor of somatosensory hypervigilance in chronic low back pain patients? Behav Res Ther 2002; 40: 85-103. (chapter 3)

Petrovic $P$, Ingvar M. Imaging cognitive modulation of pain processing. Pain1 2002; 95: 1-5. (chapter 1 )

Peyron R, Laurent B, García-Larrea L. Functional imaging of brain responses to pain. A review and metaanalysis (2000). Neurophysiol Clin1 2000; 30: 263-288. (chapter 1)

Philips HC. Avoidance behavior and its role in sustaining chronic pain. Behav Res Ther 1987; 25: 273-279. (chapter 1, 3)

Picavet HS, Vlaeyen JW, Schouten JS. Pain catastrophizing and kinesophobia: Predictors of chronic low back pain. Am J Epidemiol 2002; 156: 1028-1034. (chapter 3)

Porro CA, Cettolo V, Francescato MP, Baraldi P. Functional activity mapping of the mesial hemispheric wall during anticipation of pain. Neuroimage1 2003; 19: 1738-1747. (chapter 7)

Posner MI, Petersen SE. The attention system of the human brain. Anna rev Neurosci 1990; 13: 25-42. (chapter 1)

Price DD. Psychological and neural mechanisms of the affective dimension of pain. Science1 2000; 288: 1769-1772. (chapter 1)

Provinciali L, Baroni M, Illuminati L, Ceravolo MG. Multimodal treatment to prevent the late whiplash syndrome. Scan J Rehab Med 1996; 28: 105-111. (chapter 3)

Quirk GJ. Extinction: new excitement for an old phenomenon. Biol Psychiatry1 2006; 60: 317-318. (chapter 1$)$

Rachman SJ, Robinson S, Lopatha C. Is incomplete fear-reduction followed by a return of fear? Behav Res Ther 1987 ; 25 : 67-69. (chapter 6) 
Rachman S, Whittal M. The effect of an aversive event of the return of fear. Behav Res Ther 1989; 27 : 513-520. (chapter 3, 5)

Rachman SJ. The return of fear: review and prospect. Clin Psychol Rev 1989; 9: 513-520. (chapter 6)

Rachman S. A critique of cognitive therapy for anxiety disorders. J Behav Ther Exp Psychiatry 1993; 24: 279-288. (chapter 7)

Rachman S. The overprediction of fear: a review. Behav Res Ther 1994; 32: 691-700. (chapter 4)

Rachman S. A cognitive theory of obsessions: elaborations. Beh Res Ther 1998; 36: 385-401. (chapter 5)

Rainville J, Bagnall D, Phalen L. Health care providers' attitudes and beliefs about functional impairments and chronic back pain. Clin J Pain 1995; 11: 287-295. (chapter 3)

Rainville P, Bao QVH, Chrétien P. Pain-related emotions modulate experimental pain perception and automatic responses. Pain 2005; 118: 306-318. (chapter 3)

Ramachandran VS, Rogers-Ramachandran D, Cobb S. Touching the phantom limb. Nature 1995; 377 : 489-490. (chapter 4)

Rescorla RA, Herth CD. Reinstatement of fear to an extinguished conditioned stimulus. J Exp Psychol: Anim Behav Process 1975; 1: 88-96. (chapter 1, 6)

Rescorla RA. Preservation of Pavlovian associations through extinction. Quart J Exp Psychol 1996; 49B: 245-258. (chapter 1, 6)

Rescorla RA. Retraining of extinguished Pavlovian stimuli. J Exp Psychol: Anim Behav Process 2001; 27 : 115-124. (chapter 1)

Rescorla RA. Spontaneous recovery. Learn Mem 2004; 11: 501-509. (chapter 1)

Rescorla RA. Deepend extinction from compound stimulus presentation. J Exp Psychol: Anim Behav Process 2006; 32: 135-144. (chapter 1, 6)

Richlin DM, Carron H, Rowlingson JC, Sussman MD, Baugher WH, Goldner RD. Reflex sympathetic dystrophy: succesful treatment by transcutaneous nerve stimulation. J Pediatr 1978; 93: 84-86. (chapter 4)

Riedl B, Beckman T, Neundorfer B, Handwerker HO, Birklein F. Autonomic failure after stroke; is it indicative for pathophysiology of complex regional pain syndrome? Acta Neurol Scand 2001; 103: 27-34. (chapter 4)

Riddle DL, Stratford PW. Use of generic versus region-specific functional status measures on patients with cervical spine disorders. Phys Ther 1998; 78: 951-963. (chapter 3)

Risdon A, Eccleston C, Crombez G, McCracken L. How can we learn to live with pain? A Q-methodological analysis of the diverse understandings of acceptance of chronic pain. Soc Sci Med1 2003; 56: 375386. (chapter 1)

Rode S, Salkovskis PM, Jack T. An experimental study of attention, labelling and memory in people suffering from chronic pain. Pain 2001; 94: 193-203. (chapter 4)

Roelofs J, Peters ML, Vlaeyen JWS. Dutch version of the Pain Vigilance and Awareness Questionnaire: validity and reliability in a pain-free population. Behav Res Ther 2002; 40: 1081-1091. (chapter 5)

Roelofs J, Peters ML, van der Zijden M, Thielen FG, Vlaeyen JW. Selective attention and avoidance of pain-related stimuli: a dot-probe evaluation in a pain-free population. J Pain1 2003; 4: 322-328. (chapter 1)

Roelofs J, Goubert L, Peters ML, Vlaeyen JWS, Crombez G. The Tampa Scale for Kinesophobia: Further examination of psychometric properties in patients with chronic low back pain and fibromyalgia. Eur J Pain 2004; 8: 495-502. (chapter 3, 4, 6)

Roelofs J, Sluiter JK, Frings-Dresen MH, Goossens M, Thibault P, Boersma K, Vlaeyen JW. Fear of movement and (re)injury in chronic musculoskeletal pain: Evidence for an invariant two-factor model of the Tampa Scale for Kinesiophobia across pain diagnoses and Dutch, Swedish, and Canadian samples. Pain 2007; 131: 181-190. (chapter 2)

Roland M, Morris R. A study of the natural history of back pain: I. Development of a reliable and sensitive measure of disability in low back pain. Spine 1983; 8: 141-4. (chapter 5)

Roorda LD, Roebroeck ME, Lankhorst GJ, van Tilburg TG. The walking ability questionnaire: hierarchical scales to measure disabilities in rising and walking [in Dutch]. Revalidata 1996a; 18: 34-38. (chapter $2,4)$ 
Roorda LD, Roebroeck ME, Lankhorst GJ, van Tilburg TG, Bouter LM. Measuring functional limitations in rising and sitting down: development of a questionnaire. Arch Phys Med Rehabil 1996b; 77: 663669. (chapter 2, 4)

Roorda LD, Roebroeck ME, Lankhorst GJ, van Tilburg TG, Bouter LM. Measuring activity limitations in climbing stairs: development of a hierarchical scale for patients with lower extremity disorders living at home. Arch Phys Med Rehabil 2004; 85: 967-971. (chapter 4)

Rose MS, Klenerman L, Atchison L, Slade PD. An application of the fear avoidance model to three chronic pain problems. Behav res Ther $1992 ; 30: 359-365$. (chapter 1 )

Rosenfeld M, Seferiadis A, Carlsson J, Gunnarson R. Active intervention in patients with whiplashassociated disorders improves long-term prognosis: A randomized controlled clinical trial. Spine 2003; 28: 2491-2498. (chapter 3)

Rowe MK, Craske MG. Effects of an expanding-spaced vs massed exposure schedule on fear reduction and return of fear. Behav Res Ther 1998a ; 36 : 701-717. (chapter 6)

Rowe MK, Craske MG. Effects of varied-stimulus exposure training on fear reduction and return of fear. Behav Res Ther 1998b ; 36 : 719-734. (chapter 6)

Salkovskis PM, Clark DM, Hackmann A. Treatment of panic attacks using cognitive therapy without exposure or breathing retraining. Behav Res Ther 1991; 29: 161-166. (chapter 1)

Salkovskis PM, Clark DM. Cognitive therapy for panic attacks. J Cog Psychother 1991; 5: 215-226. (chapter 1)

Salkovskis PM, Bass C. In: Clark DM, Fairburn CG (Eds.). Science and practice of cognitive behaviour therapy. New York, NY; Oxford University Press 1997: 313-339. (chapter 1)

Salkovskis PM, Warwick HMC, Deale AC. Cognitive-Behavioral Treatment for Severe and Persistent Health Anxiety (Hypochondriasis). Brief Treatment and Crisis Intervention 2003; 3: 353-367. (chapter 7)

Sanders SH. Operant conditioning with chronic pain: Back to basic. In: Turk DC, Gatchel RJ (Eds.). Psychological Approaches to Pain Management: A Practitioner's Handbook (2nd ed.). New York, NY: Guilford 2002; 128-137. (chapter 3)

Sanbonmatsu DM, Fazio RH. The role of attitudes in memory-based decision making. J of Personality and Soc Psychol 1990; 59: 614-22. (chapter 5)

Sarafino EP. Health Psychology. Biopsychosocial Interactions. New York: Nohn Wiley \& Sons, Inc., 1998. (chapter 5)

Sawamoto N, Honda M, Okada T, Hanakawa T, Kanda M, Fukuyama H, Konishi J, Shibasaki H. Expectation of pain enhances responses to nonpainful somatosensory stimulation in the anterior cingulate cortex and parietal operculum/posterior insula: an event-related functional magnetic resonance imaging study. J Neurosci1 2000; 20: 7438-7445. (chapter 1)

Scharf MT, Woo NH, Lattal KM, Young JZ, Nguyen PV, Abel T. Protein synthesis is required for the enhancement of long-term potentiation and long-term memory by spaced training. J Neurophysiology 2002; 87: 2770-2777. (chapter 7)

Schasfoort FC, Bussman JB, Stam HJ. Ambulatory measurement of upper limb usage and mobility-related activities during normal daily life with an upper limb-activity monitor: a feasibility study. Med Biol Eng Comput 2002; 40: 173-182. (chapter 4)

Schasfoort FC, Bussmann JBJ, Zandbergen AMAJ, Stam HJ. Impact of upper limb complex regional pain syndrome type I on everyday life measured with a novel upper limb-activity monitor. Pain 2003; 101: 79-88. (chapter 4)

Schoenfeld-Smith K, Petroski GF, Hewett JE, Johnson JC, Wright GE, Smarr KL, Walker SE, Parker JC. A biopsychosocial model of disability in rheumatoid arthritis. Arthritis Care Res 1996; 9: 368-375. (chapter 7)

Schmidt RA, Bjork RA. New Conceptualizations of Practice: Common Principles in Three Paradigms Suggest New Concepts for Training. Psychol Science 1992; 3: 207-217. (chapter 7)

Schwartzman RJ, Kerrigan J. The movement disorder of reflex sympathetic dystrophy. Neurology 1990; 40: 57-61. (chapter 4) 
Schwenkreis P, Janssen F, Rommel O, Pleger B, Völker B, Hosbach I, Dertwinkel R, Maier C, Tegenthoff M. Bilateral motor cortex disinhibition in complex regional pain syndrome (CRPS) type I of the hand. Neurology1 2003; 61: 515-519. (chapter 7)

Schwoebel J, Friedman R, Duda N, Coslett HB. Pain and the body schema: evidence for peripheral effects on mental representations of movement. Brain 2001; 124: 2098-2104. (chapter 4)

Seminowicz DA, Davis KD. Cortical responses to pain in healthy individuals depends on pain catastrophizing. Pain 2006; 120: 297-306. (chapter 3)

Sgroi MI, Willebrand M, Ekselius L, Gerdin B, Andersson G. Fear-avoidance in recovered burn patients: Association with psychological and somatic symptoms. J Health Psychol 2005; 10: 491-502. (chapter 1, 3)

Sherman SJ, Rose JS, Koch K, Presson CC, Chassin L. Implicit and explicit attitudes toward cigarette smoking: The effects of context and motivation. J Soc Clin Psychol 2003; 22: 13-39. (chapter 5)

Sieben JM, Vlaeyen JWS, Tuerlinckx S, Portegijs P. Pain-related fear in acute low back pain: the first two weeks of a new episode. Eur J pain 2002; 6: 229-237. (chapter 5)

Sloan T, Telch MJ. The effects of safety-seeking behavior and guided threat reappraisal on fear reduction during exposure: An experimental investigation. Behav Res Ther 2002; 40: 235-251. (chapter 7)

Smeets RJ, Vlaeyen JW, Kester AD, Knottnerus JA. Reduction of pain catastrophizing mediates the outcome of both physical and cognitive-behavioral treatment in chronic low back pain. J Pain 2006a; 7 : 261-271. (chapter 1, 3)

Smeets RJ, Vlaeyen JW, Hidding A, Kester AD, van der Heijden GJ, van Geel AC, Knottnerus JA. Active rehabilitation for chronic low back pain: Cognitive-behavioral, physical, or both?: First direct posttreatment results from a randomized controlled trial. BMC Musculoskel Disord 2006; 7: 5. (chapter 3)

Smeets RJ, Beelen S, Goossens ME, Schouten EG, Knottnerus JA, Vlaeyen JW. Treatment expectancy and credibility are associated with the outcome of both physical and cognitive-behavioral treatment in chronic low back pain. Clin J Pain1 2008; 24: 305-315. (chapter 7)

Soderlund A, Lindberg P. An integrated physiotherapy/cognitive-behavioral approach to the analysis and treatment of chronic whiplash associated disorders, WAD. Disabil Rehabil 2001; 23: 436-447. (chapter 3)

Sotres-Bayon F, Cain CK, LeDoux JE. Brain Mechanisms of Fear Extinction: Historical Perspectives on the Contribution of Prefrontal Cortex. Biol Psychiatry 2006; 60: 329-336. (chapter 7)

Spinhoven P, Ter Kuile M, Kole-Snijders AM, Hutten Mansfeld M, Den Ouden DJ, Vlaeyen JW. Catastrophizing and internal pain control as mediators of outcome in the multidisciplinary treatment of chronic low back pain. Eur J Pain 2004; 8: 211-219. (chapter 1, 3)

Spitzer WO, Skovron ML, Salmi LR, Cassidy JD, Duranceau J, Suissa S, Zeiss E. Scientific monograph of the Quebec Task Force on Whiplash-Associated Disorders: Redefining "whiplash" and its management. Spine 1995; 20: 1S-73S. (chapter 3)

Stanton-Hicks M, Janig W, Hassenbusch S, Haddox JD, Boas R, Wilson P. Reflex sympathetic dystrophy: changing concepts and taxonomy. Pain 1995; 63: 127-133. (chapter 6)

Stanton-Hicks M, Baron R, Boas R, Gordh T, Harden N, Hendler N, Koltzenburg M, Raj P, Wilder R. Consensus report: complex regional pain syndromes: guidelines for therapy. Clin J Pain 1998; 14: 155166. (chapter 2)

Steketee G. Social support and treatment outcome of obsessive compulsive disorder at 9-month followup. Behav Psychother 1993; 21: 81-95. (chapter 1)

Sterner Y, Toolanen G, Knibestol M, Gerdle B, Hildingsson C. Prospective study of trigeminal sensibility after whiplash trauma. J Spinal Disord 2001; 14: 479-486. (chapter 3)

Stratford PW, Binkley JM, Riddle DL, Guyatt GH. Sensitivity to change of the Roland-Morris Back Pain Questionnaire: part 1. Phys Ther 1998; 78: 1186-1196. (chapter 5)

Stratford PW, Riddle DL, Binkley JM, Spadoni G, Westaway MD, Padfield B. Using the Neck Disability Index to make decisions concerning individual patients. Physiother Can 1999; 51: 107-112. (chapter 3) 
Sullivan MJ, Bishop SR, Pivik J. The pain catastrophizing scale: Development and validation. Psychol Assess 1995; 7: 524-532. (chapter 3, 4, 5, 6)

Sullivan MJL, Thorn BE, Haythornthwaite J, Keefe F, Martin M, Bradley L, Lefebvre JC. Theoretical perspectives on the relation between catastrophizing and pain. Clin J Pain 2001; 17: 52-64. (chapter 6)

Sullivan MJL, Lynch ME, Clark AJ. Dimensions of catastrophic thinking associated with pain experience and disability in patients with neuropathic pain conditions. Pain 2005; 113: 310-315. (chapter 6)

Sullivan MJ, Adams H, Horan S, Maher D, Boland D, Gross R. The role of perceived injustice in the experience of chronic pain and disability: scale development and validation. J Occup Rehabil1 2008; 18: 249-261. (chapter 7)

Swinkels-Meewisse IE, Roelofs J, Verbeek AL, Oostendorp RA, Vlaeyen JW. Fear of movement/(re)injury, disability and participation in acute low back pain. Pain 2003; 105: 371-379. (chapter 2)

Swinkels-Meewisse IE, Roelofs J, Oostendorp RA, Verbeek AL, Vlaeyen JW. Acute low back pain: Painrelated fear and pain catastrophizing influence physical performance and perceived disability. Pain 2006; 120: 36-43. (chapter 3)

Tang NK, Salkovskis PM, Poplavskaya E, Wright KJ, Hanna M, Hester J. Increased use of safety-seeking behaviors in chronic back pain patients with high health anxiety. Behav Res Ther1 2007; 45: 28212835. (chapter 1)

Thwaites R, Freeston MH. Safety-Seeking Behaviours: Fact or Function? How Can We Clinically Differentiate Between Safety Behaviours and Adaptive Coping Strategies Across Anxiety Disorders? Behav Cogn Psychother 2005; 33: 177-188. (chapter 7)1

Tracey KJ. The inflammatory reflex. Nature1 2002; 420: 853-859. (chapter 7)

Trost Z, France CR, Thomas JS. Exposure to movement in chronic back pain: Evidence of successful generalization across a reaching task. Pain 2008; 137: 26-33. (chapter 1, 6)

Trost Z, France CR, Thomas JS. Examination of the photograph series of daily activities (phoda) scale in chronic low back pain patients with high and low kinesiophobia. Pain 2009; 141: 276-282. (chapter 2)

Turk DC. Customizing treatment for chronic pain patients: who, what and why. Clin J Pain 1990; 6: 255270. (chapter 1)

Turk DC, Okifuji A. Perception of traumatic onset, compensation status, and physical findings: impact on pain severity, emotional distress, and disability in chronic pain patients. J Behav Med1 1996; 19: 435453. (chapter 7)

Turk DC, Okifuji A. Psychological factors in chronic pain: evolution and revolution. J Consult Clin Psychol 2002; 70: 678-690. (chapter 2)

Turk DC, Robinson JP, Burwinkle T. Prevalence of fear of pain and activity in patients with fibromyalgia syndrome. J Pain1 2004; 5: 483-490. (chapter 1)

Turk DC, Robinson JP, Sherman JJ, Burwinkle T, Swanson K. Assessing fear in patients with cervical pain: development and validation of the Pictorial Fear of Activity Scale-Cervical (PFActS-C). Pain1 2008; 139: 55-62. (chapter 1)

Turner JA, Franklin G, Fulton-Kehoe D, Sheppard L, Wickizer TM, Wu R, Gluck JV, Egan K. Worker recovery expectations and fear-avoidance predict work disability in a population-based workers' compensation back pain sample. Spine 2006; 31: 682-689. (chapter 3)

Turner JA, Holtzman S, Mancl L. Mediators, moderators, and predictors of therapeutic change in cognitive-behavioral therapy for chronic pain. Pain1 2007; 127: 276-286. (chapter 1)

Van Damme S, Crombez G, Bijttebier P, Goubert L, Van Houdenhove B. A confirmatory factor analysis of the Pain Catastrophizing Scale: Invariant factor structure across clinical and non-clinical populations. Pain 2002; 96: 319-324. (chapter 3, 6)

Van Damme S, Crombez G, Eccleston C. Disengagement from pain: the role of catastrophic thinking about pain. Pain1 2004; 107: 70-76. (chapter 1)

Van Damme S, Crombez G, Hermans D, Koster EH, Eccleston C. The role of extinction and reinstatement in attentional bias to threat: a conditioning approach. Behav Res Ther1 2006; 44: 1555-1563. (chapter 1) 
Van der Zee KI, Sanderman R. Het meten van de algemene gezondheidstoestand met de RAND-36. Een handleiding. Groningen: Noordelijk Centrum voor Gezondheidsvraagstukken; 1993. (chapter 2)

Vangronsveld KL, Peters M, Goossens M, Vlaeyen J. The influence of fear of movement and pain catastrophizing on daily pain and disability in individuals with acute whiplash injury: a daily diary study. Pain 2008; 139: 449-457. (chapter 2)1

Vansteenwegen D, Vervliet B, Hermans D, Beckers T, Baeyens F, Eelen P. Stronger renewal in human fear conditioning when tested with an acquisition retrieval cue than with an extinction retrieval cue. Behav Res Ther 2006; 44: 1717-1725. (chapter 7)1

Vansteenwegen D, Vervliet B, Iberico C, Baeyens F, Van den Bergh O, Hermans D. The repeated confrontation with videotapes of spiders in multiple contexts attenuates renewal of fear in spider-anxious students. Behav Res Ther 2007; 45: 1169-1179. (chapter 1, 3, 6)

Vase L, Robinson ME, Verne GN, Price DD. Increased placebo analgesia over time in irritable bowel syndrome (IBS) patients is associated with desire and expectation but not endogenous opioid mechanisms. Pain1 2005; 115: 338-347. (chapter 7)

Vassiliou T, Kaluza G, Putzke C, Wulf H, Schnabel M. Physical therapy and active exercises: An adequate treatment for prevention of late whiplash syndrome?: Randomized controlled trial in 200 patients. Pain 2006; 124: 69-76. (chapter 3)

Veldman PH, Reynen HM, Arntz IE, Goris RJ. Signs and symptoms of reflex sympathetic dystrophy: prospective study of 829 patients. Lancet 1993; 342: 1012-1016. (chapter 2, 4).

Vendrig AA, van Akkerveeken PF, McWhorter KR. Results of a multimodal treatment program for patients with chronic symptoms after a whiplash injury of the neck. Spine 2000; 25: 238-244. (chapter 3)

Verbunt JA, Seelen HA, Vlaeyen JW, van de Heijden GJ, Heuts PH, Pons K, Knottnerus JA. Disuse and deconditioning in chronic low back pain: concepts and hypotheses on contributing mechanisms. Eur J Pain 2003; 7: 9-21. (chapter 2)

Vernon H, Mior S. The Neck Disability Index: A study of reliability and validity. J Manip Physiol Ther 1991; 14: 409-415. (chapter 3)

Vlaeyen JWS, Kole-Snijders AMJ, Boeren RGB, van Eek H. Fear of movement/(re)injury in chronic low back pain and its relation to behavioral performance. Pain 1995a; 62: 363-372. (chapter 1, 2, 3, 4, 5, 6)

Vlaeyen JWS, Kole-Snijders AMJ, Rotteveel A, Ruesink R, Heuts PHTG. The role of fear of movement/(re)injury in pain disability. J Occup Rehabil 1995b; 5: 235-252. (chapter 3, 2, 4, 5, 6)

Vlaeyen JWS, Linton SJ. Fear-avoidance and its consequences in chronic musculoskeletal pain: A state of the art. Pain 2000; 85: 317-332. (chapter 1, 2, 3, 4, 5, 6)

Vlaeyen JWS, De Jong JR, Geilen M, Heuts PHTG, van Breukelen G. Graded exposure in vivo in the treatment of pain-related fear: A replicated single-case experimental design in four patients with chronic low back pain. Behav Res Ther 2001; 39: 151-166. (chapter 1, 3, 4, 5, 6)

Vlaeyen JWS, De Jong JR, Geilen M, Heuts PHTG, van Breukelen G. The treatment of fear of movement/(re)injury in chronic low back pain: Further evidence on the effectiveness of exposure in vivo. Clin J Pain 2002a; 18: 251-261. (chapter 1, 3, 4, 5, 6)

Vlaeyen JWS, De Jong JR, Onghena P, Kerckhoffs-Hanssen M, Kole-Snijders AM. Can pain-related fear be reduced?: The application of cognitive-behavioral exposure in vivo. Pain Res Manag 2002b; 7: 144153. (chapter 1, 3, 4, 5, 6)

Vlaeyen JWS, De Jong JR, Sieben JM, Crombez G. Graded exposure in vivo for pain-related fear. In: Turk DC, Gatchel RJ (Eds.). Psychological Approaches to Pain Management: A Practitioner's Handbook (2nd edition). New York, NY: Guilford 2002c; 210-233. (chapter 1, 3, 4, 5, 6)

Vlaeyen JWS, De Jong JR, Leeuw M, Crombez G. Fear reduction in chronic pain: Graded exposure in vivo with behavioral experiments. In: Asmundson GJG, Vlaeyen JWS, Crombez G (Eds.). Understanding and Treating Fear of Pain. New York: Oxford University Press 2004; 313-346. (chapter 1, 2, 3, 6)

Vlaeyen JW, Morley S. Cognitive-behavioral treatments for chronic pain: what works for whom? Clin J Pain1 2005; 21: 1-8. (chapter 1, 2) 
Vos CJ, Verhagen AP, Koes BW. Reliability and responsiveness of the Dutch version of the Neck Disability Index in patients with acute neck pain in general practice. Eur Spine J 2006; 15: 1729-1736. (chapter 3)

Waddell G, Newton M, Henderson I, Somerville D, Main CJ. A Fear-Avoidance Beliefs Questionnaire (FABQ) and the role of fear-avoidance beliefs in chronic low back pain and disability. Pain 1993; 52 : 157-68. (chapter 1, 5)

Waddell G. The Back Pain Revolution, 2nd ed. Edinburgh: Churchill Livingstone; 2004. (chapter 1)

Wade SL, Monroe SM, Michelson LK. Chronic life stress and treatment outcome in agoraphobia with panic attacks. Am J Psychiatry 1993; 150: 1491-1495. (chapter 1)

Wagner AR, Brandon SE. Evolution of a structured connectionist model of Pavlovian conditioning (AESOP). In: Klein SB, Mowrer RR (Eds.). Contemporary learning theories: Pavlovian conditioning and the status of traditional learning theory. Hillsdale, NJ, England; Lawrence Erlbaum Associates, Inc, 1989: 149-189. (chapter 1)

Wampold BE, Furlong MJ. The heuristics of visual inference. Behav Assess 1981; 3: 79-92. (chapter 3, 4)

Wampold BE. Outcomes of individual counseling and psychotherapy: Empirical evidence addressing two fundamental questions. In: Brown SD, Lent RW (Eds.). Handbook of counseling psychology (3rd ed.). Hoboken, NJ: John Wiley \& Sons Inc, 2000: 711-739. (chapter 1)

Wampold BE. Contextualizing psychotherapy as a healing practice: Culture, history, and methods. Appl Prev Psychol 2001; 10: 69-86. (chapter 7)

Ware JE, Sherbourne CD. The MOS 36-Item Short-Form Health Survey (SF-36). Medical Care 1992; 30: 473-83. (chapter 2)

Watkins LR, Maier SF. Beyond neurons: evidence that immune and glial cells contribute to pathological pain states. Physiol Rev1 2002; 82: 981-1011. (chapter 7)

Welk GJ, Schaben JA, Morrow Jr JR. Reliability of accelerometry-based activity monitors: A generalizability study. Med Sci Sports Exerc 2004; 36: 1637-1645. (chapter 3)

Wessa M, Karl A, Flor H. Central and peripheral psychophysiological responses to trauma-related cues in subclinical posttraumatic stress disorder: A pilot study. Exp Brain Res 2005; 167: 56-65. (chapter 7)

Wicksell RK, Ahlqvist J, Bring A, Melin L, Olsson GL. Can exposure and acceptance strategies improve functioning and life satisfaction in people with chronic pain and whiplash-associated disorders (WAD)? A randomized controlled trial. Cogn Behav Ther 2008; 37: 1-14. (chapter 7)

Wilson TD, Lindsey S, Schooler TY. A model of dual attitudes. Psychol Rev 2000; 107: 101-126. (chapter 5)

Wilson PR, Stanton-Hicks M, Harden NR. CRPS: Current Diagnosis and Therapy. Progress in Pain Research and Management, vol. 22. Seattle: IASP Press; 2005. (chapter 2)

Woby SR, Watson PJ, Roach NK, Urmston M. Are changes in fear-avoidance beliefs, catastrophizing, and appraisals of control, predictive of changes in chronic low back pain and disability? Eur J Pain1 2004; 8: 201-210. (chapter 1)

Wolpe J. Psychotherapy by Reciprocal Inhibition. Stanford: Stanford University Press, CA; 1958. (chapter 5)

Woods MP, Asmundson GJG. Evaluating the efficacy of graded in vivo exposure for the treatment of fear in patients with chronic back pain: A randomized controlled clinical trial. Pain 2008; 136: 271-280. (chapter 1, 3, 6) 


\section{Dankwoord \\ (Words of thanks)}


Het schrijven van een proefschrift was naast een klinische taak en het vaderschap een zware exercitie. Zonder de steun en hulp van vele lieve mensen was dit nooit gelukt. Het is dan ook fijn om iedereen die direct of indirect heeft bijgedragen aan dit proefschrift hieronder te kunnen bedanken.

Ik kan niet anders concluderen dat ik bevoorrecht ben geweest om onder de vleugels van Johan Vlaeyen te hebben mogen werken aan dit proefschrift. Johan, een betere promotor en inspirator was niet voor te stellen. Je passie en inzet voor het onderzoek dat we samen hebben uitgevoerd, en nog steeds uitvoeren, is bewonderenswaardig. Ondanks je overvolle agenda, maakte je tijd voor mij vrij, en werd ik iedere keer weer verrast door de snelheid waarmee ik teksten voorzien van commentaar terugkreeg. De ruimte die ik kreeg voor mijn eigen inbreng en de waardering voor mij als persoon heeft me veel zelfvertrouwen gegeven. Dankzij jou heb ik niet alleen veel geleerd over pijn, wetenschappelijk onderzoek en de gedragstherapie, maar ook over mezelf. Prioriteiten stellen! Johan, hopelijk is dit proefschrift slechts een tussenstation en zullen we op velerlei levensgebieden nog samen genieten.

De leden van de beoordelingscommissie: Madelon Peters (voorzitter), Rob Smeets, Maarten van Kleef, Steven Linton en Deborah Vansteenwegen dank ik voor het beoordelen van mijn proefschrift. Rob, ook bedankt voor het kritisch nakijken van mijn proefschrift op (taalkundige) foutjes!

Dank gaat uit naar de fantastische inzet en medewerking van de behandelteams en de patiënten tijdens de studies van dit proefschrift. Mede dankzij Adelante afdeling 'Reuma en Pijn' zijn de eerste exposure behandelingen opgezet en bestudeerd. Vooral Peter Heuts, als toenmalig hoofd, ben ik hier zeer dankbaar voor. Het klinisch deel van de exposure therapie werd bij Adelante mede uitgevoerd door Mario Geilen en Herman Mulder. Mario en Herman, bedankt voor de samenwerking en het feit dat jullie jezelf ook hebben blootgesteld aan het geven van een behandeling die niet overeenstemde met de reguliere zorg.

De samenwerking met mijn huidige collega's van de afdeling Revalidatie MUMC/azM is van grote waarde. Niet alleen voor de totstandkoming van dit proefschrift, maar ook voor het met heel veel plezier initiëren en uitvoeren van pijnrevalidatie en pijnonderzoek. Joop Ruijgrok, revalidatiearts en ad interim-hoofd, bedankt voor je inzet bij het creëren van mijn positie binnen de afdeling Revalidatie. Dankzij jou heeft de exposure behandeling een belangrijke plek binnen de klinische zorg gekregen. Rob Smeets, huidig hoofd, dank ik voor het geven van de ruimte om mijn onderzoeksactiviteiten te kunnen blijven uitvoeren binnen de nieuwe vakgroep Revalidatiegeneeskunde van de UM. Ook Jeanine Verbunt, revalidatiearts, wil ik 
bedanken voor haar steun hierbij. Rob, Joop en Jeanine en de revalidatieartsen Marjon van Eijsden, Erik de Klerk, Joep Cluitmans en Antal Sanders, bedankt voor jullie inzet bij het exposure onderzoek. En nu de paramedici: enthousiast en vol met energie! Corine Cuypers, op de eerste plaats wil ik jou bedanken voor je inzet. Je rol binnen de exposure behandeling en je hulp bij organisatorische zaken rondom exposure verdienen veel lof. Maria Kerkhofs, als eerste paramedicus binnen het MUMC/azM heb jij je 'gewaagd' aan het geven van exposure. Hiermee heb je je collega's laten inzien dat het mogelijk is om je eigen angst als behandelaar te overwinnen; bedankt! Anita Stevens, Christoph Loo, Marianne Maas, Margot van Melick, Tanja Hermans en Corien Rebel, ook jullie bedankt voor je inzet.

Naast de hierboven genoemde collega's van het MUMC/azM wil ik in het bijzonder Marlies den Hollander en Els Oostveen bedanken. Marlies, zowel binnen het onderzoek als de patiëntenzorg heb je me altijd op een positieve wijze bijgestaan en van suggesties voorzien. Door jouw enthousiasme en inzet heeft de exposure behandeling aan kwaliteit gewonnen. Ik geef nu het promotiestokje door aan jou! Els, jij hebt voor mij op de afdeling revalidatie van het MUMC/azM de randvoorwaarden gecreeerd waardoor ik mijn werk als gedragstherapeut/onderzoeker met zoveel plezier kan uitvoeren. Altijd kan ik een beroep op je doen en sta je voor mij klaar. Bedankt voor al deze goede zorgen! Marlies en Els, ik ben in mijn nopjes dat jullie als paranimfen tijdens de promotieplechtigheid aan mijn zijde staan!

Sjors Roox, hoofd van de afdeling fysiotherapie MUMC/azM, en Phil Geerlings, hoofd van de afdeling ergotherapie MUMC/azM, dank ik dat zij behandelaars van hun afdeling de kans hebben gegeven mee te werken aan het exposure onderzoek.

De samenwerking met de poli pijnbestrijding MUMC/azM is van groot belang geweest voor het exposure onderzoek. Maarten van Kleef, afdelingshoofd, en Jaap Patijn, coördinator pijnbestrijding, bedankt voor jullie inzet.

Het promotieonderzoek was ondergebracht bij het departement Clinical Psychological Science (CPS) van de Faculty of Psychology and Neuroscience UM, voorheen Departement Medische Klinische en Experimentele Psychologie (DMKEP) van de Faculty of Health, Medicine and Life Sciences UM, en de Nederlands-Vlaamse onderzoekschool Experimentele Psychologie (EPP). Angela Verweij, dankzij jouw inzet voor prima werkfaciliteiten en aandacht voor sociale aspecten heb ik mijn verblijf bij DMKEP/CPS als zeer prettig ervaren. Bedankt hiervoor! Arnoud Arntz en Rense Hoekstra, bedankt voor jullie inzet om mij te ontlasten van patiëntenzorg zodat ik een aaneengesloten periode heb kunnen werken aan mijn proefschrift. Erik Schouten, bedankt voor je statistische ondersteuning en Rosanne Janssen, bedankt voor je hulp bij het opslaan en verwerken van de onderzoeksdata. Leve EMIUM! Tot slot 
wil ik de collega's Mariëlle Goossens, Madelon Peters en Sita van Riet, en de voormalig DMKEP/CPS collega's Jantha de Gelder, Maaike Leeuw, Karolien Vangronsveld, Anja van den Hout en Peter de Jong bedanken voor hun hulp en/of advies bij de verschillende studies die zijn uitgevoerd.

Peter Boon, Ruud Severeijns, Gerdy Konings en Lidwine Bruinen, alle werkzaam bij de afdeling Medische Psychologie MUMC/azM ten tijde van mijn aanstelling daar, bedankt voor jullie inzet en hulp. Peter Boon heeft me de mogelijkheid gegeven om de opleiding tot gedragstherapeut te volgen en onderzoek te verrichten. Ruud en Gerdy, jullie intervisie heeft aan de basis gestaan van mijn ontwikkeling tot gedragstherapeut.

Dankzij Patrick Onghena en Isis Bulté, beide werkzaam bij de faculteit Psychologie en Pedagogische Wetenschappen Katholieke Universiteit Leuven, heb ik ervaren en geleerd dat de gedragswetenschappen en de revalidatiegeneeskunde niet zonder single-case studies kan. De kennis en informatie die je hiermee opdoet is van onschatbare waarde voor zowel de onderzoeker als de cliënt/patiënt. Patrick en Isis, bedankt voor jullie hulp en advies bij de opzet van de single-case studies en het statistisch analyseren van de data.

During my PhD period I had the good fortune to be introduced by Johan Vlaeyen to many foreign colleagues. The exchange of exposure experience with Steven Linton, Katja Boersma and colleagues of the School of Law, Psychology and Social work at the Örebro University, Sweden, was very instructive in particular. Steven, I am honoured that you will be one of the members of the corona during the PhD ceremony. I also thank Stephen Morley, Faculty of Health and Medicine at the University of Leeds, United Kingdom, for his valuable discussions.

Coen en Joke, mijn ouders. Van tennisser tot wetenschapper, gelukkig hebben jullie je hierbij neergelegd. Coen, misschien heb ik onbewust jouw levensmotto gevolgd: gewoon doen!

Ity en Hay, mijn schoonouders. Nooit is het teveel, altijd staan jullie klaar! Zonder jullie ondersteuning was het eind van dit proefschrift nog lang niet in zicht geweest. Ik mag me gelukkig prijzen.

De meest dierbare en de allerliefste schatten bewaar ik voor het laatst: Mariëlle, Job, Tom en Pien. Mariëlle niet alleen op het gebied van onderzoek ben jij een enorme steun geweest, ook in ons privéleven is het een groot feest om samen met jou te zijn. Vol bewondering zie ik jou door het leven gaan: drie kinderen, werk, hobby's en een partner die soms weinig bewust is van zijn eigen handelingen en het 
effect van zijn mededelingen, argeloos is en daardoor eenvoudig te verleiden. Respect! Job, Tom en Pien, ik ben zo trots als een pauw op jullie alledrie. Het plezier dat jullie me geven, maakt het leven tot een waar genot en zorgt ervoor dat iedere hindernis als sneeuw voor de zon verdwijnt. 

About the author 
Jeroen de Jong was born on 1 May, 1970. In 1988 he obtained his secondary school diploma at 'Het Zuidlimburgs Avondcollege' in Heerlen.

\section{Main research activities}

After graduation from the VU University Amsterdam, Faculty of Human Movement Sciences (op internet gevonden, maar het kan zijn dat het vroeger anders heette) in 1994 and during the graduation of Mental Healthat Maastricht University, Faculty of Health, Medicine and Life Sciences, between 1995-1997 Jeroen started his research career at the Department of Rheumatology and Pain of Adelante, Hoensbroek, the Netherlands, in close collaboration with the Institute for Rehabilitation Research, Hoensbroek, the Netherlands and Maastricht University, the Netherlands. Research was focused on the effectiveness of cognitive-behavioral treatment programs for patients with chronic low back pain and carried out by an interdisciplinary team. From 2000 Jeroen's research career continued at the Department of Medical Clinical and Experimental Psychology, now called Clinical Psychological Science, Faculty of Psychology and Neuroscience, Maastricht University, the Netherlands. From then on research activities have included the development and psychometric analysis of pain assessment instruments, laboratory studies focusing on the role of pain-related fear, and clinical trials and single-case studies examining differential effectiveness of cognitive-behavioral treatments in patients with musculoskeletal, work-related and neuropathic pain, which were labelled to the School of Experimental Psychology (EPP). Research in this area is often carried out with international colleagues. Together with Prof. dr. Johan Vlaeyen, Jeroen is recognized as the founder of the Graded Exposure in Vivo therapy in chronic pain. This therapy is also the central issue of this thesis. Currently, research is also focused on the mechanism behind the success of Graded Exposure in Vivo by means of fMRI studies. Furthermore, Jeroen has designed a new 'Stop-Rule-Management program' for patients with Complaints of Arm Neck and/or Shoulder (CANS). The effectiveness of this program is still being examined. Since 2010 has Jeroen worked for the Department of Rehabilitation Medicine at Maastricht University and all research activities have been labelled to the School for Public Health and Primary Care (CAPHRI).

\section{Regular teaching}

Jeroen has an active role in the education of medicine at Maastricht University. Besides lectures and coaching work he also provides practical exercises on chronic pain and rehabilitation. Jeroen is often asked to give lectures or workshops about Graded Exposure in Vivo in chronic pain by his (inter)national colleagues and for congresses/symposia. 


\section{Main clinical work}

During his postgraduate education in Behavior Therapy (2000-2004) Jeroen worked as a Kinesiologist / Pain Therapist / Behavior Therapist in training at Hoensbroek Rehabilitation Centre (currently Adelante) Department of Rheumatism \& Pain and University Hospital Maastricht Department of Medical Psychology. After finishing his postgraduate education in Behavior Therapy in 2004 Jeroen started working as a Behavior Therapist / Kinesiologist / Pain Therapist at the Department of Rehabilitation University Hospital Maastricht. Within this position Jeroen has developed current outpatient rehabilitation for chronic pain and unexplained physical complaints. He also trains the therapists and acts as supervisor.

\section{Goals/ambitions of goal/ambition}

Jeroen's ambiton is to be a good father, therapist and researcher, and to make an important contribution to rehabilitation medicine.

\section{Quote}

Pain is inevitable. Suffering is optional. 



\section{Publications}




\section{Cat.: International SCl or SSCl indexed.}

De Peuter S, De Jong JR, Crombez G, Vlaeyen JWS. The nature and treatment of pain-related fear in chronic musculoskeletal pain. Journal of Cognitive Psychotherapy 2009; 23: 85-103.

De Jong JR, Vangronsveld K, Peters ML, Goossens ME, Onghena P, Bulté I, Vlaeyen JW. Reduction of pain-related fear and disability in post-traumatic neck pain: a replicated single-case experimental study of exposure in vivo. Journal of Pain 2008; 9: 1123-1134.

Leeuw M, Goossens ME, van Breukelen GJ, De Jong JR, Heuts PH, Smeets RJ, Köke AJ, Vlaeyen JW. Exposure in vivo versus operant graded activity in chronic low back pain patients: results of a randomized controlled trial. Pain 2008; 138: 192-207.

De Jong JR, Vlaeyen JWS, Onghena P, Cuypers C, den Hollander M, Ruygrok JM. Pain-related fear in Complex Regional Pain Syndrome type I: The application of graded exposure in vivo. Pain 2005; 116: 264-275.

Jeroen R de Jong, Johan WS Vlaeyen, Patrick Onghena, Mariëlle EJB Goossens, Mario Geilen, Herman Mulder. Fear of movement/(re) injury in chronic low back pain: Education or exposure in vivo as mediator to fear reduction? Clinical Journal of Pain 2005: 21(1); 9-17.

Boersma K, Linton SJ, Overmeer T, Vlaeyen J, de Jong JR. Lowering fear-avoidance and enhancing function through exposure in vivo. A multiple baseline study across six patients with back pain. Pain 2004: 108; 8-16.

Vlaeyen JW, de Jong JR, Geilen M., Heuts PH, van Breukelen G. The treatment of fear of movement/(re)injury in chronic low back pain: further evidence on the effectiveness of exposure in vivo. Clinical Journal of Pain 2002: 18; 251-61.

Vlaeyen JW, de Jong JR, Onghena P, Kerckhoffs-Hanssen M, Kole-Snijders A.M. Can pain-related fear be reduced? The application of cognitive- behavioral exposure in vivo. Pain Research and Management 2002: 7; 144-153.

Roelofs J, Boissevin MD, Peters ML, de Jong JR, Vlaeyen JW. Psychological treatments for chronic low back pain: past, present and beyond. Pain Reviews 2002: 9; 29-40. 
Linton SJ, Overmeer T, Janson M, Vlaeyen JWS, de Jong JR. Graded in vivo exposure treatment for fear-avoidant pain patients with functional disability: a case study. Cognitive Behaviour Therapy 2002: 31; 49-58.

Vlaeyen JWS, de Jong JR, Geilen M, Heuts PHTG, van Breukelen G. Graded exposure in vivo in the treatment of pain-related fear. A replicated single-case experimental design in four patients with chronic low back pain. Behaviour Research and Therapy 2001: 39; 151-166.

\section{Cat.: National}

Mulders D, Egger J, De Jong JR, Arntz A. Dialectische gedragstherapie voor de behandeling van BPS. Het grillige en langdurige beloop van parasuïcide. Tijdschrift voor Psychotherapie 2010: 3; 190-203.

Ruijgrok JM, De Jong JR. Macedonië in de polder. Medisch Contact 2005: 60(4); 160 162.

De Jong JR. Chronische pijn vanuit een biopsychosociaal perspectief; implicaties voor behandelingen en de eerste bevindingen bij patiënten met CRPS-I. Nieuwsbrief Nederlandse Vereniging van Posttraumatische Dystrofie Patiënten 2004: 15(2); 4-7.

Vlaeyen JW, Peters ML, Roelofs J, De Jong JR, Sieben J, Houben R, Verbunt J, Lamoth C. Serie onderzoek en psychotherapie: Catastrofale misinterpretaties, vrees voor beweging, letsel en pijn bij lage rugpijn. Tijdschrift voor Psychotherapie 2002: 28(3); 205-222.

De Jong JR, Goubert L, Vlaeyen J, Crombez G. Exposure in vivo bij chronische lagerugpijn. Gedragstherapie 2002: 35; 49-70.

De Jong JR, Vlaeyen JWS, Geilen MJ, Heuts PHTG, Crombez G. Graduele exposure in vivo bij pijngerelateerde angst. Ned Tijdschr Fysiotherapie 2002: 112; 50-57.

De Jong JR, Vlaeyen JWS, Geilen MJ. Graduele exposure in vivo bij pijngerelateerde angst. Psychoskoop 2001: 12; 7-24.

De Jong JR, Vlaeyen JWS, Kerkhoffs-Hanssen M. Graduele exposure in vivo en gedrasgsexperimenten bij pijngerelateerde vrees. Mattie et al. (Red) Pijninformatorium 2001: PT1500; 1-13. 
De Jong JR, Vlaeyen JWS, Geilen MJ, Heuts PHTG. De angst voor bewegen: geleidelijke exposure in vivo bij chronische lage rugpijn. Dth, Tijdschrift voor Directieve Therapie 2000: 20; 143-161.

Goossens M, De Jong JR. Verslag van een belangwekkend BPS symposium over fibromyalgie: "new disease of social illness". Nederlands Tijdschrift voor Pijn en Pijnbestrijding 1999: 19 (2); 42.

\section{Cat.: Book Contributions}

Vlaeyen JWS, de Jong J, Crombez G.Graduele exposure in vivo. In: Vlaeyen JWS, Heuts PHTG (Red.), Gedragsgeoriënteerde behandelingsstrategieën bij rugpijn. Cure \& Care development. Houten/Diegem Bohn, Satfleu, Van Loghum, 2000. ISBN 90 31332097.

Vlaeyen JWS, De Jong JR, Heuts PHTG, Crombez G. Graded exposure in vivo for pain-related fear. The case of chronic musculoskeletal pain. In: Rice A, Warfield C, Justins D, Eccleston C, eds. Handbook of Clinical Pain Management. Practical applications and procedures. London: Arnold, 2002. ISBN 0340731540.

Johan WS Vlaeyen, Jeroen R de Jong, Judith Sieben, Geert Crombez. Graded Exposure In Vivo for Pain-Related Fear. In: Dennis C Turk, Robert J Gatchel (Ed.), Psychological Approaches to Pain Management, A Practitioner's Handbook - 2nd ed. New york, The Guilford Press, 2002. ISBN 1572306424.

Vlaeyen JWS, de Jong JR, Leeuw M, Crombez G. Fear-reduction through exposure in vivo. In: Asmundson G, Vlaeyen JWS, Crombez G. Understanding and treating fear in pain. Oxford; Oxford University Press, 2004. ISBN 0198525141.

Jeroen de Jong, Johan Vlaeyen, Corine Cuijpers, Mario Geilen. De toepassing en complicaties van graduele exposure in vivo met gedragsexperimenten bij pijngerelateerde vrees. In: Dijkstra PU, et al. (Red.), Jaarboek Fysiotherapie Kinesitherapie. Bohn Stafleu Van Loghum, Houten, 2005. ISBN 9031343110.

Jeroen R de Jong, Johan WS Vlaeyen. (2003). Graded Exposure In Vivo and Behavioral Experiments for Pain-Related Fear. In: Robert F. Schmidt, William D. Willis (Ed.), Encyclopedic Reference of Pain. (submitted)

Patrick Onghena, Johan WS Vlaeyen, Jeroen R de Jong (2005). Randomized replicated single-case experiments: Treatment of pain-related fear by graded exposure 
in vivo. Submitted for publication in S Sawilowsky (Ed.), Real data analysis. American Research Association.

\section{Articles submitted for publication}

\section{Cat.: International SCl or SSCl indexed.}

Jeroen R. de Jong, Johan W.S. Vlaeyen, Jantha M. de Gelder, Jaap Patijn. Painrelated fear, perceived harmfulness of activities and functional limitations in Complex Regional Pain Syndrome type I.

Jeroen R de Jong, Marlies den Hollander, Isis Bulté, Joop Ruijgrok, Johan WS Vlaeyen. Generalization of Graded Exposure in Vivo in Complex Regional Pain Syndrome type I (CRPS-I).

Jeroen R. de Jong, Johan W.S. Vlaeyen, Christoph Loo, Marjon van Eijsden, Joop Ruijgrok. Reduction of pain-related fear in work-related upper extremity pain (WRUEP): a rationale for graded exposure in vivo. 